\title{
Princípio de reconhecimento de espaços de laços relativos
}

\author{
Renato Vasconcellos Vieira
}

TESE APRESENTADA

AO

Instituto de Matemática e Estatística

DA

Universidade de São Paulo

PARA

OBTENÇÃO DO TÍTULO

$\mathrm{DE}$

Doutor em Ciências

\author{
Programa: Matemática \\ Orientador: Prof. Dr. Daciberg Lima Gonçalves \\ Coorientador: Prof. Dr. Eduardo Outeiral Correa Hoefel
}





\section{Princípio de reconhecimento de espaços de laços relativos}

Esta versão da tese contém as correções e alterações sugeridas pela Comissão Julgadora durante a defesa da versão original do trabalho, realizada em 15/06/2018. Uma cópia da versão original está disponível no Instituto de Matemática e Estatística da Universidade de São Paulo.

Comissão Julgadora:

- Prof. Dr. Daciberg Lima Goncalves (orientador) - IME-USP

- Prof. Dr. Eduardo Outeiral Correa Hoefel (coorientador) - UFPR

- Prof. Dr. Oziride Manzoli Neto - ICMC-USP

- Prof. Dr. Tomas Edson Barros - UFSCar

- Prof. Dr. Marcos Benevenuto Jardim - UNICAMP 

Play your ukulele

Amanda F. Palmer

The unknown thing to be known appeared to me as some stretch of earth or hard marl, resisting penetration... The sea advances insensibly in silence, nothing seems to happen, nothing moves, the water is so far off you hardly hear it... Yet it finally surrounds the resistant substance

Alexander Grothendieck 



\section{Agradecimentos}

À CAPES e CNPQ, pelo financiamento que tornou essa pesquisa possível.

Ao Daciberg Gonçalves, pelos anos de orientação, cuidado e atenção. Por prestar atenção nos meus interesses e nutrir minha curiosidade. Por entender minhas dificuldades e me apontar na direção certa.

Ao Eduardo Hoefel, por incentivar meus devaneios e manter meus pés no chão.

Às professoras e professores do IME-USP por tudo que me ensinaram.

Às funcionárias e funcionários do IME-USP cujo esforço garantem um ambiente em que essa pesquisa foi possível, em particular às secretarias do departamento de matemática que me salvaram de mim mesmo mais vezes do que consigo contar.

À Luiza Vasconcellos, por sempre nutrir minha curiosidade e acreditar em mim. Pelo amor e amizade com o qual sempre pude contar.

Ao Elias Vieira, por todo o apoio e amor que sempre demonstrou.

À Erica Martinelli, por ser um raio de luz que ilumina a beleza do mundo. Pelo companheirismo na nossa viagem pelo tempo-espaço. Por compartilhar comigo a sua poesia. Pela amizade e o amor profundo que dividimos. Por me abrir.

Às miga tudo, por trazer mais cor e música à minha vida. 



\section{Resumo}

VIEIRA, R. V. Princípio de reconhecimento de espaços de laços relativos. Tese (Doutorado) Instituto de Matemática e Estatística, Universidade de São Paulo, São Paulo, 2018.

O princípio de reconhecimento de espaços de $\infty$-laços é que o funtor $\Omega^{\infty}: \mathrm{Sp} \rightarrow \mathcal{E}^{\infty}$ [Top] dado por $\Omega^{\infty} Y_{\bullet}=$ colim ${ }_{\bullet \rightarrow \infty} \Omega^{\bullet} Y_{\bullet}$ induz uma equivalência entre a categoria homotópica de espectros conectivos e a categoria homotópica de $\mathcal{E}^{\infty}$-álgebras grouplike para qualquer resolução cofibrante $\mathcal{E}^{\infty}$ do operad Com de monóides comutativos. Nesta tese é provado um princípio de reconhecimento de 2-espaços de $N$-laços para $2<N \leq \infty$. Quando $N=\infty$ esse princípio afirma

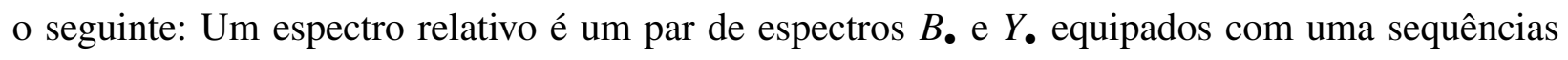
de aplicações pontuadas $\iota_{\bullet}: B_{\bullet} \rightarrow Y_{\bullet+1}$ compatíveis com as estruturas de espectros. Um espectro relativo é conectivo se o par de espectros subjacentes forem conectivos. Denotamos a categoria de espectros relativos por $\mathrm{Sp}^{\nearrow}$ e de espectros relativos conectivos por $\mathrm{Sp}_{0}^{\nearrow}$. Um $2 E_{\infty}$-operad é uma resolução cofibrante $\mathcal{E}_{2}^{\infty}$ do 2-operad $\mathrm{Com}^{\rightarrow}$ de homomorfismos de monóides comutativos. Uma $\mathcal{E}_{2}^{\infty}$-álgebra $\left(X_{c}, X_{o}\right)$ é grouplike se $X_{c}$ e $X_{o}$ forem grouplike. Denotamos a categoria de $\mathcal{E}_{2}^{\infty}$ álgebras por $\mathcal{E}_{2}^{\infty}$ [Top] e a categoria de $\mathcal{E}_{2}^{\infty}$-álgebras grouplike por $\mathcal{E}_{2}^{\infty}$ [Top $]_{G r p}$. O 2-espaço de $\infty$-laços de um espectro relativo é o par de espaços $\Omega_{2}^{\infty} \iota_{\bullet}:=\operatorname{colim} \cos _{\bullet}\left(\Omega^{\bullet} Y_{\bullet}, \Omega_{\text {rel }}^{\bullet} \iota_{\bullet}\right)$. Temos que as imagens do funtor $\Omega_{2}^{\infty}$ admitem uma estrutura natural de $\mathcal{E}_{2}^{\infty}$-álgebra, logo $\Omega_{2}^{\infty}$ define um funtor $\mathrm{Sp}^{\nearrow} \rightarrow \mathcal{E}_{2}^{\infty}$ [Top]. Existe um funtor $B_{2}^{\infty}: \mathcal{E}_{2}^{\infty}[\mathrm{Top}] \rightarrow \mathrm{Sp}^{\nearrow}$ e uma adjunção ( $\mathbb{L} B_{2}^{\infty} \dashv \mathbb{R} \Omega_{2}^{\infty}$ ) entre as categorias homotópicas $\mathcal{H}_{o} \mathcal{E}_{2}^{\infty}$ [Top] e $\mathcal{H} o \mathrm{Sp}^{\nearrow}$ que induzem uma equivalência entre as categorias homotópicas $\mathcal{H}_{o \mathcal{E}_{2}^{\infty}}[\mathrm{Top}]_{G r p}$ e $\mathcal{H} o \mathrm{Sp}_{0}^{\nearrow}$.

Palavras-chave: Operads, 2-operads, espectros, espectros relativos, espaços de laços, espaços de laços relativos, princípio de reconhecimento, princípio de reconhecimento relativo. 



\section{Abstract}

VIEIRA, R. V. Recognition principle of relative loop spaces. Thesis ( $\mathrm{PhD})$ - Instituto de Matemática e Estatística, Universidade de São Paulo, São Paulo, 2018.

The recognition principle of $\infty$-loop spaces is that the functor $\Omega^{\infty}: \mathrm{Sp} \rightarrow \mathcal{E}^{\infty}$ [Top] defined by $\Omega^{\infty} Y_{\bullet}=$ colim $_{\bullet \rightarrow \infty} \Omega^{\bullet} Y_{\bullet}$ induces an equivalence between the homotopy category of connective spectra and the homotopy category of grouplike $\mathcal{E}^{\infty}$-algebras for any cofibrant resolution $\mathcal{E}^{\infty}$ of the commutative monoid operad Com. In this thesis a relative recognition principle of $N$-loop 2-spaces is proved for $2<N \leq \infty$. For $N=\infty$ this principle states the following: A relative spectrum is a pair of spectra $B_{\bullet}$ and $Y_{\bullet}$ equipped with a sequence of pointed maps $\iota_{\bullet}: B_{\bullet} \rightarrow Y_{\bullet+1}$ compatible with the spectrum structures. A relative spectrum is connective if the underlying pair of spectra are connective. The category of relative spectra is denoted by $\mathrm{Sp}^{\prime}$ and the category of connective relative spectra by $\mathrm{Sp}_{0}^{\nearrow}$. A $2 E_{\infty}$-operad is a cofibrant resolution $\mathcal{E}_{2}^{\infty}$ of the commutative monoid homomorphism 2-operad $\mathrm{Com}^{\rightarrow}$. An $\mathcal{E}_{2}^{\infty}$-algebra $\left(X_{c}, X_{o}\right)$ is grouplike if $X_{c}$ and $X_{o}$ are grouplike. The category of $\mathcal{E}_{2}^{\infty}$-algebras is denoted by $\mathcal{E}_{2}^{\infty}[\mathrm{Top}]$ and the category of grouplike $\mathcal{E}_{2}^{\infty}$ algebras by $\mathcal{E}_{2}^{\infty}[\mathrm{Top}]_{G r p}$. The $\infty$-loop 2-space of a relative spectrum is the pair of pointed spaces $\Omega_{2}^{\infty} \iota_{\bullet}:=\operatorname{colim}_{\bullet \rightarrow \infty}\left(\Omega^{\bullet} Y_{\bullet}, \Omega_{\text {rel }}^{\bullet} \iota_{\bullet}\right)$. The images of the functor $\Omega_{2}^{\infty}$ admit an $\mathcal{E}_{2}^{\infty}$-algebra structure, therefore $\Omega_{2}^{\infty}$ defines a functor $\mathrm{Sp}^{\nearrow} \rightarrow \mathcal{E}_{2}^{\infty}$ [Top]. The infinite relative recognition principle is that there is a functor $B_{2}^{\infty}: \mathcal{E}_{2}^{\infty}[\mathrm{Top}] \rightarrow \mathrm{Sp}^{\top}$ and a derived adjunction $\left(\mathbb{L} B_{2}^{\infty} \dashv \mathbb{R} \Omega_{2}^{\infty}\right)$ between the homotopy categories $\mathcal{H} o \mathcal{E}_{2}^{\infty}[\mathrm{Top}]$ and $\mathcal{H} o \mathrm{Sp}^{\top}$ that induce an equivalence beteween the homotopy categories $\mathcal{H} o \mathcal{E}_{2}^{\infty}[\mathrm{Top}]_{\text {Grp }}$ and $\mathcal{H} o \mathrm{Sp}_{0}^{\nearrow}$.

Palavras-chave: Operads, 2-operads, spectra, relative spectra, loop spaces, relative loop spaces, recognition principle, relative recognition principle. 



\section{Sumário}

Sumário $\quad$ viii

$\begin{array}{ll}\text { Lista de Símbolos } & \text { ix }\end{array}$

$\begin{array}{ll}\text { Introdução } & 1\end{array}$

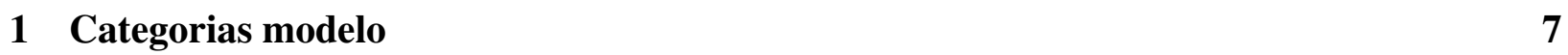

1.1 Estrutura modelo . . . . . . . . . . . . . . . . . . . . . . . . 9

1.2 Homotopia e categoria homotópica . . . . . . . . . . . . . . . . . 15

1.3 Quasi-adjunções fracas de Quillen . . . . . . . . . . . . . . . . . . . 22

1.4 Categorias modelo cofibrantemente geradas e transferência de estrutura modelo . . 26

1.5 Categorias pontuadas . . . . . . . . . . . . . . . 31

1.6 Categorias de Reedy generalizadas . . . . . . . . . . . . . . . . . . . 32

1.7 Categorias modelo próprias . . . . . . . . . . . . . . . . 37

1.8 Localização de Bousfield . . . . . . . . . . . . . . . . . . . . . . . . . 39

1.9 Quasi-adjunções idempotentes de Quillen . . . . . . . . . . . . . . . . 46

2 Categorias monoidais fechadas $\quad 47$

2.1 Estrutura monoidal . . . . . . . . . . . . . . . . . . . . 48

2.2 Categorias modelo monoidais . . . . . . . . . . . . . . . . 52

2.3 Monadas e álgebras . . . . . . . . . . . . . . . . . . . . 55

3 Espaços topológicos, espaços de laços e espectros

3.1 Espaços compactamente gerados fracamente Hausdorff . . . . . . . . . . . . . . 60

3.2 Espaços de laços e espectros . . . . . . . . . . . . . . . . 63 
viii SUMÁRIO

3.3 2-Espaços de laços relativos e espectros relativos . . . . . . . . . . . . 67

3.4 Realização geométrica . . . . . . . . . . . . . . . . . . 70

4 Operads e 2-operads $\quad 73$

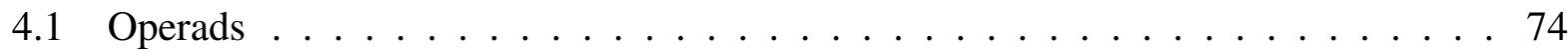

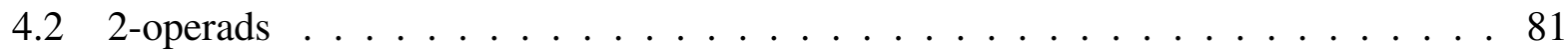

4.3 Estrutura modelo em 2-operads e álgebras sobre 2-operads . . . . . . . . . . . . . 87

4.4 Resoluções de Boardman-Vogt . . . . . . . . . . . . . . . . . . . . . . 91

5 Princípio de reconhecimento de espaços de laços relativos 97

5.1 Teorema de aproximação . . . . . . . . . . . . . . . . . . . . . 98

5.2 Compatibilidade da realização geométrica . . . . . . . . . . . . . . . . . . 104

5.3 Principio de reconhecimento de espaços de laços finitos . . . . . . . . . . . . . 106

5.4 Princípio de reconhecimento de laços relativos infinitos . . . . . . . . . . . . . 110

A (Não-)formalidade do 2-operad de queijos suíços de Voronov em dimensão 2

Referências Bibliográficas 


\section{Lista de Símbolos}

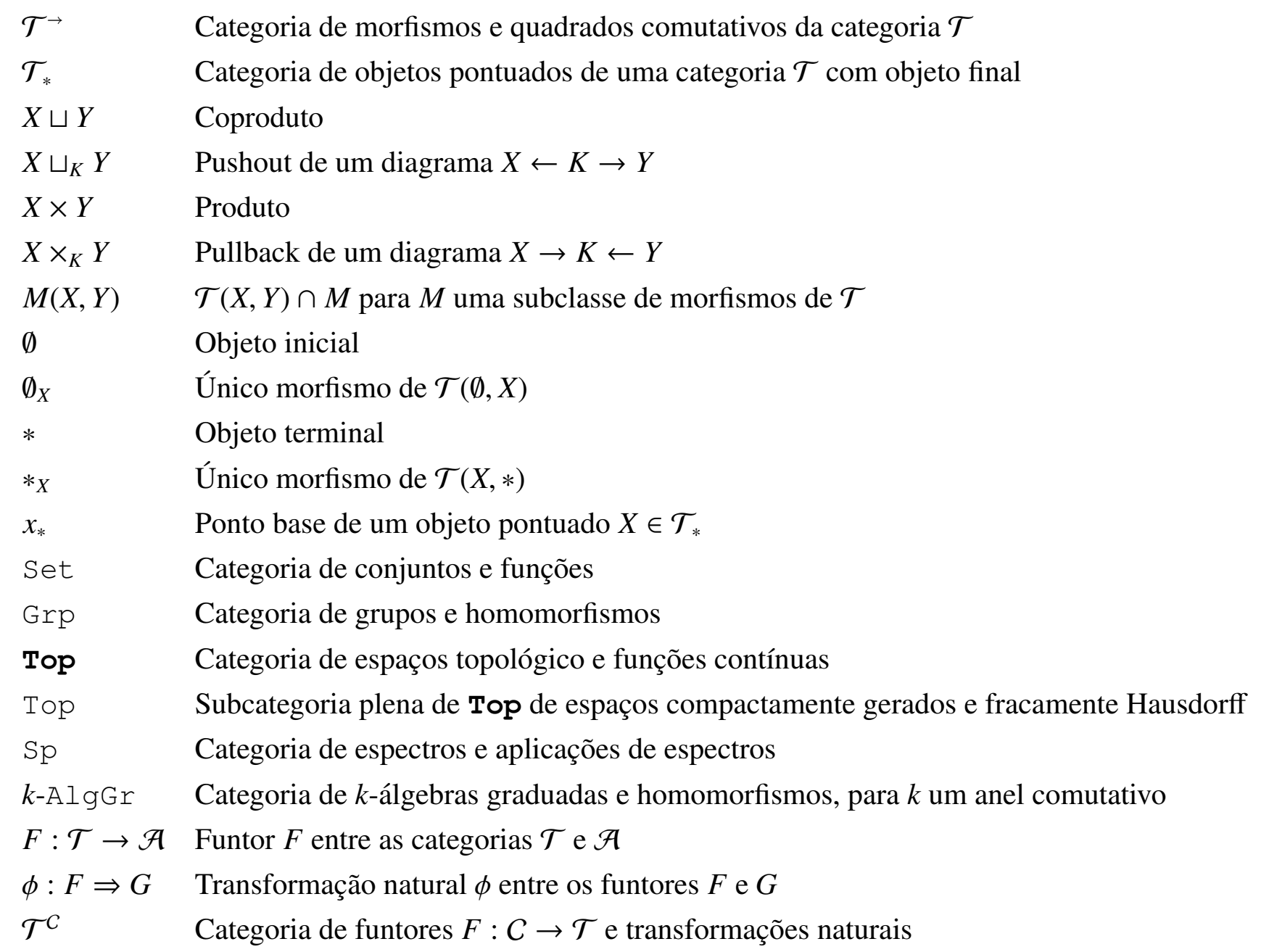





\section{Introdução}

O princípio de reconhecimento de espaços de laços infinitos é que o funtor derivado do funtor de espaço de laços infinito nos dá uma equivalência entre a categoria homotópica de espectros conectivos e a categoria homotópica de $\mathcal{E}^{\infty}$-espaços grouplike [Ma72, Ma74].

Um espectro é uma sequência de espaços pontuados $Y_{\bullet} \in \prod_{\bullet \in \mathbb{N}} \mathrm{T}_{0} \mathrm{p}_{*}$, equipada com uma sequência de aplicações estruturais $\sigma_{\bullet} \in \prod_{\bullet \in \mathbb{N}} \mathrm{T}_{0}\left(\Sigma^{1} Y_{\bullet}, Y_{\bullet+1}\right)$ [Li58]. Denotamos a categoria de espectros por Sp. Espectros são objetos centrais da topologia algébrica pois toda teoria de (co)homologia generalizada é determinada por um espectro [Sw75]. Note que pela adjunção $\left(\Sigma^{1} \dashv\right.$ $\left.\Omega^{1}\right)$ também temos uma sequência de aplicações estruturais duais $\tilde{\sigma}_{\bullet} \in \prod_{\bullet \in \mathbb{N}} \operatorname{Top}_{*}\left(Y_{\bullet}, \Omega^{1} Y_{\bullet+1}\right)$, que podemos usar para definir o espaço de laços infinitos do espectro $\Omega^{\infty} Y_{\bullet}:=\operatorname{colim} \boldsymbol{\bullet}_{\rightarrow \infty} \Omega^{\bullet} Y_{\bullet}$. Pela naturalidade dessa construção temos um funtor $\Omega^{\infty}: \mathrm{Sp} \rightarrow \mathrm{Top}_{*}$. Os grupos de homotopia estáveis de um espectro é a sequência de grupos abelianos $\pi_{q}^{S} Y_{\bullet}:=\operatorname{colim} \bullet_{\bullet \rightarrow \infty} \pi_{\bullet+q} Y_{\bullet} \in \prod_{q \in \mathbb{Z}} \mathrm{Grp} \mathrm{Pb}_{\mathrm{Ab}}$. Dizemos que um espectro $Y_{\bullet}$ é conectivo se $\pi_{q}^{S} Y_{\bullet}$ é trivial para $q<0$.

Podemos equipar a categoria de espaços topológicos Top com a estrutura modelo de Quillen [Hi15], cujas equivalências fracas são as equivalências homotópicas fracas, i.e., as aplicações que induzem isomorfismos nos conjuntos de componentes conexos e nos grupos de homotopia, e os objetos bifibrantes são retrações de CW-complexos. Essa estrutura modelo induz uma estrutura modelo na categoria de espaços pontuados $\mathrm{Top}_{*}$ e também uma em $\mathrm{Sp}$, a estrutura modelo estrita, que admite uma localização de Bousfield à esquerda, i.e., uma estrutura modelo com as mesmas cofibrações e com mais equivalências fracas, cujas equivalências fracas são as equivalências homotópicas fracas estáveis, i.e., os morfismos que induzem isomorfismos nos grupos de homotopia estáveis, e cujos objetos bifibrantes são retrações de CW-espectros, i.e., espectros compostos por retrações de $\mathrm{CW}$-complexos cujas aplicações estruturais $\sigma$. são cofibrações, que também são $\Omega$-espectros, i.e., espectros tais que as aplicações estruturais duais $\tilde{\sigma}_{\bullet}$ são equivalências fracas [BF78, Sc97]. O funtor $\Omega^{\infty}$ é um adjunto de Quillen à direita, i.e., ele admite um adjunto à esquerda $\Sigma^{\infty}: \mathrm{Top}_{*} \rightarrow$ Sp e preserva fibrações e fibrações triviais. Isso implica a existência de uma adjunção derivada entre as categorias homotópicas $\left(\mathbb{L} \Sigma^{\infty} \dashv \mathbb{R} \Omega^{\infty}\right): \mathcal{H} o \mathrm{Top}_{*} \rightleftharpoons \mathcal{H} o \mathrm{Sp}$.

As imagens de $\Omega^{\infty}$ admitem estruturas de álgebras naturais sobre $E_{\infty}$-operads. Um operad topológico $\mathcal{P}$ é uma sequência de $\mathbb{S}_{\underline{n}}$-espaços $\mathcal{P}(\underline{n}) \in \prod_{n \in \mathbb{N}}$ Top $\mathbb{S}_{\underline{n}}$ tais que $\mathcal{P}(\underline{0})=*$, com $\mathbb{S}_{\underline{n}}$ o grupo de simetrias de $n$ elementos, equipados com morfismos estruturais que induzem uma estrutura de monada no funtor $P: \mathrm{Top}_{*} \rightarrow$ Top $\mathrm{p}_{*}$ definido pelo coend $P(X):=\int^{\mathbb{S}_{\mathrm{inj}}} \mathcal{P}(\underline{n}) \times X^{n}$ 
[Ma72]. Denotamos a categoria de álgebras sobre a monada associada a um operad por $\mathcal{P}$ [Top]. Essa categoria também herda uma estrutura modelo da estrutura modelo de Quillen nos espaços topológicos [BM03]. Para cada $N \in \mathbb{N}$ temos o operad dos pequenos $N$-cubos $C^{N}$ tal que $C^{N}(\underline{n})$ é o subespaço de $\left[\coprod_{n} I^{N}, I^{N}\right]$ das aplicações definidas por translações e dilatações dos $N$-cubos cujas imagens dos interiores são disjuntos. Esses operads admitem uma sequência de aplicações naturais $\kappa_{\bullet} \in \prod_{\bullet \in \mathbb{N}} \operatorname{Top}\left(C^{\bullet}, C^{\bullet+1}\right)$ e definimos $C^{\infty}:=\operatorname{colim}{ }_{\bullet \rightarrow \infty} C^{\bullet}$. O operad $C^{\infty}$ tem a propriedade que os espaços $C^{\infty}(\underline{n})$ são contrateis. As imagens do funtor $\Omega^{\infty}$ admitem uma estrutura natural de $C^{\infty}$-álgebra dadas por uma transformação natural $\theta^{\infty}: C^{\infty} \Omega^{\infty} \Rightarrow \Omega^{\infty}$ [BV68]. A categoria de operads topológicos também herda uma estrutura modelo da estrutura modelo de Quillen de espaços topológicos [BM03]. Dizemos que um operad $\mathcal{E}^{\infty}$ é um $E_{\infty}$-operad se ele for cofibrante e os espaços $\mathcal{E}^{\infty}(\underline{n})$ são contrateis. Equivalentemente um $E_{\infty}$-operad é uma resolução cofibrante do operad $C o m$ cujas álgebras são monoides topológicos comutativos. Para todo $E_{\infty}$-operad $\mathcal{E}^{\infty}$ temos uma equivalência fraca de operads $\pi: \mathcal{E}^{\infty} \Rightarrow C^{\infty}$ que induz uma estrutura de $\mathcal{E}^{\infty}$-álgebras nas imagens de $\Omega^{\infty}$. Temos então que $\Omega^{\infty}$ induz um funtor $\left(\Omega^{\infty}, \theta^{\infty}\right): \mathrm{Sp} \rightarrow \mathcal{E}^{\infty}$ [Top] que preserva fibrações e fibrações triviais, e portanto admite derivação à direita.

Esse funtor não admite um adjunto à esquerda, porém podemos usar a estrutura de $C^{\infty}$-álgebra dos espaços de laços infinitos e a construção bar introduzida por May em [Ma72] para definir um funtor que induz uma adjunção das categorias homotópicas. Temos o morfismo de monadas em espaços topológicos pontuados $\alpha^{\infty}:=\theta_{\Sigma^{\infty}}^{\infty} C^{\infty} \eta^{\infty}: C^{\infty} \Rightarrow \Omega^{\infty} \Sigma^{\infty}$. Em [Ma74] May provou o teorema de aproximação que diz que $\alpha_{X}^{\infty}$ é um completamento de grupo para todo espaço pontuado $X$, i.e., $\alpha_{X}^{\infty}$ induz um isomorfismo da $k$-álgebra graduada $H_{\bullet}\left(C^{\infty} X, k\right)\left[\pi_{0}^{-1}\right]$, a localização do anel $H_{\bullet}\left(C^{\infty} X, k\right)$ no subanel de componentes conexas, em $H_{\bullet}\left(\Omega^{\infty} \Sigma^{\infty} X, k\right)$ para todo anel comutativo de coeficientes $k$. Se $X$ é uma $\mathcal{E}^{\infty}$-álgebra grouplike, i.e., o monoide $\pi_{0} X$ é um grupo, então o teorema dual de Whitehead nos dá que $\alpha_{X}^{\infty}$ é uma equivalência fraca. Para uma monada $C$, uma $C$-álgebra $X$ e um $C$-funtor $F$ em Top a construção bar nos dá um espaço pontuado simplicial com $B^{\langle\bullet\rangle}(F, C, X):=F C^{\bullet} X$. Definimos $B(F, C, X)$ como a realização geométrica desse espaço simplicial. Podemos então definir um funtor $B^{\infty}: \mathcal{E}^{\infty}[\mathrm{Top}] \rightarrow \operatorname{Sp} \operatorname{como} B^{\infty} X_{\bullet}:=B\left(\Sigma^{\bullet}, \overline{C^{\bullet}}, X\right)$, onde $\overline{C^{\bullet}}$ são monadas associadas à resoluções cofibrantes $\overline{C^{\bullet}}$ dos operads dos pequenos cubos $C^{\bullet}$. Esse funtor preserva objetos cofibrantes e equivalências fracas entre eles, e portanto admite uma derivada à esquerda. No geral $\mathcal{E}^{\infty}[\mathrm{Top}]\left(X, \Omega^{\infty} B^{\infty} X\right)$ pode ser vazio (apesar de existir uma aplicação de espaços topológicos induzido da unidade da adjunção $\left(\Sigma^{\infty} \dashv \Omega^{\infty}\right)$ ), porém temos um span natural

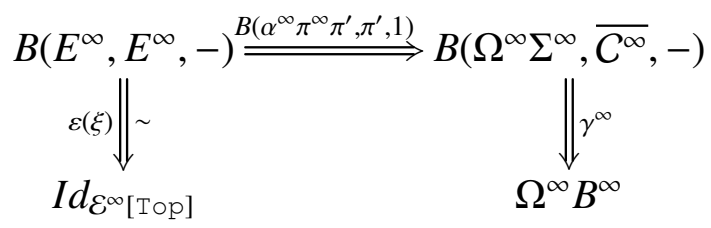

e também uma transformação natural $\varepsilon\left(\epsilon^{\infty}\right): B^{\infty} \Omega^{\infty} \Rightarrow I d_{\mathrm{Sp}}$. 
Essas estruturas induzem uma adjunção das categorias homotópicas

$$
\left(\mathbb{L} B^{\infty} \dashv \mathbb{R} \Omega^{\infty}\right): \mathcal{H} o \mathcal{E}^{\infty}[\mathrm{Top}] \rightleftharpoons \mathcal{H} o \mathrm{Sp} .
$$

Os funtores $\Omega^{\infty}$ e $B^{\infty}$ definem uma localização de Bousfield à esquerda de $\mathcal{E}^{\infty}$ [Top] cujas equivalências fracas são as $\mathcal{E}^{\infty}$-aplicações $f \in \mathcal{E}^{\infty}$ [Top] $\left(X, X^{\prime}\right)$ que induzem homomorfismos $\bar{f}_{*} \in$ $k-\operatorname{AlgGr}\left(H(X, k)\left[\pi_{0}^{-1}\right], H\left(X^{\prime}, k\right)\left[\pi_{0}^{-1}\right]\right)$ que são isomorfismos para todo anel de coeficientes $k \mathrm{e}$ cujos objetos fibrantes são os $\mathcal{E}^{\infty}$-espaços grouplike, e também uma localização de Bousfield à direita de $\mathrm{Sp}$ cujas equivalências fracas são as aplicações de espectros $g_{\bullet} \in \operatorname{Sp}\left(Y_{\bullet}, Y_{\bullet}^{\prime}\right)$ tais que $\pi_{q}^{S} g \in \operatorname{Grp}_{\mathrm{Ab}}\left(\pi_{q}^{S} Y_{\bullet}, \pi_{q}^{S} Y_{\bullet}^{\prime}\right)$ é um isomorfismo para todo $q \geq 0$ e cujos objetos cofibrantes são os espectros conectivos. Temos então que a subcategoria plena $\mathcal{H} o \mathcal{E}^{\infty}$ [Top $]_{\text {Grp }}$ de $\mathcal{H} o \mathcal{E}^{\infty}$ [Top] composta pelas $\mathcal{E}^{\infty}$-álgebras grouplike é uma subcategoria reflexiva, e a subcategoria $\mathcal{H} o \mathrm{Sp}_{0}$ de $\mathcal{H} o \mathrm{Sp}$ composta pelos espectros conectivos é uma subcategoria correflexiva, e temos que a adjunção $\left(\mathbb{L} B^{\infty} \dashv \mathbb{R} \Omega^{\infty}\right.$ ) é uma equivalência quando restrita nessas subcategorias. Isso nos dá o princípio de reconhecimento para espaços de laços infinitos. Esse resultado foi estendido para uma equivalência entre espaços pontuados $N$-1-conexos e $\overline{C^{N}}$-álgebras grouplike para qualquer resolução cofibrante $\overline{C^{N}}$ de $C^{N}$ usando resultados de aproximação de Cohen [CLM76, III.3.3] para $1<N<\infty$, e por um argumento distinto de Stasheff no caso $N=1$ [StJ63]. O caso $N=1$ precisa de um argumento distinto porque os resultados de aproximação para $N>1$ usam o fato que $C^{N}$-álgebras são comutativas a menos de homotopia, o que não é o caso para $C^{1}$-álgebras.

Nesta tese uma versão relativa do princípio de reconhecimento é provado. Um espectro relativo é um par de espectros $B_{\text {• e }} Y_{\bullet}$ equipados com uma sequências de aplicações pontuadas $\iota_{\bullet} \in \prod_{\bullet \in \mathbb{N}} \operatorname{Top}\left(B_{\bullet}, Y_{\bullet+1}\right)$ compativel com as estruturas de espectros. Denotamos um espectro relativo simplesmente por $\iota_{\text {. }}$ O 2-espaço de laços infinitos de um espectro relativo é o par de espaços $\Omega_{2}^{\infty} \iota_{\bullet}:=$ colim ${ }_{\bullet \rightarrow \infty}\left(\Omega^{\bullet} Y_{\bullet}, \Omega_{\text {rel }}^{\bullet} \iota_{\bullet}\right)$. Temos que as imagens do funtor $\Omega_{2}^{\infty}$ admitem uma estrutura de álgebras sobre qualquer $2 E_{\infty}$-operad $\mathcal{E}_{2}^{\infty}$, que são operads coloridos em duas cores ordenadas cofibrantes cujos espaços subjacentes são todos contráteis. Usando construções análogas ao caso absoluto neste trabalho é provado que $\Omega_{2}^{\infty}$ induz uma equivalência entre espectros relativos conectivos e $\mathcal{E}_{2}^{\infty}$-álgebras grouplike via um funtor de espectro relativo de classificação $B_{2}^{\infty}$. Os $2 E_{\infty}$-operads são resoluções cofibrantes do 2-operad $\mathrm{Com}^{\rightarrow}$ cujas álgebras são homomorfismos de monóides topológicos comutativos. A demonstração segue de uma versão relativa do teorema de aproximação, que por sua vez segue da construção de uma quasi-fibração $p_{o}^{\infty}: \pi_{o} S C^{N}\left(X_{c}, X_{o}\right) \rightarrow C^{N-1} X_{o}$ para cada $N \in \mathbb{N} \cup\{\infty\}$, aonde $S C^{N}$ é a monada associada ao 2-operad dos queijos-suíço.

Teorema. Os funtores $B_{2}^{\infty}$ e $\Omega_{2}^{\infty}$ induzem uma equivalência de categorias homotópicas:

$$
\left(\mathbb{L} B_{2}^{\infty} \dashv \mathbb{R} \Omega_{2}^{\infty}\right): \mathcal{H} o \mathcal{E}_{2}^{\infty}[T o p]_{\text {Grp }} \rightleftharpoons \mathcal{H} o S p_{\text {Con }}^{\top}
$$

Os teoremas de aproximação de Cohen [CLM76, III.3.3] nos permitem estender esse resultado para $2<N<\infty$. 
Teorema. Seja $2<N<\infty$. Os funtores $B_{2}^{N}$ e $\Omega_{2}^{N}$ induzem uma equivalência de categorias homotópicas:

$$
\left(\mathbb{L} B_{2}^{N} \dashv \mathbb{R} \Omega_{2}^{N}\right): \mathcal{H} o \overline{S C^{N}}[\text { Top }]_{\text {Grp }} \rightleftharpoons \mathcal{H o T o p} \vec{N}-1
$$

O caso $N=1$ pode ser derivado do resultado de reconhecimento relativo de Hoefel, Livernet e Stasheff em [HLS16]. A demonstração na presente tese funciona para os casos $N=1,2$ restritos às $C^{N}$-álgebras conexas e espaços relativos $N$-conexos, porém o caso geral para $C^{2}$-álgebras grouplike arbitrárias continua aberto.

Este trabalho está organizado da seguinte maneira. No primeiro capítulo apresentamos a teoria de categorias modelo que usamos para descrever as estruturas homotópicas das categorias do teorema de reconhecimento relativo. A maior parte dos resultados desse capítulo podem ser encontrados nas referências padrões de teoria de categorias modelo como [GJ09, Hi09, HoM07]. Descreveremos as principais propriedades de categorias modelo e como construir suas categorias homotópicas. Introduzimos quasi-adjunções fracas de Quillen, que nos dão condições mais fracas que adjunções de Quillen, ou mesmo adjunções de Quillen fracas, para que pares de funtores induzam adjunções nas categorias homotópicas. Precisamos dessa generalização pois o princípio de reconhecimento nos dá apenas um span entre uma $S C^{N}$-álgebra $\left(X_{o}, X_{c}\right)$ e $\Omega_{2}^{N} B_{2}^{N}\left(X_{o}, X_{c}\right)$ que induz a unidade da adjunção na categoria homotópica. A definição explícita dessa generalização parece ser nova, porém estava implícita no resultado de reconhecimento de May em [Ma72]. Descreveremos também como obter uma estrutura modelo por transferência via adjunção e por localizações de Bousfield. Introduzimos uma generalização do método de localização de Bousfield à esquerda via monadas idempotentes de [BF78] que nos permite fazer localizações usando certas quasiadjunções de Quillen fracas que induzem o que chamamos de quasi-monadas idempotentes. Temos um dual desse resultado que nos dá localizações de Bousfield à direita via quasi-comonadas idempotentes. Nesse contexto podemos descrever o princípio de reconhecimento relativo infinito como uma equivalência associada a uma quasi-adjunção idempotente de Quillen fraca.

No segundo capítulo descrevemos a teoria de categorias modelo monoidais [HoM07], que é a estrutura em uma categoria que nos permite construir categorias modelo de álgebras sobre operads quando a categoria admite um objeto intervalo de Hopf cocomutativo, que é uma generalização do intervalo $I=[0,1]$ em Top [BM03, BM07]. Também apresentamos a construção bar que é uma componente central do princípio de reconhecimento.

No terceiro capítulo apresentamos a categoria monoidal fechada Top dos espaços compactamente gerados e fracamente Hausdorff [StN09] e descrevemos a estrutura modelo de Quillen nela[Hi15]. Descrevemos também a categoria modelo induzida de espaços relativos e a estrutura modelo estável de espectros[BF78, Sc97] e a de espectros relativos. Recordamos o fato clássico que o funtor de espaços de laços $\Omega^{N}$ admite um adjunto de Quillen à esquerda $\Sigma^{N}$ e mostramos que $\Omega_{2}^{N}$ admite um adjunto de Quillen à esquerda fraco $\Sigma_{2}^{N}$. Mostramos que para $N<\infty$ a adjunção $\left(\Sigma_{2}^{N} \dashv \Omega_{2}^{N}\right)$ induz uma estrutura modelo na categoria de aplicações pontuadas cujos objetos cofibrantes são retrações de CW-pares $N-1$-conexos. Terminamos o capítulo apresentando a 
construção de realização geométrica de espaços simpliciais como um coend.

No quarto capítulo descrevemos a teoria de operads e de operads coloridos em duas cores ordenadas $\{c<o\}$, que chamamos de 2-operads. Nos focamos nos operads dos pequenos $N$ cubos $C^{N}$ de Boardman e Vogt [BV68] e nos 2-operads dos $N$-queijos suíços $S C^{N}$ do Voronov [Vo99]. O 2-operad dos 2-queijos suíços $S C^{2}$ foi introduzido por Voronov como um modelo de espaços de módulos de superfícies de Riemann de genus zero que aparecem na teoria de cordas abertas-fechadas estudadas por Zwiebach [Zw98]. Também apresentamos os 2-operads de compactificações de Fulton-MacPherson e introduzimos o 2-operad de Barratt-Eccles, uma variação colorida do bem conhecido operad de Barratt-Eccles. Na terceira sessão desse capítulo apresentamos a estrutura modelo nas categorias de 2-operads e suas álgebras. Na quarta sessão apresentamos a construção das resoluções de Boardman-Vogt e mostramos que as resoluções dos operads de Fulton-MacPherson nos dão resoluções cofibrantes dos operads dos queijos suíços, estendendo um resultado de Hoefel para a versão não-reduzida desses 2-operads [HoE11]. Temos que a resolução de Boardman-Vogt do 2-operad de Barratt-Eccles é um $2 E_{\infty}$-operad.

O resultado principal é provado no quinto capítulo. Primeiro provamos uma versão relativa do teorema de aproximação. Apresentamos então alguns resultados técnicos sobre a compatibilidade da realização geométrica com os funtores $\Omega_{2}^{N}, \Sigma_{2}^{N}$ e monadas associadas a 2-operads. Esses resultados então são usados para provar os teoremas de reconhecimento relativos.

Livernet mostrou que ao contrário dos operads dos pequenos $N$-cubos $C^{N}$ os 2-operads $S C^{N}$ não são formais [Li15]. No apêndice mostramos que a demonstração da Livernet pode ser adaptada para mostrar a não-formalidade de $\mathcal{S} C_{\text {Vor }}^{2}$, uma variação do operad dos 2-queijos suíços em que a parte aberta não é reduzida. Questões de formalidade são de grande interesse no estudo de operads e suas aplicações. Kontsevich usou o 2-operad dos $N$-queijos suíços para descrever ações de $C^{\bullet}\left(C^{N}\right)$-álgebras em $C^{\bullet}\left(C^{N-1}\right)$-álgebras e a formalidade do operad dos pequenos cubos no seu trabalho de quantização por deformação [Ko99, Ta03]. Willwacher mostrou que versões estendidas desses 2-operads também não são formais [Wi17]. Relacionado ao trabalho de Kontsevich, Kajiura e Stasheff introduziram Algebras Homotópicas Abertas-Fechadas (OCHA) e Pares de Leibniz Fortemente Homotópicos (SHLP) em [KS06], que são álgebras sobre operads que podem ser obtidos da homologia do 2-operads dos queijos suíços, como foi demonstrado por Hoefel em [HoE07] e por Hoefel e Livernet em [HL12]. Os 2-operads dos queijos suíços foram estudados por diversos autores recentemente. Idrissi encontrou um modelo de $S C^{2}$ na categoria de grupóides [Id17], e no geral Quesney encontrou modelos para $S C^{N}$ na categoria de conjuntos e os usou para exibir modelos de espaços de laços relativos em dimensão 2 [Qu15]. Ducoulombier provou um teorema de reconhecimento relativo no contexto de espaços de imersões e de espaços cosimpliciais em [Du16] e [Du17]. 



\section{Capítulo 1}

\section{Categorias modelo}

Quillen introduziu categorias modelo em [Qu67] como uma abstração da estrutura na categoria de espaços topológicos que nos permite construir a sua categoria homotópica. Exemplos clássicos de categorias modelo são a categoria de conjuntos simpliciais, cuja categoria homotópica é equivalente à categoria homotópica de espaços topológicos, e a categoria de complexos de cadeia de $k$-módulos de um anel comutativo $k$, cuja categoria homotópica é a categoria derivada no sentido da teoria de álgebra homológica. Devido a esse último exemplo a teoria de categorias modelo é as vezes descrito como álgebra homotópica. Uma estrutura modelo em uma categoria bicompleta é composta por três classes de morfismos distinguidos, as equivalências fracas, cofibrações e fibrações, que satisfazem algumas condições. As equivalências fracas satisfazem condições para que exista uma localização da categoria nessa classe de morfismos. As fibrações e cofibrações satisfazem condições de fatoração e levantamento compatíveis com as equivalências fracas que nos permitem definir relações de homotopia entre morfismos e distinguir objetos da categoria nos quais a relação de homotopia se comporta bem, o que nos permite definir uma categoria homotópica que é uma localização localmente pequena da categoria modelo nas equivalências fracas. Para uma descrição detalhada de categorias modelo veja [GJ09, Hi09, HoM07], e para a definição equivalente usada aqui veja [Ri09].

Na primeira sessão damos as definições de uma estrutura modelo e provamos algumas propriedades básicas. Definimos os objetos bifibrantes de uma categoria modelo, que como veremos depois são os objetos da categoria homotópica da categoria modelo.

Na segunda sessão definimos as relações de homotopia à esquerda e à direita em uma categoria modelo, definimos equivalências homotópicas e apresentamos a demonstração do teorema de Whitehead, que nos diz que um morfismo entre objetos bifibrantes é uma equivalência fraca se e somente se for uma equivalência homotópica. Esse resultado nos permite definir a categoria homotópica de uma categoria modelo como a categoria cujos objetos são os objetos bifibrantes e cujos morfismos são as classes de equivalência homotópicas de morfismos entre eles.

$\mathrm{Na}$ terceira sessão apresentamos condições para que um funtor entre categorias modelo seja derivável, ou seja que ele induza um funtor nas categorias homotópicas, e condições em pares de 
funtores entre categorias modelo tais que eles induzam adjunções entre as categorias homotópicas. A condição encontrada na literatura e a mais usada em aplicações é a de que os funtores formem uma adjunção de Quillen, i.e. que eles sejam funtores adjuntos tais que o adjunto à direita preserva fibrações e o adjunto à esquerda preserva cofibrações. Hovey comenta em [HoM07] que para obter uma adjunção nas categorias homotópicas basta que o adjunto à direita preserve objetos fibrantes e equivalências fracas entre eles e que o adjunto à esquerda preserve objetos cofibrantes e equivalências fracas entre eles. Exemplos de adjunções de Quillen fracas que não são adjunções de Quillen são as adjunções $\left(\Sigma_{2}^{N} \dashv \Omega_{2}^{N}\right)$ do capítulo 3. Definimos aqui uma versão mais fraca ainda, denotada por quasi-adjunção fraca de Quillen, onde ao invés de assumir que os funtores são adjuntos eles admitem um span e um cospan natural que satisfazem condições análogas a unidade e counidade de uma adjunção, de forma que eles induzem uma unidade e uma counidade dos funtores derivados. Os funtores $\Omega^{N}$ e $B^{N}$ do principio de reconhecimento clássico e os funtores $\Omega_{2}^{N}$ e $B_{2}^{N}$ do princípio do reconhecimento relativo do capítulo 5 são exemplos.

Na quarta sessão damos a definição de uma estrutura modelo cofibrantemente gerada, que é uma estrutura modelo gerada por um conjunto de cofibrações e cofibrações triviais, e damos condições para que essas estruturas modelo possam ser transferidas via adjunções para outras categorias bicompletas. Em particular o teorema de transferência de estruturas modelo nos dá a estrutura modelo estrita de espectros do capítulo 3 e a estrutura modelo de 2-operads e álgebras sobre um 2-operad do capítulo 4.

Na quinta sessão descrevemos a estrutura modelo de uma categoria sobre um objeto e de uma categoria sob um objeto de uma categoria modelo. Em particular isso nos dá uma estrutura modelo na categoria pontuada de um categoria modelo, que é a categoria sob o objeto terminal.

Na sexta sessão descrevemos a estrutura modelo de categorias de funtores em categorias de Reedy generalizadas. Em particular isso nos dá uma estrutura modelo na categoria de objetos simpliciais de uma categorias modelo, que precisaremos considerar quando usarmos a construção bar, e na categoria de $\mathbb{S}$-objetos e $2 \mathbb{S}$-objetos, que são as estruturas subjacentes de operads e 2operads do capítulo 4.

Em algumas estruturas modelo equivalências fracas são fechadas por pushouts ao longo de cofibrações ou por pullbacks ao longo de fibrações, e chamamos tais categorias de próprias a esquerda e a direita respectivamente, e dizemos que ela é própria se ela satisfaz ambas as condições. Essa condição a mais fornece mais controle sobre as equivalências fracas. Na sétima sessão descrevemos algumas condições para que uma estrutura modelo seja própria.

Na oitava sessão apresentamos localizações de Bousfield. Uma localização de Bousfield de uma categoria modelo é uma estrutura modelo na mesma categoria cujas equivalências fracas contenham as equivalências fracas da categoria modelo original. Se a nova estrutura modelo tem as mesmas cofibrações dizemos que ela é uma localização à esquerda e se ela tem as mesmas fibrações dizemos que ela é uma localização à direita. Uma localização de Bousfield à esquerda define uma subcategoria reflexiva da categoria homotópica, i.e. uma subcategoria plena cuja in- 
clusão admite um adjunto à esquerda, e uma localização de Bousfield à direita define uma subcategoria coreflexiva da categoria homotópica, i.e. uma subcategoria plena cuja inclusão admite um adjunto à direita. Apresentamos um método de localização análogo ao método de Bousfield e Friedlander via monadas idempotentes de Quillen [BF78] modificado para podermos aplicá-lo na quasi-monada associada a uma quasi-adjunção de Quillen fraca. Usaremos localizações de Bousfield à esquerda para definir a estrutura modelo estável de espectros no capítulo 3 e a estrutura modelo de álgebras grouplike do capítulo 5. Também usaremos uma localização de Bousfield à direita para definir uma estrutura modelo de espectros conectivos no capítulo 5.

Na nona sessão usamos os resultados sobre localizações de Bousfield para definir quasi-adjunções de Quillen fracas idempotentes, e mostramos que essa estrutura induz equivalências entre subcategorias (co)reflexivas das categorias homotópicas adjuntas. O princípio de reconhecimento infinito clássico e o princípio de reconhecimento relativo infinito provado no capítulo 5 são casos particulares desse teorema.

\subsection{Estrutura modelo}

Dada uma categoria $\mathcal{T}$ e uma subclasse de morfismos $W$ as vezes queremos tratar esses morfismos como se eles fossem isomorfismos, ou seja queremos construir uma localização de $\mathcal{T}$ em relação a $W$. Por exemplo em Top muitas vezes queremos tratar equivalências homotópicas fracas como se elas fossem isomorfismos mesmo elas não tendo necessariamente inversas, nem mesmo a menos de homotopia. Como veremos na próxima sessão os axiomas de categorias modelo nos dão condições gerais em que é possível construir categorias homotópicas, que são localizações da categoria modelo nas equivalências fracas. Primeiramente é necessário que $W$ satisfaça as seguintes condições.

Definição 1.1.1. Seja $\mathcal{T}$ uma categoria. Uma subclasse de morfismos $W$ de $\mathcal{T}$ é uma classe de equivalências fracas se:

i) $W$ contém todos os isomorfismos de $\mathcal{T}$,

ii) $W$ satisfaz a propriedade dois-de-três: para todos $X, Y, Z \in \mathcal{T}, f \in \mathcal{T}(X, Y)$ e $g \in \mathcal{T}(Y, Z)$ se dois dos morfismos em $\{f, g, g f\}$ pertencem a $W$, então o terceiro também pertence.

Poderíamos formalmente adicionar inversas aos elementos de $W$ em uma categoria com equivalências fracas $\mathcal{T}$, porém a localização de $\mathcal{T}$ construída assim pode não ser localmente pequena. A teoria de categorias modelo contorna esse problema assumindo a existência de duas classes de morfismos de $\mathcal{T}$ e dois sistemas de fatoração funtoriais de morfismos que satisfazem propriedades de levantamento compatíveis com $W$. Essa estrutura extra define relações de equivalência nos conjuntos de morfismos entre certos objetos que podemos usar para definir uma categoria homotópica bem comportada. 
Definição 1.1.2. Seja $\mathcal{T}$ uma categoria, $\iota \in \mathcal{T}(A, X)$ e $p \in \mathcal{T}(E, B)$. O morfismo $\iota$ tem a propriedade de levantamento à esquerda em relação a $p$, e equivalentemente $p$ tem a propriedade de levantamento à direita em relação a $\iota$, se para todo quadrado comutativo $(k, l) \in \mathcal{T}^{\rightarrow}(\iota, p)$ existe um levantamento $H$ de $(k, l)$, i.e., um morfismo $H \in \mathcal{T}(X, E)$ tal que $H \iota=k$ e $p H=l$.

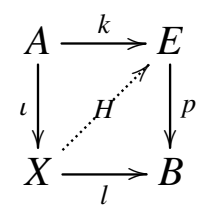

Denotamos que $\iota$ e $p$ satisfazem essa propriedade por $\iota \nabla p$. Dada uma subclasse de morfismos $M$ em $\mathcal{T}$ a classe de todos os morfismos com a propriedade de levantamento à esquerda em relação aos morfismos em $M$ é denotada por ${ }^{\square} M$, e a classe de todos os morfismos com a propriedade de levantamento à direita em relação aos morfismos em $M$ é denotada por $M^{\square}$.

Definição 1.1.3. Seja $\mathcal{T}$ uma categoria e $f \in \mathcal{T}(X, Y)$. Uma fatoração de $f$ é um par de morfismos componíveis $\left(f^{\prime}, f^{\prime \prime}\right) \in \bigsqcup_{K \in \mathcal{T}} \mathcal{T}(X, K) \times \mathcal{T}(K, Y)$ tal que $f=f^{\prime \prime} f^{\prime}$.

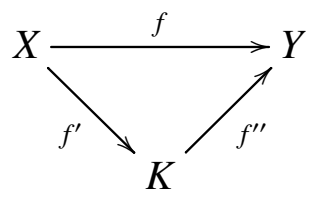

Denotamos por $\operatorname{Fat}(f)$ a classe de fatorações de $f$.

Denotamos por Dom, Codom : $\mathcal{T}^{\rightarrow} \rightarrow \mathcal{T}$ os funtores de domínio e codomínio respectivamente.

Definição 1.1.4. Seja $\mathcal{T}$ uma categoria. Um sistema de fatoração fraco em $\mathcal{T}$ é um par de subclasses de morfismos $L$ e $R$ de $\mathcal{T}$ equipados com um funtor Fat $_{L, R}: \mathcal{T} \rightarrow \rightarrow \mathcal{T}$ e um par de transformações naturais $-_{L}$ : Dom $\Rightarrow \mathrm{Fat}_{L, R} \mathrm{e}-_{R}:$ Fat $_{L, R} \Rightarrow$ Codom tais que

i) $L={ }^{\square} R$ e $R=L^{\square}$;

ii) Para todo $f \in \mathcal{T}^{\rightarrow}$ temos que $\left(f_{L}, f_{R}\right) \in \operatorname{Fat}(f), f_{L} \in L$ e $f_{R} \in R$.

Um sistema de fatoração nos dá uma fatoração funtorial de morfismos em um morfismo em $L$ seguido de um morfismo em $R$.

Definição 1.1.5. Seja $\mathcal{T}$ uma categoria. Uma estrutura modelo em $\mathcal{T}$ é uma tripla de subclasses de morfismos $(W, C, F)$ de $\mathcal{T}, W$ as equivalências fracas, $C$ as cofibrações e $F$ as fibrações, equipadas com um par de funtores Fat ${ }_{C, F \cap W}: \mathcal{T}^{\rightarrow} \rightarrow \mathcal{T}$ e Fat ${ }_{C \cap W, F}: \mathcal{T}^{\rightarrow} \rightarrow \mathcal{T}$ e uma quadrupla de transformações naturais $-_{C}:$ Dom $\Rightarrow$ Fat $_{C, F \cap W},-_{F \cap W}:$ Fat $_{C, F \cap W} \Rightarrow$ Codom, ${ }_{C \cap W}$ : Dom $\Rightarrow$ Fat $_{C \cap W, F} \mathrm{e}-{ }_{F}:$ Fat $_{C \cap W, F} \Rightarrow$ Codom tais que:

i) $W$ é uma classe de equivalências fracas de $\mathcal{T}$; 
ii) $\left(C, F \cap W ;\right.$ Fat $\left._{C, F \cap W},{ }_{C},-{ }_{F \cap W}\right)$ e $\left(C \cap W, F\right.$; Fat $\left.{ }_{C \cap W, F},-{ }_{C \cap W},{ }_{F}\right)$ são sistemas de fatorações fracos de $\mathcal{T}$.

Morfismos em $C \cap W$ são chamados cofibrações triviais e morfismos em $W \cap F$ de fibrações triviais.

Uma estrutura modelo em uma categoria $\mathcal{T}$, além de nos dar uma classe de equivalências fracas, nos permite fatorar funtorialmente todo morfismo $f$ em uma cofibração $f_{C}$ seguida de uma fibração trivial $f_{F \cap W}$ ou em uma cofibração trivial $f_{C \cap W}$ seguida de uma fibração $f_{F}$, sendo que as cofibrações tem a propriedade de levantamento em relação às fibrações triviais e as cofibrações triviais tem a propriedade de levantamento em relação às fibrações. Uma categoria modelo é uma categoria que admite todos os limites e colimites pequenos equipada com uma estrutura modelo.

Definição 1.1.6. Uma categoria modelo é uma categoria bicompleta $\mathcal{T}$ equipada com uma estrutura modelo.

No geral nos referimos à uma estrutura modelo em uma categoria modelo $\mathcal{T}$ simplesmente como $(W, C, F)$ ou $\left(W_{\mathcal{T}}, C_{\mathcal{T}}, F_{\mathcal{T}}\right)$ quando queremos explicitar a categoria subjacente, e deixamos implícitos os funtores e transformações naturais dos sistemas de fatorações fracos. Usamos a convenção que em diagramas as cofibrações são denotadas por flechas que começam com uma curva $\hookrightarrow$, fibrações por flechas com ponta dupla $\rightarrow$ e equivalências fracas por flechas marcadas com um til $\stackrel{\sim}{\rightarrow}$.

Estudamos agora algumas propriedades de fechamento das classes distinguidas de uma categoria modelo por construções categoriais usuais.

Definição 1.1.7. Sejam $\mathcal{T}$ uma categoria, $f \in \mathcal{T}(X, Y)$ e $f^{\prime} \in \mathcal{T}\left(X^{\prime}, Y^{\prime}\right)$. Dizemos que $f^{\prime}$ é uma retração de $f$ se existe $\left(\left(i_{0}, i_{1}\right),\left(r_{0}, r_{1}\right)\right) \in \mathcal{T}^{\rightarrow}\left(f^{\prime}, f\right) \times \mathcal{T}^{\rightarrow}\left(f, f^{\prime}\right) \cap \operatorname{Fat}\left(\left(1_{X^{\prime}}, 1_{Y^{\prime}}\right)\right)$.

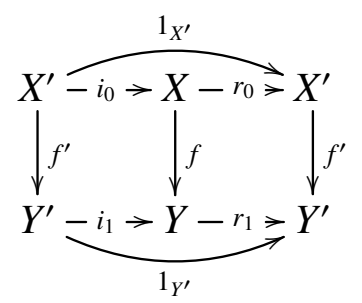

Denotamos por $\operatorname{Ret}(f)$ a classe das retrações de $f$.

A seguinte relação entre a propriedade de levantamento, fatorações e retrações é útil.

Lema 1.1.8 (Argumento da retração). Sejam $\mathcal{T}$ uma categoria, $f \in \mathcal{T}(X, Y) e(\iota, p) \in$ Fat $(f)$. Se $f \square p$ então $f \in \operatorname{Ret}(\iota)$. Se $\iota \nabla f$ então $f \in \operatorname{Ret}(p)$.

Demonstração: Provamos somente a primeira afirmação, já que a segunda segue por um argumento dual. Por hipótese temos um levantamento $H$ de $\left(\iota, 1_{Y}\right)$ tal que $\left(\left(1_{X}, H\right),\left(1_{X}, p\right)\right) \in$ 
$\operatorname{Fat}\left(\left(1_{X}, 1_{Y}\right)\right)$ nos dá que $f \in \operatorname{Ret}(\iota)$.
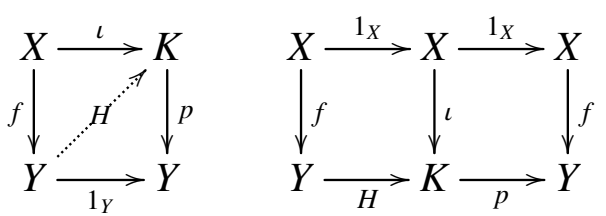

As classes de morfismos definidas por propriedades de levantamento à esquerda, como por exemplo as cofibrações e as cofibrações triviais em uma categoria modelo, em particular são fechadas por composições transfinitas, que podemos definir como colimites de funtores em ordinais.

Definição 1.1.9. Um ordinal $\kappa$ é um conjunto linearmente ordenado (e portanto possui uma estrutura de categoria) tal que todo subconjunto de elementos possui um elemento minimal.

Uma $\kappa$-sequência em uma categoria $\mathcal{T}$ é um funtor $X_{-} \in \mathcal{T}^{\kappa}$ que preserva colimites.

A composição transfinita de uma $\kappa$-sequência é o morfismo $X_{0<\kappa} \in \mathcal{T}\left(X_{0}, \operatorname{colim}_{\alpha \in \kappa} X_{\alpha}\right)$.

Lema 1.1.10. Seja $\mathcal{T}$ uma categoria bicompleta e $M$ uma classe de morfismos em $\mathcal{T}$.

i) ${ }^{\square}$ M e $M^{\square}$ contém todos os isomorfismos;

ii) ${ }^{\square}$ M e $M^{\square}$ são fechadas por composições $e^{\square} M$ é fechada por composições transfinitas;

iii) ${ }^{\square}$ M e $M^{\square}$ são fechadas por retrações;

iv) ${ }^{\square} M$ é fechada por pushouts e $M^{\square}$ é fechada por pullbacks;

v) ${ }^{\square} M$ é fechada por coprodutos e $M^{\square}$ é fechada por produtos.

\section{Demonstração:}

i) Sejam $f \in \mathcal{T}(X, Y)$ um isomorfismo, $m \in M(K, L)$ e $(k, l) \in \mathcal{T}^{\rightarrow}(f, m)$. Então $k f^{-1} \in \mathcal{T}(Y, K)$ é um levantamento de $(k, l)$.

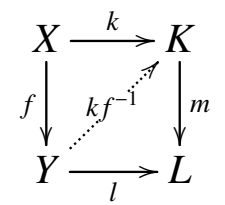

Logo todo isomorfismo está contido em ${ }^{\square} M$. Que todo isomorfismo está contido em $M^{\square}$ segue por um argumento dual.

ii) Sejam $\iota_{0} \in{ }^{\square} M\left(A_{0}, A_{1}\right)$ e $\iota_{1} \in{ }^{\square} M\left(A_{1}, X\right), m \in M(K, L)$ e $(k, l) \in \mathcal{T}^{\rightarrow}\left(\iota_{1} \iota_{0}, m\right)$. Um levantamento $H_{0} \in \mathcal{T}\left(A_{1}, K\right)$ de $\left(k, l_{1}\right)$ induz um levantamento $H_{1} \in \mathcal{T}(X, K)$ de $\left(H_{0}, l\right)$ que também é um 
levantamento de $(k, l)$.

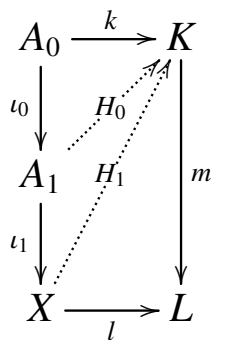

Logo ${ }^{\square} M$ é fechado por composições. Que $M^{\square}$ é fechado por composições segue por um argumento dual.

Que ${ }^{\square} M$ é fechado por composições transfinitas segue do fato que composições transfinitas são colimites de $\kappa$-sequências e os levantamentos dessas sequências construídos como acima formam um cocone que induzem um levantamento do colimite.

iii) Sejam $\iota \in{ }^{\square} M(A, X), \iota^{\prime} \in \mathcal{T}\left(A^{\prime}, X^{\prime}\right),\left(i_{0}, i_{1}\right) \in \mathcal{T}^{\rightarrow}\left(\iota^{\prime}, \iota\right)$ e $\left(r_{0}, r_{1}\right) \in \mathcal{T}^{\rightarrow}\left(\iota, \iota^{\prime}\right), m \in M(K, L)$ e $(k, l) \in \mathcal{T}^{\rightarrow}\left(\iota^{\prime}, m\right)$ tais que $\left(\left(i_{0}, i_{1}\right),\left(r_{0}, r_{1}\right)\right) \in \operatorname{Fat}\left(\left(1_{A^{\prime}}, 1_{X^{\prime}}\right)\right)$. Um levantamento $H \in \mathcal{T}(X, K)$ de $\left(k r_{0}, l r_{1}\right)$ induz um levantamento $H i_{1} \in \mathcal{T}\left(X^{\prime}, K\right)$ de $(k, l)$.

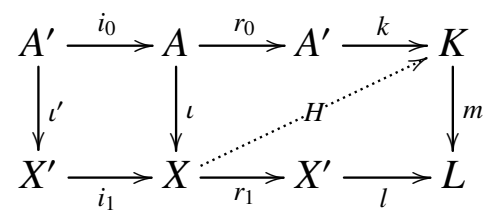

Logo ${ }^{\square} M$ é fechado por retrações. Que $M^{\square}$ é fechado por retrações segue por um argumento dual.

iv) Sejam $\iota \in{ }^{\square} M(A, X), m \in M(K, L), f \in \mathcal{T}(A, B), k \in \mathcal{T}(B, K)$ e $l \in \mathcal{T}(X, L)$ tais que $m k f=l \iota$. Um levantamento $H \in \mathcal{T}(X, K)$ de $(k f, l)$ induz um levantamento $(H, k) \in \mathcal{T}\left(X \sqcup_{A} B, K\right)$ de $(k,(l, m k))$.

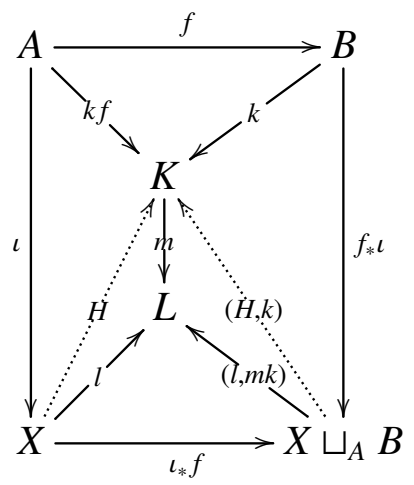

$\operatorname{Logo}^{\square} M$ é fechado por pushouts. Que $M^{\square}$ é fechado por pullbacks segue por um argumento dual.

v) Sejam $S \in$ Set, $\iota^{S} \in \prod_{s \in S}{ }^{\square} M\left(A^{s}, X^{s}\right), m \in M(K, L)$ e $\left(k^{S}, l^{S}\right) \in \prod_{s \in S} \mathcal{T}^{\rightarrow}\left(\iota^{s}, m\right)$. Então levantamentos $H^{S} \in \prod_{s \in S} \mathcal{T}\left(X^{s}, K\right) \operatorname{dos}\left(k^{S}, l^{S}\right)$ induzem um levantamento $H^{S} \in \mathcal{T} \rightarrow\left(\sqcup_{s \in S} X^{s}, K\right)$ 
de $\left(k^{S}, l^{S}\right)$.

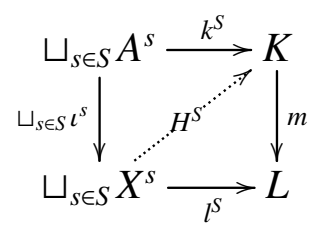

$\operatorname{Logo}^{\square} M$ é fechado por coprodutos. Que $M^{\square}$ é fechado por produtos segue por um argumento dual.

Proposição 1.1.11. Seja $\mathcal{T}$ uma categoria modelo.

i) W, C e F contém todos os isomorfismos;

ii) $W, C$ e $F$ são fechadas por composições; $C$ e $C \cap W$ são fechadas por composições transfinitas;

iii) $W, C$ e $F$ são fechadas por retrações;

iv) $C$ e $C \cap W$ são fechadas por pushouts; $F$ e $F \cap W$ são fechadas por pullbacks;

v) $C$ e $C \cap W$ são fechadas por coprodutos; $F$ e $F \cap W$ são fechadas por produtos.

Demonstração: A única propriedade que não é um caso particular de um dos casos do lema 1.1.10, a propriedade dois-de-três em $W$ ou a hipótese que $W$ contém todos os isomorfismos é que $W$ é fechada por retrações. Seja $f \in W(X, Y)$ e $f^{\prime} \in \operatorname{Ret}(f)$. Como Fat ${ }_{C, F \cap W}$ é um funtor e ${ }_{C}$ uma transformação natural temos $f_{C}^{\prime} \in \operatorname{Ret}\left(f_{C}\right)$. Pela propriedade dois-de-três $f_{C} \in C \cap W$ e portanto $f_{C}^{\prime} \in C \cap W$ pelo lema 1.1.10.iii.

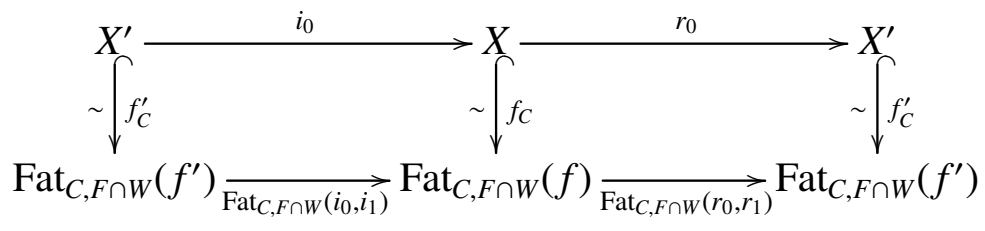

Logo pela propriedade dois-de-três $f^{\prime} \in W$ e assim $W$ é fechado por retrações.

Uma categoria bicompleta em particular possui um objeto inicial $\emptyset$ e um objeto final $*$ (bem definidos a menos de isomorfismo).

Definição 1.1.12. Sejam $\mathcal{T}$ uma categoria modelo e $X \in \mathcal{T}$. Dizemos que $X$ é cofibrante se $\emptyset_{X} \in$ $C(\emptyset, X)$, e que $X$ é fibrante se $*_{X} \in F(X, *)$. Dizemos que $X$ é bifibrante se ele for ambos cofibrante e fibrante. Denotamos as subclasses de objetos cofibrantes, fibrantes e bifibrantes de $\mathcal{T}$ por $\mathcal{T}_{\text {Cof }}$, $\mathcal{T}_{\text {Fib }}$ e $\mathcal{T}_{\text {Bif }}$ respectivamente.

Objetos bifibrantes são importantes pois, como veremos na próxima sessão, equivalências fracas entre objetos bifibrantes possuem inversas a menos de homotopia. 
Definição 1.1.13. Seja $X$ um objeto de uma categoria modelo $\mathcal{T}$. Uma resolução cofibrante de $X$ é um $\operatorname{par}(\bar{X}, c) \in \mathcal{T}_{\text {Cof }} \times W(\bar{X}, X)$. Uma resolução fibrante de $X$ é um $\operatorname{par}(\widetilde{X}, f) \in \mathcal{T}_{\text {Fib }} \times W(X, \widetilde{X})$.

As estruturas de fatorações fracas de categorias modelo nos dão resoluções (co,bi)fibrantes funtoriais.

Proposição 1.1.14. Seja $\mathcal{T}$ uma categoria modelo. O funtor

$$
\begin{aligned}
& \text { Cof }: \mathcal{T} \rightarrow \mathcal{T} \\
& X \mapsto \text { Fat }_{C, W \cap F}\left(\emptyset_{X}\right)
\end{aligned}
$$

e a fibração trivial natural cof $:=\left(\emptyset_{-}\right)_{F \cap W}:$ Cof $\Rightarrow I d_{\mathcal{T}}$ são tais que para todo $X \in \mathcal{T}$ o par $\left(\mathrm{Cof} X, \operatorname{cof}_{X}\right)$ é uma resolução cofibrante de $X$.

O funtor

$$
\begin{aligned}
\text { Fib }: \mathcal{T} & \rightarrow \mathcal{T} \\
X & \mapsto \operatorname{Fat}_{C \cap W, F}\left(*_{X}\right)
\end{aligned}
$$

e a cofibração trivial natural fib $:=\left(*_{-}\right)_{C \cap W}: I d_{\mathcal{T}} \Rightarrow$ Fib são tais que para todo $X \in \mathcal{T}$ o par $\left(\mathrm{Fib} X, \mathrm{fib}_{X}\right)$ é uma resolução fibrante de $X$.

Demonstração: Segue direto das definições.

Lema 1.1.15. Os funtores $\mathrm{Cof} e \mathrm{Fib}$ preservam equivalências fracas.

Demonstração: Isso segue do fato que cof e fib são equivalências fracas naturais e pela propriedade dois-de-três.

\subsection{Homotopia e categoria homotópica}

Nessa sessão mostraremos como construir a categoria homotópica de uma categoria modelo. Em uma categoria modelo $\mathcal{T}$ temos duas noções de homotopia entre morfismos paralelos $f, g \in$ $\mathcal{T}(X, Y)$, uma dada por objetos cilíndricos e outra dada por objetos de caminhos. Veremos que se $X$ for cofibrante e $Y$ fibrante essas noções coincidem e definem uma relação de equivalência.

Definição 1.2.1. Seja $\mathcal{T}$ uma categoria modelo e $X \in \mathcal{T}$. Um objeto cilíndrico de $X$ é um objeto $\operatorname{Cil}(X)$ equipado com um par de morfismos componíveis $\left(\left(\iota_{0}, \iota_{1}\right), p\right) \in C(X \sqcup X, \operatorname{Cil}(X)) \times$ $W(\operatorname{Cil}(X), X)$ tal que $\left(\left(\iota_{0}, \iota_{1}\right), p\right) \in \operatorname{Fat}\left(\Delta_{X}\right), \operatorname{com} \Delta_{X}:=\left(1_{X}, 1_{X}\right) \in \mathcal{T}(X \sqcup X, X)$.

$$
X \sqcup X \stackrel{\left(\iota_{0}, l_{1}\right)}{\longrightarrow} \operatorname{Cil}(X) \stackrel{p}{\sim} X
$$

Um objeto de caminhos de $X$ é um objeto $\operatorname{Cam}(X)$ equipado com um par de morfismos componíveis $\left(\iota,\left(p_{0}, p_{1}\right)\right) \in W(X, \operatorname{Cam}(X)) \times F(\operatorname{Cam}(X), X \times X)$ tal que $\left(\iota,\left(p_{0}, p_{1}\right)\right) \in \operatorname{Fat}\left(\nabla_{X}\right)$, com 
$\nabla_{X}:=\left(1_{X}, 1_{X}\right): X \rightarrow X \times X$.

$$
X \stackrel{\iota}{\sim} \operatorname{Cam}(X) \stackrel{\left(p_{0}, p_{1}\right)}{\longrightarrow} X \times X
$$

As vezes iremos nos referir a $\operatorname{Cil}(X)$ como o objeto cilíndrico de $X$ e $\operatorname{Cam}(X)$ como o objeto de caminhos de $X$ e deixaremos o resto da estrutura implícita. Os sistemas de fatoração da estrutura modelo garantem a existência desses objetos já que $\operatorname{Fat}_{C, W \cap F}\left(\Delta_{X}\right)$ é um objeto cilíndrico de $X$ e Fat $_{C \cap W, F}\left(\nabla_{X}\right)$ é um objeto de caminhos de $X$.

Lema 1.2.2. Sejam $\mathcal{T}$ uma categoria modelo, $X \in \mathcal{T}, \operatorname{Cil}(X)$ um objeto cilíndrico de $X$ e Cam $(X)$ um objeto de caminhos de $X$. Se $X \in \mathcal{T}_{\text {Cof }}$ então $\iota_{0}, \iota_{1} \in C \cap W(X, \operatorname{Cil}(X))$. Se $X \in \mathcal{T}_{\text {Fib }}$ então $p_{0}, p_{1} \in F \cap W(\operatorname{Cam}(X), X)$.

Demonstração: Provamos apenas a primeira afirmação, já que a segunda é formalmente dual. Como as inclusões da propriedade universal de coprodutos $i_{X}^{0}, i_{X}^{1} \in \mathcal{T}(X, X \sqcup X)$ são pushouts de duas cópias de $\emptyset_{X}$ temos pelo lema 1.1.10.iv que $i_{X}^{0}, i_{X}^{1} \in C$. Como pelo lema 1.1.10.ii a classe de cofibrações é fechada por composição temos que $\iota_{0}, \iota_{1} \in C(X, \operatorname{Cil}(X))$.

Como $p \iota_{0}=1_{X}=p \iota_{1}, p \in W(\operatorname{Cil}(X), X)$ e $1_{X} \in W(X, X)$ a propriedade dois-de-três implica que $\iota_{0}, \iota_{1} \in W$

Definição 1.2.3. Sejam $\mathcal{T}$ uma categoria modelo e $f, g \in \mathcal{T}(X, Y)$. Uma homotopia à esquerda de $f$ em $g$, denotada por $H: f \simeq_{L} g$, é um objeto cilíndrico $\operatorname{Cil}(X)$ de $X$ e um morfismo $H \in$ $\mathcal{T}(\operatorname{Cil}(X), Y)$ tal que $H\left(\iota_{0}, \iota_{1}\right)=(f, g)$.

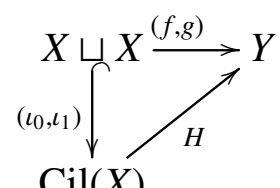

$\operatorname{Cil}(X)$

Uma homotopia à direita de $f$ em $g$, denotada por $H: f \simeq_{R} g$, é um objeto de caminhos $\operatorname{Cam}(Y)$ de $Y$ e um morfismo $H \in \mathcal{T}(X, \operatorname{Cam}(Y))$ tal que $\left(p_{0}, p_{1}\right) H=(f, g)$.

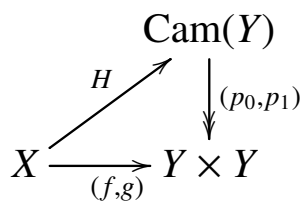

Dizemos que $f$ é homotópico à g pela esquerda se existe uma homotopia à esquerda $H$ : $f \simeq_{L} g$, e denotamos essa relação por $f \simeq_{L} g$, que $f$ é homotópico à $g$ pela direita se existe uma homotopia à direita $H: f \simeq_{R} g$, e denotamos essa relação por $f \simeq_{R} g$, e que $f$ é homotópico à $g$ se $f$ for homotópico à $g$ pela esquerda e pela direita, e denotamos essa relação por $f \simeq g$.

Definição 1.2.4. Sejam $\mathcal{T}$ uma categoria modelo e $f \in \mathcal{T}(X, Y)$. Dizemos que $f$ é uma equivalência homotópica se existir um morfismo $g \in \mathcal{T}(Y, X)$ tal que $g f \simeq 1_{X}$ e $f g \simeq 1_{Y}$. Nesse 
caso dizemos que $g$ é uma inversa homotópica de $f$. Denotamos a subclasse de equivalências homotópicas de $\mathcal{T}$ por $\mathcal{T}_{\simeq}$.

Lema 1.2.5. Sejam $\mathcal{T}$ uma categoria modelo e $f, g \in \mathcal{T}(X, Y)$. Se $X \in \mathcal{T}_{\text {Cof }}$ e $f \simeq_{L} g$ então $f \simeq_{R} g$. Se $Y \in \mathcal{T}_{\text {Fib }}$ e $f \simeq_{R} g$ então $f \simeq_{L} g$.

Demonstração: Provamos apenas a primeira afirmação, já que a segunda é formalmente dual. Sejam $H_{L}: f \simeq_{L} g$ com $H_{L} \in \mathcal{T}(\operatorname{Cil}(X), Y)$ e $\operatorname{Cam}(Y)$ um objeto cilíndrico de $Y$. Pelo lema 1.2.2 existe um levantamento $H: \mathcal{T}(\operatorname{Cil}(X), \operatorname{Cam}(X))$ de $\left(\iota f,\left(f p, H_{L}\right)\right) \in \mathcal{T}^{\rightarrow}\left(\iota_{0},\left(p_{0}, p_{1}\right)\right)$.

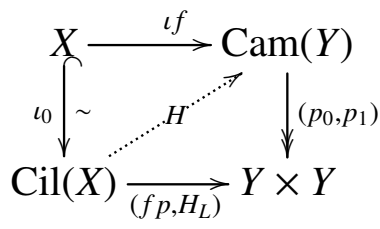

Temos então que $H_{R}:=H \iota_{1}: f \simeq_{R} g$.

Proposição 1.2.6. Sejam $\mathcal{T}$ uma categoria modelo e $X, Y \in \mathcal{T}$. Se $X \in \mathcal{T}_{\text {Cof }}$ então $\simeq_{L}$ é uma relação de equivalência em $\mathcal{T}(X, Y)$.

Se $Y \in \mathcal{T}_{\text {Fib }}$ então $\simeq_{R}$ é uma relação de equivalência em $\mathcal{T}(X, Y)$.

Se $X \in \mathcal{T}_{\text {Cof }}$ e $Y \in \mathcal{T}_{\text {Fib }}$ então $\simeq, \simeq_{L} e \simeq_{R}$ coincidem em $\mathcal{T}(X, Y)$ e são uma relação de equivalência.

Demonstração: Que as relações coincidem na terceira afirmação segue do lema 1.2.5. Provamos então apenas a primeira afirmação, já que a segunda é formalmente dual.

A relação $\simeq_{L}$ é reflexiva já que para todo $f \in \mathcal{T}(X, Y)$ sempre temos a homotopia à esquerda $f p: f \simeq_{L} f$.

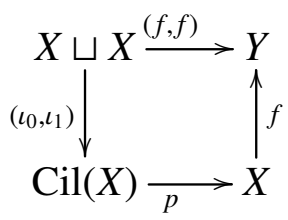

A relação $\simeq_{L}$ é simétrica pois $H \in \mathcal{T}(\operatorname{Cil}(X), Y)$ satisfaz $H\left(\iota_{0}, \iota_{1}\right)=(f, g)$ se e somente se $H\left(\iota_{1}, \iota_{0}\right)=(g, f)$.

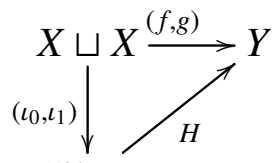

$\operatorname{Cil}(X)$

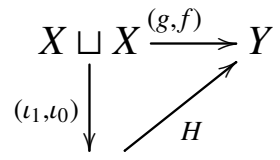

$\operatorname{Cil}(X)$

Sejam $\operatorname{Cil}(X)$ e $\operatorname{Cil}^{\prime}(X)$ objetos cilíndricos de $X$. Considere o seguinte diagrama, no qual o 
quadrado do meio é um pushout.

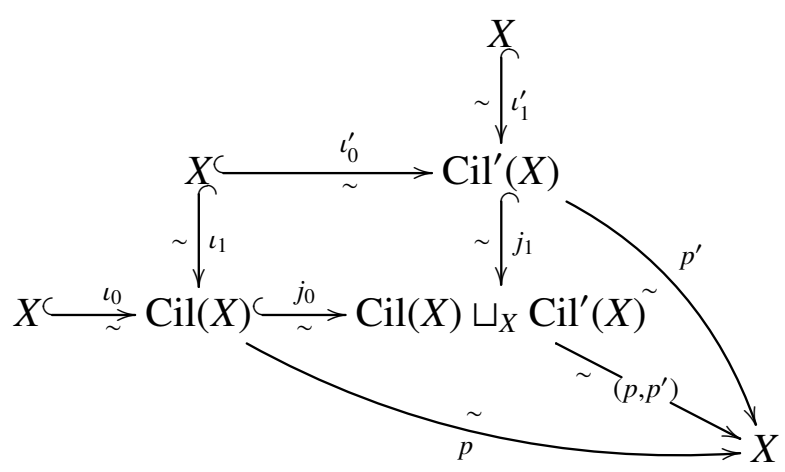

Como $\iota_{1}, \iota_{0}^{\prime} \in C \cap W$ pelo lema 1.2.2 e a proposição 1.1.10.iv) que $j_{0}, j_{1} \in C \cap W$, e portanto pela propriedade dois-de-três que $\left(p, p^{\prime}\right) \in W$. O morfismo $\left(j_{0} \iota_{0}, j_{1} \iota_{1}^{\prime}\right) \in \mathcal{T}\left(X \sqcup X, \operatorname{Cil}(X) \sqcup_{X} \operatorname{Cil}^{\prime}(X)\right)$ é igual a composição

$$
X \sqcup X \stackrel{\iota_{0} \sqcup 1_{X}}{\longrightarrow} \operatorname{Cil}(X) \sqcup X \stackrel{\left(j_{0}, j_{1} \iota_{1}^{\prime}\right)}{\longrightarrow} \operatorname{Cil}(X) \sqcup_{X} \operatorname{Cil}^{\prime}(X) .
$$

Denotando por $i_{X}^{1} \in \mathcal{T}(X, X \sqcup X)$ a inclusão do primeiro coproduto temos que $\iota_{0} \sqcup 1_{X}=i_{X *}^{1} \iota_{0}$, e portanto pela proposição 1.1.11.iv temos $\iota_{0} \sqcup 1_{X} \in C$. Logo como $\left(j_{0}, j_{1} \iota_{1}^{\prime}\right)=\left(\iota_{1} \sqcup 1_{X}\right)_{*}\left(\iota_{0}^{\prime}, \iota_{1}^{\prime}\right)$ então pela proposição 1.1.11.iv) também temos $\left(j_{0}, j_{1} \iota_{1}^{\prime}\right) \in C$. Portanto pela proposição 1.1.11.ii) temos $\left(j_{0} \iota_{0}, j_{1} \iota_{1}^{\prime}\right) \in C$. Assim

$$
X \sqcup X \stackrel{\left(j_{0} \iota_{0}, j_{1} \iota_{1}^{\prime}\right)}{\longrightarrow} \operatorname{Cil}(X) \sqcup_{X} \operatorname{Cil}^{\prime}(X) \stackrel{\left(p, p^{\prime}\right)}{\sim} X
$$

é um objeto cilíndrico de $X$.

Portanto a relação $\simeq_{L}$ é transitiva pois se $H: f \simeq_{L} g$ e $H^{\prime}: g \simeq_{L} h$ são dadas por $H \in$ $\mathcal{T}(\operatorname{Cil}(X), Y)$ e $H^{\prime} \in \mathcal{T}\left(\operatorname{Cil}^{\prime}(X), Y\right)$, então $\left(H_{L}, H_{L}^{\prime}\right) \in \mathcal{T}\left(\operatorname{Cil}(X) \sqcup_{X} \operatorname{Cil}^{\prime}(X), Y\right)$ é uma homotopia à esquerda de $f$ em $h$

Essa proposição nos permite fazer as seguintes definições:

Definição 1.2.7. Sejam $\mathcal{T}$ uma categoria modelo e $X, Y \in \mathcal{T}$. Se $X \in \mathcal{T}_{\text {Cof }}$ então $\mathcal{H} o_{L} \mathcal{T}(X, Y)$ é o conjunto de classes de homotopia à esquerda de morfismos de $\mathcal{T}(X, Y)$.

Se $Y \in \mathcal{T}_{\text {Fib }}$ então $\mathcal{H} o_{R} \mathcal{T}(X, Y)$ é o conjunto de classes de homotopia à direita de morfismos de $\mathcal{T}(X, Y)$.

Se $X \in \mathcal{T}_{\text {Cof }}$ e $Y \in \mathcal{T}_{\text {Fib }}$ então $\mathcal{H} o \mathcal{T}(X, Y)$ é o conjunto de classes de homotopia de morfismos de $\mathcal{T}(X, Y)$.

Em todos os casos denotamos a classe de homotopia de um morfismo $f \in \mathcal{T}(X, Y)$ por $[f]$.

Lema 1.2.8. Sejam $\mathcal{T}$ uma categoria modelo, $A \in \mathcal{T}_{\text {Cof }}$ e $p \in F \cap W(E, B)$. Então a função $p_{*} \in \operatorname{Set}\left(\mathcal{H} o_{L} \mathcal{T}(A, E), \mathcal{H} o_{L} \mathcal{T}(A, B)\right)$ com $p_{*}([f])=[p f]$ é um isomorfismo.

Sejam $Z \in \mathcal{T}_{\text {Fib }}$ e $\iota \in C \cap W(A, X)$. Então a função $\iota^{*} \in \operatorname{Set}\left(\mathcal{H} o_{R} \mathcal{T}(X, Z), \mathcal{H} o_{R} \mathcal{T}(A, Z)\right)$ com 
$\iota^{*}([f])=[f \iota]$ é um isomorfismo.

Demonstração: Provamos apenas a primeira afirmação, já que a segunda é formalmente dual. Que $p_{*}$ está bem definido segue do fato que se $H: f \simeq_{L} g$ for uma homotopia à esquerda então $p H: p f \simeq_{L} p g$ também é. Se $H: p f \simeq_{L} p g$ é uma homotopia à esquerda então um levantamento $\tilde{H} \in \mathcal{T}(\operatorname{Cil}(A), E)$ de $((f, g), H)$ é uma homotopia à esquerda de $f$ em $g$, e portanto $p_{*}$ é injetora.

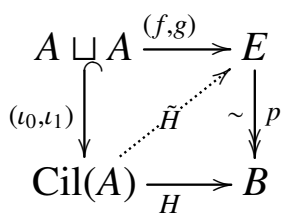

Por hipótese para todo $f \in \mathcal{T}(A, B)$ temos um levantamento $\tilde{f} \in \mathcal{T}(A, E)$ de $\left(\emptyset_{E}, f\right)$ tal que $p_{*}([\tilde{f}])=[f]$.

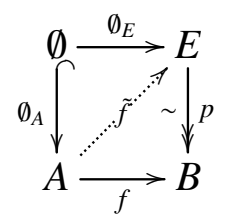

Portanto $p_{*}$ é sobrejetora

Lema 1.2.9. Sejam $\mathcal{T}$ uma categoria modelo, $h \in \mathcal{T}(W, X), f, g \in \mathcal{T}(X, Y)$ e $h^{\prime} \in \mathcal{T}(Y, Z)$. Se $Y \in \mathcal{T}_{\text {Fib }}$ e $f \simeq_{L} g$ então $f h \simeq_{L} g h$.

Se $X \in \mathcal{T}_{\text {Cof }}$ e $f \simeq_{R} g$ então $h^{\prime} f \simeq_{R} h^{\prime} g$.

Demonstração: Provamos apenas a primeira afirmação, já que a segunda é formalmente dual. Seja $H: f \simeq_{L} g$ uma homotopia à esquerda dada por $H \in \mathcal{T}(\operatorname{Cil}(X), Y)$. Temos então levantamentos $K \in \mathcal{T}\left(\operatorname{Cil}(W), \operatorname{Fat}_{C, W \cap F}(p)\right)$ de $\left(p_{C}\left(\iota_{0}, \iota_{1}\right)(h \sqcup h), h p^{\prime}\right) \in \mathcal{T}^{\rightarrow}\left(\left(\iota_{0}^{\prime}, \iota_{1}^{\prime}\right), p_{F \cap W}\right)$ e $\tilde{H} \in$ $\mathcal{T}\left(\operatorname{Fat}_{C, F \cap W}(p), Y\right) \operatorname{de}\left(H, *_{\mathrm{Fat}_{C, F \cap W}(p)}\right) \in \mathcal{T}^{\rightarrow}\left(p_{C}, *_{Y}\right)$.

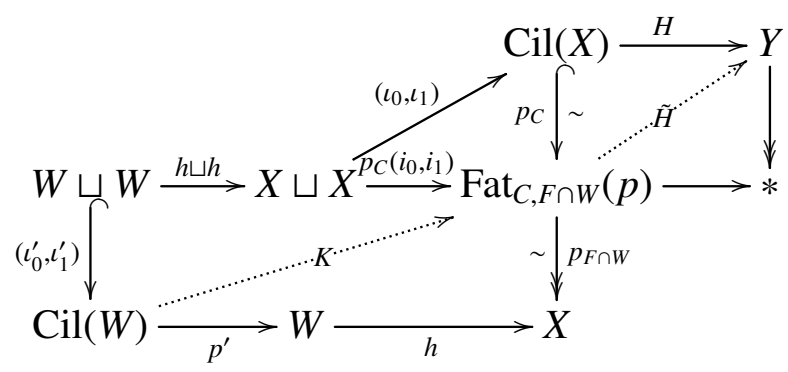

A composição dos levantamentos nos dá uma homotopia à esquerda $\tilde{H} K: f h \simeq_{L} g h$.

Proposição 1.2.10. Sejam $\mathcal{T}$ uma categoria modelo, $f \in \mathcal{T}_{\simeq}(X, Y)$ e $f^{\prime} \in \mathcal{T}_{\simeq}(Y, Z)$. Se $Y \in \mathcal{T}_{\text {Bif }}$ então $f^{\prime} f \in \mathcal{T}_{\simeq}$.

Demonstração: Isso é uma consequência direta do lema 1.2.9 e da definição de equivalência homotópica. 
Teorema 1.2.11. Seja $\mathcal{T}$ uma categoria modelo, $X \in \mathcal{T}_{\text {Cof }}, Y \in \mathcal{T}_{\text {Bif }}, Z \in \mathcal{T}_{\text {Fib }}, f, g \in \mathcal{T}(X, Y) e$ $h, k \in \mathcal{T}(Y, Z)$. Se $f \simeq g$ e $h \simeq k$ então $h f \simeq k g$, e portanto temos uma composição $\mathcal{H} o \mathcal{T}(X, Y) \times$ $\mathcal{H} o \mathcal{T}(Y, Z) \rightarrow \mathcal{H} o \mathcal{T}(X, Z)$ bem definida.

Demonstração: Isso é uma consequência direta do lema 1.2.9 e da proposição 1.2.6.

Teorema 1.2.12 (Whitehead). Sejam $\mathcal{T}$ uma categoria modelo, $X, Y \in \mathcal{T}_{\text {Bif }}$ e $f \in \mathcal{T}(X, Y)$. Então $f \in W$ se e somente se $f \in \mathcal{T}_{\simeq}$.

Demonstração: Considere a fatoração $\left(f_{C \cap W}, f_{F}\right) \in \operatorname{Fat}(f)$. Suponha que $f \in W$. Seja $r \in$ $\mathcal{T}\left(\operatorname{Fat}_{C \cap W, F}(f), X\right)$ um levantamento de $\left(1_{X}, *_{\operatorname{Fat}_{C \cap W, F}(f)}\right) \in \mathcal{T}^{\rightarrow}\left(f_{C \cap W}, *_{X}\right)$.

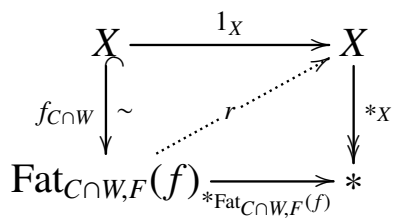

Por definição $r f_{C \cap W}=1_{X}$. Além disso pela proposição 1.1.11.ii) temos que $\operatorname{Fat}_{C \cap W, F}(f) \in \mathcal{T}_{\text {Bif }}$, e portanto pelo lema 1.2.8 e proposição 1.2.6 as igualdades $f_{C \cap W}^{*}\left(\left[f_{C \cap W} r\right]\right)=\left[f_{C \cap W} r f_{C \cap W}\right]=$ $\left[f_{C \cap W}\right]=f_{C \cap W}^{*}\left(\left[1_{\mathrm{Fat}_{C \cap W, F}(f)}\right]\right)$ implicam que $f_{C \cap W} r \simeq 1_{\mathrm{Fat}_{C \cap W, F}(f)}$. Assim $f_{C \cap W}$ é uma equivalência homotópica. Como pela propriedade dois-de-três $f_{F}$ também é uma equivalência fraca um argumento dual nos dá que $f_{F}$ também é uma equivalência homotópica. Logo $f \in \mathcal{T}_{\text {}}$ pela proposição 1.2.10.

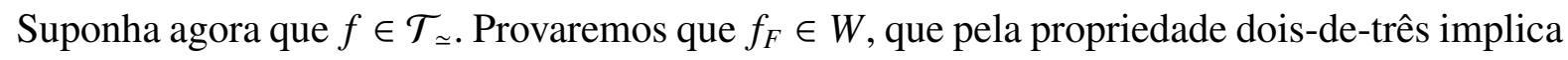
que $f \in W$. Por hipótese existe um morfismo $g \in \mathcal{T}(Y, X)$ e uma homotopia à esquerda $H: f g \simeq_{L}$ $1_{Y}$. Temos um levantamento $\tilde{H} \in \mathcal{T}\left(\operatorname{Cil}(Y), \operatorname{Fat}_{C \cap W, F}(f)\right) \operatorname{de}\left(f_{C \cap W} g, H\right) \in \mathcal{T}^{\rightarrow}\left(\iota_{0}, f_{F}\right)$.

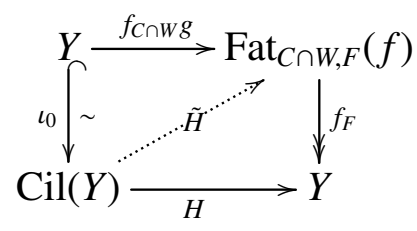

Portanto $\tilde{H}: f_{C \cap W} g \simeq_{L} \tilde{H} \iota_{1}$ e a comutatividade do diagrama nos dá que $f_{F} \tilde{H} \iota_{1}=1_{Y}$. Note que como $X \in \mathcal{T}_{\text {Cof }}$ e $Y \in \mathcal{T}_{\text {Fib }}$ pela proposição 1.1.11.ii) temos que Fat $_{C \cap W, F}(f) \in \mathcal{T}_{\text {Bif. }}$ Da primeira parte dessa demonstração temos uma inversa homotópica $r$ de $f_{C \cap W}$. Como $f=f_{F} f_{C \cap W}$ temos pelo lema 1.2.9 que $f r \simeq f_{F}$. Aplicando o lema 1.2.9 repetidamente obtemos uma sequência de homotopias

$$
\tilde{H} \iota_{1} f_{F} \simeq f_{C \cap W} g f_{F} \simeq f_{C \cap W} g f r \simeq f_{C \cap W} r \simeq 1_{\mathrm{Fat}_{C \cap W, F}(f)} .
$$

Portanto a existência da homotopia acima, o lema 1.2.2, a propriedade dois-de-três e o fato que 
$1_{\text {Fat }_{C \cap W, F}(f)} \in W$ implicam que $\tilde{H} \iota_{1} f_{F} \in W$. Como $f_{F} \in \operatorname{Ret}\left(\tilde{H} \iota_{1} f_{F}\right)$ pelo diagrama

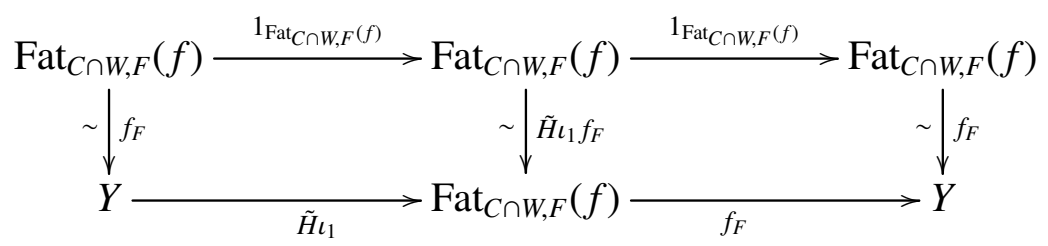

temos pela proposição 1.1.11.iii) que $f_{F} \in W$, e portanto pela propriedade dois-de-três $f \in W$.

Definição 1.2.13. A categoria homotópica $\mathcal{H} o \mathcal{T}$ de uma categoria modelo $\mathcal{T}$ é a categoria cuja classe de objetos é $\mathcal{T}_{\text {Bif }}$, e os morfismos entre $X, Y \in \mathcal{T}_{\text {Bif }}$ é $\mathcal{H} o \mathcal{T}(X, Y)$. O funtor de localização homotópica é definido por:

$$
\begin{aligned}
\pi: \mathcal{T} & \rightarrow \mathcal{H} o \mathcal{T} \\
X & \mapsto \operatorname{CofFib} X \\
f & \mapsto[\operatorname{CofFib} f]
\end{aligned}
$$

Teorema 1.2.14. Seja $\mathcal{T}$ uma categoria modelo. Então $\mathcal{H} o \mathcal{T}$ e $\pi: \mathcal{T} \rightarrow \mathcal{H}$ o $\mathcal{T}$ satisfazem a condição universal que se $F: \mathcal{T} \rightarrow \mathcal{U}$ é um funtor tal que $F w$ é um isomorfismo para todo $w \in W$, então existe um funtor $F_{\pi}: \mathcal{H} o \mathcal{T} \rightarrow \mathcal{U}$ equipado com um isomorfismo natural $\phi: F \Rightarrow F_{\pi} \pi$ tal que se $G: \mathcal{H} o \mathcal{T} \rightarrow \mathcal{U}$ é um funtor equipado com um isomorfismo natural $\psi: F \Rightarrow$ Gr então existe um único isomorfismo natural $\theta: F_{\pi} \Rightarrow G$ tal que $\psi=\theta_{\pi} \phi$.

Demonstração: Definimos

$$
\begin{aligned}
F_{\pi}: \mathcal{H} o \mathcal{T} & \rightarrow \mathcal{U} \\
X & \mapsto F X \\
{[f] } & \mapsto F f
\end{aligned}
$$

Esse funtor está bem definido pois se $f \simeq g$ então existe um objeto cilíndrico $\operatorname{Cil}(X)$ de $X$ e um morfismo $H \in \mathcal{T}(\operatorname{Cil}(X), Y)$ tal que $H i_{0}=f$ e $H i_{1}=g$. Como $p \in W(\operatorname{Cil}(X), X)$ por hipótese $F p$ é um isomorfismo, e como $p i_{0}=p i_{1}$ temos que $F i_{0}=F i_{1}$. $\log$ o $F f=F H i_{0}=F H i_{1}=F g$. Por construção $\phi:=F \operatorname{cof}_{\mathrm{Fib}}{ }^{-1} F$ fib : $F \Rightarrow F_{\pi} \pi$ é um isomorfismo natural. Se temos um outro funtor $G: \mathcal{H} o \mathcal{T} \rightarrow \mathcal{U}$ e um isomorfismo natural $\psi: F \Rightarrow G \pi$ então temos o isomorfismo natural $\theta:=G \mathrm{fib}^{-1} G \operatorname{cof}_{\mathrm{Fib}} \psi: F_{\pi} \Rightarrow G$, que pela comutatividade do diagrama

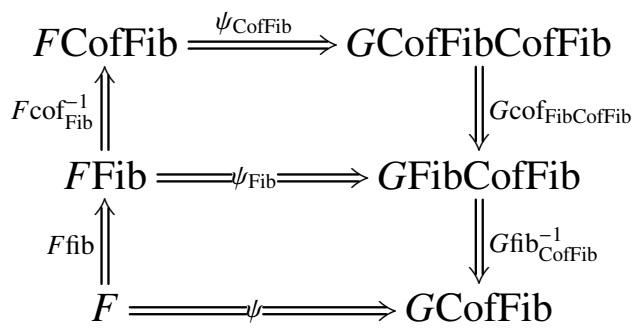


é o único tal que $\psi=\theta_{\pi} \phi$.

\subsection{Quasi-adjunções fracas de Quillen}

Funtores entre categorias modelo compatíveis com a estrutura modelo induzem funtores entre as categorias homotópicas.

Definição 1.3.1. Seja $\mathcal{T}$ uma categoria modelo, $\mathcal{U}$ uma categoria e $F: \mathcal{T} \rightarrow \mathcal{U}$ um funtor. Um funtor derivado à esquerda de $F$ é um funtor $\mathbb{L} F: \mathcal{H} o \mathcal{T} \rightarrow \mathcal{U}$ equipado com uma transformação natural $\phi: \mathbb{L} F \pi \Rightarrow F$ tal que se $G: \mathcal{H} o \mathcal{T} \rightarrow \mathcal{U}$ é um funtor equipado com uma transformação natural $\psi: G \pi \Rightarrow F$ então existe uma única transformação natural $\theta: G \Rightarrow \mathbb{L} F$ tal que $\psi=\phi \theta_{\pi}$.

Um funtor derivado à direita de $F$ é um funtor $\mathbb{R} F: \mathcal{H} o \mathcal{T} \rightarrow \mathcal{U}$ equipado com uma transformação natural $\phi: F \Rightarrow \mathbb{R} F \pi$ tal que se $G: \mathcal{H} o \mathcal{T} \rightarrow \mathcal{U}$ é um funtor equipado com uma transformação natural $\psi: F \Rightarrow G \pi$ então existe uma única transformação natural $\theta: \mathbb{R} F \rightarrow G$ tal que $\psi=\theta_{\pi} \phi$.

Quando um funtor derivado à esquerda ou à direita existe ele é único a menos de um isomorfismo natural único.

Proposição 1.3.2. Sejam $\mathcal{T}$ e $\mathcal{A}$ categorias modelo. Seja $S: \mathcal{T} \rightarrow \mathcal{A}$ um funtor que preserva objetos cofibrantes e equivalências fracas entre objetos cofibrantes. Então

$$
\begin{aligned}
\mathbb{L} S: \mathcal{H} o \mathcal{T} & \rightarrow \mathcal{H} o \mathcal{A} \\
X & \mapsto \operatorname{Fib} S X \\
{[f] } & \mapsto[\operatorname{Fib} S f]
\end{aligned}
$$

é um funtor derivado à esquerda de $\pi_{\mathcal{A}} S$ Cof.

Seja $\Lambda: \mathcal{A} \rightarrow T$ um funtor que preserva objetos fibrantes e equivalências fracas entre objetos fibrantes. Então

$$
\begin{aligned}
\mathbb{R} \Lambda: \mathcal{H} o \mathcal{A} & \rightarrow \mathcal{H} o \mathcal{T} \\
Y & \mapsto \operatorname{Cof} \Lambda Y \\
{[g] } & \mapsto[\operatorname{Cof} \Lambda g]
\end{aligned}
$$

é um funtor derivado à direita de $\pi_{\mathcal{T}} \Lambda$ Fib.

Demonstração: Provamos a primeira parte já que a segunda segue por um argumento dual.

Podemos definir $\phi:=\left[\operatorname{cof}_{\mathrm{FibS} C o f}\right]^{-1}[\mathrm{FibSCof} \text { fib }]^{-1}:$ FibS $\pi_{\mathcal{T}} \Rightarrow \pi_{\mathcal{A}} S$ Cof.

Sejam $G: \mathcal{H} o \mathcal{T} \rightarrow \mathcal{H} o \mathcal{A}$ um funtor e $\psi: G \pi_{\mathcal{T}} \Rightarrow \pi_{\mathcal{A}} S$ Cof uma transformação natural. Podemos definir $\theta:=[$ FibS cof $]\left[\operatorname{cof}_{\text {FibS } \operatorname{Cof}}\right] \psi G\left[\operatorname{cof}_{\text {Fib }}\right]^{-1} G[$ fib $]: G \Rightarrow$ Fib $S$. Pela comutatividade 
do diagrama

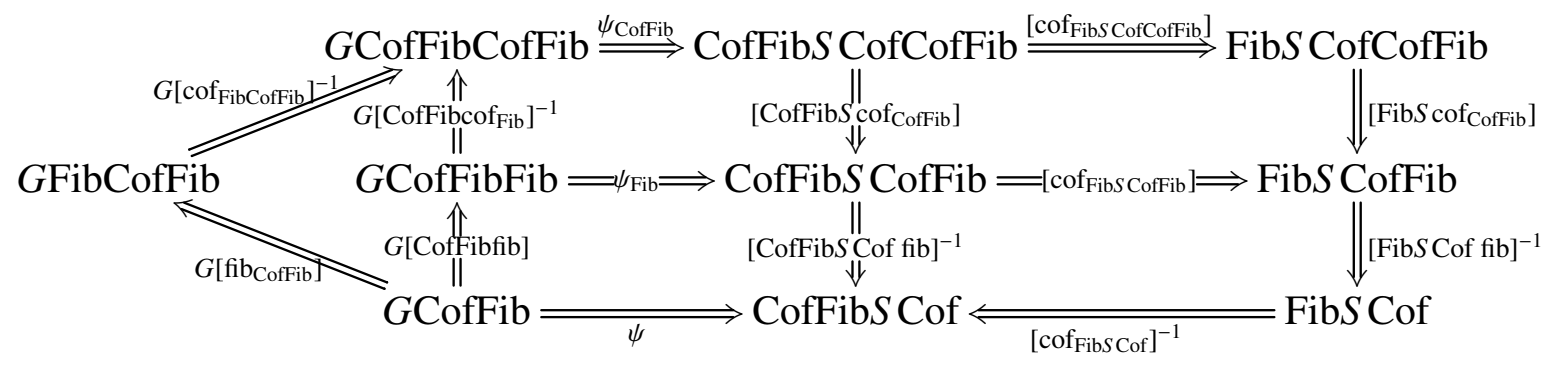

essa é a única transformação natural que satisfaz $\psi=\phi \theta_{\pi}$

Se os funtores na definição acima forem adjuntos então os funtores derivados definem uma adjunção entre as categorias homotópicas.

Definição 1.3.3. Sejam $\mathcal{T}$ e $\mathcal{A}$ categorias. Uma adjunção entre $\mathcal{T}$ e $\mathcal{A}$, denotada por $(S \dashv \Lambda)$ : $\mathcal{T} \rightleftharpoons \mathcal{A}$, é um par de funtores $S: \mathcal{T} \rightarrow \mathcal{A}$, o adjunto à esquerda, e $\Lambda: \mathcal{A} \rightarrow \mathcal{T}$, o adjunto à direita, equipados com transformações naturais $\eta: I d_{\mathcal{T}} \Rightarrow \Lambda S$, a unidade, e $\epsilon: S \Lambda \Rightarrow I d_{\mathcal{H}}$, a counidade, que satisfazem as equações de unidade-counidade $\epsilon_{S} S \eta=1_{S}$ e $\Lambda \epsilon \eta_{\Lambda}=1_{\Lambda}$.

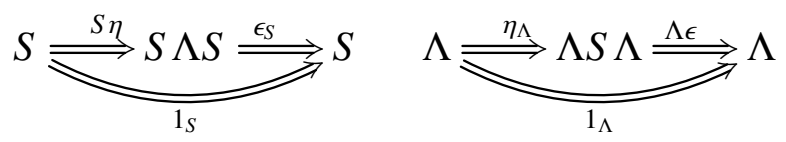

Se a unidade $\eta$ e a counidade $\epsilon$ forem isomorfismos naturais então $(S \dashv \Lambda)$ é uma equivalência de categorias.

Definição 1.3.4. Sejam $\mathcal{T}$ e $\mathcal{A}$ categorias modelo. Uma adjunção $(S \dashv \Lambda): \mathcal{T} \rightleftharpoons \mathcal{A}$ é uma adjunção de Quillen fraca se $S$ e $\Lambda$ satisfazem as seguintes condições:

i) Para todo $X \in \mathcal{T}_{\text {Cof }}$ temos que $S X \in \mathcal{A}_{\text {Cof }}$;

ii) Para todos $X, X^{\prime} \in \mathcal{T}_{\text {Cof }}$ e $f \in W_{\mathcal{T}}\left(X, X^{\prime}\right)$ temos que $S f \in W_{\mathcal{A}}\left(S X, S X^{\prime}\right)$;

iii) Para todo $Y \in \mathcal{A}_{\text {Fib }}$ temos que $\Lambda Y \in \mathcal{T}_{\text {Fib }}$;

iv) Para todos $Y, Y^{\prime} \in \mathcal{A}_{\text {Fib }}$ e $g \in W_{\mathcal{A}}\left(Y, Y^{\prime}\right)$ temos que $\Lambda f \in W_{\mathcal{T}}(\Lambda X, \Lambda Y)$.

É comum que adjunções entre categorias modelo satisfaçam condições mais fortes que as de adjunções de Quillen fracas.

Definição 1.3.5. Uma adjunção $(S \dashv \Lambda)$ entre categorias modelo é uma adjunção de Quillen se as seguintes condições equivalentes são satisfeitas:

- Para todo $\iota \in C_{\mathcal{T}}\left(\iota \in C_{\mathcal{T}} \cap W_{\mathcal{T}}\right)$ temos que $S \iota \in C_{\mathcal{A}}\left(S \iota \in C_{\mathcal{A}} \cap W_{\mathcal{A}}\right)$;

- Para todo $p \in F_{\mathcal{A}}\left(p \in F_{\mathcal{A}} \cap W_{\mathcal{A}}\right)$ temos que $\Lambda p \in F_{\mathcal{T}}\left(\Lambda p \in F_{\mathcal{T}} \cap W_{\mathcal{T}}\right)$; 
- Para todo $\iota \in C_{\mathcal{T}}$ temos que $S \iota \in C_{\mathcal{A}}$ e para todo $p \in F_{\mathcal{A}}$ temos que $\Lambda p \in F_{\mathcal{T}}$;

- Para todo $\iota \in C_{\mathcal{T}} \cap W_{\mathcal{T}}$ temos que $S \iota \in C_{\mathcal{A}} \cap W_{\mathcal{A}}$ e para todo $p \in F_{\mathcal{A}} \cap W_{\mathcal{A}}$ temos que $\Lambda p \in F_{\mathcal{T}} \cap W_{\mathcal{T}}$

O lema de Ken Brown implica que adjunções de Quillen são adjunções de Quillen fracas.

Lema 1.3.6. [Ken Brown] Seja $\mathcal{T}$ uma categoria modelo.

i) Existe um funtor Fat $_{\mathrm{Cof}, W}: \mathcal{T} \rightarrow \rightarrow \mathcal{T}$ e um par de transformações naturais $\iota^{\mathrm{Cof}, W}:$ Dom $\Rightarrow$

Fat $_{\mathrm{Cof}, W}$ e $p^{\mathrm{Cof}, W}:$ Fat $_{\mathrm{Cof}, W} \Rightarrow$ Codom tais que se $X, Y \in \mathcal{T}_{\text {Cof }}$ e $f \in W(X, Y)$ então temos $\left(\iota_{f}^{\mathrm{Cof}, W}, p_{f}^{\mathrm{Cof}, W}\right) \in \operatorname{Fat}(f), \iota_{f}^{\mathrm{Cof}, W} \in C \cap W, p_{f}^{\mathrm{Cof}, W} \in F \cap W$ e $p_{f}^{\mathrm{Cof}, W}$ admite uma inversa à direita que é uma cofibração trivial.

ii) Existe um funtor Fat $_{\mathrm{Fib}, W}: \mathcal{T}^{\rightarrow} \rightarrow \mathcal{T}$ e um par de transformações naturais $\iota^{\mathrm{Fib}, W}:$ Dom $\Rightarrow$ Fat $_{\mathrm{Fib}, W}$ e $p^{\mathrm{Fib}, W}:$ Fat $_{\mathrm{Fib}, W} \Rightarrow$ Codom tais que se $X, Y \in \mathcal{T}_{\text {Fib }}$ e $f \in W(X, Y)$ então temos $\left(\iota_{f}^{\mathrm{Fib}, W}, p_{f}^{\mathrm{Fib}, W}\right) \in \mathrm{Fat}(f), \iota_{f}^{\mathrm{Fib}, W} \in C \cap W, p_{f}^{\mathrm{Fib}, W} \in F \cap W e \iota_{f}^{\mathrm{Fib}, W}$ admite uma inversa à esquerda que é uma fibração trivial.

Demonstração: Provamos apenas a primeira parte, já que a segunda é dual. Seja $f \in \mathcal{T}(X, Y)$ e considere os morfismos estruturais de coproduto $i_{X}: X \rightarrow X \sqcup Y$ e $i_{Y}: Y \rightarrow X \sqcup Y$. Defina então $\operatorname{Fat}_{C o f, W}(f):=\operatorname{Fat}_{C, W \cap F}\left(f, 1_{Y}\right)$, que claramente pode ser estendido à um funtor, e defina as transformações naturais $\iota^{C, W \cap F}$ e $p^{C, W \cap F}$ por $\iota_{f}^{C, W \cap F}:=\left(f, 1_{Y}\right)_{C} i_{X}$ e $p_{f}^{C, W \cap F}:=\left(f, 1_{Y}\right)_{W \cap F}$.

$$
X \stackrel{i_{X}}{\longrightarrow} X \sqcup Y \stackrel{\left(f, 1_{Y}\right)_{C}}{\longrightarrow} \operatorname{Fat}_{C, W \cap F}\left(f, 1_{Y}\right) \stackrel{\left(f, 1_{Y}\right)_{W \cap F}}{\sim} Y
$$

Note que $\left(f, 1_{Y}\right)_{C} i_{Y}$ é uma inversa à direita de $\left(f, 1_{Y}\right)_{W \cap F}$.

Se $X, Y \in \mathcal{T}_{\text {Cof }}$ então pela proposição 1.1.11.iv) $i_{X}$ e $i_{Y}$ são cofibrações, e portanto pela proposição 1.1.11.ii) $\left(f, 1_{Y}\right)_{C} i_{X}$ e $\left(f, 1_{Y}\right)_{C} i_{Y}$ são cofibrações. Além disso se $f \in W$ pela propriedade doisde-três $\left(f, 1_{Y}\right)_{C} i_{X}$ e $\left(f, 1_{Y}\right)_{C} i_{Y}$ são equivalências fracas.

O seguinte corolário nos dá que adjunções de Quillen são adjunções de Quillen fracas.

Corolário 1.3.7. Sejam $\mathcal{T}$ e $\mathcal{A}$ categorias modelo. Se $S: \mathcal{T} \rightarrow \mathcal{A}$ é um funtor que manda cofibrações triviais entre objetos cofibrantes em equivalências fracas então $S$ preserva equivalências fracas entre objetos cofibrantes.

Se $\Lambda: \mathcal{A} \rightarrow \mathcal{T}$ é um funtor que manda fibrações triviais entre objetos fibrantes em equivalências fracas então $\Lambda$ preserva equivalências fracas entre objetos fibrantes.

Demonstração: Isso é uma consequência direta do lema 1.3.6 e da propriedade dois-de-três.

Nem todas adjunções entre as categorias homotópicas são induzidas por adjunções de Quillen fracas. Em particular precisaremos da seguinte generalização. 
Definição 1.3.8. Sejam $\mathcal{T}$ e $\mathcal{A}$ categorias modelo. Uma quasi-adjunção de Quillen fraca entre $\mathcal{T}$ e $\mathcal{A}$, denotada por $\left(S \dashv_{B, \bar{B}} \Lambda\right): \mathcal{T} \rightleftharpoons \mathcal{A}$ é uma quadrupla de funtores $S: \mathcal{T} \rightarrow \mathcal{A}$, o quasiadjunto de Quillen à esquerda, $\Lambda: \mathcal{A} \rightarrow \mathcal{T}$, o quasi-adjunto de Quillen à direita, $B: \mathcal{T} \rightarrow \mathcal{T} \mathrm{e}$ $\bar{B}: \mathcal{A} \rightarrow \mathcal{A}$ equipados com uma quadrupla de transformações naturais $\eta^{\prime}: B \Rightarrow I d_{\mathcal{T}}, \eta: B \Rightarrow \Lambda S$, $\epsilon^{\prime}: I d_{\mathcal{A}} \Rightarrow \bar{B}$ e $\epsilon: S \Lambda \Rightarrow \bar{B}$ tais que

i) Para todo $X \in \mathcal{T}_{\text {Cof }}$ temos que $S X \in \mathcal{A}_{\text {Cof }}$;

ii) Para todos $X, X^{\prime} \in \mathcal{T}_{\text {Cof }}$ e $f \in W_{\mathcal{T}}\left(X, X^{\prime}\right)$ temos que $S f \in W_{\mathcal{A}}\left(S X, S X^{\prime}\right)$;

iii) Para todo $Y \in \mathcal{A}_{\text {Fib }}$ temos que $\Lambda Y \in \mathcal{T}_{\text {Fib }}$;

iv) Para todos $Y, Y^{\prime} \in \mathcal{A}_{\mathrm{Fib}}$ e $g \in W_{\mathcal{A}}\left(Y, Y^{\prime}\right)$ temos que $\Lambda f \in W_{\mathcal{T}}(\Lambda X, \Lambda Y)$;

v) Para todo $X \in \mathcal{T}_{\text {Cof }}\left(X \in \mathcal{T}_{\text {Fib }}\right)$ temos que $B X \in \mathcal{T}_{\text {Cof }}\left(B X \in \mathcal{T}_{\text {Fib }}\right)$;

vi) Para todo $Y \in \mathcal{A}_{\mathrm{Cof}}\left(Y \in \mathcal{A}_{\mathrm{Fib}}\right)$ temos que $\bar{B} Y \in \mathcal{A}_{\mathrm{Cof}}\left(\bar{B} Y \in \mathcal{A}_{\mathrm{Fib}}\right)$;

vii) Para todo $X \in \mathcal{T}$ temos que $\eta_{X}^{\prime} \in W_{\mathcal{T}}$;

viii) Para todo $Y \in \mathcal{A}$ temos que $\epsilon_{Y}^{\prime} \in W_{\mathcal{A}}$;

ix) Para todo $X \in \mathcal{T}_{\text {Cof }}$ temos que $\epsilon_{S X} S \eta_{X} \simeq_{L} \epsilon_{S X}^{\prime} S \eta_{X}^{\prime}$;

x) Para todo $Y \in \mathcal{A}_{\text {Fib }}$ temos que $\Lambda \epsilon_{Y} \eta_{\Lambda Y} \simeq_{R} \Lambda \epsilon_{Y}^{\prime} \eta_{\Lambda Y}^{\prime}$.
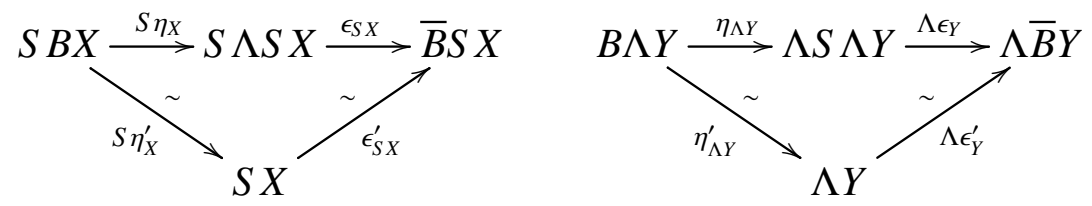

Note que se $B, \bar{B}, \eta^{\prime}$ e $\epsilon^{\prime}$ forem a identidade e assumirmos que as equações a menos de homtopia $i x)$ e $x$ ) são equações recuperamos a definição de adjunção de Quillen fraca. Note também que $B$ e $\bar{B}$ preservam equivalências fracas por $\eta^{\prime}$ e $\epsilon^{\prime}$ serem equivalências fracas naturai e pela propriedade dois-de-três. Precisaremos dessa generalização para lidar com a dependência da construção bar no teorema do reconhecimento.

Teorema 1.3.9. Uma quasi-adjunção de Quillen fraca $\left(S \dashv_{B, \bar{B}} \Lambda\right): \mathcal{T} \rightleftharpoons \mathcal{A}$ induz uma adjunção derivada nas categorias homotópicas $(\mathbb{L} S \dashv \mathbb{R} \Lambda): \mathcal{H} o \mathcal{T} \rightleftharpoons \mathcal{H}$ o $\mathcal{A}$.

Demonstração: Definimos a unidade da adjunção como a composição

$$
[\tilde{\eta}]:=I d_{\mathcal{H} o \mathcal{T}} \stackrel{\left[\eta^{\prime} \operatorname{cof}_{B}\right]^{-1}}{\Longrightarrow} \mathbb{R} B \stackrel{\left[\operatorname{Cof}\left(\Lambda \mathrm{fib}_{s} \eta\right)\right]}{=} \mathbb{R} \Lambda \llbracket S
$$

e a counidade como a composição

$$
[\tilde{\epsilon}]:=\mathbb{L} S \mathbb{R} \Lambda \stackrel{\left[\mathrm{Fib}\left(\epsilon S \operatorname{cof}_{\Lambda}\right)\right]}{=} \mathbb{L} \bar{B} \stackrel{\left[\mathrm{fib}_{\bar{B}} \epsilon^{\prime}\right]^{-1}}{\Longrightarrow} I d_{\mathcal{H} o \mathcal{A}}
$$


Que a primeira equação de counidade-unidade é satisfeita segue do fato que Fib preserva equivalências fracas, das hipóteses em $B, \bar{B}, S$ e $\Lambda$, do lema 1.2 .9 e da comutatividade do diagrama abaixo em $H o \mathcal{T}$.

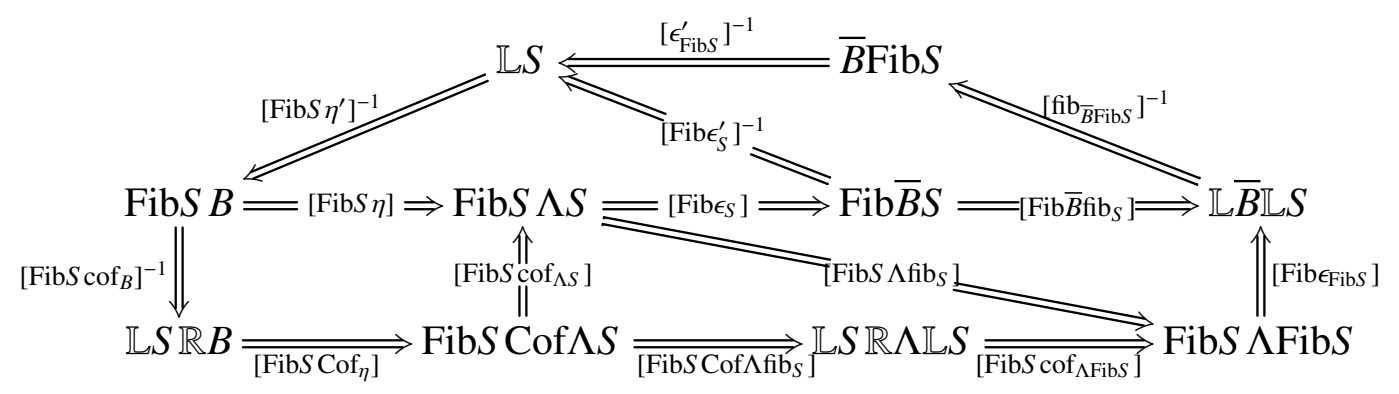

Note que os espaços Fib $S \Lambda S X$ e Fib $S \Lambda$ Fib $S X$ não são cofibrantes, e portanto eles não pertencem a $H o \mathcal{T}$, porém $[\tilde{\epsilon}]_{\mathbb{L} S}$ é definido via composições de morfismos que passam por FibS $\Lambda$ Fib $S X$ e a comutatividade do diagrama depende da comutatividade a menos de homotopia do primeiro diagrama de unidade-counidade que envolve Fib $S \Lambda S X$, e portanto eles estão incluídos para facilitar a visualização. Que vale a segunda equação segue por um argumento dual.

Teorema 1.3.10. Seja $\left(S \dashv_{B, \bar{B}} \Lambda\right): \mathcal{T} \rightleftharpoons \mathcal{A}$ uma quasi-adjunção de Quillen fraca. Se para todo $X \in \mathcal{T}_{\text {Cof }}$ e $Y \in \mathcal{A}_{\text {Fib }}$ temos que $\Lambda \operatorname{fib}_{S X} \eta_{X}$ e $\epsilon_{Y} S \operatorname{cof}_{\Lambda Y}$ são equivalências fracas então (LS $\dashv \mathbb{R} \Lambda$ ) : $\mathcal{H} o \mathcal{T} \rightleftharpoons \mathcal{H}$ of é uma equivalência de categorias.

Demonstração: Esse teorema é uma consequência direta das definições.

\subsection{Categorias modelo cofibrantemente geradas e transferência de estru- tura modelo}

O argumento do objeto pequeno é um teorema que nos dá condições nas quais um conjunto de morfismos $I$ de uma categoria $\mathcal{T}$ define um sistema de fatoração fraco. Em particular precisamos supor que os domínios dos morfismos em $I$ são pequenos no sentido dado pelas definições a seguir.

Definição 1.4.1. Seja $S \in$ Set. A cardinalidade $|S|$ de $S$ é o menor ordinal tal que existe uma bijeção $v \in \operatorname{Set}(|S|, S)$. Um cardinal é um ordinal $\kappa$ tal que $|\kappa|=\kappa$.

Seja $\gamma$ um cardinal. Um ordinal $\kappa$ é $\gamma$-filtrado se for um ordinal limite e, se $S \subset \kappa$ e $|S| \leq \gamma$, então $\sup S \in \kappa$.

Definição 1.4.2. Sejam $\mathcal{T}$ uma categoria cocompleta, $I$ uma classe de morfismos de $\mathcal{T}, A \in \mathcal{T}$ e $\gamma$ um cardinal. Dizemos que $A$ é $\gamma$-pequeno em relação à $I$ se, para todo ordinal $\gamma$-filtrado $\kappa$ e toda $\kappa$-sequência $X_{-} \in \mathcal{T}^{\kappa}$ tal que para cada $\alpha \in \kappa$ temos $X_{\alpha<\alpha+1} \in I\left(X_{\alpha}, X_{\alpha+1}\right)$, a função

$$
\operatorname{colim}_{\alpha \in \kappa} \mathcal{T}\left(A, X_{\alpha}\right) \rightarrow \mathcal{T}\left(A, \operatorname{colim}_{\alpha \in \kappa} X_{\alpha}\right)
$$

é uma bijeção. Dizemos que $A$ é pequeno em relação à $I$ se for $\gamma$-pequeno em relação à $I$ para algum $\gamma$, e que $A$ é pequeno se $A$ é pequeno em relação à $\mathcal{T}$. 
A definição a seguir é uma abstração da construção de CW-complexos relativos, i.e. de espaços obtidos colando discos pelo seu bordo em algum espaço dado.

Definição 1.4.3. Seja $\mathcal{T}$ uma categoria cocompleta e $I$ uma classe de morfismos de $\mathcal{T}$. Um morfismo $f \in \mathcal{T}(A, X)$ é um complexo I-celular relativo se $f$ for uma composição transfinita de pushouts de morfismos em $I$. Denotamos a classe de complexos $I$-celular relativo por $\operatorname{Cel}(I)$. Dizemos que $f$ é uma $I$-cofibração se for uma retração de um morfismo em $\operatorname{Cel}(I)$. Denotamos a classe de $I$-cofibrações por $\operatorname{Cof}(I)$.

Teorema 1.4.4 (Argumento do objeto pequeno). Seja $\mathcal{T}$ uma categoria cocompleta e I uma classe de morfismos de $\mathcal{T}$ tal que todos os domínios dos morfismos em I são pequenos em relação à $\operatorname{Cel}(I)$. Então temos um sistema de fatoração fraco $\left(\operatorname{Cof}(I), I^{\square} ; \operatorname{Fat}_{\operatorname{Cof}(I), I^{\square}},{ }^{\operatorname{Cof}(I)},{ }_{I^{\square}}\right)$.

Demonstração: Claramente $\operatorname{Cof}(I)^{\square} \subset I^{\square}$ já que $I \subset \operatorname{Cof}(I)$. Pelo lema 1.1.10 temos que $I^{\square} \subset \operatorname{Cof}(I)^{\square} . \log 0 \operatorname{Cof}(I)^{\square}=I^{\square}$

Por definição $I \subset{ }^{\square}\left(I^{\square}\right)$, e o lema 1.1.10 também nos da que $\operatorname{Cof}(I) \subset{ }^{\square}\left(I^{\square}\right)$.

Demonstramos agora que podemos funtorialmente fatorar os morfismos de $\mathcal{T}$ em complexo $I$ celulares relativos seguidos de um morfismo em $I^{\natural}$. Seja $f \in \mathcal{T}(X, Y)$. Definiremos recursivamente a fatoração de $f$. Defina $Z_{0}:=X, q_{0}:=f$ e $\bar{i}_{1}:=\sqcup_{(k, l) \in \bigsqcup_{i \in I} \mathcal{T} \rightarrow\left(i, q_{0}\right)} i$.

Note que definindo $K_{I / q_{0}}:=\sqcup_{(k, l) \in \bigsqcup_{i \in I} \mathcal{T} \rightarrow\left(i, q_{0}\right)} \operatorname{Dom} i, L_{I / q_{0}}:=\sqcup_{(k, l) \in \bigsqcup_{i \in I} \mathcal{T} \rightarrow\left(i, q_{0}\right)} \operatorname{Codom} i$ e $Z_{1}:=$ $L_{I / q_{0}} \sqcup_{K_{I / q_{0}}} Z_{0}$ os morfismos $(k, l)$ no indice dos coprodutos induzem um quadrado comutativo $\left(\bar{k}_{1}, \bar{l}_{1}\right) \in \mathcal{T} \rightarrow\left(\bar{i}_{1}, q_{0}\right)$, que por sua vez induz o diagrama comutativo

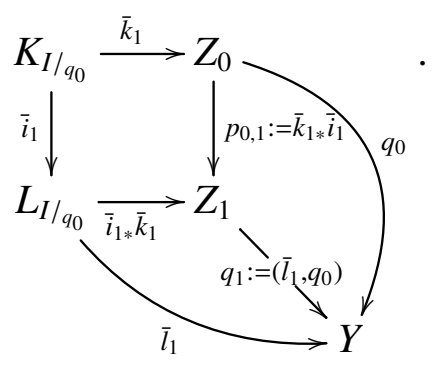

Isso nos dá uma fatoração $\left(p_{0,1}, q_{1}\right) \in \operatorname{Fat}(f)$. Podemos recursivamente construir fatorações $\left(p_{\alpha}, q_{\alpha}\right) \in$ Fat $(f)$ para cada ordinal $\alpha$, com $p_{\alpha}$ definido como a composição dos $p_{\beta, \beta+1}$ para $\beta \in \alpha$ e tomando colimites quando $\beta \in \alpha$ é um ordinal limite. Note que para todo ordinal $\alpha$ o morfismo $p_{\alpha} \in \operatorname{Cel}(I)$, já que podemos substituir um pushout de coprodutos de morfismos por uma composição possivelmente transfinita indexada pela cardinalidade do índice do coproduto. Seja $\kappa$ o menor ordinal tal que os domínios dos morfismos em $I$ são $\kappa$-pequenos em relação à $\operatorname{Cel}(I)$. Mostraremos agora que $p_{\kappa} \in I^{\square}$.

Dado $i \in I(K, L)$ e $(k, l) \in \mathcal{T}^{\rightarrow}\left(i, q_{\kappa}\right)$ a hipótese sobre $\kappa$ implica que existem $\alpha \in \kappa, k_{\alpha} \in$ $\mathcal{T}\left(K, Z_{\alpha}\right)$ e $p_{\alpha, \kappa} \in \mathcal{T}\left(Z_{\alpha}, Z_{\kappa}\right)$ tais que $\left(k_{\alpha}, p_{\alpha, \kappa}\right) \in \operatorname{Fat}(k)$. Como $Z_{\alpha+1}$ é definido como um pushout sobre quadrados comutativos incluindo $\left(k_{\alpha}, l\right)$ temos uma aplicação $p_{\alpha+1, \kappa} i_{L} \in \mathcal{T}\left(L, Z_{\kappa}\right)$ que é um 
levantamento de $(k, l)$.

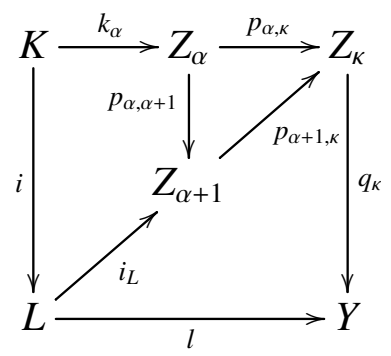

Essa construção é funtorial, e como $\operatorname{Cel}(I) \subset \operatorname{Cof}(I)$ podemos $\operatorname{definir}^{\operatorname{Fat}}{\operatorname{Cof}(I), I^{\natural}}(f):=Z_{\kappa}, f_{\operatorname{Cof}(I)}:=$ $p_{\kappa}$ e $f_{I^{\square}}:=q_{\kappa}$.

Falta portanto apenas provar que ${ }^{\square}\left(I^{\square}\right) \subset \operatorname{Cof}(I)$. Suponha que $f \in{ }^{\square}\left(I^{\square}\right)$. Por construção $\left(f_{\operatorname{Cof}(I)}, f_{I^{\Xi}}\right) \in \operatorname{Fat}(f), \operatorname{logo} f \in \operatorname{Ret}\left(f_{\operatorname{Cof}(I)}\right)$ pelo argumento da retração 1.1 .8 e portanto $f \in \operatorname{Cof}(I)$. $\operatorname{Assim} \operatorname{Cof}(I)={ }^{\square}\left(I^{\square}\right)$.

Esse teorema nos motiva a definir categorias modelo cujos sistemas de fatoração fracos são definidos por conjuntos de morfismos.

Definição 1.4.5. Uma estrutura modelo $(W, C, F)$ em uma categoria bicompleta $\mathcal{T}$ é cofibrantemente gerada se existem dois conjuntos de morfismos $I$, as cofibrações geradoras, e $J$, as cofibrações triviais geradoras, tais que

i) Os dominios dos morfismos em $I$ (resp. $J)$ são pequenos em relação à $\operatorname{Cel}(I)(\operatorname{resp} . \operatorname{Cel}(J))$;

ii) $\left(C, W \cap F ; \mathrm{Fat}_{C, W \cap F},-_{C},-{ }_{W \cap F}\right)=\left(\operatorname{Cof}(I), I^{\square} ; \mathrm{Fat}_{\operatorname{Cof}(I), I^{\square}},-{ }_{\operatorname{Cof}(I)},-I^{\square}\right)$;

iii) $\left(C \cap W, F ; \operatorname{Fat}_{C \cap W, F},-{ }_{C \cap W},-_{F}\right)=\left(\operatorname{Cof}(J), J^{\square} ; \operatorname{Fat}_{\operatorname{Cof}(J), J^{\square}},-{ }_{\operatorname{Cof}(J)},-J^{\square}\right)$.

O seguinte resultado nos dá condições para que um par de conjuntos de morfismos em uma categoria com equivalências fracas gere uma estrutura modelo via o argumento do objeto pequeno.

Proposição 1.4.6. Seja $\mathcal{T}$ uma categoria bicompleta e $W$ uma classe de equivalências fracas de $\mathcal{T}$. Se I e J são conjuntos de morfismos de $\mathcal{T}$ tais que:

i) Os dominios dos morfismos em I (resp. J) são pequenos em relação à Cel(I) (resp. Cel(J));

ii) ${ }^{\square}\left(J^{\square}\right) \subset{ }^{\square}\left(I^{\square}\right) \cap W$;

iii) $I^{\square} \subset J^{\square} \cap W$;

iv) Uma das seguintes condições é satisfeita:

a) ${ }^{\square}\left(I^{\square}\right) \cap W \subset{ }^{\square}\left(J^{\square}\right)$;

b) $J^{\square} \cap W \subset I^{\square}$.

Então $\mathcal{T}$ é uma categoria modelo cofibrantemente gerada aonde $W$ é a classe de equivalências fracas, I é o conjunto de cofibrações geradoras e J é o conjunto de cofibrações triviais geradoras. 
Demonstração: Pelo argumento do objeto pequeno basta provar que dadas as condições $i$ ), ii) e iii) então as condições $i v . a$ ) e $i v . b$ ) são equivalentes. Mostraremos que $i v . b$ ) implica $i v . a$ ); que $i v . a$ ) implica $i v . b$ ) segue por um argumento análogo. Suponha que $f \in{ }^{\square}\left(I^{\square}\right) \cap W$. Por $i$ ) e o argumento do objeto pequeno temos $\left(f_{\operatorname{Cof}(J)}, f_{J^{\Xi}}\right) \in \operatorname{Fat}(f)$. O argumento do objeto pequeno e a condição $i$ i) nos dá que $f_{\operatorname{Cof}(J)} \in W$, e assim a propriedade dois-de-três implica que $f_{J^{\square}} \in W$ e portanto por $i v . b$ ) temos que $f_{J^{\square}} \in I^{\square}$. Logo $f \square f_{J^{\square}}$ e pelo argumento da retração 1.1.8 temos que $f \in \operatorname{Ret}\left(f_{\operatorname{Cof}(J)}\right)$, e portanto que $f \in \operatorname{Cof}(J)$. Pelo argumento do objeto pequeno temos que $f \in{ }^{\square}\left(J^{\square}\right)$.

Uma das vantagens das estruturas modelo cofibrantemente geradas é que existem condições simples nas quais elas podem ser transferidas por adjunções.

Teorema 1.4.7. Sejam $\mathcal{T}$ uma categoria modelo cofibrantemente gerada com equivalências fracas $W$, fibrações $F$, cofibrações geradoras I e cofibrações triviais geradoras $J$, $\mathcal{A}$ uma categoria bicompleta e $(S \dashv \Lambda): \mathcal{T} \rightleftharpoons \mathcal{A}$ uma adjunção. Então condições suficientes para $\mathcal{A}$ admitir uma estrutura modelo cofibrantemente gerada com equivalências fracas $\Lambda^{-1}(W)$, fibrações $\Lambda^{-1}(F)$, cofibrações geradoras $S(I)$ e cofibrações triviais geradoras $S(J)$ são:

i) Os domínios dos morfismos em $S(I)$ (resp. $S(J))$ são pequenos em relação à Cel $(S(I))$ (resp. $\operatorname{Cel}(S(J)))$;

ii) $\operatorname{Cel}(S(J)) \subset \Lambda^{-1}(W)$.

Quando essas condições são satisfeitas $(S \dashv \Lambda)$ é uma adjunção de Quillen.

Demonstração: A adjunção $(S \dashv \Lambda)$ e o argumento do objeto pequeno implica que $\Lambda^{-1}(F)=$ $\Lambda^{-1}\left(J^{\square}\right)=S(J)^{\square} \mathrm{e}^{\square} \Lambda^{-1}(F) \cap{ }^{\square} \Lambda^{-1}(W)={ }^{\square} \Lambda^{-1}(W \cap F)={ }^{\square} \Lambda^{-1}\left(I^{\square}\right)={ }^{\square}\left(S(I)^{\square}\right)=\operatorname{Cof}(S(I))$. Mostramos então que $\Lambda^{-1}(W), S(I)$ e $S(J)$ satisfazem as condições da proposição 1.4.6.

Como todo funtor preserva isomorfismos e composições $\left(\mathcal{A}, \Lambda^{-1}(W)\right)$ é uma categoria com equivalências fracas.

A condição 1.4.6. $i$ é a hipótese $i)$. A adjunção $(S \dashv \Lambda)$ nos dá que $S(I)^{\square}=\Lambda^{-1}\left(I^{\square}\right)=\Lambda^{-1}(F \cap$ $W)=\Lambda^{-1}(F) \cap \Lambda^{-1}(W)=\Lambda^{-1}\left(J^{\square}\right) \cap \Lambda^{-1}(W)=S(J)^{\square} \cap \Lambda^{-1}(W)$, que nos dá que as condições 1.4.6.iii e 1.4.6.iv.b são satisfeitas.

A hipótese $i$ ), o lema 1.1.10.iii, o argumento do objeto pequeno e o fato que funtores preservam retrações implicam que ${ }^{\square}\left(S(J)^{\square}\right)=\operatorname{Cof}(S(J)) \subset \Lambda^{-1}(W)$. Como $I^{\square} \subset J^{\square}$ a adjunção $(S \dashv \Lambda)$ nos dá que $S(I)^{\square} \subset S(J)^{\square}$, o que por definição implica que ${ }^{\square}\left(S(J)^{\square}\right) \subset{ }^{\square}\left(S(I)^{\square}\right)$. Logo a condição 1.4.6.ii é satisfeita.

Por definição $\Lambda$ preserva fibrações e fibrações triviais, logo $(S \dashv \Lambda)$ é uma adjunção de Quillen.

Em muitos casos particulares o funtor $\Lambda$ preserva colimites filtrados, o que implica a primeira condição do teorema 1.4.7. O argumento dos objetos de caminho de Quillen nos dão condições que 
muitas vezes são simples de ser verificadas que implicam a segunda condição do teorema 1.4.7. $\mathrm{O}$ argumento depende do fato que as equivalências fracas satisfazem a propriedade dois-de-seis, demonstrado no lema a seguir.

Lema 1.4.8. Sejam $\mathcal{T}$ uma categoria modelo, $f \in \mathcal{T}(A, B), g \in \mathcal{T}(B, C)$ e $h \in \mathcal{T}(C, D)$. Se $g f, h g \in W$ então $f, g, h, h g f \in W$.

Demonstração: Suponha primeiro que $A, B, C, D \in \mathcal{T}_{\text {Bif. }}$ Pelo teorema de Whitehead 1.2.12 temos inversas homotópicas $k$ de $g f$ e $l$ de $h g$ tais que $k g l$ é uma inversa homotópica de $h g f$. Pelo teorema de Whitehead temos que $h g f \in W$ e portanto a propriedade dois-de-três implica que $h, g, f \in W$.

Suponha agora que $A, B, C, D \in \mathcal{T}$ são objetos quaisquer e considere o seguinte diagrama:

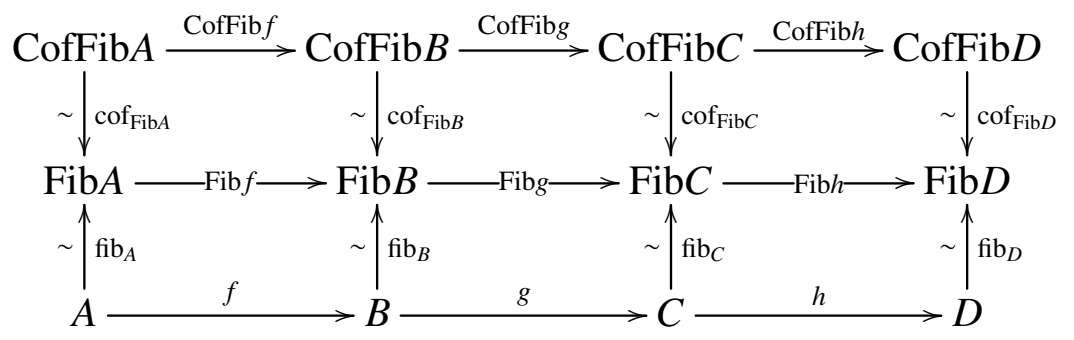

Então pela propriedade dois-de-três o caso em que todos os objetos são bifibrantes implica o caso geral.

Proposição 1.4.9. A condição 1.4.7.i) é satisfeita se $\Lambda$ preserva colimites filtrados e a condição 1.4.7.ii) é satisfeita se $\mathcal{A}$ admite um funtor de substituição fibrante e todo objeto fibrante admite um objeto de caminhos.

Demonstração: A condição $i$ ) segue de $\Lambda$ preservar colimites filtrados pois composições transfinitas são um caso particular de colimite filtrado.

Seja $\iota \in \operatorname{Cel}(S(J))(A, X)$. Temos que $\iota \in{ }^{\square} \Lambda^{-1}(F)$, portanto existe um levantamento $r \in$ $\mathcal{T}(X, \mathrm{Fib} A)$ de $\left(\mathrm{fib}_{A}, *_{X}\right) \in \mathcal{T}^{\rightarrow}\left(\iota, *_{\mathrm{Fib} A}\right)$.

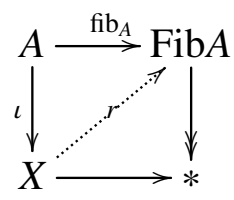

Temos portanto que $\Lambda r \Lambda \iota=\Lambda \mathrm{fib}_{A} \in W$. Provaremos que $\Lambda$ Fib $\iota \Lambda r \in W$, e portanto o lema 1.4.8 implica que $\Lambda \iota \in W$. Pela existência de um objeto de caminhos de Fib $X$ temos que existe um 
levantamento $H \in \mathcal{T}(X, \operatorname{Cam}(\operatorname{Fib} X))$ de $\left(i f i b_{X} \iota,\left(F_{i b} \iota r, f_{1}\right) \mathcal{T}\left(\iota,\left(p_{0}, p_{1}\right)\right)\right.$.

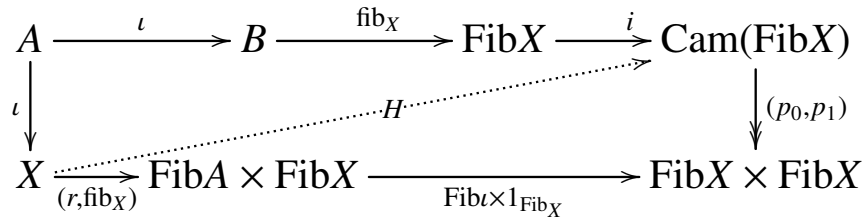

Portanto $\Lambda H$ nos dá que $\Lambda \mathrm{fib}_{X} \simeq_{R} \Lambda$ Fibı $\Lambda r$, e portanto pela propriedade dois-de-três e o lema 1.2.2 temos que $\Lambda$ Fibı $\Lambda r$ é uma equivalência fraca.

\subsection{Categorias pontuadas}

Uma estrutura modelo em uma categoria induz uma estrutura modelo nas categorias sobre e sob objetos. Estamos particularmente interessados em categorias pontuadas, i.e. em categorias sob o objeto terminal.

Definição 1.5.1. Seja $\mathcal{T}$ uma categoria e $Z \in \mathcal{T}$. A categoria sobre $Z$ é a categoria cujos objetos são morfismos $f \in \bigsqcup_{X \in \mathcal{T}} \mathcal{T}(X, Z)$ com codomínio $Z$, e cujos morfismos entre $f \in \mathcal{T}(X, Z)$ e $g \in \mathcal{T}(Y, Z)$ são morfismos $h \in \mathcal{T}(X, Y)$ tais que $g h=f$, i.e. o triângulo

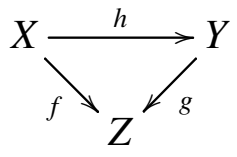

é comutativo. Denotamos essa categoria por $\mathcal{T}_{/ Z}$.

A categoria sob $Z$ é a categoria cujos objetos são morfismos $f \in \bigsqcup_{X \in \mathcal{T}} \mathcal{T}(Z, X)$ com domínio $Z$, e cujos morfismos entre $f \in \mathcal{T}(Z, X)$ e $g \in \mathcal{T}(Z, Y)$ são morfismos $h \in \mathcal{T}(X, Y)$ tais que $g=h f$, i.e. tais que o triangulo

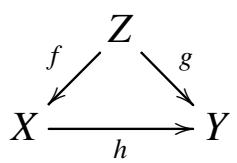

é comutativo. Denotamos essa categoria por $\mathcal{T}^{Z /}$.

Para $*$ um objeto final de $\mathcal{T}$ a categoria sob $*$ é a categoria pontuada de $\mathcal{T}$, que denotamos por $\mathcal{T}_{*}$. Denotamos o ponto base de um objeto pontuado por $X_{*} \in \mathcal{T}(*, X)$.

No teorema a seguir e no resto dessa tese usaremos que toda categoria com objetos iniciais, pushouts e colimites filtrados é cocompleta, e dualmente que toda categoria com objetos terminais, pullbacks e limites cofiltrados é completa [McL13, Teor. IX.1].

Teorema 1.5.2. Seja $\mathcal{T}$ uma categoria modelo e $Z \in \mathcal{T}$. Então $\mathcal{T}_{\mid Z}$ e $\mathcal{T}^{Z /}$ são categorias modelo nas quais um morfismo é uma equivalência fraca, cofibração ou fibração se o for em $\mathcal{T}$.

Demonstração: Provamos o teorema para $\mathcal{T}^{Z /}$. A afirmação para $\mathcal{T}_{/ Z}$ segue por um argumento 
dual. Temos que o funtor esquecimento $U: \mathcal{T}^{Z /} \rightarrow \mathcal{T}$ que manda $f \in \mathcal{T}(Z, X)$ no objeto $X$ cria limites, $\log _{0} \mathcal{T}^{Z /}$ é completa. Como $U$ também cria colimites filtrados e pushouts, e $1_{Z}$ é um objeto inicial de $\mathcal{T}^{Z /}$ temos que $\mathcal{T}^{Z /}$ é cocompleta.

Que a estrutura modelo de $\mathcal{T}$ induz estruturas modelos em $\mathcal{T}^{Z /}$ é trivial da descrição dos morfismos nessa categoria.

Proposição 1.5.3. Sejam $\mathcal{T}$ uma categoria modelo cofibrantemente gerada pelas cofibrações geradoras $I$ e as cofibrações triviais geradoras $J$ e $Z \in \mathcal{T}$. Então $\mathcal{T}^{Z /}$ é uma categoria modelo cofibrantemente gerada pelas cofibrações geradoras $I \sqcup Z$ e as cofibrações triviais geradoras $J \sqcup Z$.

Demonstração: $O$ funtor esquecimento $U: \mathcal{T}^{Z /} \rightarrow \mathcal{T}$ admite um adjunto a esquerda que manda objetos $X$ no morfismo $i_{Z} \in \mathcal{T}(Z, X \sqcup Z)$. Pela descrição dos colimites em $\mathcal{T}^{Z /}$ temos que $\operatorname{Cel}(I \sqcup Z) \subset \operatorname{Cel}(I)$ e $\operatorname{Cel}(J \sqcup Z) \subset \operatorname{Cel}(J)$, o que implica que as condições do teorema 1.4.7 são satisfeitas e portanto as equivalências fracas e fibrações dessa estrutura modelo cofibrantemente gerada são as mesmas do teorema 1.5.2, e como as cofibrações são determinadas pelas fibrações e equivalências fracas as cofibrações também coincidem.

\subsection{Categorias de Reedy generalizadas}

Precisaremos de estruturas modelo em categorias de diagramas em uma categoria modelo cofibrantemente gerada. Se a categoria que indexa os diagramas satisfaz certas condições existe uma estrutura modelo que nos permite fazer argumentos indutivos. Primeiro olhamos o caso particular de diagramas indexados por um grupo discreto.

Seja $G$ um grupo discreto (que podemos pensar como uma categoria pequena com um objeto e cujos morfismos são todos isomorfismos) e $\mathcal{T}$ uma categoria cocompleta. A categoria $\mathcal{T}^{G}$ é a categoria de objetos de $\mathcal{T}$ equipados com uma ação de $G$, i.e. a categoria de funtores $X: G \rightarrow \mathcal{T}$. Temos que o funtor esquecimento $U: \mathcal{T}^{G} \rightarrow \mathcal{T}$ admite um adjunto à esquerda

$$
\begin{aligned}
{[G]: \mathcal{T} } & \rightarrow \mathcal{T}^{G} \\
X & \mapsto \sqcup_{g \in G} X
\end{aligned}
$$

Teorema 1.6.1. Se $\mathcal{T}$ for uma categoria modelo cofibrantemente gerada e $G$ um grupo discreto então $\mathcal{T}^{G}$ admite uma estrutura modelo $\left(W^{G}, C^{G}, F^{G}\right)$ induzida da estrutura modelo $(W, C, F)$ de $\mathcal{T}$ pela adjunção $([G] \dashv U)$.

Demonstração: Temos que $\mathcal{T}^{G}$ é bicompleta pois o funtor esquecimento cria limites e colimites. A adjunção $([G] \dashv U)$ satisfaz as condições do teorema 1.4.7. A condição 1.4.7.i) é satisfeita pois $U$ preserva colimites filtrados. A condição 1.4.7.ii) é satisfeita pois cofibrações triviais são fechadas por coprodutos pela proposição 1.1.11.v). 
Uma categoria de Reedy generalizada é uma categoria com uma estrutura que permite definir diagramas e morfismos de diagramas sobre elas indutivamente. Essa estrutura também permite definir uma estrutura modelo na categoria de diagramas sobre as categorias de Reedy generalizadas [BM11].

Definição 1.6.2. Uma categoria de Reedy generalizada é uma categoria pequena $\mathcal{R}$ equipada com duas subcategorias abrangentes, i.e. contendo todos os objetos de $\mathcal{R}, \mathcal{R}-$ e $\mathcal{R}_{+}$e uma função $g r \in$ $\operatorname{Set}(o b \mathcal{R}, \mathbb{N})$, o grau dos objetos de $\mathcal{R}$, que satisfazem as seguintes condições:

i) Todo morfismo em $\mathcal{R}_{+}$que não é um isomorfismo aumenta o grau;

ii) Todo morfismo em $\mathcal{R}_{-}$que não é um isomorfismo diminui o grau;

iii) Todo isomorfismo em $\mathcal{R}$ preserva o grau;

iv) $\mathcal{R}_{-} \cap \mathcal{R}_{+}$é o subgrupoide maximal de $\mathcal{R}$;

v) Todo morfismo $r \in \mathcal{R}\left(R, R^{\prime}\right)$ admite uma fatoração $\left(r_{-}, r_{+}\right) \in$ Fat $(r)$ tal que $r_{-} \in \mathcal{R}_{-}$e $r_{+} \in \mathcal{R}_{+}$, e essa fatoração é única a menos de isomorfismo;

vi) Se $\sigma r=r$ para $\sigma \in \mathcal{R}_{-} \cap \mathcal{R}_{+}$e $r \in \mathcal{R}_{-}$então $\sigma$ é uma identidade.

Uma categoria de Reedy generalizada é dualizável se ela também satisfaz a seguinte condição:

vii) Se $r \sigma=r$ para $\sigma \in \mathcal{R}_{-} \cap \mathcal{R}_{+}$e $r \in \mathcal{R}_{+}$então $\sigma$ é uma identidade.

Temos diversos exemplos de categorias de Reedy generalizadas dualizáveis.

- A categoria $0 \rightarrow 1$ com o grau dado como indicado;

- A categoria $1 \leftarrow 0 \rightarrow 1^{\prime}$ com o grau dado como indicado;

- A categoria $0 \rightrightarrows 1$ com o grau dado como indicado;

- A categoria $\Delta$ de classes de isomorfismos de conjuntos finitos não-vazios linearmente ordenados $\langle m\rangle:=\{0<\cdots<m\}$ e funções que preservam ordem, com a função cardinalidade como o grau, $\Delta_{-}$as sobrejeções e $\Delta_{+}$as injeções;

- Todo grupoide pequeno $G \operatorname{com} G=G_{-}=G_{+}$;

- A categoria $\mathbb{S}_{\text {inj }}$ da sessão 4.1 que é um esqueleto da categoria de conjuntos finitos e injeções com grau dado pela cardinalidade, $\mathbb{S}_{\text {inj+ }}=\mathbb{S}_{\text {inj }}$ e $\mathbb{S}_{\text {inj- }}=\mathbb{S}$ a subcategoria de bijeções;

- A categoria $2 \mathbb{S}_{\text {inj }}$ da sessão 4.2 que é um esqueleto da categoria de 2-conjuntos finitos e 2injeções com grau dado pela cardinalidade, $2 \mathbb{S}_{\text {inj+ }}=2 \mathbb{S}_{\text {inj }}$ e $2 \mathbb{S}_{\text {inj- }}=2 \mathbb{S}$ a subcategoria de 2-bijeções; 
- A categoria oposta $\mathcal{R}^{\mathrm{op}}$ de uma categoria de Reedy generalizadas dualizável $\mathcal{R}$, com $\mathcal{R}_{-}^{\mathrm{op}}=$ $\mathcal{R}_{+}$e $\mathcal{R}_{+}^{\text {op }}=\mathcal{R}_{-}$.

Uma categoria de Reedy generalizada $\mathcal{R}$ admite uma filtração natural com $F^{\bullet} \mathcal{R}$ a subcategoria plena dos objetos com grau menor ou iguais a $\bullet \in \mathbb{N}$. Podemos usar essa filtração para definir e estudar objetos e morfismos em $\mathcal{T}^{\mathcal{R}}$ indutivamente. Note que como todos os morfismos em $F^{0} \mathcal{R}$ são isomorfismos um diagrama $X \in \mathcal{T}^{F^{0} \mathcal{R}}$ é simplesmente uma escolha para cada $R \in \mathcal{R}$ com $\operatorname{gr}(R)=0$ de um $\mathcal{R}_{R}:=\mathcal{R}(R, R)$-objeto $X^{R} \in \mathcal{T}^{\mathcal{R}_{R}}$.

Definição 1.6.3. Seja $\mathcal{T}$ uma categoria bicompleta, $\mathcal{R}$ uma categoria de Reedy generalizada e $X^{-} \in \mathcal{T}^{F^{q-1}} \mathcal{R}$.

Para cada $R \in \mathcal{R}$ com $\operatorname{gr}(R)=q$ defina $\mathcal{R}^{+}(R)$ como a subcategoria plena de $\mathcal{R}_{+/ R}$ contendo todos os morfismos $r \in \amalg_{Q \in \mathcal{R}_{+}} \mathcal{R}_{+}(Q, R)$ exceto os isomorfismos. Temos que $\mathcal{R}_{R}$ age sobre $\mathcal{R}^{+}(R)$ por composição. O objeto de fecho de $X^{-}$em $R$ é o objeto $L X^{R} \in \mathcal{T}$ dado por $L X^{R}:=\operatorname{colim}_{r \in \mathcal{R}^{+}(R)} X^{\operatorname{Dom} r}$. Note que $L X^{R}$ admite uma ação de $\mathcal{R}_{R}$.

Para cada $R \in \mathcal{R}$ com $\operatorname{gr}(R)=q$ defina $\mathcal{R}^{-}(R)$ como a subcategoria plena de $\mathcal{R}_{-}^{R /}$ contendo todos os morfismos $r \in \bigsqcup_{S \in \mathcal{R}_{-}} \mathcal{R}_{-}(R, S)$ exceto os isomorfismos. Temos que $\mathcal{R}_{R}$ age sobre $\mathcal{R}^{-}(R)$ por precomposição. O objeto correspondente de $X^{-}$em $R$ é o objeto $M X^{R} \in \mathcal{T}$ dado por $M X^{R}:=$ $\lim _{r \in \mathcal{R}^{-}(R)} X^{\operatorname{Codom} r}$. Note que $M X^{R}$ admite uma ação de $\mathcal{R}_{R}$.

Por construção para todo $X^{-} \in \mathcal{T}^{F^{q-1}} \mathcal{R}$ e $R \in \mathcal{R}$ com $\operatorname{gr}(R)=q$ existe um morfismo natural $k^{R} \in \mathcal{T}^{\mathcal{R}_{R}}\left(L X^{R}, M X^{R}\right)$. Uma extensão de $X^{-}$em $F^{q} \mathcal{R}$ é uma escolha para cada $R \in \mathcal{R}$ com $\operatorname{gr}(R)=q$ de uma fatoração $\left(l^{R}, m^{R}\right) \in \mathcal{T}^{\mathcal{R}_{R}}\left(L X^{R}, X^{R}\right) \times \mathcal{T}^{\mathcal{R}_{R}}\left(X^{R}, M X^{R}\right) \cap \mathrm{Fat}\left(k^{R}\right)$. Portanto podemos descrever indutivamente diagramas indexados por categorias de Reedy generalizadas via uma indução no grau dos objetos.

Da mesma forma podemos indutivamente definir morfismos entre diagramas. Suponha que temos $X^{-}, Y^{-} \in \mathcal{T}^{\mathcal{R}}$. Um morfismo $f^{-} \in \mathcal{T}^{F^{0} \mathcal{R}}\left(X^{-}, Y^{-}\right)$é simplesmente uma escolha de um morfismo

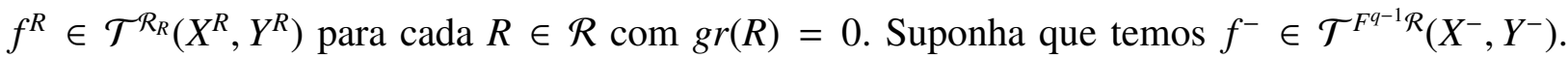
Uma extensão $f^{-} \in \mathcal{T}^{F^{q} \mathcal{R}}\left(X^{-}, Y^{-}\right)$de $f^{-}$é uma escolha $f^{R} \in \mathcal{T}^{\mathcal{R}_{R}}\left(X^{R}, Y^{R}\right)$ para cada $R \in \mathcal{R}$ com $\operatorname{gr}(R)=q$ tal que o seguinte diagrama em $\mathcal{T}^{\mathcal{R}_{R}}$ comuta:

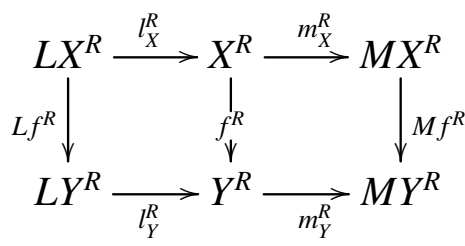


Suponha agora que temos a parte sólida do seguinte diagrama de objetos de $\mathcal{T}^{\mathcal{R}}$ :

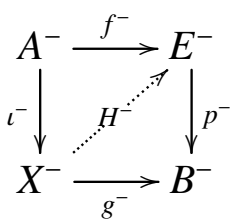

e suponha que existe um $H^{-} \in \mathcal{T}^{F^{q-1}} \mathcal{R}\left(X^{-}, E^{-}\right)$que faz a restrição do diagrama em $F^{q-1} \mathcal{R}$ comutar. Temos então que podemos estender $H^{-}$em $F^{q} \mathcal{R}$ se e somente se para cada $R \in \mathcal{R}$ com $\operatorname{gr}(R)=q$ existe $H^{R} \in \mathcal{T}^{\mathcal{R}_{R}}\left(X^{R}, E^{R}\right)$ que faz o seguinte diagrama comutar:

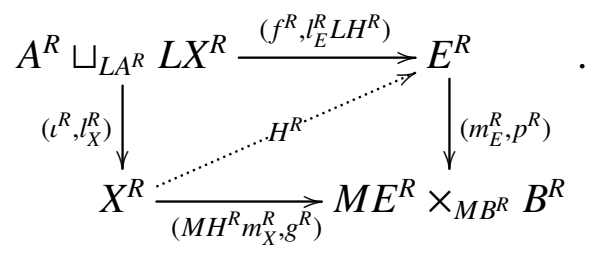

Isso motiva a seguinte definição.

Definição 1.6.4. Sejam $\mathcal{T}$ uma categoria modelo cofibrantemente gerada, $\mathcal{R}$ uma categoria de Reedy generalizada e $f^{-} \in \mathcal{T}^{\mathcal{R}}\left(X^{-}, Y^{-}\right)$.

A transformação natural $f^{-}$é uma equivalência fraca de Reedy se para todo $R \in \mathcal{R}$ temos $f^{R} \in W^{\mathcal{R}_{R}}\left(X^{R}, Y^{R}\right)$. Denotamos a classe de equivalências fracas de Reedy por $W^{\mathcal{R}}$.

A transformação natural $f^{-}$é uma cofibração de Reedy se para todo $R \in \mathcal{R}$ temos $\left(f^{R}, l_{Y}^{R}\right) \in$ $C^{\mathcal{R}_{R}}\left(X^{R} \sqcup_{L X^{R}} L Y^{R}, Y^{R}\right)$. Denotamos a classe de cofibrações de Reedy por $C^{\mathcal{R}}$.

A transformação natural $f^{-}$é uma fibração de Reedy se para todo $R \in \mathcal{R}$ temos $\left(m_{X}^{R}, f^{R}\right) \in$ $F^{\mathcal{R}_{R}}\left(X^{R}, M X^{R} \times_{M Y^{R}} Y^{R}\right)$. Denotamos a classe de fibrações de Reedy por $F^{\mathcal{R}}$.

Proposição 1.6.5. Sejam $\mathcal{T}$ uma categoria modelo cofibrantemente gerada, $\mathcal{R}$ uma categoria de Reedy e $f^{-} \in \mathcal{T}^{\mathcal{R}}\left(X^{-}, Y^{-}\right)$. Então $f^{-} \in C^{\mathcal{R}} \cap W^{\mathcal{R}}$ se e somente se para todo $R \in \mathcal{R}$ temos $\left(f^{R}, l_{Y}^{R}\right) \in$ $C^{\mathcal{R}} \cap W^{\mathcal{R}}\left(X^{R} \sqcup_{L X^{R}} L Y^{R}, Y^{R}\right)$, e $f^{-} \in F^{\mathcal{R}} \cap W^{\mathcal{R}}$ se e somente se para todo $R \in \mathcal{R}$ temos $\left(m_{X}^{R}, f^{R}\right) \in$ $F^{\mathcal{R}}\left(X^{R}, M X^{R} \times_{M Y^{R}} Y^{R}\right)$.

Demonstração: Provamos a primeira afirmação. A segunda é dual. Suponha que $f^{-} \in C^{\mathcal{R}} \cap$ $W^{\mathcal{R}}$. Pela proposição 1.1.11.iv) e a propriedade dois-de-três basta provar que para todo $R \in \mathcal{R}$ temos que $L f^{R} \in C \cap W$.

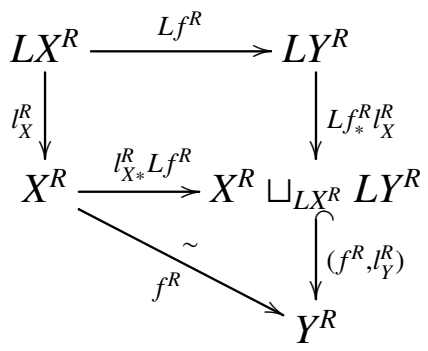


Se $\operatorname{gr}(R)=0$ então $L X^{R}=\emptyset=L Y^{R}$ e portanto trivialmente temos que $L f^{R} \in C \cap W$ e portanto que $\left(f^{R}, l_{Y}^{R}\right)=f^{R} \in C^{\mathcal{R}_{R}} \cap W^{\mathcal{R}_{R}}$. Provamos agora que supondo que $g r(R)=q$ e que para todo $Q \in \mathcal{R}$ com $g r(Q)<q$ temos que $\left(f^{Q}, l_{Y}^{Q}\right) \in C^{\mathcal{R}_{Q}} \cap W^{\mathcal{R}_{Q}}$ então $L f^{R} \in C \cap W$. Seja $p \in F(E, B)$ e $\left(\left(u_{r}\right)_{r \in \mathcal{R}^{+}(R)},\left(v_{r}\right)_{r \in \mathcal{R}^{+}(R)}\right) \in \mathcal{T}^{\rightarrow}\left(L f^{R}, p\right)$. Podemos construir um levantamento $\left(H_{r}\right)_{r \in \mathcal{R}^{+}(R)} \in \mathcal{T}\left(L Y^{R}, E\right)$ indutivamente no grau do domínio de $r \in \mathcal{R}^{+}(R)$. Se $g r(\operatorname{Dom}(r))=0$ então como vimos $f^{\operatorname{Dom}(r)} \in$ $C \cap W$ e portanto $H_{r}$ está bem definido. Para $r \in \mathcal{R}^{+}(R)$ com $0<\operatorname{gr}(\operatorname{Dom}(r))<q$ então temos $\left(\left(u_{r},\left(v_{s}\right)_{s \in \mathcal{R}^{+}(\operatorname{Dom}(r))}\right), v_{r}\right) \in \mathcal{T}^{\rightarrow}\left(\left(f^{\operatorname{Dom}(r)}, l_{Y}^{\operatorname{Dom}(r)}\right), p\right)$ e como por hipótese $\left(f^{\operatorname{Dom}(r)}, l_{Y}^{\operatorname{Dom}(r)}\right) \in C^{\mathcal{R}_{Q}} \cap$ $W^{\mathcal{R}_{Q}} \subset C \cap W$ existe um levantamento $H_{r} \in \mathcal{T}\left(Y_{\operatorname{Dom}(r)}, E\right)$. Temos portanto que $L f^{R} \in C \cap W$.

Suponha que para todo $R \in \mathcal{R}$ temos $\left(f^{R}, l_{Y}^{R}\right) \in C^{\mathcal{R}_{R}} \cap W^{\mathcal{R}_{R}}\left(X^{R} \sqcup_{L X^{R}} L Y^{R}, Y^{R}\right)$. Pelo argumento acima temos que $L f^{R} \in C \cap W$ para todo $R \in \mathcal{R}$ e portanto $f^{R} \in W^{\mathcal{R}_{R}}$

Teorema 1.6.6. Seja $\mathcal{T}$ uma categoria modelo cofibrantemente gerada e $\mathcal{R}$ uma categoria de Reedy generalizada. Existe uma estrutura modelo em $\mathcal{T}^{\mathcal{R}}$ em que as equivalências fracas são as equivalências fracas de Reedy $W^{\mathcal{R}}$, as cofibrações são as cofibrações de Reedy $C^{\mathcal{R}}$ e as fibrações são as fibrações de Reedy $F^{\mathcal{R}}$.

Demonstração: $\mathrm{O}$ funtor $a v: \mathcal{T}^{\mathcal{R}} \rightarrow \prod_{R \in \mathcal{R}} \mathcal{T}$ dado em cada coordenada $R \in \mathcal{R}$ pela avaliação do funtor no objeto $R$ cria limites e colimites, portanto $\mathcal{T}^{\mathcal{R}}$ é bicompleta.

Que $\left(\mathcal{T}^{\mathcal{R}}, W^{\mathcal{R}}\right)$ é uma categoria com equivalências fracas segue da estrutura de categoria com equivalências fracas de $\mathcal{T}$.

Que $C^{\mathcal{R}} \cap W^{\mathcal{R}}={ }^{\square} F^{\mathcal{R}}$ e $F^{\mathcal{R}} \cap W^{\mathcal{R}}=C^{\mathcal{R} \square}$ segue da proposição 1.6 .5 e dos comentários que motivaram a definição 1.6.4. Também podemos indutivamente definir os funtores e transformações naturais dos sistemas de fatorações fracos. Seja $f^{-} \in \mathcal{T}^{\mathcal{R}}\left(X^{-}, Y^{-}\right)$. Para $R \in \mathcal{R} \operatorname{com} \operatorname{gr}(R)=$

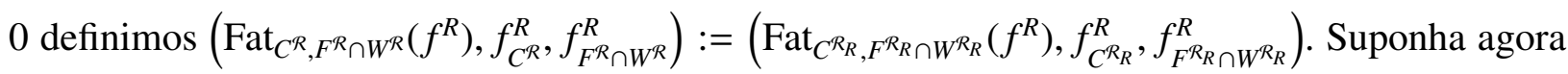
que a fatoração está bem definida em $F^{q-1} \mathcal{R}$. Temos que para todo $R \in \mathcal{R} \operatorname{com} \operatorname{gr}(R)=q \mathrm{o}$ morfismo

$$
\begin{gathered}
\tilde{f}^{R}:=\left(\left(f^{R}, M f_{C^{\mathcal{R}}}^{R} m_{X}^{R}\right),\left(l_{Y}^{R} L f_{F^{\mathcal{R}} \cap W^{\mathcal{R}}}^{R}, k_{\mathrm{Fat}_{C^{\mathcal{R}}, F^{\mathcal{R}} \cap W^{\mathcal{R}}}^{R}\left(f^{R}\right)}\right)\right) \in \\
\mathcal{T}^{\mathcal{R}_{R}}\left(X^{R} \sqcup_{L X^{R}} L \operatorname{Fat}_{C^{\mathcal{R}}, F^{\mathcal{R}} \cap W^{\mathcal{R}}}\left(f^{R}\right), Y^{R} \times_{M Y^{R}} M \operatorname{Fat}_{C^{\mathcal{R}}, F^{\mathcal{R}} \cap W^{\mathcal{R}}}\left(f^{R}\right)\right)
\end{gathered}
$$

está bem definido, e portanto podemos definir

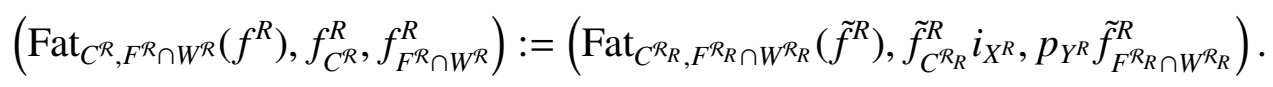

O outro sistema de fatoração fraco é definido analogamente. A proposição 1.6.5 garante que essas construções satisfazem as condições da definição 1.1.4. 


\subsection{Categorias modelo próprias}

As equivalências fracas em algumas categorias modelo são fechadas por pushouts ao longo de cofibrações e/ou pullbacks ao longo de fibrações. Essas propriedades permitem um maior controle das equivalências fracas. Em particular essa propriedade é necessária no teorema de localização de Bousfield da próxima sessão.

Definição 1.7.1. Seja $\mathcal{T}$ uma categoria modelo.

i) $\mathcal{T}$ é própria a esquerda se as equivalências fracas forem fechadas por pushouts ao longo de cofibração;

ii) $\mathcal{T}$ é própria a direita se as equivalências fracas forem fechadas por pullbacks ao longo de fibração;

iii) $\mathcal{T}$ é própria se for própria a esquerda e a direita.

Em toda categoria modelo equivalências fracas entre objetos cofibrantes (fibrantes) são preservados por pushouts ao longo de cofibrações (pullbacks ao longo de fibrações). Para mostrar isso precisamos do seguinte lema.

Lema 1.7.2. Seja $\mathcal{T}$ uma categoria modelo e $f \in \mathcal{T}(X, Y)$.

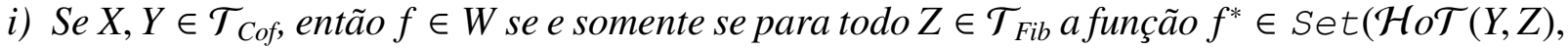
$\mathcal{H} o \mathcal{T}(X, Z))$ é um isomorfismo.

ii) Se $X, Y \in \mathcal{T}_{\text {Fib }}$, então $f \in W$ se e somente se para todo $A \in \mathcal{T}_{\text {Cof }}$ a função $f_{*} \in \operatorname{Set}(\mathcal{H} o \mathcal{T}(A, X)$, $\mathcal{H} o \mathcal{T}(A, Y))$ é um isomorfismo.

Demonstração: Provamos a primeira afirmação, já que a segunda é dual. Que se $f \in W$ e $Z \in \mathcal{T}_{\text {Fib }}$ então $f^{*}$ é um isomorfismo segue do lema 1.2.8, da proposição 1.2.6 e do lema 1.3.6.

Suponha agora que para todo $Z \in \mathcal{T}_{\text {Fib }}$ temos que $f^{*}$ é um isomorfismo. Pelo lema 1.2.8 temos que Fib $f^{*}$ também é um isomorfismo. Logo pela propriedade dois-de-três e pelo teorema de Whitehead 1.2.12 basta mostrar que Fibf é uma equivalência homotópica. Pelo isomorfismo Fib $f^{*} \in \operatorname{Set}(\mathcal{H} o \mathcal{T}($ Fib $Y$, Fib $X), \mathcal{H} o \mathcal{T}($ Fib $X$, Fib $X))$ temos que existe um $g \in \mathcal{T}($ Fib $Y$, Fib $X)$ tal que $g$ Fib $f \simeq 1_{\text {FibX }}$. Pelo teorema 1.2.11 e por $\operatorname{Fib} f^{*} \in \operatorname{Set}(\mathcal{H} o \mathcal{T}(\operatorname{Fib} Y, \operatorname{Fib} Y), \mathcal{H} o \mathcal{T}(\operatorname{Fib} X, \operatorname{Fib} Y))$ ser um isomorfismo temos que $g^{*} \in \operatorname{Set}(\mathcal{H} o \mathcal{T}(\operatorname{Fib} X, \operatorname{Fib} Y), \mathcal{H} o \mathcal{T}(\operatorname{Fib} Y$, FibY)) é um isomorfismo, e portanto existe um $h \in \mathcal{T}(\operatorname{Fib} X, \operatorname{Fib} Y)$ tal que $h g \simeq 1_{\mathrm{Fib} Y}$, e portanto $\operatorname{Fib} f g \simeq h g \operatorname{Fib} f g \simeq$ $h g \simeq 1_{Y}$. Logo Fib $f$ é uma equivalência homotópica.

Proposição 1.7.3. Seja $\mathcal{T}$ uma categoria modelo.

1. Sejam $A, B \in \mathcal{T}_{\text {Cof }}, f \in W(A, B) e \iota \in C(A, X)$. Então $\iota^{*} f \in W$; 
2. Sejam $A, B \in \mathcal{T}_{F i b}, f \in W(A, B)$ e $p \in F(E, B)$. Então $p_{*} f \in W$.

Demonstração: Provamos a primeira parte, já que a segunda é dual. Temos o quadrado de pushout

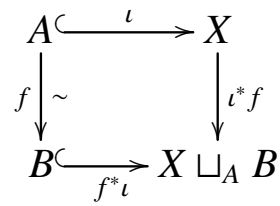

onde $f^{*} \iota \in C$ pela proposição 1.1.11.iv), e portanto $X, X \sqcup_{A} B \in \mathcal{T}_{\text {Cof }}$ pela proposição 1.1.11.ii). Logo pelo lema 1.7.2 basta provarmos que para todo $Z \in \mathcal{T}_{\text {Fib }}$ temos que $\iota^{*} f^{*} \in \operatorname{Set}\left(\mathcal{H} o \mathcal{T}\left(X \sqcup_{A}\right.\right.$ $B, Z), \mathcal{H} o \mathcal{T}(X, Z))$ é um isomorfismo.

Seja $k \in \mathcal{T}(X, Z)$. Pelo lema 1.7.2 aplicado em $f$ temos que existe $l \in \mathcal{T}(B, Z)$ tal que $l f \simeq k l$, ou seja existe uma homotopia $H \in \mathcal{T}(A, \operatorname{Cam}(Z))$ de $k \iota$ em $l f$. Como $\iota \in C$ e $Z \in \mathcal{T}_{\text {Fib }}$ pelo lema 1.2.2 temos um levantamento $\tilde{H} \in \mathcal{T}(X, \operatorname{Cam}(Z))$ de $(H, k) \in \mathcal{T} \rightarrow\left(\iota, p_{0}\right)$

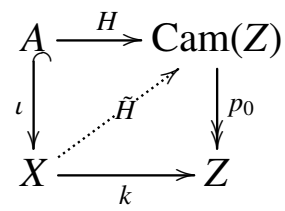

tal que $l f=p_{1} \tilde{H} \iota$ e $p_{0} \tilde{H}=k$. Temos então $\left(p_{1} \tilde{H}, l\right) \in \mathcal{T}\left(X \sqcup_{A} B, Z\right)$ tal que $\left(p_{1} \tilde{H}, l\right) \iota^{*} f \simeq k$. Logo $\iota^{*} f^{*}$ é sobrejetora.

Suponha agora que temos $(k, l),\left(k^{\prime}, l^{\prime}\right) \in \mathcal{T}\left(X \sqcup_{A} B, Z\right)$ tais que $(k, l) \iota^{*} f \simeq\left(k^{\prime}, l^{\prime}\right) \iota^{*} f$, ou seja temos $H \in \mathcal{T}(X, \operatorname{Cam}(Z))$ tal que $p_{0} H=k$ e $p_{1} H=k^{\prime}$.

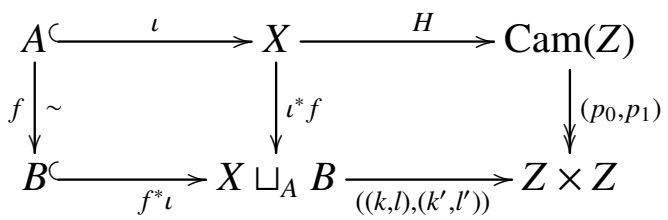

Temos que $\left(p_{0}, p_{1}\right) \in \mathcal{T}(\operatorname{Cam}(Z), Z \times Z)$ é um objeto fibrante de $\mathcal{T}_{\mid Z \times Z}$, e portanto pelo lema 1.7.2 existe $\tilde{H} \in \mathcal{T}(B, \operatorname{Cam}(Z))$ tal que $\tilde{H} f \simeq H \iota$ em $\mathcal{T}_{\mid Z \times Z}$, ou seja existe $K \in \mathcal{T}_{\text {/Z×Z }}(A, \operatorname{Cam}(\operatorname{Cam}(Z)))$ tal que $p_{0} K=\tilde{H} f$ e $p_{1} K=H \iota$. Como $\iota \in C$ e $\operatorname{Cam}(Z) \in \mathcal{T}_{\text {Fib }}$ pelo lema 1.2.2 temos um levantamento $\tilde{K} \in \mathcal{T}(X, \operatorname{Cam}(\operatorname{Cam}(Z)))$ de $(K, H) \in \mathcal{T}^{\rightarrow}\left(\iota, p_{0}\right)$

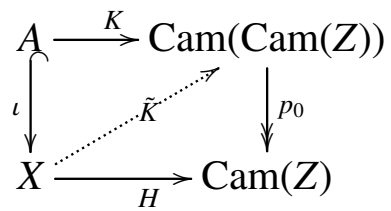

tal que temos $\tilde{H} f=p_{1} \tilde{K} \iota$, e também que $\left(p_{1} \tilde{K}, \tilde{H}\right) \in \mathcal{T}\left(X \sqcup_{A} B\right.$, Cam $\left.(Z)\right)$ é tal que $p_{0}\left(p_{1} \tilde{K}, \tilde{H}\right)=$ $(k, l)$ e $p_{1}\left(p_{1} \tilde{K}, \tilde{H}\right)=\left(k^{\prime}, l^{\prime}\right)$, portanto temos que $(k, l) \simeq\left(k^{\prime}, l^{\prime}\right)$. Logo $\iota^{*} f^{*}$ é injetora 


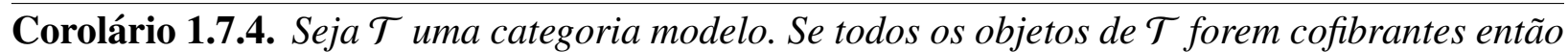
$\mathcal{T}$ é própria à esquerda. Se todos os objetos de $\mathcal{T}$ forem fibrantes então $\mathcal{T}$ é própria à direita.

Demonstração: Isso é uma consequência direta da proposição 1.7.3.

Proposição 1.7.5. Seja $\mathcal{T}$ uma categoria modelo cofibrantemente gerada própria à direita e $(S \dashv$ $\Lambda): \mathcal{T} \rightleftharpoons \mathcal{A}$ uma adjunção que satisfaz as condições do teorema 1.4.7. Então a estrutura modelo transferida em $\mathcal{A}$ é própria à direita.

Demonstração: Segue que adjuntos à direita preservam limites e que por definição $\Lambda$ preserva fibrações e equivalências fracas.

Proposição 1.7.6. Se $\mathcal{T}$ é uma categoria própria à esquerda (direita) e $Z \in \mathcal{T}$, então $\mathcal{T}^{Z /}$ é uma categoria modelo própria à esquerda (direita).

Demonstração: Isso segue do fato que o funtor esquecimento $U: \mathcal{T}^{Z /} \rightarrow \mathcal{T}$ preserva pushouts e pullbacks.

\subsection{Localização de Bousfield}

Definição 1.8.1. Seja $\mathcal{T}$ uma categoria modelo com estrutura modelo $(W, C, F)$. Uma localização de Bousfield à esquerda $\mathcal{T}_{L}$ de $\mathcal{T}$ é uma nova estrutura modelo $\left(W_{L}, C_{L}, F_{L}\right)$ em $\mathcal{T}$, i.e. $\mathcal{T}_{L}=\mathcal{T}$ como categorias, tal que $C=C_{L}$ e $W \subset W_{L}$.

Uma localização de Bousfield à direita $\mathcal{T}_{R}$ de $\mathcal{T}$ é uma nova estrutura modelo $\left(W_{R}, C_{R}, F_{R}\right)$ em $\mathcal{T}$, i.e. $\mathcal{T}_{R}=\mathcal{T}$ como categorias, tal que $F=F_{R}$ e $W \subset W_{R}$.

Nessa sessão exploramos explicitamente localizações de Bousfield à esquerda, mas todos os resultados e definições admitem dualizações para localizações de Bousfield à direita. Uma localização de Bousfield à esquerda pode ser pensada como uma versão homotópica de uma subcategoria reflexiva, i.e. uma subcategoria plena cuja inclusão admite um adjunto à esquerda, a reflexão dessa subcategoria.

Proposição 1.8.2. Dada uma localização de Bousfield à esquerda $\mathcal{T}_{L}$ de uma categoria modelo $\mathcal{T}$ então

i) $F_{L} \subset F$;

ii) $F_{L} \cap W_{L}=F \cap W$;

iii) O funtor identidade forma uma adjunção de Quillen $(I d \dashv I d): \mathcal{T} \rightleftharpoons \mathcal{T}_{L}$; 
iv) O funtor derivado à direita

$$
\begin{aligned}
\mathbb{R I d}: \mathcal{H}_{o} \mathcal{T}_{L} & \rightarrow \mathcal{H} o \mathcal{T} \\
X & \mapsto X \\
{[f]_{L} } & \mapsto[f]
\end{aligned}
$$

é a inclusão de uma subcategoria reflexiva com reflexão dada por $\mathbb{L} I d$

Demonstração: Pelas hipóteses temos $F_{L}=\left(C_{L} \cap W_{L}\right)^{\square} \subset(C \cap W)^{\square}=F$ e $F_{L} \cap W_{L}=$ $C_{L}^{\square}=C^{\square}=F \cap W$. Além disso claramente temos uma adjunção $(I d \dashv I d)$ e por construção $I d: \mathcal{T} \rightarrow \mathcal{T}_{L}$ preserva cofibrações e cofibrações triviais, logo essa é uma adjunção de Quillen. A última afirmação segue do ítem anterior e do fato que como $C=C_{L}$ e $F_{L} \subset F$ um objeto bifibrante em $\mathcal{T}_{L}$ é bifibrante em $\mathcal{T}$, e de que como $C=C_{L}$ e $F_{L} \cap W_{L}=F \cap W$ temos que a relação de homotopia à esquerda em $\mathcal{T}$ e em $\mathcal{T}_{L}$ coincidem.

Dada uma categoria $\mathcal{D}$ e uma subcategoria reflexiva $C$ temos que a reflexão $Q: \mathcal{D} \rightarrow C$ da inclusão, a unidade $\eta: I d_{\mathcal{D}} \Rightarrow Q$ e a counidade $\epsilon: Q \Rightarrow I d_{C}$ satisfazem as equações $1_{C}=$ $\epsilon_{C} \eta_{C}$ para $C \in C$ e $1_{Q D}=Q \epsilon_{D} \eta_{Q D}=\eta_{D} \epsilon_{D}$ para $D \in \mathcal{D}$. Portanto temos que a unidade $\eta$ é um isomorfismo nos objetos de $C$ e $\eta_{Q}$ é um isomorfismo em todos os objetos de $\mathcal{D}$. Por isso dizemos que o funtor $Q$ é idempotente.

Em [BF78, teo. A.7] Bousfield e Friedlander provam que em uma categoria modelo própria à direita um endofuntor que se comporta como uma reflexão em um sentido homotópico, que eles chamam de monada idempotente de Quillen, define uma localização de Bousfield à esquerda. Aqui, assim como na definição de uma quasi-adjunção de Quillen fraca, modificamos a definição original para levar em conta a necessidade da construção bar no teorema do reconhecimento e provamos que a demonstração dos teoremas relevantes seguem por argumentos análogos aos originais. Recuperamos a definição original se assumirmos que $=$ e $\eta^{\prime}$ são identidades.

Definição 1.8.3. Seja $\mathcal{T}$ uma categoria modelo própria à direita. Uma quasi-monada idempotente de Quillen é um par de endofuntores $Q: \mathcal{T} \rightarrow \mathcal{T}$ e $=: \mathcal{T} \rightarrow \mathcal{T}$ equipados com um par de transformações naturais $\eta^{\prime}:=\Rightarrow I d_{\mathcal{T}}$ e $\eta: \overline{=} Q Q$ tais que:

i) Para todo $X \in \mathcal{T}$ temos que $\eta_{X}^{\prime} \in W$;

ii) Para todo $f \in W$ temos que $Q f \in W$;

iii) Para todo $X \in \mathcal{T}$ temos que $Q \eta_{X}, \eta_{Q X} \in W$;

iv) Para todos $E, B, X \in \mathcal{T}, p \in F(E, B)$ e $f \in \mathcal{T}(X, B)$ tais que $\eta_{E}, \eta_{B}, Q f \in W$ temos que $Q\left(p_{E}\right) \in W\left(Q\left(X \times_{B} E\right), Q E\right)$.

v) Para todos $X, K \in \mathcal{T}$ e $\iota \in C(\bar{X}, K)$ temos que $i_{K} \in W\left(K, X \sqcup_{\bar{X}} K\right)$. 
Definição 1.8.4. Para $Q: \mathcal{T} \rightarrow \mathcal{T}$ uma quasi-monada idempotente de Quillen defina as classes de morfismos $W_{Q}:=Q^{-1}(W)$ as Q-equivalências fracas, $C_{Q}:=C$ as $Q$-cofibrações e $F_{Q}:=$ $\left(C_{Q} \cap W_{Q}\right)^{\square}$ as $Q$-fibrações.

Lema 1.8.5. Para $Q: \mathcal{T} \rightarrow \mathcal{T}$ uma quasi-monada idempotente de Quillen $W_{Q} \cap F_{Q}=W \cap F$.

Demonstração: Seja $f \in \mathcal{T}(X, Y)$. Suponha $f \in F \cap W$. Como $Q$ preserva equivalências fracas $f \in W_{Q}$. Como $C_{Q}=C$ temos que $f \in C_{Q}^{\square} \subset\left(C_{Q} \cap W_{Q}\right)^{\square}, \operatorname{logo} f \in F_{Q}$.

Suponha agora $f \in F_{Q} \cap W_{Q}$. Considere a fatoração $\left(f_{C}, f_{F \cap W}\right) \in \operatorname{Fat}(f)$. Como $Q$ preserva equivalências fracas e $W_{Q}$ satisfaz a propriedade dois-de-três temos que $f_{C} \in W_{Q}$ e portanto $f_{C} \in$ $C_{Q} \cap W_{Q}$. Logo $f_{C} \nabla f$ e pelo argumento da retração 1.1.8 temos $f \in \operatorname{Ret}\left(f_{F \cap W}\right)$, $\log$ o por 1.1.11.iii) temos que $f \in F \cap W$.

Lema 1.8.6. Seja $Q: \mathcal{T} \rightarrow \mathcal{T}$ uma quasi-monada idempotente de Quillen e $f \in F(X, Y)$. Se $\eta_{X}, \eta_{Y} \in W$ então $f \in F_{Q}$.

Demonstração: Suponha que temos um $\iota \in C_{Q} \cap W_{Q}(K, L)$ e um quadrado comutativo $(k, l) \in$ $\mathcal{T}^{\rightarrow}(\iota, f)$. Considere a seguinte fatoração de $Q(k, l)$ :

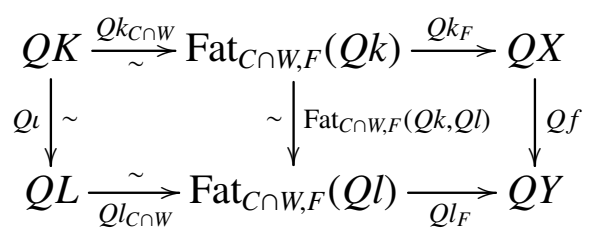

Por $Q$ preservar equivalências fracas e pela propriedade dois-de-três $\operatorname{Fat}_{C \cap W, F}(Q k, Q l)$ é uma equivalência fraca. Tomando o pullback do quadrado da direita por $\left(\eta_{X}, \eta_{Y}\right)$ obtemos o diagrama

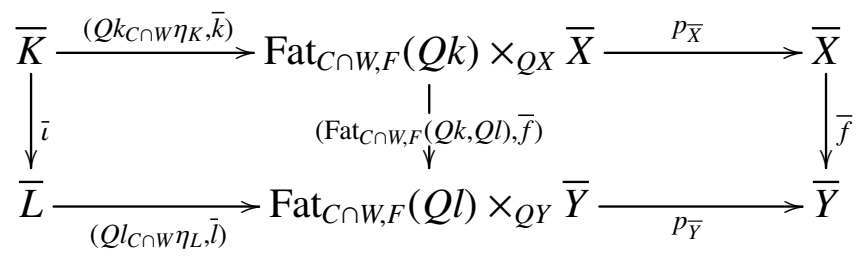

aonde $\left(\right.$ Fat $\left._{C \cap W, F}(Q k, Q l), \bar{f}\right)$ é uma equivalência fraca pela propriedade dois-de-três, $\eta_{X}, \eta_{Y} \in W, \mathcal{T}$ ser própria à direita e a existência do seguinte quadrado comutativo:

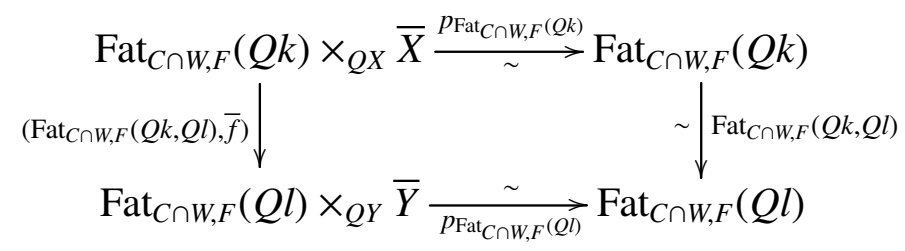


Tomando o pushout homotópico ${ }^{1}$ do quadrado da esquerda por $\left(\eta_{K}^{\prime}, \eta_{L}^{\prime}\right)$ obtemos

$$
\begin{array}{r}
K^{\prime}:=K \sqcup_{\bar{K}}^{H}\left(\operatorname{Fat}_{C \cap W, F}(Q k) \times_{Q X} \bar{X}\right) \\
L^{\prime}:=L \sqcup_{\bar{L}}^{H}\left(\operatorname{Fat}_{C \cap W, F}(Q l) \times_{Q Y} \bar{Y}\right) \\
\iota^{\prime}:=\left(\iota,\left(\operatorname{Fat}_{C \cap W, F}(Q k, Q l), \bar{f}\right)\right): K^{\prime} \rightarrow L^{\prime}
\end{array}
$$

tal que $\iota^{\prime}$ é uma equivalência fraca por ser um pushout homotópico de equivalências fracas. Temos então o seguinte diagrama:

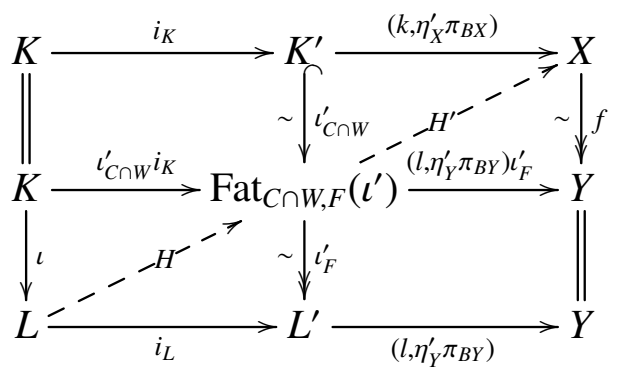

aonde $\iota_{F}^{\prime} \in W$ pela propriedade dois-de-três. Temos então que $H^{\prime} H \in \mathcal{T}(L, X)$ é um levantamento de $(k, l) \in \mathcal{T}^{\rightarrow}(\iota, f)$. Provamos assim que $f \in F_{Q}$

Teorema 1.8.7. Sejam $\mathcal{T}$ uma categoria modelo própria à direita e $Q: \mathcal{T} \rightarrow \mathcal{T}$ uma quasimonada idempotente de Quillen. Então $\left(\mathcal{T}_{Q}, W_{Q}, C_{Q}, F_{Q}\right)$ é uma localização de Bousfield à esquerda de $\mathcal{T}$ em $W_{Q}$.

Demonstração: Como $Q$ é um funtor ele preserva composições e isomorfismos, logo $W_{Q}$ contém todos os isomorfismos e satisfaz a propriedade dois-de-três, $\operatorname{logo}\left(\mathcal{T}, W_{Q}\right)$ é uma categoria com equivalências fracas. Pelo lema 1.8.5 temos que $\left(C_{Q}, F_{Q} \cap W_{Q}\right)=(C, F \cap W)$, e portanto definindo $\mathrm{Fat}_{C_{Q}, F_{Q} \cap W_{Q}}:=\mathrm{Fat}_{C, F \cap W}$ obtemos um sistema de fatoração fraco. Precisamos provar então que existe um funtor $\mathrm{Fat}_{C_{Q} \cap W_{Q}, F_{Q}}$ tal que $\left(C_{Q} \cap W_{Q}, F_{Q} ;\right.$ Fat $\left._{C_{Q} \cap W_{Q}, F_{Q}}\right)$ é um sistema de fatoração fraco.

Primeiro note que por definição $F_{Q}=\left(C_{Q} \cap W_{Q}\right)^{\square}$, e que ${ }^{\square} F_{Q}={ }^{\square}\left(\left(C_{Q} \cap W_{Q}\right)^{\square}\right) \supset W_{Q} \cap C_{Q}$. Logo se definirmos a fatoração $\mathrm{Fat}_{C_{Q} \cap W_{Q}, F_{Q}}$ então pelo argumento da retração 1.1.8, o fato que $C_{Q}=C$ e $W$ são fechados por retração pela proposição 1.1.11.iii) e $Q$ preservar retrações por ser um funtor obtemos ${ }^{\square} F_{Q} \subset W_{Q} \cap C_{Q}$.

Defina $Q^{\prime} X:=\operatorname{Fat}_{C, F \cap W}\left(\eta_{X}\right)$. Pela hipótese 1.8.3.v) de quasi-monadas idempotentes temos que $i_{Q^{\prime} X} \in W\left(Q^{\prime} X, X \sqcup_{\bar{X}} Q^{\prime} X\right)$. Logo por $=$ e $Q$ preservarem equivalências fracas e pela propriedade

\footnotetext{
${ }^{1} \mathrm{O}$ colimite homotópico de um diagrama de Reedy, como por exemplo o diagrama de pushouts, pode ser computado tomando uma resolução cofibrante na estrutura modelo de Reedy e então tomando o colimite usual. O colimite homotópico, ao contrário do colimite usual, preserva equivalências fracas. Para detalhes da teoria de (Co)limites homotópicos veja [Hi09, GJ09].
} 
dois-de-três o diagrama abaixo nos dá que $\eta_{X \sqcup_{\bar{X}} Q^{\prime} X}$ é uma equivalência fraca para todo $X \in \mathcal{T}$.

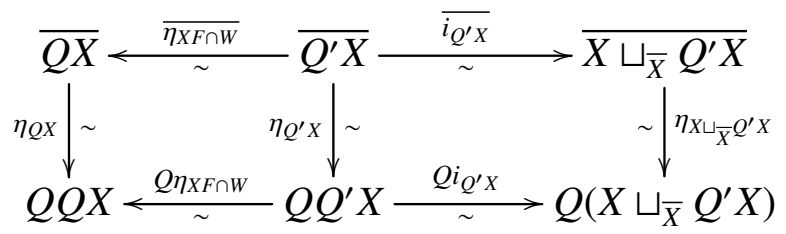

Considerando agora um morfismo $f \in \mathcal{T}(X, Y)$ qualquer podemos aplicar a fatoração Fat $_{C \cap W, F}$ em $\left(f, Q^{\prime} f\right)$ e o span natural de $Q$ para obter o seguinte diagrama:

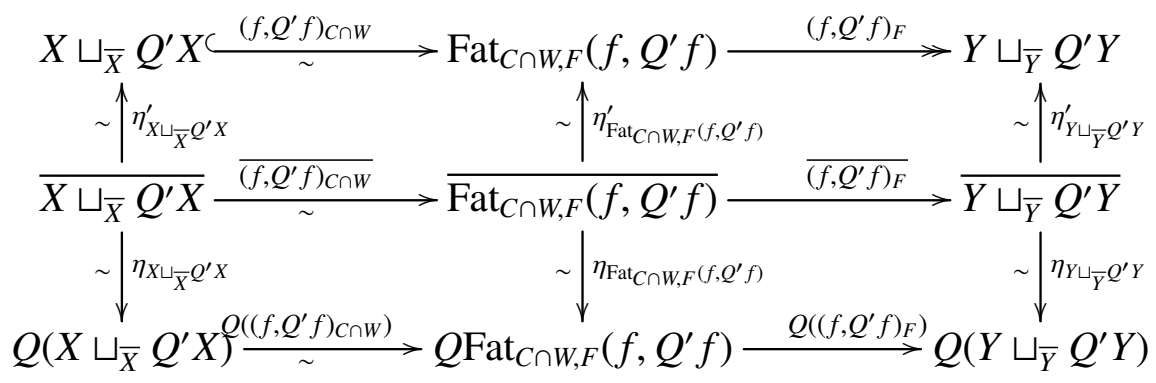

Pela observação anterior temos $\eta_{X \sqcup_{\bar{X}} Q^{\prime} X}, \eta_{Y \sqcup_{\bar{Y}} Q^{\prime} Y} \in W$, e pela propriedade dois-de-três $\eta_{\text {Fat }{ }_{C \cap W, F}\left(f, Q^{\prime} f\right)} \in$ $W$, logo pelo lema 1.8.6 temos $\left(f, Q^{\prime} f\right)_{F} \in F_{Q}$. Como $C=C_{Q}$ e $W \subset W_{Q}$ temos $\left(f, Q^{\prime} f\right)_{C \cap W} \in$ $C_{Q} \cap W_{Q}$.

Pela hipótese 1.8.3.iii) de quasi-monadas idempotentes e pela propriedade dois-de-três $Q \eta_{X C} \in$ $W$. Portanto pela propriedade dois-de-três $Q i_{X} \in W$, e portanto $i_{X} \in W_{Q}$ para todo $X \in \mathcal{T}$.

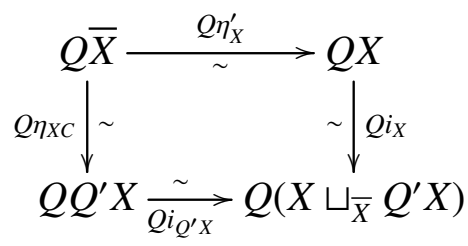

Portanto tomando o pullback de $\left(f, Q^{\prime} f\right)_{F}$ por $i_{Y}$ temos pela hipótese 1.8.3.iv) de quasi-monadas idempotentes que $\left(f, Q^{\prime} f\right)_{F *} i_{Y} \in W_{Q}$, que pela propriedade dois-de-três implica que $\left(\left(f, Q^{\prime} f\right)_{C \cap W} i_{X}\right.$, $f) \in W_{Q}$ e portanto no diagrama abaixo $\left(\left(f, Q^{\prime} f\right)_{C \cap W} i_{X}, f\right)_{C} \in C_{Q} \cap W_{Q}$. Como $F_{Q}$ é definido por uma propriedade de levantamento à direita a proposição $1.1 .11 \mathrm{iv})$ nos dá que $i_{Y *}\left(f, Q^{\prime} f\right)_{F} \in F_{Q}$, e portanto como $F \cap W=F_{Q} \cap W_{Q}$ pelo lema 1.8.5 temos que $i_{Y *}\left(f, Q^{\prime} f\right)_{F}\left(\left(f, Q^{\prime} f\right)_{C \cap W} i_{X}, f\right)_{F \cap W} \in$ $F_{Q}$.

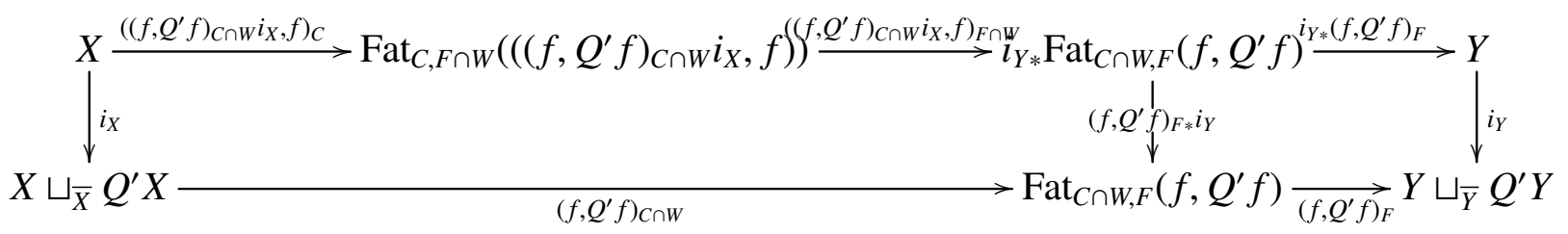

Logo os morfismos horizontais de cima do diagrama nos dá uma fatoração funtorial $\mathrm{Fat}_{C_{Q} \cap W_{Q}, F_{Q}}$ 
Lembrando da última demonstração que $Q^{\prime} X:=$ Fat $_{C, F \cap W}\left(\eta_{X}\right)$ e $\left(f, Q^{\prime} f\right) \in \mathcal{T}\left(X \sqcup_{\bar{X}} Q^{\prime} X, Y \sqcup_{\bar{Y}}\right.$ $\left.Q^{\prime} Y\right)$ denotamos $Q^{\prime \prime} X:=X \sqcup_{\bar{X}} Q^{\prime} X$ e temos a seguinte caracterização das $Q$-fibrações. Usamos resultados de pullbacks homotópicos de [GJ09, S. II.9], que são dados no contexto de conjuntos simpliciais mas valem para qualquer categoria própria à direita.

Proposição 1.8.8. Sejam $\mathcal{T}$ uma categoria modelo própria à direita, $Q: \mathcal{T} \rightarrow \mathcal{T}$ uma quasimonada idempotente de Quillen e $f \in \mathcal{T}(X, Y)$. Então $f \in F_{Q}$ se e somente se as seguintes condições forem satisfeitas:

i) $f \in F$;

ii) $O$ quadrado comutativo $\left(i_{X}, i_{Y}\right) \in \mathcal{T}^{\rightarrow}\left(f,\left(f, Q^{\prime} f\right)\right)$ é um pullback homotópico. Por $\mathcal{T}$ ser própria à direita essa condição é equivalente a que para todo $\left(\left(f, Q^{\prime} f\right)_{W},\left(f, Q^{\prime} f\right)_{F}\right)$ $\in W\left(Q^{\prime \prime} X, Z\right) \times F\left(Z, Q^{\prime \prime} Y\right) \cap F a t\left(\left(f, Q^{\prime} f\right)\right)$ temos que $f^{\prime} \in W\left(X, Y \times_{Q^{\prime \prime} Y} Z\right)$, sendo $f^{\prime}$ a aplicação induzida pela propriedade universal do pullback.

Demonstração: Suponha que $f$ satisfaz as condições $i)$ e $i i)$. Temos a fatoração $\left(\left(f, Q^{\prime} f\right)_{C \cap W}\right.$, $\left.\left(f, Q^{\prime} f\right)_{F}\right) \in \operatorname{Fat}\left(\left(f, Q^{\prime} f\right)\right)$, e pela demonstração do teorema anterior temos que $\left(f, Q^{\prime} f\right)_{F} \in F_{Q}$. Pela proposição 1.1.11.iv) temos que $p_{Y} \in F_{Q}\left(Y \times_{Y \sqcup_{\bar{Y}} Q^{\prime} Y} \operatorname{Fat}_{C \cap W, F}\left(\left(f, Q^{\prime} f\right)\right), Y\right)$.

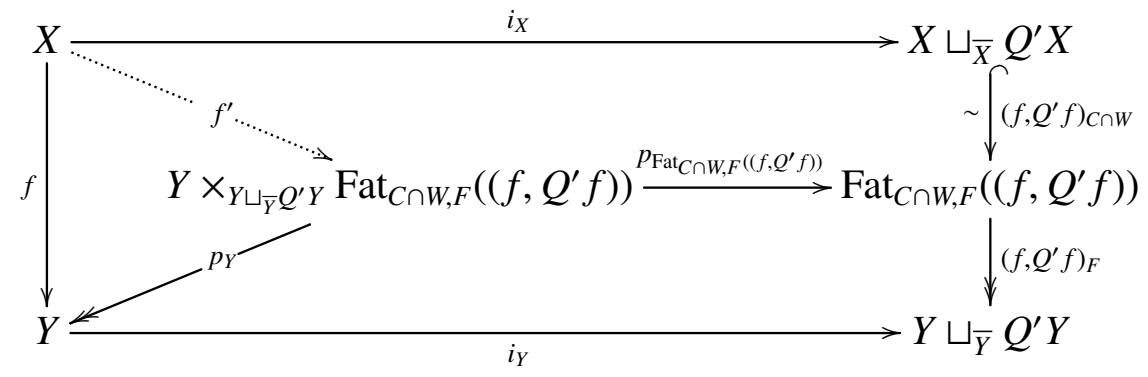

Temos a fatoração $\left(f_{C}^{\prime}, p_{Y} f_{F \cap W}^{\prime}\right) \in \operatorname{Fat}(f)$ e pela hipótese que $f^{\prime} \in W$ e a propriedade doisde-três temos que $f_{C}^{\prime} \in C \cap W$, e portanto pela hipótese que $f \in F$ temos que $f_{C}^{\prime} \square f$. Logo pelo argumento da retração 1.1 .8 temos que $f \in \operatorname{Ret}\left(p_{Y} f_{F \cap W}^{\prime}\right)$ e como $F \cap W=F_{Q} \cap W_{Q}$ o teorema 1.1.11.iii) nos dá que $f \in F_{Q}$.

Suponha agora que $f \in F_{Q}$. Como $F_{Q} \subset F$ a condição $\left.i\right)$ é satisfeita. Seja $\left.\left(\left(f, Q^{\prime} f\right)_{W},\left(f, Q^{\prime} f\right)_{F}\right)\right)$ como na condição $i$ ). Temos um span natural de equivalências fracas $Q^{\prime \prime} X \stackrel{\sim}{\leftarrow} Q^{\prime} X \stackrel{\sim}{\rightarrow} Q X$ pela definição de $Q^{\prime}$ e pela condição 1.8.3.v), e portanto pela propriedade dois-de-três temos que $Q^{\prime \prime} W_{Q} \subset W$. Considere o seguinte diagrama comutativo:

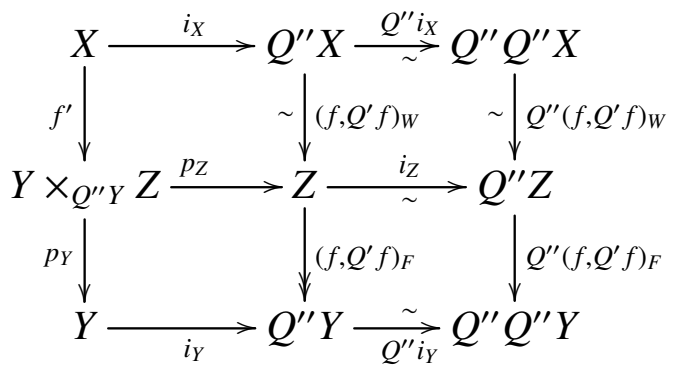


Pela demonstração do teorema anterior temos que $i_{X}, i_{Y} \in W_{Q}$ e $\left(f, Q^{\prime} f\right)_{F} \in F_{Q}$. Pela propriedade dois-de-três temos que $i_{Z} \in W$, e pela naturalidade de $\eta$ também temos pela propriedade doisde-três que $\eta_{Z} \in W$. Logo pela hipótese 1.8.3.iv) que $p_{Z} \in W_{Q}$. Também temos pela proposição 1.1.11.iv) que $p_{Y} \in F_{Q}$. Assim a propriedade dois-de-três de $W_{Q}$ implica que $f^{\prime} \in W_{Q}$. Como todo quadrado comutativo com morfismos paralelos que são equivalências homotópicas são pullbacks homotópicos e pullbacks em que um dos morfismos é uma fibração também são pullbacks homotópicos os dois quadrados de baixo no diagrama são pullbacks homotópicos, e portanto o retângulo inferior é um pullback homotópico. Esse retângulo inferior é igual ao retângulo

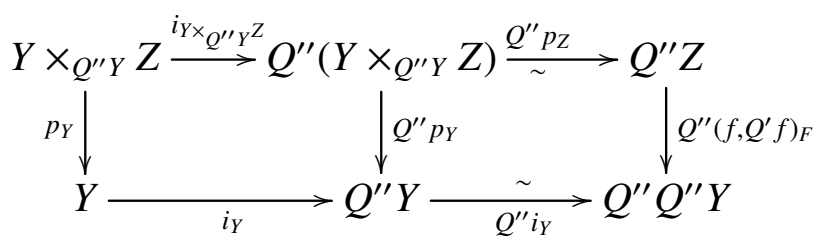

e como o quadrado da direita tem duas equivalências fracas paralelas ele é um pullback homotópico e portanto o quadrado da esquerda também é um pullback homotópico.

Temos a fatoração $\left(f_{C}^{\prime}, f_{F \cap W}^{\prime}\right) \in \operatorname{Fat}\left(f^{\prime}\right)$. Como $f^{\prime} \in W_{Q}$ e $F \cap W=F_{Q} \cap W_{Q}$ temos pela propriedade dois-de-três que $f_{C}^{\prime} \in C_{Q} \cap W_{Q}$. Logo $f_{C}^{\prime} \nabla f$ e pelo argumento da retração 1.1.8 temos que $f \in \operatorname{Ret}\left(p_{Y} f_{F \cap W}^{\prime}\right)$.

Temos então que o quadrado comutativo da condição $i$ ) é uma retração de

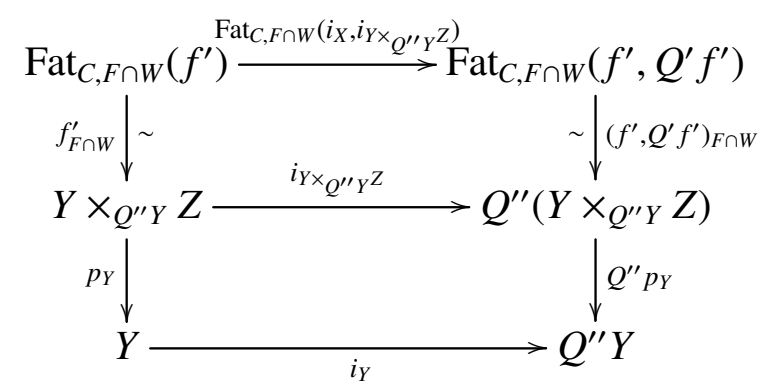

sendo que o quadrado de cima é um pullback homotópico por conter um par paralelo de equivalências fracas, e portanto o retângulo comutativo inteiro é um pullback homotópico. Assim como retrações preservam pullbacks homotópicos temos que a condição ii) é satisfeita.

Proposição 1.8.9. Seja $\mathcal{T}$ uma categoria própria à esquerda $e \mathcal{T}_{L}$ uma localização de Bousfield à esquerda de $\mathcal{T}$. Então $\mathcal{T}_{L}$ é própria à esquerda.

Demonstração: Seja $f \in W_{L}$. Temos $\left(f_{C}, f_{F \cap W}\right) \in \operatorname{Fat}(f)$. Como $W \subset W_{L}$ temos que $f_{F \cap W} \in$ $W_{L}$ e portanto pela propriedade dois-de-três $f_{C} \in C_{L} \cap W_{L}$. Logo que $W_{L}$ é preservado por pushouts por cofibrações segue da proposição 1.1.11.iv) e de $\mathcal{T}$ ser própria à esquerda. 


\subsection{Quasi-adjunções idempotentes de Quillen}

Dada uma quasi-adjunção de Quillen fraca $\left(S \dashv_{B, \bar{B}} \Lambda\right): \mathcal{T} \rightleftharpoons \mathcal{A}$ temos associado um endofuntor $\Lambda$ Fib $S$ Cof que preserva equivalências fracas equipado com um span natural

$$
I d_{\mathcal{T}} \stackrel{\eta^{\prime}}{\Longleftarrow} B \operatorname{Cof} \stackrel{\eta}{\rightleftharpoons} \Lambda \mathrm{FibS} \operatorname{Cof}
$$

e um endofuntor $S$ Cof $\Lambda$ Fib que preserva equivalências fracas equipado com um cospan

$$
S \operatorname{Cof} \Lambda \mathrm{Fib} \stackrel{\epsilon}{\Longrightarrow} \bar{B} \mathrm{Fib} \underset{\sim}{\stackrel{\epsilon^{\prime}}{\rightleftharpoons}} Y .
$$

Definição 1.9.1. Seja $\left(S \dashv_{B, \bar{B}} \Lambda\right): \mathcal{T} \rightleftharpoons \mathcal{A}$ uma quasi-adjunção de Quillen fraca com $\mathcal{T}$ própria à direita e $\mathcal{A}$ própria à esquerda. Se os endofuntores e as transformações naturais associadas formarem uma quasi-monada idempotente de Quillen e uma quasi-comonada idempotente de Quillen então dizemos que $\left(S \dashv_{B, \bar{B}} \Lambda\right)$ é uma quasi-adjunção idempotente de Quillen.

O teorema a seguir é uma versão homotópica do fato que se numa adjunção $(S \dashv \Lambda)$ temos que $\Lambda S$ é uma reflexão e $S \Lambda$ uma coreflexão então a adjunção induz uma equivalência entre uma sub-categoria reflexiva e uma sub-categoria coreflexiva. $\mathrm{O}$ teorema de reconhecimento de espaços de laços relativos infinitos 5.4.7 é um caso particular do seguinte teorema.

Teorema 1.9.2. Seja $\left(S \dashv_{B, \bar{B}} \Lambda\right): \mathcal{T} \rightleftharpoons \mathcal{A}$ com $\mathcal{T}$ própria à direita e $\mathcal{A}$ própria à esquerda uma quasi-adjunção idempotente de Quillen fraca. Então a adjunção $(\mathbb{L} S \dashv \mathbb{R} \Lambda)$ pode ser fatorada em uma equivalência entre a subcategoria reflexiva $\mathcal{H}_{o} \mathcal{T}_{\Lambda F i b S \text { Cof }}$ e a subcategoria coreflexiva $\mathcal{H}_{o} \mathcal{A}_{S \operatorname{Cof} \Lambda \text { Fib }}$.

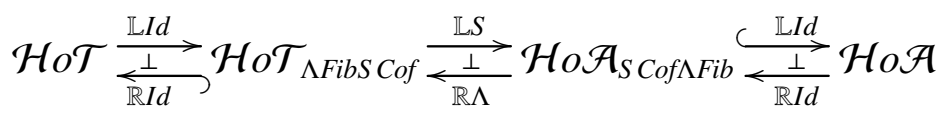

Demonstração: Pelas definições $\left(\Lambda \mathrm{fib}_{S} \eta\right)_{X}$ é uma $\Lambda$ FibS Cof-equivalência fraca e $\left(\epsilon S \operatorname{cof}_{\Lambda}\right)_{Y}$ é uma $S$ Cof $\Lambda$ Fib-equivalência fraca. Logo o teorema 1.3.10 nos dá a equivalência de categorias. 


\section{Capítulo 2}

\section{Categorias monoidais fechadas}

Apresentamos nesse capítulo a teoria de categorias modelo monoidais simétricas [HoM98], que é a estrutura subjacente de uma categoria que nos permite definir operads e 2-operads e em certas condições estruturas modelo nas categorias desses objetos. A propriedade básica dos operads e 2-operads definidos no capítulo 4 é que eles definem monadas na categoria pontuada da categoria ambiente. Apresentamos as definições de monadas, suas álgebras e funtores de monadas e descrevemos a construção bar bilateral.

Na primeira sessão apresentamos a definição de uma categoria monoidal e o que significa essa estrutura ser simétrica e fechada. A estrutura monoidal é um produto tensorial dada por um bifuntor que é associativo e unitário a menos de isomorfismos naturais. A estrutura modelo é simétrica se também for comutativa a menos de um isomorfismo natural idempotente. Uma estrutura monoidal é cartesiana se for dada pelo produto categorial, que é sempre simétrico e tem o objeto terminal como unidade. Finalmente uma categoria monoidal é fechada se para cada objeto o produto tensorial com esse objeto admitir um adjunto a direita, um funtor de objetos de morfismos interno à categoria. Essa estrutura extra garante que o produto tensorial com um objeto preserva colimites. A categoria de objetos pontuados de uma categoria monoidal fechada também herda uma estrutura monoidal fechada. Essa estrutura em $\mathrm{Top}_{*}$ nos permite definir espaços de laços e garante que essa construção é um adjunto à direita. Também apresentamos a estrutura monoidal fechada induzida na categoria de morfismos.

Na segunda sessão definimos categorias modelo monoidais, que é uma categoria bicompleta com uma estrutura modelo e uma estrutura monoidal fechada compatíveis. Apresentamos algumas propriedades de categorias modelo monoidais. O exemplo principal de categoria monoidal fechada nessa tese é a categoria Top de espaços compactamente gerados e fracamente Hausdorff do capítulo 3. Também apresentamos a definição de objetos intervalos de Hopf, cuja existência é uma das condições do teorema de existência de estruturas modelo na categoria de operads em [BM03, BM07].

Na terceira sessão damos a definição de monadas (também conhecidas como triplas), que são endofuntores em uma categoria equipados com estruturas de monoide que nos permitem inter- 
pretar as suas imagens como objetos livres de uma teoria algébrica [BW85]. Uma monada define álgebras, que são os objetos de sua teoria algébrica. Também damos a definição de funtores de monadas. Descrevemos como adjunções nos dão exemplos de monadas e funtores de monadas e damos algumas propriedades básicas. O conceito de monadas é central na demonstração do princípio do reconhecimento em [Ma72]. Também apresentamos a construção bar bilateral introduzida em [Ma72] que nos dá um objeto simplicial natural a partir de uma monada, uma álgebra e um funtor da monada. Usaremos essa construção para definir os espaços e aplicações necessários para provar o teorema de reconhecimento.

\subsection{Estrutura monoidal}

Uma estrutura monoidal em uma categoria nos permite definir a noção de um monoide interno da categoria, que é uma generalização da noção de um monoide da teoria de álgebra abstrata.

Definição 2.1.1. Uma categoria monoidal é uma categoria $\mathcal{T}$ equipada com um bifuntor $\otimes: \mathcal{T} \times$ $\mathcal{T} \rightarrow \mathcal{T}$, o produto tensorial de $\mathcal{T}$, um objeto $\mathbb{1} \in \mathcal{T}$, a unidade de $\mathcal{T}$, e uma tripla de isomorfismos naturais $\alpha_{X, Y, Z}:(X \otimes Y) \otimes Z \Rightarrow X \otimes(Y \otimes Z)$, o associador de $\mathcal{T}, \lambda_{X}: \mathbb{1} \otimes X \Rightarrow X$, o unidador à esquerda de $\mathcal{T}$, e $\rho_{X}: X \otimes \mathbb{1} \Rightarrow X$, o unidador à direita de $\mathcal{T}$, satisfazendo a condição de coerência que os diagramas abaixo comutam:

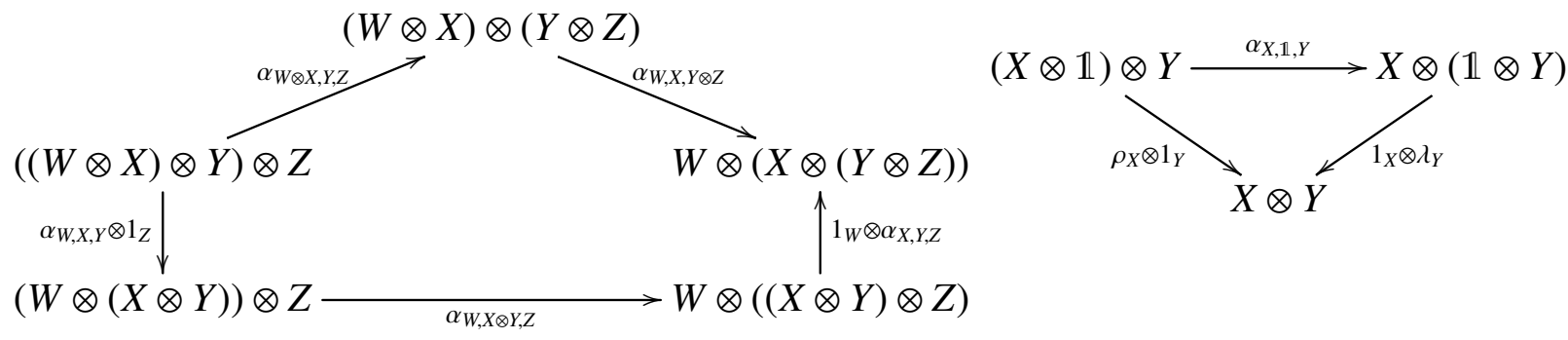

Uma categoria monoidal é cartesiana se o produto tensorial é dado pelo produto categorial $\times$. Nesse caso a unidade $\mathbb{1}$ deve ser um objeto terminal $*$.

Definição 2.1.2. Sejam $\mathcal{T}$ e $\mathcal{A}$ categorias monoidais. Um funtor monoidal é um funtor $F: \mathcal{T} \rightarrow \mathcal{A}$ equipado com um morfismo $\epsilon \in \mathcal{A}\left(\mathbb{1}_{\mathcal{A}}, F\left(\mathbb{1}_{\mathcal{T}}\right)\right)$ e uma transformação natural $v_{X, Y}: F X \otimes_{\mathcal{A}} F Y \Rightarrow$ $F\left(X \otimes_{\mathcal{T}} Y\right)$ satisfazendo a condição de coerência que os seguintes diagramas comutam:

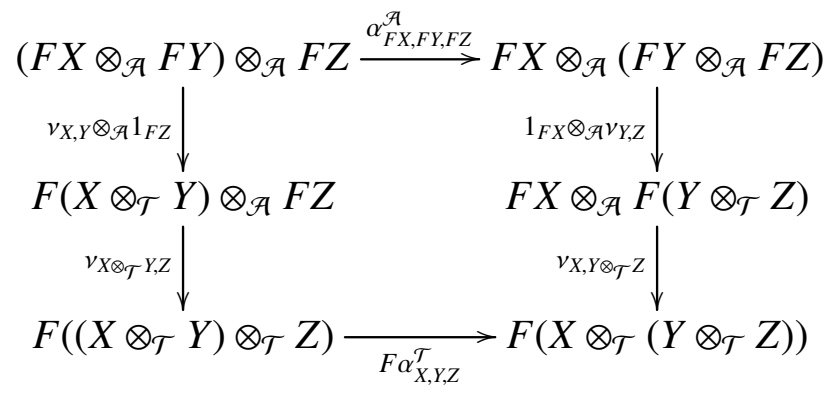



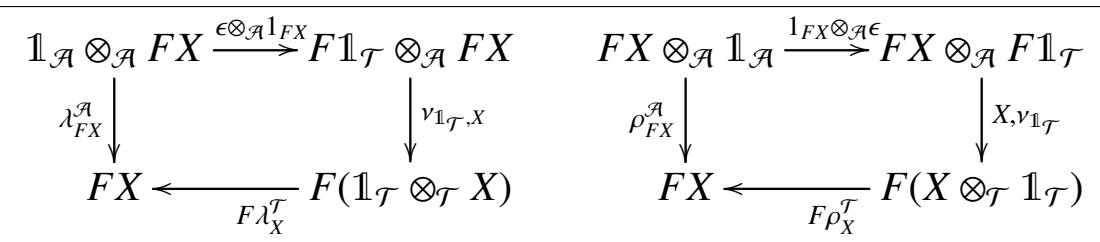

Definição 2.1.3. Um monoide em uma categoria monoidal $\mathcal{T}$ é um objeto $M \in \mathcal{T}$ equipado com um par de morfismos $\mu \in \mathcal{T}(M \otimes M, M)$, a multiplicação, e $\eta \in \mathcal{T}(\mathbb{1}, M)$, a unidade, satisfazendo a condição que os diagramas abaixo comutam:
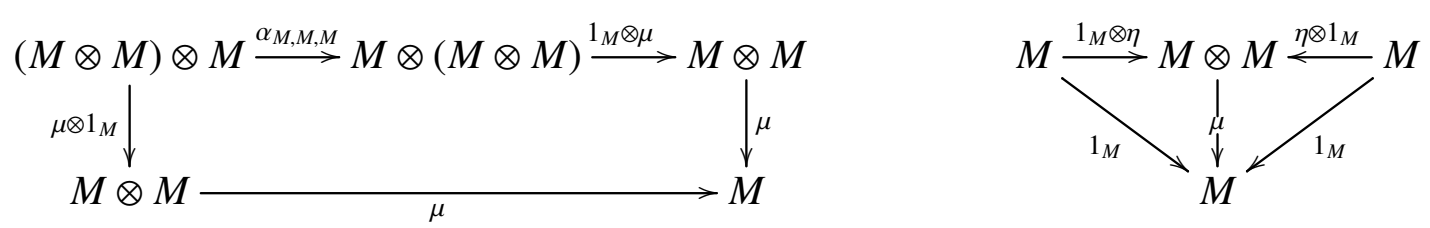

Um morfismo de monoides é um morfismo entre os objetos subjacentes que comutam com os diagramas estruturais.

Um comonoide é dualmente definido invertendo os morfismos na definição de um monoide, i.e. é um objeto $C \in \mathcal{T}$ equipado com um par de morfismos $\Delta: C \rightarrow C \otimes C$, a comultiplicação, e $\epsilon: C \rightarrow \mathbb{1}$, a counidade, satisfazendo a condição que os diagramas duais aos da definição de monoides comutam.

Definição 2.1.4. Uma categoria monoidal é simétrica se for equipada também com um isomorfismo natural $\tau_{X, Y}: X \otimes Y \Rightarrow Y \otimes X$ tal que $\tau_{Y \otimes X} \tau_{X \otimes Y}=1_{X \otimes Y}$ satisfazendo a condição de coerência que o diagrama abaixo comuta

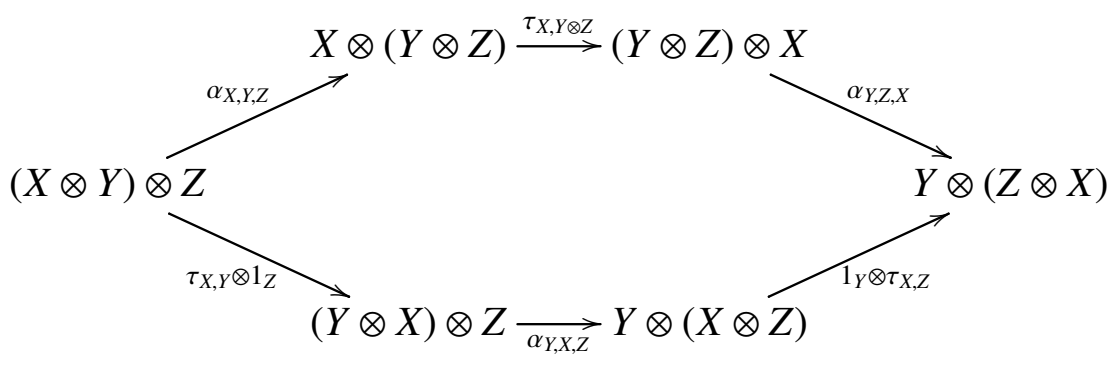

Uma categoria cartesiana é sempre simétrica.

Definição 2.1.5. Um monoide $M$ em uma categoria monoidal simétrica $\mathcal{T}$ é comutativo se satisfaz a condição que o diagrama abaixo comuta:

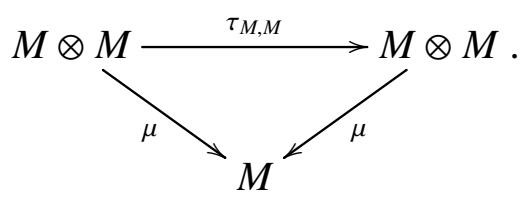

Definição 2.1.6. Sejam $\mathcal{T}$ e $\mathcal{A}$ categorias monoidais simétricas. Um funtor monoidal simétrico é um funtor monoidal $F: \mathcal{T} \rightarrow \mathcal{A}$ que satisfaz também a condição de coerência que o seguinte 
diagrama comuta:

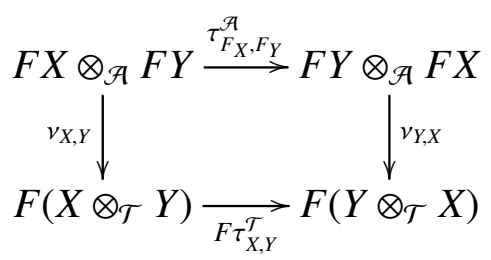

Definição 2.1.7. Uma categoria monoidal é fechada se for equipada também com um bifuntor $[-,-]: \mathcal{T}^{\mathrm{op}} \times \mathcal{T} \rightarrow \mathcal{T}$, o Hom interno, tal que para todo objeto $X \in \mathcal{T}$ temos uma adjunção $(-\otimes X \dashv[X,-])$. Denotamos a unidade dessa adjunção por $\eta^{X}$ e a counidade por $\epsilon^{X}$.

Proposição 2.1.8. Para todo objeto $X$ de uma categoria monoidal fechada $\mathcal{T}$ o funtor $-\otimes X$ preserva colimites. Se $\mathcal{T}$ for simétrica então $X \otimes-$ também preserva colimites.

Demonstração: Segue do fato que adjuntos à esquerda preservam colimites e da simetria ser dada por um isomorfismo natural

Se uma categoria monoidal fechada for bicompleta a sua categoria pontuada também herda uma estrutura monoidal fechada.

Definição 2.1.9. Seja $\mathcal{T}$ uma categoria monoidal fechada bicompleta. O produto smash em $\mathcal{T}_{*}$ é o bifuntor $\wedge: \mathcal{T}_{*} \times \mathcal{T}_{*} \rightarrow \mathcal{T}_{*}$, com $X \wedge Y$ definido como o pushout em $\mathcal{T}$ :

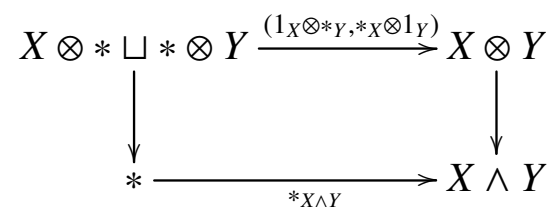

e o Hom interno pontuado em $\mathcal{T}_{*}$ é o bifuntor $[-,-]_{*}: \mathcal{T}_{*}^{\mathrm{op}} \times \mathcal{T}_{*}$, com $[X, Y]_{*}$ definido como o pullback em $\mathcal{T}$ :

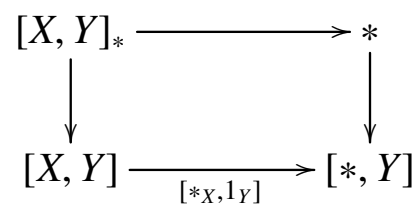

aonde $* \rightarrow[*, Y]$ é o morfismo adjunto da composição $* \otimes * \rightarrow * \stackrel{*_{Y}}{\rightarrow} Y$. Definimos $*_{[X, Y]_{*}}: * \rightarrow$ $[X, Y]_{*}$ como o adjunto da composição $* \otimes X \rightarrow * \stackrel{*_{Y}}{\longrightarrow} Y$.

Proposição 2.1.10. Seja $\mathcal{T}$ uma categoria monoidal (simétrica) fechada bicompleta. Então $\mathcal{T}_{*}$ equipada com o produto smash $\wedge$, a unidade $\mathbb{1} \sqcup *$ e o Hom interno pontuado $[-,-]_{*}$ é uma categoria monoidal (simétrica) fechada.

A demonstração é uma verificação direta dos axiomas e pode ser encontrada em [EM09, Teor. 4.20].

Também temos uma estrutura monoidal fechada induzida na categoria de morfismos de uma categoria monoidal fechada. 
Definição 2.1.11. Seja $\mathcal{T}$ uma categoria monoidal fechada bicompleta. O produto-pushout $\square$ em $\mathcal{T}^{\rightarrow}$ é o bifuntor

$$
\begin{aligned}
\square: \mathcal{T}^{\rightarrow} \times \mathcal{T}^{\rightarrow} & \rightarrow \mathcal{T}^{\rightarrow} \\
\left(f: X \rightarrow Y, f^{\prime}: X^{\prime} \rightarrow Y^{\prime}\right) & \mapsto\left(\left(f \otimes 1_{Y^{\prime}}, 1_{Y} \otimes f^{\prime}\right):\left(X \otimes Y^{\prime}\right) \coprod_{X \otimes X^{\prime}}\left(Y \otimes X^{\prime}\right) \rightarrow Y \otimes Y^{\prime}\right),
\end{aligned}
$$

e o Hom interno-pullback $[-,-]_{\square}$ em $\mathcal{T}^{\rightarrow}$ é o bifuntor

$$
\begin{aligned}
{[-,-]_{\square}: \mathcal{T}^{\rightarrow \text { op }} \times \mathcal{T}^{\rightarrow} } & \rightarrow \mathcal{T}^{\rightarrow} \\
\left(f^{\prime}: X^{\prime} \rightarrow Y^{\prime}, f: X \rightarrow Y\right) & \mapsto\left(\left(\left[f^{\prime}, 1_{X}\right],\left[1_{Y^{\prime}}, f\right]\right):\left[Y^{\prime}, X\right] \rightarrow\left[X^{\prime}, X\right] \times_{\left[X^{\prime}, Y\right]}\left[Y^{\prime}, Y\right]\right) .
\end{aligned}
$$

Proposição 2.1.12. Seja $\mathcal{T}$ uma categoria monoidal (simétrica) fechada bicompleta. Então $\mathcal{T}^{\rightarrow} e ́$ uma categoria monoidal ( simétrica) fechada.

Demonstração: Provaremos primeiro que $\square$ induz uma estrutura monoidal. Sejam $f: X \rightarrow$ $Y, f^{\prime}: X^{\prime} \rightarrow Y^{\prime}, f^{\prime \prime}: X^{\prime \prime} \rightarrow Y^{\prime \prime} \in \mathcal{T}^{\rightarrow}$. Temos pela proposição 2.1.8 que $\left(f \square f^{\prime}\right) \square f^{\prime \prime}$ é dado pelo morfismo do colimite do diagrama

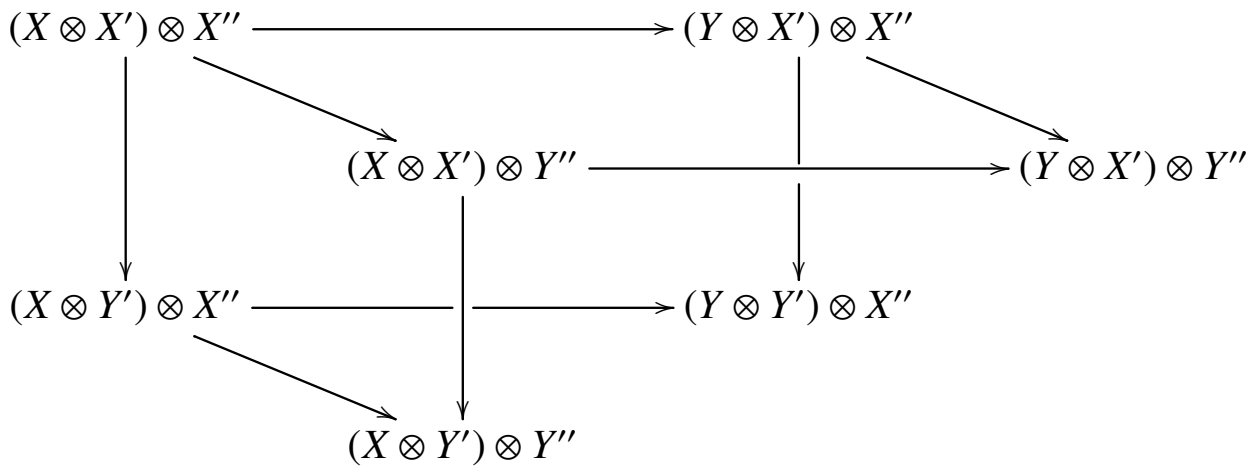

em $\left(Y \otimes Y^{\prime}\right) \otimes Y^{\prime \prime}$ dada pela propriedade universal. Temos então que $f \square\left(f^{\prime} \square f^{\prime \prime}\right)$ é dado de forma análoga e o associador em $\mathcal{T} \rightarrow$ é induzido pelo associador em $\mathcal{T}$. Podemos definir a unidade $\mathbb{1}^{\rightarrow}:=$ $\emptyset \rightarrow \mathbb{1}$ e é fácil verificar usando a proposição 2.1.8 que para qualquer $f: X \rightarrow Y \in \mathcal{T}^{\rightarrow}$ temos que $\mathbb{1}^{\rightarrow} \square f=1_{\mathbb{1}} \otimes f$ e $f \square \mathbb{1}^{\rightarrow}=f \otimes 1_{\mathbb{1}}$. Temos então que os unidadores à esquerda e à direita em $\mathcal{T}^{\rightarrow}$ são induzidos pelos unidadores em $\mathcal{T}$. Que as condições de coerência são satisfeitas segue delas serem satisfeitas em $\mathcal{T}$. A simetria de $\square$ também é induzida da simetria em $\mathcal{T}$.

Para $f^{\prime}: X^{\prime} \rightarrow Y^{\prime}$ a unidade da adjunção $\left(-\square f^{\prime} \dashv\left[f^{\prime},-\right]_{\square}\right)$ é dada por

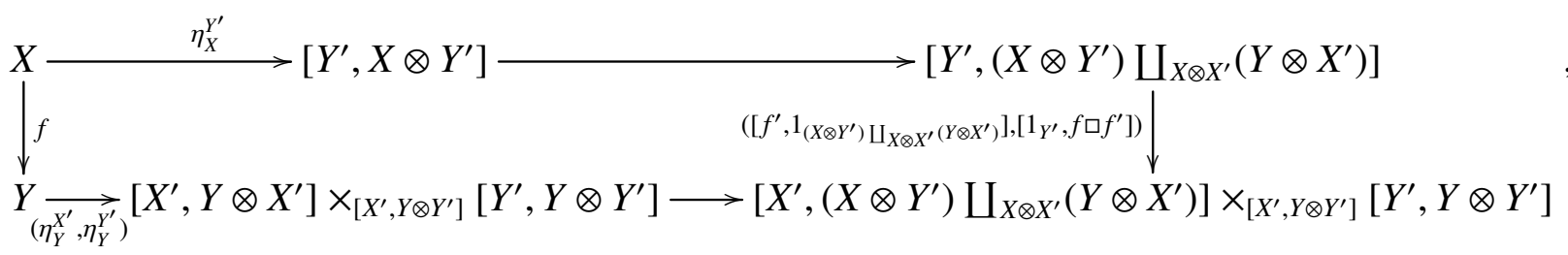


e a counidade é dada por

$$
\begin{aligned}
& \left(\left[X^{\prime}, X\right] \times_{\left[X^{\prime}, Y\right]}\left[Y^{\prime}, Y\right]\right) \otimes X^{\prime} \sqcup_{\left[Y^{\prime}, X\right] \otimes X^{\prime}}\left[Y^{\prime}, X\right] \otimes Y^{\prime} \longrightarrow\left[X^{\prime}, X\right] \otimes X^{\prime} \sqcup_{\left[Y^{\prime}, X\right] \otimes X^{\prime}}\left[Y^{\prime}, X\right] \otimes Y^{\prime} \stackrel{\left(\epsilon_{X}^{X^{\prime}}, \epsilon_{X}^{Y^{\prime}}\right)}{\longrightarrow} X . \\
& \downarrow\left(1_{\left[X^{\prime}, X\right] \times\left[X^{\prime}, Y\right]}\left[Y^{\prime}, Y\right] f^{\prime},\left[f^{\prime}, f\right]_{\square} \otimes 1_{Y^{\prime}}\right) \\
& \left(\left[X^{\prime}, X\right] \times_{\left[X^{\prime}, Y\right]}\left[Y^{\prime}, Y\right]\right) \otimes Y^{\prime} \\
& {\left[Y^{\prime}, Y\right] \otimes Y^{\prime}}
\end{aligned}
$$

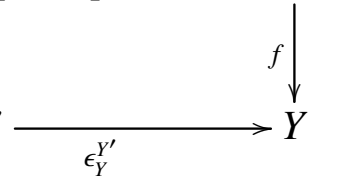

Que as equações de counidade-unidade são satisfeitas podem ser verificadas diretamente dessas definições.

\subsection{Categorias modelo monoidais}

Definimos agora o que significa uma estrutura monoidal fechada ser compatível com uma estrutura modelo e estudamos diversas propriedades úteis que seguem dessa compatibilidade.

Definição 2.2.1. Uma categoria modelo monoidal é uma categoria $\mathcal{T}$ equipada com ambas uma estrutura monoidal fechada e uma estrutura modelo tais que

1. axioma pushout-produto: Para $\iota, \iota^{\prime} \in C$ temos que $\iota \square \iota^{\prime} \in C$, e se ainda mais $\iota \in C \cap W$ ou $\iota^{\prime} \in C \cap W$ então $\iota \square \iota^{\prime} \in C \cap W$;

2. Axioma da unidade: $\mathbb{1} \in \mathcal{T}_{\text {Cof }}$.

Se a estrutura monoidal $\mathcal{T}$ for simetrica (cartesiana) então $\mathcal{T}$ é uma categoria modelo simetrica (cartesiana).

Proposição 2.2.2. Para todo objeto cofibrante $X$ de uma categoria modelo monoidal $-\otimes X$ preserva cofibrações e cofibrações triviais, e portanto a adjunção $(-\otimes X \dashv[X,-])$ é uma adjunção de Quillen.

Demonstração: Seja $\iota^{\prime} \in C\left(A^{\prime}, X^{\prime}\right)$. Pela proposição 2.1.8 temos que $\iota^{\prime} \square \emptyset_{X}=\iota^{\prime} \otimes X$, que pelo axioma pushout-produto é uma cofibração. Analogamente se $\iota^{\prime} \in C \cap W\left(A^{\prime}, X^{\prime}\right)$ então $\iota^{\prime} \otimes X \in C \cap W$. A segunda afirmação segue da definição de adjunção de Quillen.

Proposição 2.2.3. Seja $\mathcal{T}$ uma categoria modelo monoidal, $\iota \in C(A, X)$ e $p \in F(E, B)$. Então $[\iota, p]_{\square} \in F$. Se $\iota \in C \cap W$ então $[\iota, p]_{\square} \in F \cap W$. Em particular se $A \in \mathcal{T}_{\text {Cof }}$ e $Z \in \mathcal{T}_{\text {Fib }}$ então $[A, Z] \in \mathcal{T}_{\text {Fib }}$

Demonstração: Seja $\iota^{\prime} \in C \cap W\left(A^{\prime}, X^{\prime}\right)$. Então todo $(k, l) \in \mathcal{T}^{\rightarrow}\left(\iota^{\prime},[\iota, p]_{\square}\right)$ induz um morfismo $\left(\left(\epsilon_{E}^{X}(k \otimes X), \epsilon_{E}^{A}\left(\left(p_{[A, E]} l\right) \otimes A\right)\right), \epsilon_{B}^{X}\left(\left(p_{[X, B]} l\right) \otimes X\right)\right) \in \mathcal{T}^{\rightarrow}\left(\iota^{\prime} \square \iota, p\right)$. Como $p \in F$ temos pelo axioma pushout-produto um levantamento $H \in \mathcal{T}\left(X^{\prime} \otimes X, E\right)$, que por sua vez induz o levanta- 
mento $[X, H] \eta_{X^{\prime}}^{X} \in \mathcal{T}\left(X^{\prime},[X, E]\right)$ de $(k, l), \operatorname{logo}[\iota, p] \in F$.
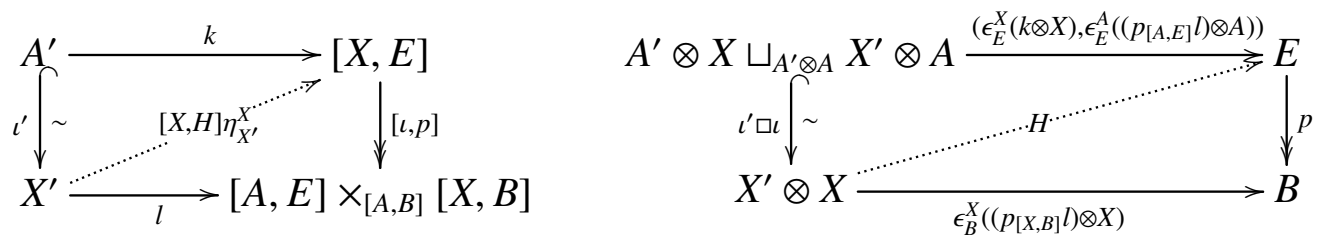

A segunda afirmação segue do argumento análogo com $\iota^{\prime} \in C$. A ultima afirmação segue do fato que para todo $Y \in \mathcal{T}$ temos $Y \otimes \emptyset=\emptyset$ pela proposição 2.1.8, e portanto pela adjunção $[\emptyset, Z]=*$.

Proposição 2.2.4. Seja $\mathcal{T}$ uma categoria modelo monoidal, $X, Y \in \mathcal{T}_{\text {Cof }}$ e $f \in \mathcal{T}(X, Y)$. Então $f \in W$ se e somente se para todo $Z \in \mathcal{T}_{\text {Fib }}$ temos $[f, Z] \in W$.

Demonstração: Suponha que $f \in C \cap W$. Então por 2.2.3 temos que $[f, Z] \in F \cap W \subset W$. Logo se $f \in W$ o lema de Ken Brown 1.3.6 nos dá $[f, Z] \in W$.

Suponha agora que para todo $Z \in \mathcal{T}_{\text {Fib }}$ temos $[f, Z] \in W$. Como pelo axioma da unidade temos que $\mathbb{1} \in \mathcal{T}_{\text {Cof }}$ e pela propriedade 2.2 .3 temos que $[Y, Z]$ e $[X, Z]$ são fibrantes então temos pelo lema 1.7.2.ii que $[f, Z]_{*}: \mathcal{H} o \mathcal{T}(\mathbb{1},[Y, Z]) \rightarrow \mathcal{H} o \mathcal{T}(\mathbb{1},[X, Z])$ é uma bijeção. Logo pela proposição 2.2.2 $f^{*}: \mathcal{H} o \mathcal{T}(Y, Z) \rightarrow \mathcal{H} o \mathcal{T}(X, Z)$ é uma bijeção, e portanto pelo lema 1.7.2.i temos que $f \in W$.

Proposição 2.2.5. Seja $\mathcal{T}$ uma categoria modelo monoidal (simétrica). Então $\mathcal{T}_{*}$ é uma categoria modelo monoidal ( simétrica).

Proposição 2.2.6. Seja $\mathcal{T}$ uma categoria monoidal (simétrica) fechada e uma categoria modelo cofibrantemente gerada por cofibrações I e cofibrações triviais J. Então se a unidade for cofibrante, $I \square I \subset C$ e $I \square J \subset C \cap W$ então $\mathcal{T}$ é uma categoria modelo monoidal (simétrica).

Demonstração: Temos por hipótese que $I \square I \subset{ }^{\square}(F \cap W)$. Pela estrutura monoidal fechada da proposição 2.1.12 isso implica que $I \subset{ }^{\square}[I, F \cap W]_{\square}$. Pelo lema 1.1.10 temos que $\operatorname{Cof}(I) \subset$ ${ }^{\square}[I, F \cap W]_{\square}$. Usando a adjunção e simetria também obtemos $I \subset{ }^{\square}[\operatorname{Cof}(I), F \cap W]_{\square}$ e novamente por 1.1.10 que $\operatorname{Cof}(I) \subset{ }^{\square}[\operatorname{Cof}(I), F \cap W]_{\square}$, e portanto pela adjunção $\operatorname{Cof}(I) \square \operatorname{Cof}(I) \subset{ }^{\square}(F \cap W)$. Usando um argumento análogo podemos provar que $\operatorname{Cof}(I) \square \operatorname{Cof}(J) \subset{ }^{\square} F$

Coends são um tipo de colimite que são úteis para definir diversas construções. Para uma revisão de aplicações de coend veja [Lo15]. Como veremos eles podem ser usados para definir o funtor de realização geométrica e monadas associadas à operads e 2-operads.

Definição 2.2.7. Sejam $\mathcal{T}$ uma categoria, $\mathcal{D}$ uma categoria pequena e $F: \mathcal{D}^{\mathrm{op}} \times \mathcal{D} \rightarrow \mathcal{T}$ um funtor. $\mathrm{O}$ coend de $F$ é o coequalizador:

$$
\int^{\mathcal{D}} F(D, D):=\text { Coeq }\left(\bigsqcup_{d \in \underset{D, D^{\prime} \in \mathcal{D}}{\amalg}} \coprod_{\mathcal{D}\left(D, D^{\prime}\right)} F\left(D^{\prime}, D\right) \rightrightarrows \coprod_{D \in \mathcal{D}} F(D, D)\right)
$$


A seguinte propriedade de coends de diagramas de Reedy generalizado dualizáveis em categorias modelo monoidais cofibrantemente geradas vai ser útil.

Proposição 2.2.8. Seja $\mathcal{T}$ uma categoria modelo monoidal cofibrantemente gerada, $\mathcal{R}$ uma categoria de Reedy generalizada dualizável, $\iota^{-} \in C^{\mathcal{R}^{o p}}\left(A^{-}, X^{-}\right) e \iota^{\prime-} \in C^{\mathcal{R}}\left(A^{\prime-}, X^{--}\right)$. Então

$$
\int^{\mathcal{R}} \iota^{R} \square \iota^{\prime R} \in C_{\mathcal{T}}\left(\int^{\mathcal{R}} A^{R} \otimes X^{\prime R} \sqcup_{\int^{\mathcal{R}} A^{R} \otimes A^{\prime R}} \int^{\mathcal{R}} X^{R} \otimes A^{\prime R}, \int^{\mathcal{R}} X^{R} \otimes X^{\prime R}\right) .
$$

Além disso se $\iota \in C^{\mathcal{R}^{o p}} \cap W^{\mathcal{R}^{o p}}$ ou $\iota^{\prime} \in C^{\mathcal{R}} \cap W^{\mathcal{R}}$ então $\int^{\mathcal{R}} \iota^{R} \square \iota^{\prime R} \in C_{\mathcal{T}} \cap W_{\mathcal{T}}$.

Demonstração: Sejam $p \in F_{\mathcal{T}}(E, B)$ e $(u, v) \in \mathcal{T}^{\rightarrow}\left(\int^{\mathcal{R}} \iota^{R} \square \iota^{\prime R}, p\right)$. Construímos indutivamente um levantamento de $(u, v)$. Primeiro note que como colimites comutam entre si temos que

$$
\int^{\mathcal{R}} A^{R} \otimes X^{\prime R} \sqcup_{\int^{\mathcal{R}} A^{R} \otimes A^{\prime R}} \int^{\mathcal{R}} X^{R} \otimes A^{\prime R}=\int^{\mathcal{R}} A^{R} \otimes X^{\prime R} \sqcup_{A^{R} \otimes A^{\prime R}} X^{R} \otimes A^{\prime R} .
$$

Seja $R \in \mathcal{R}$ tal que $\operatorname{gr}(R)=0$. Temos então um diagrama comutativo

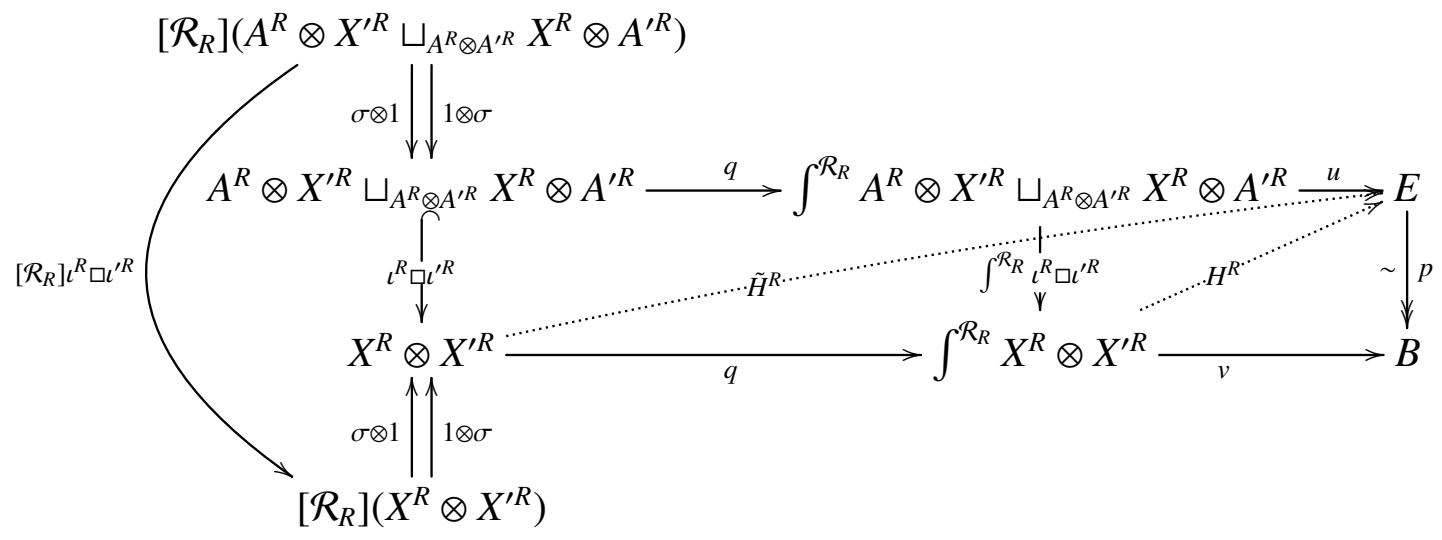

Como $\iota^{\prime R} \in C_{\mathcal{T}^{\mathcal{R}_{R}}},\left[\iota^{R}, p\right]_{\square} \in \mathcal{T}^{\mathcal{R}_{R}}\left(\left[X^{R}, E\right],\left[A^{R}, E\right] \times_{\left[A^{R}, B\right]}\left[X^{R}, B\right]\right)$ e pela proposição 2.2 .3 temos que $\left[\iota^{R}, p\right]_{\square} \in F_{\mathcal{T}^{R_{R}}}$ então existe um levantamento $\tilde{H}^{R} \in \mathcal{T}\left(X^{R} \otimes X^{\prime R}, E\right)$ de $(u q, v q)$ que coequaliza os morfismos $1 \otimes \sigma$ e $\sigma \otimes 1$, e portanto $\tilde{H}^{R}$ induz um levantamento $H^{R} \in \mathcal{T}\left(\int^{\mathcal{R}_{R}} X^{R} \otimes X^{\prime R}, E\right)$ de $(u, v)$. Temos portanto uma aplicação $H^{0} \in \mathcal{T}\left(\int^{F^{0} \mathcal{R}} X^{R} \otimes X^{\prime R}, E\right)$ que é um levantamento da restrição de $(u, v)$ na 0 -filtração.

Suponha agora que construímos $H^{q-1} \in \mathcal{T}\left(\int^{F^{q-1} \mathcal{R}} X^{R} \otimes X^{\prime R}, E\right)$ que é um levantamento da restrição de $(u, v)$ na $q-1$-filtração. Para todo $R \in \mathcal{R} \operatorname{com} \operatorname{gr}(R)=q$ temos pelo mesmo argumento acima um levantamento $H^{R}\left(\int^{\mathcal{R}_{R}} X^{R} \otimes X^{\prime R}, E\right)$ da restrição $(u, v) \in \mathcal{T}^{\rightarrow}\left(\int^{\mathcal{R}_{R}}\left(\iota^{R} \square l_{X}^{R}\right) \square\left(\iota^{\prime R} \square l_{X^{\prime}}^{R}\right), p\right)$, aonde

$$
\begin{gathered}
\int^{\mathcal{R}_{R}}\left(\iota^{R} \square l_{X}^{R}\right) \square\left(\iota^{\prime R} \square l_{X^{\prime}}^{R}\right) \in \\
\mathcal{T}\left(\int^{\mathcal{R}_{R}}\left(A_{L A^{R}} L X^{R}\right) \otimes X^{\prime R} \sqcup_{\left(A^{R} \sqcup_{L A^{R}} L X^{R}\right) \otimes\left(A^{\prime R} \sqcup_{L A^{\prime R}} L X^{\prime R}\right)} X^{R} \otimes\left(A^{\prime R} \sqcup_{L A^{\prime R}} L X^{\prime R}\right), \int^{\mathcal{R}_{R}} X^{R} \otimes X^{\prime R}\right)
\end{gathered}
$$




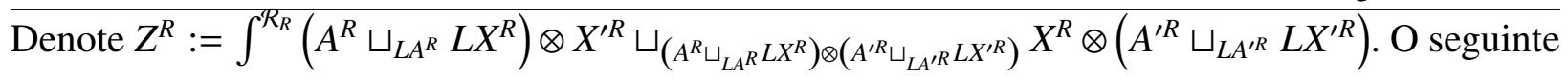
quadrado é um pushout:

$$
\begin{aligned}
& \left(\sqcup_{R \in F^{q} \mathcal{R} \backslash F^{q-1} \mathcal{R}^{R}} Z^{R}\right) \sqcup \int^{F^{q-1} \mathcal{R}} A^{R} \otimes X^{\prime R} \sqcup_{A^{R} \otimes A^{\prime R}} X^{R} \otimes A^{\prime R} \longrightarrow \int^{F^{q} \mathcal{R}} A^{R} \otimes X^{\prime R} \sqcup_{A^{R} \otimes A^{\prime R}} X^{R} \otimes A^{\prime R} .
\end{aligned}
$$

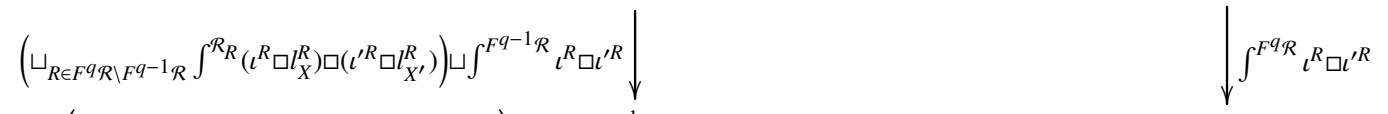

$$
\begin{aligned}
& \left(\sqcup_{R \in F^{q} \mathcal{R} \backslash F^{q-1} \mathcal{R}} \int^{\mathcal{R}_{R}} X^{R} \otimes X^{\prime R}\right) \sqcup \int^{F^{q-1} \mathcal{R}} X^{R} \otimes X^{\prime R} \longrightarrow \int^{F^{q} \mathcal{R}} X^{R} \otimes X^{\prime R}
\end{aligned}
$$

Logo a hipótese de indução e as construções nas demonstrações de 1.1.10.iv) e 1.1.10.v) nos dão $H^{q} \in \mathcal{T}\left(\int^{F^{q} \mathcal{R}} X^{R} \otimes X^{\prime R}, E\right)$ que é um levantamento da restrição de $(u, v)$ na $q$-filtração.

A segunda afirmação segue do argumento análogo assumindo que $p \in F_{\mathcal{T}} \cap W_{\mathcal{T}}$.

A construção da estrutura modelo de operads e 2-operads usam a noção de um objeto intervalo de Hopf [BM07] para definir funtorialmente objetos de caminhos.

Definição 2.2.9. Em uma categoria modelo monoidal $\mathcal{T}$ um objeto intervalo é um objeto $I \in \mathcal{T}$ equipado com uma fatoração em uma cofibração seguida de uma equivalência fraca

$$
\mathbb{1} \sqcup \mathbb{1} \stackrel{(0,1)}{\longrightarrow} I \stackrel{\varepsilon}{\sim} \mathbb{1}
$$

do morfismo codiagonal da unidade $\mathbb{1}$, equipada com uma estrutura de monoide comutativo $\left(I, \mu_{I}, 0\right)$ tal que 0 é a unidade, 1 é um elemento absorvente e $\varepsilon$ é uma counidade do monoide, i.e. tal que os seguintes diagramas comutam:
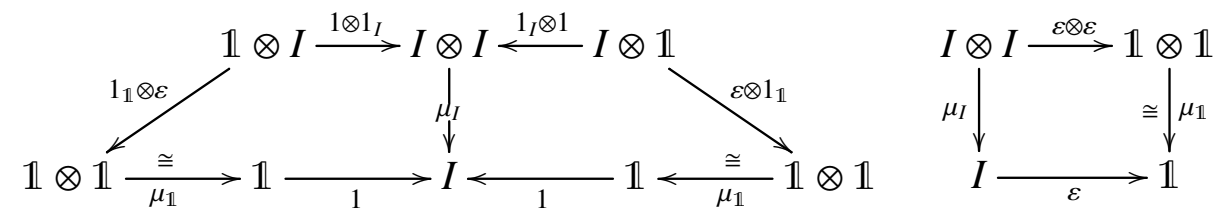

Um objeto intervalo (cocommutativo) de Hopf é um objeto intervalo I equipado com uma estrutura de comonoide (cocommutativo) tal que os morfismos na fatoração da codiagonal da unidade são morfismos de comonoides.

Em Top o intervalo $I=[0,1]$ com o máximo como a estrutura de monoide e a estrutura comonoidal diagonal é um objeto intervalo de Hopf cocomutativo.

\subsection{Monadas e álgebras}

Uma monada pode ser definida de uma forma compacta como sendo um monoide na categoria de endofuntores de uma categoria.

Definição 2.3.1. Seja $\mathcal{T}$ uma categoria. A categoria monoidal de endofuntores de $\mathcal{T}$ é a categoria $\operatorname{End}(\mathcal{T})$, cujos objetos são funtores $F: \mathcal{T} \rightarrow \mathcal{T}$ e cujos morfismos são transformações naturais, 
equipada com composições como o produto tensorial e o funtor identidade como identidade.

Definição 2.3.2. Seja $\mathcal{T}$ uma categoria. Uma monada em $\mathcal{T}$ é um monoide $(C, \mu, \eta)$ em $\operatorname{End}(\mathcal{T})$.

Podemos usar as definições na sessão 2.1 para desempacotar a definição de uma monada como um endofuntor equipado com um par de transformações naturais satisfazendo certas condições de coerência. Uma monada define uma teoria algébrica na categoria subjacente, com as imagens do endofuntor subjacente da monada sendo objetos livres da teoria algébrica. Uma monada define uma categoria de álgebras dada pela seguinte definição.

Definição 2.3.3. Seja $\mathcal{T}$ uma categoria e $C$ uma monada em $\mathcal{T}$. Uma $C$-algebra é um objeto $X \in \mathcal{T}$ equipado com um morfismo $\xi \in \mathcal{T}(C X, X)$ tais que os diagramas abaixo comutam:
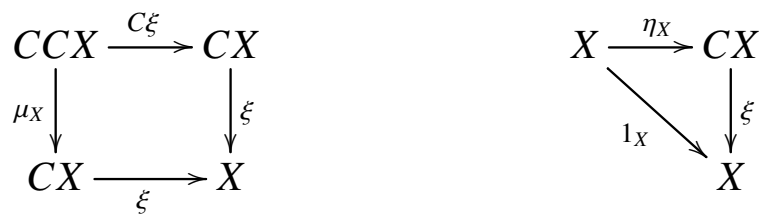

Um $C$-morfismo entre $C$-álgebras é um morfismo entre os objetos subjacentes que comuta com os morfismos estruturais.

Denotamos a categoria de $C$-álgebras e $C$-morfismos por $C[\mathcal{T}]$.

Note que para qualquer $X \in \mathcal{T}$ a multiplicação $\mu$ de $C$ induz uma estrutura de $C$-álgebra em $C X$, i.e. $C X \in C[\mathcal{T}]$. Logo $C$ define um funtor $C: \mathcal{T} \rightarrow C[\mathcal{T}]$. Esse funtor é adjunto à esquerda do funtor esquecimento, com a unidade induzida pela unidade $\eta$ de $C$ e a counidade dada pelos morfismos estruturais de $C$-álgebras.

Também definimos $C$-funtores, que podem ser pensados ou como funtores que preservam a estrutura algébrica de $C$ ou como um $C$-módulo à direita.

Definição 2.3.4. Seja $\mathcal{T}$ uma categoria e $C$ uma monada em $\mathcal{T}$. Um $C$-funtor em $\mathcal{A}$ é um funtor $F: \mathcal{T} \Rightarrow \mathcal{A}$ equipado com uma transformação natural $\lambda: F \circ C \rightarrow F$ tais que os diagramas abaixo comutam:
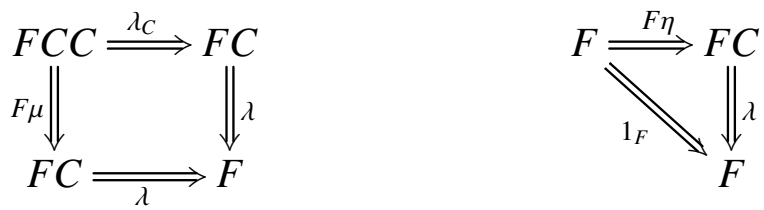

Um morfismo de $C$-functores em $\mathcal{A}$ é uma transformação natural entre os funtores subjacentes que comuta com as transformações naturais estruturais.

Precisaremos dos seguintes exemplos:

1. Se $(C, \mu, v)$ é uma monada então $(C, \mu)$ é um $C$-funtor. 
2. Se $\phi:(C, \mu, v) \Rightarrow\left(C^{\prime}, \mu^{\prime}, v^{\prime}\right)$ é um morfismo de monadas e $(F, \lambda)$ é um $C^{\prime}$-funtor então $(F, \lambda F \phi)$ é um $C$-funtor. Em particular, pelo exemplo anterior, $\left(C^{\prime}, \mu^{\prime} C^{\prime} \phi\right)$ é um $C$-funtor. Também temos que $\phi:(C, \mu) \Rightarrow\left(C^{\prime}, \mu^{\prime} C^{\prime}(\phi)\right)$ é um morfismo de $C$-funtores.

3. Seja $(S \dashv \Lambda): \mathcal{T} \rightleftharpoons \mathcal{A}$ uma adjunção com unidade $\eta: I d_{\mathcal{T}} \Rightarrow \Lambda S$ e counidade $\epsilon: S \Lambda \Rightarrow$ $I d_{\mathcal{A}}$. Então $\left(\Lambda S, \Lambda \epsilon_{S}, \eta\right)$ é uma monada em $\mathcal{T}$ e $\left(S, \epsilon_{S}\right)$ é um $\Lambda S$-funtor.

4. Se $\alpha:(C, \mu, v) \Rightarrow\left(\Lambda S, \Lambda \epsilon_{S}, \eta\right)$ é um morfismo de monadas, com $\Lambda S$ como no exemplo anterior, então $S$ é um $C$-funtor em $\mathcal{A}$ e $\alpha:(C, \mu) \Rightarrow\left(\Lambda S, \Lambda \epsilon_{S} S \alpha\right)$ é um morfismo de $C$-funtores em $\mathcal{T}$.

A construção bar bilateral descrita aqui foi introduzida em [Ma72] e admite diversas aplicações. A construção dos espaços e aplicações na demonstração do teorema do reconhecimento dependem dessa construção.

Definição 2.3.5. A construção bar bilateral é a categoria $B(\mathcal{T}, \mathcal{A})$ e o funtor $B^{\bullet}: B(\mathcal{T}, \mathcal{A}) \rightarrow \mathcal{A}^{\Delta^{o p}}$ definidos da seguinte forma: Os objetos de $B(\mathcal{T}, \mathcal{A})$ são triplas $((F, \lambda),(C, \mu, v),(X, \xi))$, abreviadas como $(F, C, X)$, aonde $C$ é uma monada em $\mathcal{T}, F$ é um $C$-funtor em $\mathcal{A}$ e $X$ é uma $C$-álgebra, e morfismos $(\alpha, \phi, f) \in B(\mathcal{T}, \mathcal{A})\left((F, C, X),\left(F^{\prime}, C^{\prime}, X^{\prime}\right)\right)$ são triplas com $\phi$ um morfismo de monadas, $f$ um $C$-morfismo e $\alpha$ é um morfismo de $C$-funtores.

O funtor $B^{\bullet}(F, C, X)$ é definido por

$$
\begin{aligned}
& B .: B(\mathcal{T}, \mathcal{A}) \rightarrow \mathcal{A}^{\Delta^{\text {op }}} \\
&(F, C, X) \mapsto\left(\begin{array}{rl}
F C^{\bullet} X, & \partial_{i}=\left\{\begin{array}{ll}
\lambda_{C^{\bullet-1}}, & i=0 \\
F C^{i-1} \mu_{C^{\bullet-i}}, & 0<i<\bullet \\
F C^{\bullet-1} \rho, & i=\bullet \\
s_{i} & =F C^{i} \eta_{C^{\bullet-i+1}}, 0 \leq i \leq \bullet
\end{array}\right)
\end{array}\right.
\end{aligned}
$$

Propriedade básicas dessa construção podem ser encontradas em [Ma72, C.9]. 



\section{Capítulo 3}

\section{Espaços topológicos, espaços de laços e espectros}

Neste capítulo apresentamos a categoria de espaços topológicos em que vamos trabalhar. A categoria de todos espaços topológicos admite uma estrutura cartesiana e uma estrutura modelo, porém a estrutura cartesiana não é fechada e assim essa categoria não é uma categoria modelo monoidal. Por esse motivo trabalhamos na subcategoria de espaços compactamente gerados fracamente Hausdorff que é bicompleta e admite uma estrutura cartesiana fechada compatível com a estrutura modelo que a torna uma categoria modelo cartesiana.

Na primeira sessão apresentamos a categoria de espaços compactamente gerados fracamente Hausdorff e apresentamos a sua estrutura de categoria modelo cartesiana [StN09, Hi15].

Na segunda sessão apresentamos a construção dos funtores de $N$-espaços de laços $\Omega^{N}$ e de seus adjuntos de Quillen à esquerda, os funtores de espaços de $N$-suspensões $\Sigma^{N}$. Para definir o funtor de espaços de laços infinitos $\Omega^{\infty}$ usamos a categoria de espectros. Apresentamos a estrutura modelo estável na categoria de espectros [BF78, Sc97] tal que o funtor $\Omega^{\infty}$ admite um adjunto de Quillen à esquerda $\Sigma^{\infty}$.

Na terceira sessão damos a definição de espaços relativos, sua estrutura modelo de Reedy natural, dos funtores de espaços de $N$-laços relativos $\Omega_{\mathrm{Rel}}^{N}$ de um espaço relativo, i.e. uma aplicação de espaços pontuados, e dos funtores de 2-espaços de $N$-laços relativos $\Omega_{2}^{N}$ de um espaço relativo, que é simplesmente o par formado pelo espaço de $N$-laços do codomínio e o espaço de $N$-laços relativos do espaço relativo. Provamos então que $\Omega_{2}^{N}$ admite um adjunto de Quillen fraco à esquerda. Também damos uma estrutura modelo cuja categoria homotópica é a subcategoria correflexiva de espaços relativos $N$ - 1-conexos. Para definir o 2-funtor de espaço de laços infinitos $\Omega_{2}^{\infty}$ introduzimos a categoria de espectros relativos composta de pares de espectros equipados com uma sequência de aplicações entre o par que aumenta o índice em 1. Mostramos que essa categoria também admite uma estrutura modelo estável e que $\Omega_{2}^{\infty}$ admite um adjunto de Quillen fraco à esquerda.

Na quarta sessão apresentamos a construção de realizações geométricas de espaços simpliciais. 


\subsection{Espaços compactamente gerados fracamente Hausdorff}

Vai ser útil pensarmos numa topologia de um espaço $X$ como sendo especificada por subcon-

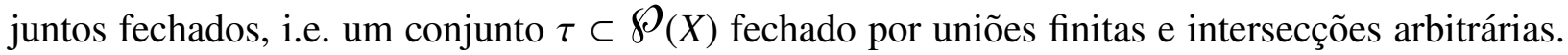
Para detalhes sobre espaços compactamente gerados fracamente Hausdorff veja [StN09]. Denotamos a categoria de todos os espaços topológicos por Top.

Definição 3.1.1. Um subconjunto $U$ de um espaço topológico $X$ com topologia $\tau$ é $k$-fechado se para todo espaço Hausdorff compacto $K$ e aplicação $t \in \operatorname{Top}(K, X)$ o subconjunto $t^{-1}(U) \subset K$ é fechado. Escrevemo $k(\tau)$ para a coleção de subconjuntos $k$-fechados. Um espaço $X$ é compactamente gerado se $k(\tau)=\tau$.

Denotamos por $k(X)$ o espaço topológico $X$ com a topologia $k(\tau)$. Note que para um espaço topológico arbitrário $X$ sempre temos $\tau \subset k(\tau)$, ou seja os subconjuntos $k$-fechados de $X$ nos dão um refinamento da topologia de $X$.

Definição 3.1.2. Um espaço $X$ é fracamente Hausdorff se para todo espaço Hausdorff compacto $K$ e aplicação $t \in \mathbf{T o p}(K, X)$ a imagem $t(K) \subset X$ é fechada.

Um espaço compactamente gerado $X$ é fracamente Hausdorff se e somente se a imagem de $\nabla_{X} \in \operatorname{Top}(X, X \times X)$ for fechada. Seja $E$ uma relação de equivalência em um espaço topológico compactamente gerado $X$. Então $X_{/ E}$ é compactamente gerado e fracamente Hausdorff se e somente se $E$ for fechada, i.e. se $E$ for fechada em $X \times X$. Para todo espaço compactamente gerado $X$ existe a menor relação de equivalência fechada $E_{\min }$ em $X$, e $X_{/ E_{\min }}$ é um espaço compactamente gerado e fracamente Hausdorff, que denotamos por $h(X)$.

Seja Top $\subset$ Top a subcategoria plena dos espaços topológicos compactamente gerados e fracamente Hausdorff. O produto de espaços compactamente gerados não é necessariamente compactamente gerado. Por esse motivo, denotando o produto usual de espaços topológicos por $\times_{0}$, definimos o produto compactamente gerado de espaços topológicos $X$ e $Y$ por $X \times Y:=k\left(X \times_{0} Y\right)$. Esse produto compactamente gerado define uma estrutura cartesiana em Top. Sejam $X$ e $Y$ espaços topológicos e $[X, Y]_{0}$ o espaço de aplicações contínuas entre $X$ e $Y$ com a topologia compacta-aberta. Definimos $[X, Y]:=k\left([X, Y]_{0}\right)$. Se $X$ é um espaço compactamente gerado e fracamente Hausdorff e $Y$ um espaço compactamente gerado então $[X, Y]$ é compactamente gerado e fracamente Hausdorff. Além disso temos para todo espaço compactamente gerado e fracamente Hausdorff uma adjunção $(-\times X \dashv[X,-])$ dada pela unidade $\eta^{X}: I d_{\text {Top }} \Rightarrow[X,-\times X] \operatorname{com} \eta_{Y}^{X}(y)=(x \mapsto(y, x)) \mathrm{e}$ pela counidade $\epsilon^{X}:[X,-] \times X \Rightarrow I d_{\text {Top }} \operatorname{com} \epsilon_{Y}^{X}(f, x)=f(x)$. Logo Top é uma categoria cartesiana fechada.

Note que Top contém todos os CW-complexos, em particular ele contém as esferas. A categoria cartesiana Top também tem a propriedade que produtos de $\mathrm{CW}$-complexos arbitrários são CW-complexos, o que não é verdade em Top. Como Top possui objeto final $*$ temos a categoria 
pontuada $\mathrm{Top}_{*}$. Podemos então definir para $X \in \mathrm{Top}$ o conjunto $\pi_{0} X$ de componentes conexas por caminhos de $X$ e para cada $q \in \mathbb{N}$ com $q>0$ podemos definir os grupos de homotopia $\pi_{q}(X, x):=\pi_{0}\left(\left[\mathbb{S}^{q}, X\right]_{*}\right)$. A estrutura de grupo é induzida da aplicação em $\operatorname{Top}\left(\mathbb{S}^{q}, \mathbb{S}^{q} \vee \mathbb{S}^{q}\right)$ obtida colapsando o equador da esfera, que pelo argumento de Eckmann-Hilton é abeliano para $q>1$.

Definição 3.1.3. Uma equivalência homotópica fraca é uma aplicação $f \in \operatorname{Top}(X, Y)$ tal que a função induzida $f_{*} \in \operatorname{Set}\left(\pi_{0} X, \pi_{0} Y\right)$ e os homomorfismos induzidos $f_{*} \in \operatorname{Grp}\left(\pi_{q} X, \pi_{q} Y\right)$ são isomorfismos para todo $q \in \mathbb{N}$.

A estrutura modelo de Quillen [Hi15] é uma estrutura modelo em Top cofibrantemente gerada aonde as equivalências fracas são as equivalências homotópicas fracas. Seja $I=[0,1]$ e identifique $\mathbb{S}^{q}$ com o bordo de $I^{q+1}$.

Teorema 3.1.4. A categoria Top com as classes de equivalências homotópicas fracas $W_{Q}$ como equivalências fracas, fibrações de Serre $F_{Q}$ como fibrações e retrações de $C W$-complexos relativos $C_{Q}$ como cofibrações é uma categoria modelo cartesiana própria cofibrantemente gerada pelos conjuntos de cofibrações geradoras $I_{Q}:=\left(i^{q}\right)_{q \in \mathbb{N}} \in \prod_{q \in \mathbb{N}}$ Top $\left(\mathbb{S}^{q-1}, I^{q}\right)$ dada pelas inclusões dos bordos e cofibrações triviais geradoras $J_{Q}:=\left(j^{q}\right)_{q \in \mathbb{N}} \in \prod_{q \in \mathbb{N}} \operatorname{Top}\left(I^{q}, I^{q+1}\right)$ dadas por $j^{q}(t):=$ $(0, t)$.

Demonstração: Os colimites na categoria de todos os espaços topológicos de um diagrama de espaços compactamente gerados e fracamente Hausdorff pode não ser fracamente Hausdorff. O colimite de um diagrama $X \in \mathrm{T}^{\circ} \mathrm{p}^{C}$ é dado por $h\left(\operatorname{Colim}_{c \in C}^{\text {Esp Top }} X(c)\right)$, i.e. o colimite como espaços topológicos quocientado pela menor relação de equivalência fechada. A categoria Top também é completa e os limites são preservados pelo funtor de esquecimento na categoria de conjuntos. Dado um diagrama $X \in \mathrm{Top}^{C}$ o limite, como conjunto, é um subconjunto $\operatorname{Lim}_{c \in C}^{\mathrm{Conj}} X(c) \subset$ $\prod_{c \in C} X(c)$. Podemos equipar $\prod_{c \in C} X(c)$ com a topologia produto compactamente gerada e equipamos $\operatorname{Lim}_{c \in C}^{\mathrm{Conj}} X(c)$ com a topologia de subespaço. Logo Top é bicompleta. Para detalhes veja [StN09].

Por definição $J_{Q}^{\square}$ são as fibrações de Serre e $\operatorname{Cel}\left(I_{Q}\right)$ são os $\mathrm{CW}$-complexos relativos, e portanto $\operatorname{Cof}\left(I_{Q}\right)$ são as retrações de $\mathrm{CW}$-complexos relativos. As classes de morfismos do enunciado satisfazem as condições da proposição 1.4.6 [Hi15].

Note que $\operatorname{Cel}\left(J_{Q}\right) \subset \operatorname{Cel}\left(I_{Q}\right)$ já que $J_{Q} \subset \operatorname{Cel}\left(I_{Q}\right)$. Como todo subespaço compacto de um CWcomplexo relativo intersecta um numero finito de células os domínios de $I_{Q}\left(J_{Q}\right)$ são $\mathbb{N}$-pequenos em relação à $\operatorname{Cel}\left(I_{Q}\right)\left(\operatorname{Cel}\left(J_{Q}\right)\right)$.

Como todo pushout de morfismos em $J_{Q}$ é uma equivalência homotópica, e portanto uma equivalência homotópica fraca, e a composição transfinita de isomorfismos de grupos é um isomorfismo de grupo então $\operatorname{Cel}\left(J_{Q}\right) \subset W_{Q}$. Além disso retrações de equivalências homotópicas fracas são equivalências homotópicas fracas já que retrações de isomorfismos são isomorfismos (em particular na categoria de grupos). Logo pelo argumento do objeto pequeno ${ }^{\square}\left(J_{Q}^{\square}\right)=\operatorname{Cof}\left(J_{Q}\right) \subset$ $\operatorname{Cof}\left(I_{Q}\right) \cap W_{Q}={ }^{\square}\left(I_{Q}^{\square}\right) \cap W_{Q}$. 
Seja $p \in I_{Q}^{\square}(E, B)$. Como os elementos de $\pi_{q}(E)$ são representados por aplicações $\mathbb{S}^{q} \rightarrow E$ a existência dos levantamentos dos $i^{q}$ implicam que $\pi_{q}(p)$ é injetora para todo $q \in \mathbb{N}$. Além disso os elementos de $\pi_{q}(B)$ podem ser representado por um diagrama da forma

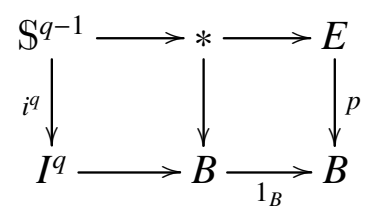

cujos levantamentos nos dão a sobrejeção dos $\pi_{q}(p)$. Logo $p \in W$. Pelo argumento do objeto pequeno $I_{Q}^{\square}=\operatorname{Cof}\left(I_{Q}\right)^{\square} \subset \operatorname{Cof}\left(J_{Q}\right)^{\square}=J_{Q}^{\square}$, e portanto $I_{Q}^{\square} \subset W \cap J_{Q}^{\square}$.

Suponha agora que $p \in J_{Q}^{\square} \cap W_{Q}(E, B)$, ou seja que $p$ é uma fibração de Serre trivial. Seja $(k, l) \in \operatorname{Top}^{\rightarrow}\left(i^{q}, p\right):$

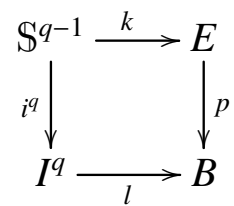

O levantamento existe nos casos $q=0$ por $\pi_{0}(p)$ ser sobrejetora e $p$ uma fibração de Serre e $q=1$ por $\pi_{0}(p)$ ser injetora. Suponhamos que $q>1$. Por $p \in W_{Q}$ temos $\pi_{q-1}(p)$ injetora e portanto existe $H \in \mathrm{Top}\left(I^{q+1}, E\right)$ tal que $H i_{q}=k$, porém não necessariamente $p H=l$. Considere o diagrama abaixo, aonde o quadrado da esquerda é um pushout.

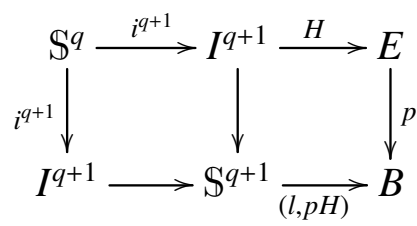

De novo por $p \in W_{Q}$ temos $(\tilde{H}, H) \in \mathrm{Top}\left(\mathbb{S}^{q+1}, E\right)$ um levantamento de $(H,(l, p H))$. Portanto temos $[(p \tilde{H}, l)]=[(p \tilde{H}, p H)]+[(p H, l)]=[(l, p H)]+[(p H, l)]=0$ em $\pi_{q+1}(B)$. Portanto temos uma homotopia $\phi \in \operatorname{Top}\left(I^{q+1}, B\right)$ de $l$ em $p \tilde{H}$ que fixa o bordo de $I^{q}$. Por $p$ ser uma fibração de Serre temos um levantamento $\bar{H} \in \operatorname{Top}\left(I^{q+1}, E\right)$ de $(\tilde{H}, \phi)$

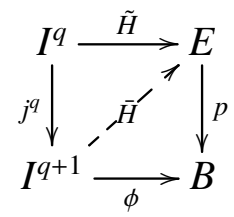

e $\bar{H} \uparrow_{\{1\} \times I^{q}} \in \operatorname{Top}\left(I^{q}, E\right)$ é um levantamento de $(k, l)$. Logo $W_{Q} \cap J_{Q}^{\square} \subset I_{Q}^{\square}$.

A unidade do produto cartesiano é o espaço terminal $*$ que é um CW-complexo, e portanto cofibrante. Pela proposição 2.2.6 para provarmos que Top é uma categoria modelo cartesiana basta provarmos que o axioma produto-pushout vale para elementos das cofibrações e cofibrações triviais geradoras. Pela definição podemos ver que para $i^{p}, i^{q} \in I_{Q}$ temos que $i^{p} \square i^{q}$ é homeomorfo a 
$\overline{i^{p+q}}$, e se $j^{q} \in J_{Q}$ então $i^{p} \square j^{q}$ é homeomorfo a $j^{p+q}$. Logo Top é uma categoria modelo cartesiana.

É fácil verificar que todos os objetos de Top são fibrantes, logo pelo corolário 1.7.4 a estrutura modelo de Top é própria à direita. Que Top é próprio à esquerda segue do fato que equivalências fracas são preservadas por pushouts ao longo de uma inclusão de um espaço $X$ em um espaço obtido colando uma $q$-célula em $X$, e portanto são preservadas por pushouts ao longo de morfismos em $\operatorname{Cel}\left(I_{Q}\right)$, e que se equivalências fracas são preservadas por pushouts ao longo de uma classe de morfismos então elas também são preservadas por pushouts ao longo de retratos dos morfismos nessa classe. Para detalhes veja [Hi09, Teor. 13.1.10]..

Corolário 3.1.5. A categoria pontuada Top ${ }_{*}$ é uma categoria modelo simétrica cofibrantemente gerada própria.

Demonstração: Que Top ${ }_{*}$ é uma categoria modelo cofibrantemente gerada segue da proposição 1.5.3, e que ela é própria segue da proposição 1.7.6. Que ela é uma categoria modelo simétrica segue da proposição 2.2 .5

Existe uma outra estrutura modelo em Top em que as equivalências fracas são as equivalências homotópicas e as fibrações são as fibrações de Hurewicz, a estrutura modelo de Strøm [StA72]. Essa estrutura modelo não é cofibrantemente gerada, portanto a maioria dos resultados aqui não valem para essa estrutura modelo. Porém as cofibrações dessa estrutura modelo aparecem em algumas hipóteses nessa tese e portanto damos a definição delas aqui.

Definição 3.1.6. Para $A, X \in$ Top tal que $A$ é um subespaço fechado de $X$ dizemos que o par $(A, X)$ é um par de retrato por deformação de vizinhança, ou par-RDV, se existem aplicações $u \in \operatorname{Top}(X, I)$ e $H \in \operatorname{Top}(X \times I, X)$ tais que $u^{-1}(0) \subset A, H(x, 0)=x$ para todo $x \in X, H(a, t)=a$ para todo $t \in I$ e $a \in A$, e $H(x, 1) \in A$ para todo $x \in u^{-1}([0,1))$.

Note que as cofibrações de Quillen são cofibrações de Strøm. Também precisaremos da definição dos objetos cofibrantes nessa estrutura modelo na categoria de espaços pontuados.

Definição 3.1.7. Um espaço pontuado $X \in \mathrm{T} \circ \mathrm{p}_{*}$ é bem pontuado se $\left(X_{*}, X\right)$ é um par-RDV.

\subsection{Espaços de laços e espectros}

Seja $N \in \mathbb{N} \operatorname{com} N>0$ e identifique a $N$-esfera $\mathbb{S}^{N} \operatorname{com}$ o espaço pontuado $I^{N} / \partial I^{N}$ com ponto base a classe de equivalência dos pontos do bordo $\partial I^{N}$.

Definição 3.2.1. O funtor de espaços de N-laços é

$$
\begin{aligned}
\Omega^{N}: \mathrm{Top}_{*} & \rightarrow \mathrm{Top}_{*} \\
X & \mapsto\left[\mathbb{S}^{N}, X\right]_{*}
\end{aligned}
$$

Esses funtores admitem adjuntos de Quillen à esquerda. 
Definição 3.2.2. O funtor de espaços de $N$-suspensões é

$$
\begin{aligned}
\Sigma^{N}: \mathrm{Top}_{*} & \rightarrow \mathrm{Top}_{*} \\
X & \mapsto X \wedge \mathbb{S}^{N}
\end{aligned}
$$

Proposição 3.2.3. O par $\left(\Sigma^{N} \dashv \Omega^{N}\right)$ forma uma adjunção de Quillen.

Proof: Esse é um caso particular da proposição 2.2.2 já que $\mathbb{S}^{N}$ é cofibrante.

Para definir um espaço de laços infinito precisamos da categoria de espectros equipada com a estrutura modelo estável [BF78, Sc97].

Definição 3.2.4. Um espectro é uma sequencia de espaços topológicos pontuados $\left(X_{\bullet}\right)_{\bullet} \in \mathbb{N} \in$ $\prod_{\bullet \in \mathbb{N}} \mathrm{Top}_{*}$ equipados com aplicações estruturais $\left(\sigma_{\bullet}^{X}\right)_{\bullet \in \mathbb{N}} \in \prod_{\bullet \in \mathbb{N}} \mathrm{T}_{*}\left(X_{\bullet} \wedge \mathbb{S}^{1}, X_{\bullet+1}\right)$.

Uma aplicação de espectros é uma sequencia de aplicações pontuadas $\left(f_{\bullet}\right)_{\bullet \in \mathbb{N}} \in \prod_{\bullet \in \mathbb{N}} \operatorname{Top}_{*}\left(X_{\bullet}\right.$, $\left.Y_{\bullet}\right)$ tais que $\sigma_{\bullet}^{Y}\left(f_{\bullet} \wedge 1_{\mathbb{S}^{1}}\right)=f_{\bullet+1} \sigma_{\bullet}$.

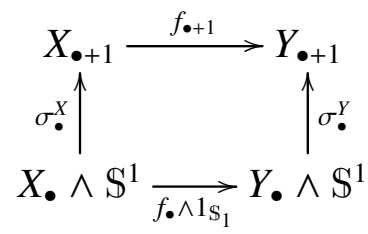

A categoria de espectros é denotada por $\mathrm{Sp}$.

Definição 3.2.5. Seja $q \in \mathbb{Z}$. O p-ésimo grupo de homotopia estável de um espectro é $\pi_{q}^{S}\left(X_{\bullet}\right):=$ $\operatorname{colim}_{\bullet \rightarrow \infty} \pi_{q+\bullet}\left(X_{\bullet}\right)$.

Um espectro $X_{\bullet}$ é conectivo se $\pi_{q}\left(X_{\bullet}\right)$ for trivial para todo $q<0$.

Um morfismo de espectros que induz um isomorfismo nos grupos de homotopia estáveis é uma equivalência homotópica fraca estável.

A categoria de espectros Sp é bicompleta e os limítes e colimites podem ser computados em cada índice, o que pode ser verificado checando as propriedades universais diretamente.

$\mathrm{O}$ funtor esquecimento $U: \mathrm{Sp} \rightarrow \prod_{\bullet \in \mathbb{N}} \mathrm{Top}$ admite um adjunto à esquerda

$$
\begin{aligned}
S: \prod_{\bullet \in \mathbb{N}} \mathrm{Top}_{*} & \rightarrow \mathrm{Sp} \\
X_{\bullet} & \mapsto \bigsqcup_{i=0}^{\bullet}\left(X_{i} \wedge \mathbb{S}^{\bullet-i}\right)
\end{aligned}
$$

Essa adjunção satisfaz as condições da proposição 1.4.7 já que $U$ preserva colimites, e porque $S$ manda uma fibração trivial geradora $j^{q}$ em uma sequência de fibrações triviais, já que $-\wedge \mathbb{S}^{\bullet}$ preserva cofibrações triviais. A estrutura modelo estrita $(\bar{W}, \bar{C}, \bar{F})$ é a estrutura modelo transferida 
pela adjunção $(S \dashv U)$, i.e. $f_{\bullet} \in \operatorname{Sp}\left(X_{\bullet}, Y_{\bullet}\right)$ é uma equivalência fraca se $f_{q} \in W_{Q}\left(X_{q}, Y_{q}\right)$ para cada $q \in \mathbb{N}$, é uma fibração se $f_{q} \in F_{Q}\left(X_{q}, Y_{q}\right)$ para cada $q \in \mathbb{N}$ e é uma cofibração se $f_{0} \in C_{Q}\left(X_{0}, Y_{0}\right)$ e $\left(f_{q+1}, \sigma_{q}^{Y}\right) \in C_{Q}\left(X_{q+1} \sqcup_{X_{q} \wedge \mathbb{S}^{1}} Y_{q} \wedge \mathbb{S}^{1}, Y_{q+1}\right)$ para cada $q \in \mathbb{N}$. Um espectro $X$. é cofibrante se $X_{0}$ for cofibrante e cada morfismo estrutural $\sigma_{q}^{X}$ for uma cofibração. Note que isso implica em particular que todos os espaços $X$. de um espectro cofibrante são cofibrantes. Denotamos a categoria de espectros equipada com a estrutura modelo estrita por $\overline{\mathrm{Sp}}$. Como a categoria $\mathrm{Top}$ * é própria e os pushouts e pullbacks são computados em cada índice é fácil ver que a estrutura modelo estrita é própria.

Defina o funtor

$$
\begin{aligned}
\Omega: \overline{\mathrm{Sp}} & \rightarrow \overline{\mathrm{Sp}} \\
X_{\bullet} & \mapsto \operatorname{colim}_{p \rightarrow \infty} \Omega^{p} X_{\bullet+p}
\end{aligned}
$$

Proposição 3.2.6. O funtor $\Omega$ equipado com a inclusão natural $I d_{S p} \Rightarrow \Omega$ é uma monada idempotente de Quillen tal que as $\Omega$-equivalências fracas são as equivalências homotópicas fracas estáveis.

Demonstração: Temos que Sp é próprio à direita. Provamos que $\Omega$ satisfaz as condições da definição 1.8.3. Temos que 1.8.3.i) e 1.8.3.v) são satisfeitas pois estamos assumindo que $=$ e $\eta^{\prime}$ são o funtor e a transformação natural de identidade. Como os funtores $\Omega^{p}$ preservam equivalências fracas $\Omega$ preserva equivalências fracas estritas, e portanto $\Omega$ satisfaz 1.8.3.ii). Temos que 1.8.3.iii) é satisfeita pois $\Omega \Omega=\Omega$. Note agora que como $U$ e cada $\Omega^{p}$ são adjuntos à direita eles preservam limites e portanto para todo diagrama de espectros $Z_{\bullet} \stackrel{h}{\rightarrow} Y_{\bullet} \stackrel{f}{\leftarrow} X_{\bullet}$ temos que $\Omega\left(Z_{\bullet} \times_{Y_{\bullet}} X_{\bullet}\right)=$ $\Omega_{\bullet} \times_{\Omega Y_{\bullet}} \Omega X_{\bullet}$. Então por $\Omega$ preservar fibrações, já que cada $\Omega^{q}$ preserva fibrações, e por Top ${ }_{*}$ ser próprio à direita temos que $\Omega$ satisfaz 1.8.3.iv). Que as $\Omega$-equivalências fracas são as equivalência homotópicas fracas estáveis segue do fato que os funtores de grupos de homotopia comutam com colimites filtrados e portanto

$$
\pi_{\bullet+q} \Omega X_{\bullet}=\pi_{\bullet+q} \operatorname{colim}_{p \rightarrow \infty} \Omega^{p} X_{\bullet+p}=\operatorname{colim}_{p \rightarrow \infty} \pi_{\bullet+q+p} X_{\bullet+p}=\pi_{q}^{S} X_{\bullet}
$$

A estrutura modelo estável $\left(W_{\Omega}, C_{\Omega}, F_{\Omega}\right)$ em Sp é dada pela localização de Bousfield da estrutura modelo estrita via a monada idempotente $\Omega$. Pela proposição 1.8 .8 uma aplicação $f_{\bullet} \in$ $F_{\Omega}\left(X_{\bullet}, Y_{\bullet}\right)$ se $f_{q} \in F_{Q}\left(X_{q}, Y_{q}\right)$ e $\left(\Omega^{1}\left(\sigma_{q}^{X}\right) \eta_{X_{q}}^{1}, f_{q}\right) \in W_{Q}\left(X_{q}, \Omega^{1} X_{q+1} \times_{\Omega^{1} Y_{q+1}} Y_{q}\right)$ para cada $q \in \mathbb{N}$. Denotamos a categoria de espectros equipada com a estrutura modelo estável por $\mathrm{Sp}$. Os espectros de interesse para a teoria de homotopia estável são os $\Omega$-espectros, que são os espectros tais que $\Omega^{1}\left(\sigma_{\bullet}\right) \eta_{X_{\bullet}}^{1} \in W\left(X_{\bullet}, \Omega^{1} X_{\bullet+1}\right)$. Os objetos fibrantes da estrutura modelo estável Sp são $\Omega$-espectros.

Na categoria Top temos que fibrações induzem sequencias exatas longas de grupos de homotopia. Essas sequências exatas longas induzem sequências exatas longas de grupos de homotopia estáveis de fibrações de espectros. A pesar de não termos sequências exatas longas de cofibrações 
em Top (a inclusão de $\mathbb{S}^{1}$ como o bordo de $I^{2}$ é um contra exemplo clássico) em $\mathrm{Sp}$ existem sequências exatas longas de grupos de homotopia estáveis. Enunciamos essa propriedade sem demonstração pois ela fugiria do escopo dessa tese. Ela pode ser encontrada em [MMSS01, T. 7.4].

Proposição 3.2.7. Seja $p_{\bullet} \in F_{\Omega}\left(E_{\bullet}, B_{\bullet}\right)$ e $F_{\bullet}$ o espectro de fibras de $p_{\bullet}$, i.e. $F_{\bullet}=E_{\bullet} \times_{B_{\bullet}} *$ Temos uma sequência exata longa de grupos de homotopia estáveis

$$
\cdots \rightarrow \pi_{q+1}^{S}\left(B_{\bullet}\right) \rightarrow \pi_{q}^{S}\left(F_{\bullet}\right) \rightarrow \pi_{q}^{S}\left(E_{\bullet}\right) \rightarrow \pi_{q}^{S}\left(B_{\bullet}\right) \rightarrow \pi_{q-1}^{S}\left(F_{\bullet}\right) \rightarrow \cdots
$$

Seja $\iota_{\bullet} \in C_{\Omega}\left(B_{\bullet}, Y_{\bullet}\right)$ e $C_{\bullet}$ o espectro de cofibras de $\iota_{\bullet}$, i.e. $C_{\bullet}=Y_{\bullet} \sqcup_{B_{\bullet}}$ *. Temos uma sequência exata longa de grupos de homotopia estáveis

$$
\cdots \rightarrow \pi_{q+1}^{S}\left(C_{\bullet}\right) \rightarrow \pi_{q}^{S}\left(B_{\bullet}\right) \rightarrow \pi_{q}^{S}\left(Y_{\bullet}\right) \rightarrow \pi_{q}^{S}\left(C_{\bullet}\right) \rightarrow \pi_{q-1}^{S}\left(B_{\bullet}\right) \rightarrow \cdots
$$

Temos que Sp é uma categoria modelo própria.

Proposição 3.2.8. A estrutura modelo estável de Sp é própria.

Demonstração: Pela proposição 1.8.9 essa estrutura modelo é própria à esquerda. Seja $p . \in$ $F_{\Omega}\left(E_{\bullet}, B_{\bullet}\right)$ e $f_{\bullet} \in W_{\Omega}\left(A_{\bullet}, B_{\bullet}\right)$. Seja $F_{\bullet}$ o espectro de fibras de $p_{\bullet}$. Temos que $F_{\bullet}$ é homeomorfo à fibra de $f_{\bullet}^{*} p$. Logo a sequência exata longa dos grupos de homotopia estáveis e o lema dos 5 nos dão que $p_{\bullet}^{*} f_{\bullet} \in W_{\Omega}$

Definição 3.2.9. O funtor de espaços de laços infinitos é

$$
\begin{aligned}
\Omega^{\infty}: \mathrm{Sp} & \rightarrow \mathrm{Top}_{*} \\
X_{\bullet} & \mapsto \operatorname{colim}_{\bullet \rightarrow \infty} \Omega^{\bullet} X_{\bullet}
\end{aligned}
$$

Este funtor admite um adjunto de Quillen à esquerda.

Definição 3.2.10. O funtor de espectros de suspensões infinitas é

$$
\begin{aligned}
\Sigma^{\infty}: \mathrm{Top}_{*} & \rightarrow \mathrm{Sp} \\
X & \mapsto \Sigma^{\bullet} X
\end{aligned}
$$

Proposição 3.2.11. O par $\left(\Sigma^{\infty} \dashv \Omega^{\infty}\right)$ forma uma adjunção de Quillen.

Demonstração: A adjunção é induzida das adjunções da proposição 3.2 .3 e por construção $\Sigma^{\infty}$ preserva cofibrações e cofibrações triviais. 


\subsection{2-Espaços de laços relativos e espectros relativos}

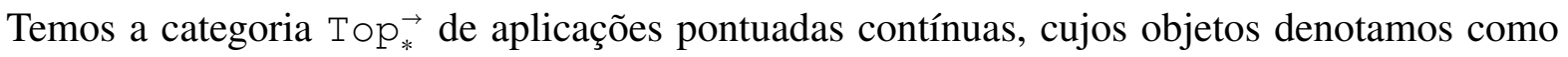
$\iota: B \rightarrow Y$, que podemos pensar como uma categoria de funtores da categoria $\rightarrow:=0 \rightarrow 1 \mathrm{com}$ apenas dois objetos e um morfismo não identidade entre eles. Como $\rightarrow$ é uma categoria de Reedy temos uma estrutura modelo $\left(W^{\rightarrow}, C^{\rightarrow}, F^{\rightarrow}\right)$ em $\operatorname{Top}_{*}^{\rightarrow}$. Seja $(k, l) \in \operatorname{Top}_{*}^{\rightarrow}\left(l, \iota^{\prime}\right)$. Temos $(k, l) \in W^{\rightarrow}$ se $k$ e $l$ são equivalências homotópicas fracas, $(k, l) \in F^{\rightarrow}$ se $k$ e $l$ forem fibrações de Serre e $(k, l) \in C^{\rightarrow}$ se $k$ e $\left(l, \iota^{\prime}\right) \in \operatorname{Top}_{*}\left(Y \sqcup_{B} B^{\prime}, Y^{\prime}\right)$ forem cofibrações. Nos referimos a Top $\rightarrow$ como a categoria de espaços relativos, pois os objetos cofibrantes dessa categoria são retrações de CWpares. Todos os objetos dessa categoria são fibrantes.

Definição 3.3.1. Um espaço relativo $\iota: B \rightarrow Y$ é $m$-conexo se $B$ é $m-1$-conexo e $Y$ é $m$-conexo.

Seja $N \in \mathbb{N}$ e $I$ o espaço pontuado $[0,1]$ com ponto base $I_{*}:=1$.

Definição 3.3.2. O funtor de espaços de $N$-laços relativos é

$$
\begin{aligned}
\Omega_{\mathrm{rel}}^{N}: \mathrm{Top}_{*} & \rightarrow \mathrm{Top}_{*} \\
\iota: B \rightarrow Y & \mapsto\left[\mathbb{S}^{N-1}, B \times_{Y}[I, Y]_{*}\right]_{*}
\end{aligned}
$$

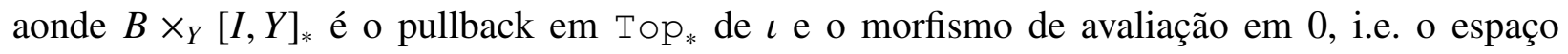
$\left\{(a, \beta) \in B \times[I, Y] \mid \beta(0)=\iota(a), \beta(1)=Y_{*}\right\}$. Essa construção é conhecida como a fibra homotópica de $\iota$.

Se $\iota: B \rightarrow Y$ for $m$-conexo então $B \times_{Y}[I, Y]_{*}$ é $m-1$-conexo.

Definição 3.3.3. O funtor de 2-espaços de N-laços é

$$
\begin{aligned}
\Omega_{2}^{N}: \operatorname{Top}_{N-1} & \rightarrow \mathrm{Top}_{*}^{2} \\
\iota: B \rightarrow Y & \mapsto\left(\Omega^{N} Y, \Omega_{\mathrm{rel}}^{N} \iota\right) .
\end{aligned}
$$

Esse funtor admite um adjunto de Quillen fraco à esquerda.

Definição 3.3.4. O funtor de espaços relativos de $N$-suspensões é

$$
\begin{aligned}
\Sigma_{2}^{N}: \operatorname{Top}_{*}^{2} & \rightarrow \operatorname{Top}_{N-1} \\
\left(X_{c}, X_{o}\right) & \mapsto\left(\begin{array}{c}
\Sigma^{N-1} X_{o} \\
{\left[x_{o}, s\right] \mapsto\left[\Sigma_{o}^{N-1}\left(X_{o} \wedge I\right) \vee \Sigma^{N} X_{c}\right.}
\end{array}\right)
\end{aligned}
$$

Note que para $f \in C(X, Y)$ no geral não é verdade que $\left(i_{0}^{Y}, f \wedge 1_{I}\right) \in \operatorname{Top}_{*}\left(Y \sqcup_{X} X \wedge I, Y \wedge I\right)$ é uma cofibração (essa aplicação é uma cofibração se $Y$ é cofibrante). Isso implica que $\Sigma_{2}^{N}$ não preserva cofibrações. Além disso $\Omega_{\text {rel }}^{N}$ não preserva fibrações, e portanto $\Omega_{2}^{N}$ também não. 
Proposição 3.3.5. Os pares $\left(\Sigma_{2}^{N} \dashv \Omega_{2}^{N}\right)$ formam adjunções de Quillen fracas.

Proof: A unidade da adjunção é a transformação natural $\eta_{2}^{N}: I d_{\mathrm{Top}_{*}^{2}} \Rightarrow \Omega_{2}^{N} \Sigma_{2}^{N}$ definida por

$$
\begin{aligned}
\eta_{c}^{N}\left(x_{c}\right)(t) & :=\left[x_{c}, t\right] ; \\
\eta_{o}^{N}\left(x_{o}\right)(s) & :=\left(\left[x_{o}, s\right], s^{\prime} \mapsto\left[x_{o}, s^{\prime}, s\right]\right) ;
\end{aligned}
$$

e a counidade é a transformação natural $\epsilon_{2}^{N}: \Sigma_{2}^{N} \Omega_{2}^{N} \Rightarrow I d_{\text {Top } \rightarrow}$ definida por

$$
\begin{aligned}
\epsilon_{\mathrm{Dom}}^{N}([(\alpha, \beta), s]) & :=\alpha(s) ; \\
\epsilon_{\text {Codom }}^{N}\left(\left[(\alpha, \beta), s^{\prime}, s\right]\right) & :=\beta(s)\left(s^{\prime}\right) ; \\
\epsilon_{\text {Codom }}^{N}([\gamma, t]) & :=\gamma(t) .
\end{aligned}
$$

É fácil checar que as equações de unidade-counidade são satisfeitas.

Como pela proposição 2.2.2 os funtores $\Sigma^{N-1}, \Sigma^{N} \mathrm{e}-\wedge I$ preservam cofibrações eles em particular preservam objetos cofibrantes, e assim como pela proposição 1.1.11 coprodutos de objetos cofibrantes é cofibrante, a imagem de $\Sigma_{2}^{N}$ em objetos cofibrantes é uma aplicação entre objetos cofibrantes. Além disso a inclusão de um objeto cofibrante na base do seu cone é uma cofibração. Portanto $\Sigma_{2}^{N}$ preserva objetos cofibrantes. A proposição 2.2.2 e o lema de Ken Brown 1.3.6 implicam que $\Sigma^{N}$ preserva equivalências fracas entre objetos cofibrantes e a proposição 1.1.11.v) e o lema de Ken Brown 1.3.6 implicam que equivalências fracas entre objetos cofibrantes são fechados por coprodutos, portanto $\Sigma_{2}^{N}$ preserva equivalências fracas entre objetos cofibrantes.

Claramente $\Omega_{2}^{N}$ preserva objetos fibrantes já que todos os objetos são fibrantes em Top $\mathrm{p}_{*}^{2}$. Para todo espaço relativo $\iota: B \rightarrow Y$ obtemos uma sequência exata natural de espaços pontuados

$$
\Omega^{N} B \rightarrow \Omega^{N} Y \rightarrow \Omega_{\mathrm{rel}}^{N} \iota \rightarrow \Omega^{N-1} B \rightarrow \Omega^{N-1} Y
$$

que induz uma sequencia exata de grupos de homotopia [Ma99, Ch 8.6] e portanto pelo fato que $\Omega^{N}$ preserva equivalências fracas e pelo lema dos cinco $\Omega_{\text {rel }}^{N}$ preserva equivalências fracas.

Note que $\Sigma_{2}^{N}$ preserva cofibrações e cofibrações triviais entre objetos cofibrantes. Podemos definir uma outra estrutura modelo na categoria de espaços relativos via a adjunção $\left(\Sigma_{2}^{N} \dashv \Omega_{2}^{N}\right)$.

Definição 3.3.6. Seja $m \in \mathbb{N}$ com $m>0$. Uma aplicação de espaços relativos $(k, l) \in \mathrm{Top}_{*}(\iota$ : $B \rightarrow Y, \iota^{\prime}: B^{\prime} \rightarrow Y^{\prime}$ ) é uma $m$-1-equivalência homotópica relativa fraca se os homomorfismos induzidos $l_{*} \in \operatorname{Grp}\left(\pi_{q} Y, \pi_{q} Y^{\prime}\right)$ são isomorfismos para todo $q \geq m$ e se os homomorfismos induzidos $\left(k,\left[1_{I}, l\right]_{*}\right)_{*} \in \operatorname{Grp}\left(\pi_{q}\left(B \times_{Y}[I, Y]_{*}\right), \pi_{q}\left(B^{\prime} \times_{Y^{\prime}}\left[I, Y^{\prime}\right]_{*}\right)\right)$ são isomorfismos para todo $q \geq m-1$.

Proposição 3.3.7. A adjunção $\left(\Sigma_{2}^{N} \dashv \Omega_{2}^{N}\right)$ induz uma estrutura modelo $\left(W_{N-1}^{\rightarrow}, C_{N-1}^{\rightarrow}, F_{N-1}\right)$ em Top $_{*}$ cujos objetos cofibrantes são retrações de CW-pares $N-1$-conexos e cuja classe de equivalências fracas $W_{N-1}^{\rightarrow}$ são as $N$ - 1-equivalência homotópicas relativas fracas. 
Demonstração: Temos que a estrutura modelo de $\mathrm{Top}_{*}^{2}$ é gerada pela cofibrações geradoras $I_{*}^{2}:=1_{*} \times\left(I_{Q} \sqcup *\right) \amalg\left(I_{Q} \sqcup *\right) \times 1_{*}$ e cofibrações triviais geradoras $J_{*}^{2}:=1_{*} \times\left(J_{Q} \sqcup *\right) \amalg\left(J_{Q} \sqcup *\right) \times$ $1_{*}$. Assim é fácil ver que os domínios das imagens por $\Sigma_{2}^{N}$ das cofibrações e cofibrações triviais geradoras são compactos, e portante pequenos. Além disso como $\Sigma_{2}^{N}$ preserva cofibrações triviais entre objetos cofibrantes e $\Omega^{N}$ preserva equivalências fracas temos que $\Omega_{2}^{N} \operatorname{Cel}\left(\Sigma_{2}^{N} J_{*}^{2}\right) \subset W_{Q}^{2}$. Logo essa adjunção satisfaz as condições do teorema 1.4.7.

Como todo espaço é fibrante na estrutura modelo de Quillen os espaços relativos também vão ser fibrantes na estrutura modelo transferida. Claramente os espaços relativos cofibrantes na estrutura modelo transferida são $\mathrm{CW}$-complexos relativos $N-1$-conexos pois os domínios são obtidos colando $q+N-1$-células e os codomínios são obtidos colando $q+N$-células e cones de $q+N-1$-células. Que $\Omega_{2}^{N}(k, l) \in W^{\rightarrow}$ se e somente se $(k, l) \in W_{N-1}^{\rightarrow}$ segue das definições e do fato que para todo espaço pontuado $X$ temos $\pi_{q} \Omega^{N} X=\pi_{q+N} X$.

Definição 3.3.8. Nos referimos à estrutura modelo transferida da proposição acima $\left(W_{N-1}, C_{N-1}\right.$, $F_{N-1}$ ) como a estrutura relativa $N$ - 1-conexa e denotamos a categoria de espaços relativos equipada com ela por $\operatorname{Top}_{N-1}$.

Proposição 3.3.9. Temos que $(I d \dashv I d): T o p_{N-1} \rightleftharpoons T o p_{*}^{\rightarrow}$ é uma adjunção de Quillen fraca que induz a inclusão da subcategoria homotópica coreflexiva de espaços relativos $N-1$-conexos.

Demonstração: Trivialmente o funtor $I d: \mathrm{Top}_{*} \rightarrow \mathrm{Top}_{N-1}$ preserva objetos fibrantes e equivalências fracas. Temos $\Sigma_{2}^{N}\left(I_{*}^{2}\right) \subset C^{\rightarrow}$ e toda $N-1$-equivalência homotópica relativa fraca entre espaços relativos $N$-1-conexos é uma equivalência homotópica relativa fraca. Logo (Id Id) $: \operatorname{Top}_{N-1} \rightleftharpoons \mathrm{Top}_{*}$ é uma adjunção de Quillen fraca.

Pelo argumento anterior todo objeto cilíndrico em objetos cofibrantes de $\mathrm{T} \circ \mathrm{p}_{N-1}$ é um objeto cilíndrico em $\mathrm{Top}_{*}^{\rightarrow}$ e portanto as relações de homotopia coincidem. Logo a inclusão da subcategoria homotópica é plena.

Definimos agora a categoria de espectros relativos e definimos o funtor de espaços de laços relativos infinitos.

Definição 3.3.10. Um espectro relativo é um par de espectros $B_{\bullet}$ e $Y_{\bullet}$ equipados com uma sequências de aplicações pontuadas $\left(\iota_{\bullet}\right)_{\bullet \in \mathbb{N}} \in \prod_{\bullet \in \mathbb{N}} \operatorname{Top}_{*}\left(B_{\bullet}, Y_{\bullet+1}\right)$ tais que $\sigma_{\bullet+1}^{Y}\left(\iota_{\bullet}, 1_{\mathbb{S}^{1}}\right)=\iota_{\bullet+1} \sigma_{\bullet}^{B}$. No geral denotamos espectros relativos simplesmente por $\iota_{\bullet}$.

Uma aplicação de espectros relativos é um par de aplicações de espectros $\left(\left(k_{\bullet}\right)_{\bullet \in \mathbb{N}},\left(l_{\bullet}\right)_{\bullet \in \mathbb{N}}\right)$ tais que $l_{q+1} \iota_{q}=\iota_{q}^{\prime} k_{q}$ para cada $q \in \mathbb{N}$. Denotamos a categoria de espectros relativos por $\mathrm{Sp}^{\nearrow}$.

A categoria de espectros relativos $\mathrm{Sp}^{\nearrow}$ é bicompleta e os limites e colimites podem ser computados em cada índice.

Definição 3.3.11. Um espectro relativo é conectivo se o par de espectros subjacentes forem conectivos. 
$\mathrm{O}$ funtor esquecimento $U: \mathrm{Sp}^{\nearrow} \rightarrow \mathrm{Sp}^{2}$ admite um adjunto à esquerda:

$$
\begin{aligned}
& S: \mathrm{Sp}^{2} \rightarrow \mathrm{Sp}^{\nearrow} \\
& \left(B_{\bullet}, Y_{\bullet}\right) \mapsto\left(i_{B_{\bullet}}: B_{\bullet} \rightarrow B_{\bullet} \vee Y_{\bullet+1}\right)
\end{aligned}
$$

aonde fixamos $B_{-1} \vee Y_{0}=Y_{0}$ e os morfismos estruturais $\sigma_{\bullet} \in \mathrm{Top}_{*}\left(\left(B_{\bullet-1} \vee Y_{\bullet}\right) \wedge \mathbb{S}^{1}, B \bullet \vee Y_{\bullet+1}\right)$ são induzidos dos morfismos estruturais de $B_{\bullet}$ e $Y_{\bullet}$ usando o fato que $-\wedge \mathbb{S}^{1}$ preserva colimites.

A estrutura modelo estável $\left(W_{\Omega^{\nearrow}}, C_{\Omega^{\nearrow}}, F_{\Omega^{\nearrow}}\right)$ em $\mathrm{Sp}^{\nearrow}$ é a estrutura modelo transferida da estrutura modelo estável em $\mathrm{Sp}^{2}$ pela adjunção $(U \dashv S)$, que existe pela proposição 1.4.7 já que $U$ preserva colimites e $S$ manda cofibrações triviais em pares de equivalências fracas estritas. Os objetos fibrantes são os espectros relativos cujos espectros subjacentes são $\Omega$-espectros e os objetos cofibrantes são os espectros relativos cujos espectros subjacentes são cofibrantes e tais que os $\iota$. são cofibrações.

Definição 3.3.12. O funtor de 2-espaços de laços infinitos é

$$
\begin{aligned}
\Omega_{2}^{\infty}: \mathrm{Sp}^{\rightarrow} & \rightarrow \mathrm{Top}_{*}^{2} \\
\iota_{\bullet}: B_{\bullet} & \rightarrow Y_{\bullet+1} \mapsto\left(\lim ^{\bullet} \Omega^{\bullet} Y_{\bullet}, \underline{\lim } \Omega_{\mathrm{rel}}^{\bullet} \iota_{\bullet}\right) .
\end{aligned}
$$

Definição 3.3.13. O funtor de espectros relativos de suspensões infinitas é

$$
\begin{aligned}
\Sigma_{2}^{\infty}: \mathrm{Top}_{*}^{2} & \rightarrow \mathrm{Sp}^{\nearrow} \\
\left(X_{c}, X_{o}\right) & \mapsto\left(\begin{array}{c}
\Sigma^{\bullet} X_{o} \\
{\left[x_{o}, s\right] \mapsto\left[\Sigma^{\bullet}\left(X_{o} \wedge I\right) \vee \Sigma^{\bullet+1} X_{c}\right)}
\end{array}\right)
\end{aligned}
$$

aonde fixamos $\Sigma^{-1}\left(X_{o} \wedge I\right) \vee \Sigma^{0} X_{c}=X_{c}$.

Corolário 3.3.14. O par $\left(\Sigma_{2}^{\infty} \dashv \Omega_{2}^{\infty}\right)$ forma uma adjunção de Quillen fraca.

Demonstração Segue da proposição 3.3.5.

\subsection{Realização geométrica}

O funtor de realização geométrica nos dá um espaço a partir de um espaço simplicial. Essa construção é um caso particular de um coend. Seja $\Delta^{\langle\bullet\rangle} \in \mathrm{Top} \mathrm{p}^{\Delta}$ o espaço cosimplicial de simplexos dado por

$$
\begin{aligned}
\Delta^{-}: \Delta & \rightarrow \text { Top } \\
\langle q\rangle & \mapsto\left\{\left(u^{i}\right)_{i \in\langle q\rangle} \in \mathbb{R}^{\langle q\rangle} \mid \forall i \in\langle q\rangle, x^{i} \geq 0 \text { e } \sum_{i=1}^{q} x^{i}=1\right\}
\end{aligned}
$$


Definição 3.4.1. O funtor de realização geométrica em Top é definido por:

$$
\begin{aligned}
|-|: \mathrm{Top}^{\Delta^{\mathrm{op}}} & \rightarrow \mathrm{Top} \\
X^{\langle\bullet\rangle} & \mapsto \int^{\Delta} X^{\langle q\rangle} \times \Delta^{\langle q\rangle}
\end{aligned}
$$

O funtor de realização geométrica é adjunto à esquerda do funtor de espaços de complexos singulares

$$
\begin{aligned}
\text { Sing : Top } & \rightarrow \mathrm{Top}^{\Delta^{\mathrm{op}}} \\
X & \mapsto\left[\Delta^{\langle\bullet\rangle}, X\right],
\end{aligned}
$$

e como $\Delta^{-} \in \mathrm{Top}_{\text {Cof }}^{\Delta}$ a proposição 2.2.8 implica que essa adjunção é de Quillen. Propriedades básicas dessa construção podem ser encontradas em [Ma72, C.11].

Esse funtor induz uma realização geométrica em $\mathrm{Top}_{*}, \mathrm{Top}_{*}^{2}, \mathrm{Top}_{*}^{\rightarrow}, \mathrm{Sp}$ e $\mathrm{Sp}^{\rightarrow}$. Denotamos por $B(F, C, X)$ a realização geométrica $|B .(F, C, X)|$ nos casos em que $F$ é um $C$-funtor em $\mathcal{A}$ $\operatorname{com} \mathcal{A}$ qualquer uma dessas categorias. De $[\mathrm{Ma} 72,9.2,11.8]$ temos que qualquer aplicação $f \in$ $\mathcal{T}(Y, F X)$ determina uma aplicação $\tau(f) \in \mathcal{T}(Y, B(F, C, X))$ e qualquer aplicação $g \in \mathcal{T}(F X, Y)$ tal que $g \partial_{0}=g \partial_{1} \in \mathcal{T}(F C X, Y)$ determina uma aplicação $\varepsilon(g) \in \mathcal{T}(B(F, C, X), Y)$. Aplicações desse tipo são centrais no teorema de reconhecimento relativo, tanto o original quanto o relativo do capítulo 5 . 



\section{Capítulo 4}

\section{Operads e 2-operads}

Nesse capítulo apresentamos a teoria de operads e de 2-operads, que são operads coloridos em duas cores linearmente ordenadas $\{c>o\}$. Existem diversas variações de operads, e aqui sempre usaremos operads e 2-operads simétricos reduzidos como na definição original de May em [Ma72]. Um operad $\mathcal{P}$ é uma sequência de objetos $\mathcal{P}(\underline{n}) \in \prod_{n \in \mathbb{N}} \mathcal{T}$ de uma categoria monoidal simétrica $\mathcal{T}$ equipados com ações dos grupos de simetria $\mathbb{S}_{\underline{n}}$ e $\operatorname{com} \mathcal{P}(\underline{0})=*$ que podem ser interpretados como objetos de operações abstratas, equipados com morfismos de composição entre eles que satisfazem condições de associatividade, unidade e equivariância. Um operad $\mathcal{P}$ define uma monada $P$ cujas álgebras são realizações concretas das operações abstratas em $\mathcal{P}$, no sentido que uma $P$-álgebra é um objeto $X \in \mathcal{T}_{*}$ equipado com morfismos $\mathcal{P}(\underline{n}) \otimes X^{\otimes \underline{n}} \rightarrow X$ compatíveis com a estrutura de operad de $\mathcal{P}$ tais que o ponto base age como um elemento neutro. Um 2-operad $Q$ é composto por uma sequência de objetos $Q(\underline{n}) \in \prod_{n \in \mathbb{N}} \mathcal{T}$ equipados com ações de $\mathbb{S}_{\underline{n}}$ e uma bisequência de objetos $Q(\underline{n, m}) \in \prod_{(n, m) \in \mathbb{N}^{2}} \mathcal{T}$ equipados com ações de $\mathbb{S}_{\underline{n}} \times \mathbb{S}_{\underline{m}} \operatorname{com} Q(\underline{0})=*=Q(\underline{0,0})$ equipados com morfismos de composição entre eles que satisfazem condições de associatividade, unidade e equivariância. Um 2-operad $Q$ define uma monada $Q$ em $\mathcal{T}_{*}^{2}$ tal que uma $Q$-álgebra é um par $\left(X_{c}, X_{o}\right) \in \mathcal{T}_{*}^{2}$ equipado com morfismos $Q(\underline{n}) \otimes X_{c}^{\otimes n} \rightarrow X_{c}$ e $Q(\underline{n, m}) \otimes X_{c}^{\otimes n} \otimes X_{o}^{\otimes m} \rightarrow X_{o}$ compatíveis com a estrutura de 2-operad de $Q$. Em categorias modelo monoidais simétricas satisfazendo certas condições a categoria de operads, 2-operads e de suas álgebras admitem uma estrutura modelo, e portanto noções da teoria de homotopia abstrata do capítulo 1 são aplicáveis nessas categorias.

Na primeira sessão apresentamos a definição de operads em uma categoria monoidal simétrica $\mathcal{T}$ como álgebras de uma monada definida por árvores na categoria de sequências simétricas em $\mathcal{T}$ e mostramos como construir suas monadas associadas via coends. Damos como exemplos de operads o operad dos monoides topológicos, o operad dos monoides comutativos topológicos e os operads dos pequenos $N$-cubos, e mostramos que espaços de laços são álgebras sobre esses operads. Também apresentamos os operads de Fulton-MacPherson e o operad de Barratt-Eccles.

Na segunda sessão apresentamos a definição de 2-operads em uma categoria monoidal simétrica $\mathcal{T}$ como álgebras de uma monada definida por árvores com arestas coloridas em $\{c>o\}$ na categoria de sequências simétricas coloridas em $\mathcal{T}$ e mostramos como construir suas monadas associadas via coends. Damos como exemplos de 2-operads o 2-operad das ações de monoides topológicos 
em espaços pontuados, o 2-operad dos homomorfismos de monoides comutativos topológicos e os 2-operads dos $N$-queijos suíços, e mostramos que 2-espaços de laços são álgebras sobre esses 2-operads. Apresentamos versões coloridas dos operads de Fulton-MacPherson, e também introduzimos uma versão colorida do operad de Barratt-Eccles.

$\mathrm{Na}$ terceira sessão descrevemos as condições para a existência de uma estrutura modelo na categoria de 2-operads dada em [BM07]. Introduzimos também a definição de $2 E_{\infty}$-operads que são 2-operads cofibrantes cujos espaços subjacentes são todos contráteis, ou equivalentemente que são resoluções cofibrantes do 2-operad de homomorfismos de monoides comutativos. Também apresentamos a estrutura modelo de álgebras sobre 2-operads e condições em que a construção bar em monadas de 2-operads cofibrantes preservam objetos cofibrantes e equivalências fracas entre eles.

$\mathrm{Na}$ quarta sessão apresentamos a construção das resoluções de Boardman-Vogt como descritas em [BV68, BM06] e usamos ela para provar que as resoluções dos 2-operads de FultonMacPherson são resoluções cofibrantes dos 2-operads dos queijos-suíssos. Outra consequência é que a resolução do 2-operad de Barratt-Eccles é um $2 E_{\infty}$-operad.

\subsection{Operads}

Definição 4.1.1. Seja $\mathbb{S}_{\text {inj }}$ a subcategoria de Set cujos objetos são os conjuntos $\underline{n}:=\{1, \ldots, n\}$ para cada $n \in \mathbb{N}, \operatorname{com} \underline{0}=\emptyset$, e cujos morfismos são as injeções.

Seja $\mathbb{S}$ a subcategoria de $\mathbb{S}_{\text {inj }}$ com os mesmos objetos e com as bijeções como morfismos.

Para cada $n \in \mathbb{N}$ o grupo de bijeções $\mathbb{S}_{\underline{n}}:=\mathbb{S}(\underline{n}, \underline{n})$ é isomorfo ao grupo de simetrias de $n$ elementos.

Definição 4.1.2. Seja $\mathcal{T}$ uma categoria monoidal simétrica bicompleta. Um $\mathbb{S}$-objeto em $\mathcal{T}$ é um funtor $\mathcal{P} \in \mathcal{T}^{\mathbb{S}^{\text {op }}}$ tal que $\mathcal{P}$ é reduzido, no sentido que $\mathcal{P}(\underline{0})=*$. Denotamos a categoria de $\mathbb{S}$-objetos em $\mathcal{T}$ por $\mathbb{S}-\mathcal{T}$.

Para cada $\mathcal{P} \in \mathbb{S}-\mathcal{T}$ e $n \in \mathbb{N}$ temos uma ação de $\mathbb{S}_{\underline{n}} \operatorname{em} \mathcal{P}(\underline{n})$.

Podemos usar árvores para definir uma monada na categoria de $\mathbb{S}$-objetos.

Definição 4.1.3. Seja $n>0$. Uma árvore enraizada orientada $T$ sobre $\underline{n}$ é um par de conjuntos finitos $V_{T}, E_{T} \in \operatorname{Set}_{\text {fin }}$, com $V_{T}$ os vértices internos e $E_{T}$ as arestas da árvore $T$, equipados com uma bijeção $s \in \operatorname{Set}\left(E_{T}, V_{T} \amalg \underline{n}\right)$ e uma função $t \in \operatorname{Set}\left(E_{T}, V_{T} \amalg\left\{v_{0}\right\}\right)$ tais que $t^{-1}\left(v_{0}\right)$ contenha exatamente um elemento, que chamamos de raiz e denotamos por $e_{0}$, e tais que para todo $v \in V_{T} \amalg \underline{n}$ existe um único caminho direcionado de arestas entre $v$ e $v_{0}$, i.e. uma sequencia $e^{\underline{k}} \in \prod_{i \in \underline{k}} E_{T}$, tal que $t e^{i}=s e^{i+1}$ para $i \in \underline{k-1}, s e^{1}=v$ e $t e^{k}=v_{0}$. Essa última condição induz uma ordem parcial em $E_{T}$ na qual $e \geq e^{\prime}$ se $e^{\prime}$ está contido no caminho entre $s(e)$ e $v_{0}$. Nos referimos aos elementos $\underline{n}$ como as folhas da árvore. 
Existe exatamente uma árvore sem vértices internos, que é a árvore em $\underline{1}$ com uma aresta, que chamamos de a árvore unitária.

Chamamos a única árvore sobre $\underline{n}$ contendo apenas um vértice interno como a $\underline{n}$-corola.

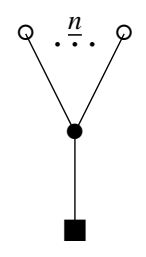

Usaremos a convenção que a única árvore enraizada sobre 0 é a única árvore com apenas um vértice interno e apenas uma aresta, a $\underline{0}$-corola. Essa condição é necessária para que a monada resultante seja reduzida.

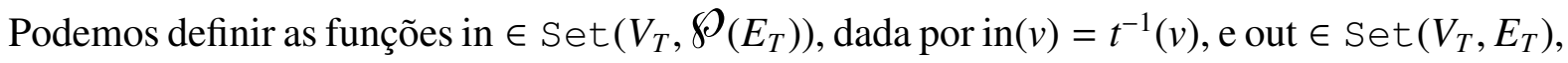
dada por out $(v)=s^{-1}(v)$. Note que in $(v)$ pode ser vazio. Definimos a função pre $\in \operatorname{Set}\left(V_{T}, V_{T} \amalg\left\{v_{0}\right\}\right)$ por $\operatorname{pre}(v)=t(\operatorname{out}(v))$.

Uma ordem em $T$ é uma ordem linear em $V_{T} \amalg \underline{n}$ tal que se out $(v)<\operatorname{out}\left(v^{\prime}\right)$ então $v<v^{\prime}$. Note uma ordem em $T$ induz uma ordem linear em in $(v)$ para cada $v \in V_{T}$.

Denotamos por $\mathbb{T}(\underline{n})$ a categoria cujos objetos são classes de isomorfismos de árvores enraizadas orientadas sobre $\underline{n}$ equipadas com uma ordem e cujos morfismos $\phi \in \mathbb{T}(\underline{n})\left(T, T^{\prime}\right)$ são pares de bijeções $\left(\phi_{V}, \phi_{E}\right) \in \operatorname{Set}\left(V_{T}, V_{T^{\prime}}\right) \times \operatorname{Set}\left(E_{T}, E_{T^{\prime}}\right)$ tais que $s^{\prime} \phi_{E}=\phi_{V} s$ e $t^{\prime} \phi_{E}=\phi_{V} t$, i.e. isomorfismos de árvores que não necessariamente preservam a ordem.

Há uma operação de enxerto de árvores. Seja $T_{0} \in \mathbb{T}(\underline{n})$ e $T_{v} \in \mathbb{T}(|\operatorname{in}(v)|)$ para cada $v \in V_{T_{0}}$. Defina $T_{0}\left[\left\{T_{v}\right\}_{v \in V_{T_{0}}}\right] \in \mathbb{T}(\underline{n})$ como a árvore obtida da união das árvores $T_{v}$ pela identificação da raíz de cada $T_{v}$ com a aresta $s^{-1}(\operatorname{out}(v))$ de $T_{\operatorname{pre}(v)}$. Se $T_{0}$ e cada $T_{v}$ forem ordenadas então $T_{0}\left[\left\{T_{v}\right\}_{v \in V_{T_{0}}}\right]$ herda uma ordem.

Podemos construir uma monada em $\mathbb{S}-\mathcal{T}$ usando coends.

Todo $\mathcal{P} \in \mathbb{S}-\mathcal{T}$ e $n \in \mathbb{N}$ induzem um funtor

$$
\begin{aligned}
\mathcal{P}: \mathbb{T}(\underline{n})^{\mathrm{op}} & \rightarrow \mathcal{T} \\
T & \mapsto \bigotimes_{v \in V_{T}} \mathcal{P}(\underline{|\operatorname{in}(v)|)}
\end{aligned}
$$


Para $\underline{n}=\underline{1}$ o somando associado à árvore unitária é a unidade da estrutura monoidal 1 . Os morfismos em $\mathbb{T}(\underline{n})^{\text {op }}$ trocam a ordem do produto tensorial e agem em cada fator $\mathcal{P}(|\operatorname{in}(v)|)$ via a permutação na ordem de in( $v)$. Na construção do coend a seguir, assumimos que o funtor é constante no fator covariante.

Definição 4.1.4. Para $\mathcal{P} \in \mathbb{S}-\mathcal{T}$ defina o $\mathbb{S}$-objeto

$$
\begin{aligned}
\mathbb{T} \mathcal{P}: \mathbb{S}^{\mathrm{op}} & \rightarrow \mathcal{T} \\
\underline{n} & \mapsto \int^{\mathbb{T}(\underline{n})} \mathcal{P}(T)
\end{aligned}
$$

As $\mathbb{S}_{\underline{n}}$-ações são dadas por ações nas folhas das árvores.

Esta construção define um endofuntor em $\mathbb{S}-\mathcal{T}$ que é o funtor subjacente de uma monada. A unidade $\eta_{\mathcal{P}}^{\mathbb{T}}: \mathcal{P} \rightarrow \mathbb{T} \mathcal{P}$ é a inclusão natural de $\mathcal{P}(\underline{n}) \rightarrow \mathbb{T} \mathcal{P}(\underline{n})$ no somando indexado pela $\underline{n}$-corola. O produto $\mu_{\mathcal{P}}^{\mathbb{T}}: \mathbb{T} \mathbb{T} \mathscr{P} \rightarrow \mathbb{T} \mathcal{P}$ é definido pela operação de enxerto.

Apontamos que em algumas apresentações, por exemplo [BM03, BM06], a construção do operad livre é feita sobre árvores que não estão equipadas com uma bijeção das folhas com os elementos de $\underline{n}$, e por isso na definição do operad livre de um $\mathbb{S}$-objeto aparece um fator $-\otimes\left[\mathbb{S}_{\underline{n}}\right] \mathbb{1}$ na construção do coend. As duas construções são equivalentes.

Definição 4.1.5. Um operad $\mathcal{P}$ em $\mathcal{T}$ é uma $\mathbb{T}$-álgebra. Denotamos a categoria de operads em $\mathcal{T}$ por $O p(\mathcal{T})$.

A estrutura de operad de $\mathcal{P}$ é completamente definida por morfismos

$$
\eta^{\mathcal{P}} \in \mathcal{T}(\mathbb{1}, \mathcal{P}(\underline{1}))
$$

que é a composição da inclusão do somando $\mathbb{1}$ de $\mathbb{T} P(\underline{1})$ associado à árvore sem vértices internos com a aplicação estrutural de $\mathbb{T}$-álgebra de $\mathcal{P}$, e, para cada $n \in \mathbb{N}$ e $\left(n^{i}\right)_{i \in \underline{n}} \in \mathbb{N}^{\underline{n}}$, um morfismo

$$
\mu_{\underline{n}, \underline{\left(\underline{n}^{i}\right) i \in \underline{\underline{n}}}}^{\mathcal{P}} \in \mathcal{T}\left(\mathcal{P}(\underline{n}) \otimes\left(\bigotimes_{i \in \underline{n}} \mathcal{P}\left(\underline{\left.n^{i}\right)}\right), \mathcal{P}\left(\sum_{i=1}^{n} n^{i}\right)\right)\right.
$$

dado pelo morfismo estrutural de $\mathbb{T}$-álgebra de $\mathcal{P}$ aplicado no somando indexado pela árvore obtida

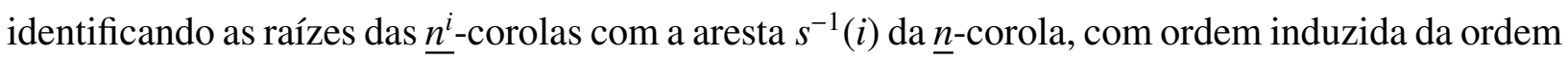
de $\underline{n}$.

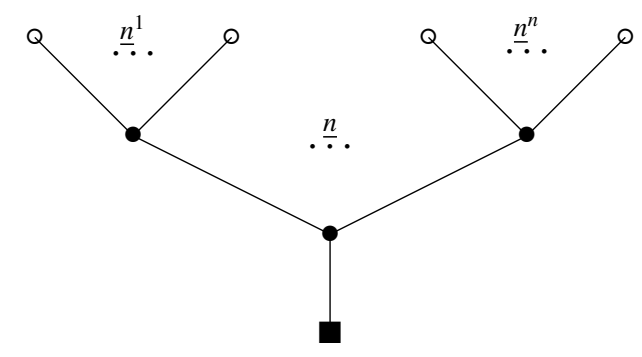


Esses morfismos satisfazem leis de associatividade, unidade e equivariância.

Todo operad $\mathcal{P}$ em $\mathcal{T}$ define uma monada em $\mathcal{T}_{*}$ da seguinte forma. Podemos estender o funtor subjacente de $\mathcal{P}$ em um funtor em $\mathbb{S}_{\text {inj }}^{\text {op }}$. Para cada injeção $v: \underline{n} \hookrightarrow \underline{n}^{\prime}$ podemos construir uma árvore substituindo as folhas da $\underline{n}^{\prime}$-corola que não estão na imagem de $v$ por vértices interiores. Isso resulta numa árvore sobre $\underline{n}$ e a estrutura de operad nos dá um morfismo $\mathcal{P}\left(\underline{n^{\prime}}\right) \rightarrow \mathcal{P}(\underline{n})$. Nos referimos a esses morfismos como as degenerações de $\mathcal{P}$.

Todo $X \in \mathcal{T}_{*}$ define um funtor

$$
\begin{aligned}
X^{\otimes-}: \mathbb{S}_{\text {inj }} & \rightarrow \mathcal{T} \\
\underline{n} & \mapsto X^{\otimes \underline{n}}
\end{aligned}
$$

com os morfismos induzidos pelas injeções trocando os índices das coordenadas e inserindo o ponto base $X_{*}$ nas coordenadas que não estão contidas na imagem via a composição $X_{*} *_{\mathbb{1}} \in$ $\mathcal{T}(\mathbb{1}, X)$.

Podemos então definir para todo operad $\mathcal{P}$ em $\mathcal{T}$ o endofuntor

$$
\begin{aligned}
P: \mathcal{T}_{*} & \rightarrow \mathcal{T}_{*} \\
X & \mapsto \int^{\mathbb{S}_{\mathrm{inj}}} \mathcal{P}(\underline{n}) \otimes X^{\otimes \underline{n}}
\end{aligned}
$$

com o ponto base dado pela inclusão de $\mathcal{P}(\underline{0}) \otimes X^{\otimes \underline{0}}=* \otimes \mathbb{1} \cong *$.

Esse endofuntor admite uma estrutura de monada com a unidade dada por $\eta^{\mathcal{P}} \otimes 1_{X} \in \mathcal{T}_{*}(X, \mathcal{P} X)$ e a multiplicação dada por $\prod_{\underline{n} \in \mathbb{N},\left(\underline{n}^{i}\right)_{i \in \underline{n}} \in \mathbb{N}^{\underline{n}} \underline{\underline{n}}} \mu_{\underline{n},\left(\underline{n^{i}}\right)_{i \in \underline{n}}}^{\mathcal{P}} \otimes 1_{X^{\otimes \sum_{i \in \underline{n}} n^{i}}} \in \mathcal{T}_{*}(\mathcal{P P} X, \mathcal{P} X)$.

Definição 4.1.6. Para um operad $\mathcal{P}$ uma $\mathcal{P}$-álgebra é uma $P$-álgebra para a monada associada $P$. Denotamos a categoria de $\mathcal{P}$-álgebra por $\mathcal{P}[\mathcal{T}]$.

Para todo $\mathcal{P} \in O p(\mathcal{T})$ e $X \in \mathcal{T}_{*}$ o objeto pontuado $P X$ admite uma filtração natural. Seja $F^{k} \mathbb{S}_{\text {inj }}$ a subcategoria plena de $\mathbb{S}_{\text {inj }}$ contendo apenas os conjunto $\underline{n}$ com $n \leq k$. Podemos definir $F^{k} P X$ como a imagem da inclusão natural de

$$
\int^{F^{k} \mathbb{S}_{\text {inj }}} \mathcal{P}(\underline{n}) \otimes X^{\otimes \underline{n}}
$$

em $P(X)$.

Damos agora alguns exemplos de operads em Top. O operad mais simples é o operad Com cujo $\mathbb{S}$-espaço subjacente é dado por $\operatorname{Com}(\underline{n}):=*$ para todo $n \in \mathbb{N}$. Os morfismos estruturais são óbvios dado que $*$ é o objeto terminal de Top. As Com-álgebras são os monoides comutativos topológicos, com a comutatividade dada pela trivialidade das $\mathbb{S}_{\underline{n}}$-ações. Note que esse é o objeto terminal de $O p(\mathcal{T})$. 
Também temos o operad Ass cujo $\mathbb{S}$-espaço subjacente é dado por $A s s(\underline{n}):=\mathbb{S}_{\underline{n}}$ para todo $n \in \mathbb{N}$ e com as $\mathbb{S}_{\underline{n}}$-ações dadas pela ação do grupo de simetrias em si mesmo. Os morfismos estruturais $\mu^{\text {Ass }}$ aplicados em $\left(\sigma,\left(\sigma^{j}\right)_{j \in n}\right)$ comutam blocos de elementos segundo $\sigma$ e comutam os elementos de cada bloco segundo cada $\sigma^{j}$, e são definidos pela seguinte fórmula:

$$
\mu_{\left.\underline{n}, \underline{n}^{j}\right)_{j \in \underline{n}}^{\mathrm{Ass}}}\left(\sigma,\left(\sigma^{j}\right)_{j \in \underline{n}}\right)(b):=\sigma^{j}\left(b-\sum_{l=1}^{j-1} n^{l}\right)+\sum_{l=1}^{\sigma(j)-1} n^{\sigma(l)}, \text { se } \sum_{l=1}^{j-1} n^{l}<b \leq \sum_{l=1}^{j} n^{l}
$$

As degenerações deletam os elementos que não estão na imagem. As Ass-álgebras são os monoides topológicos.

Seja $N \in \mathbb{N}$ e $M_{N \times N}^{\text {dgnl }}\left(\mathbb{R}^{+}\right)$o espaço de matrizes $N \times N$ diagonais com entradas reais positivas. O operad dos pequenos $N$-cubos $C^{N}$ é dado pelo $\mathbb{S}$-espaço

$$
C^{N}(\underline{n}):=\left\{\begin{array}{l|l}
d_{\underline{n}}:=\sqcup_{b \in \underline{n}} d_{b} \in\left[\sqcup_{b \in \underline{n}} I^{N}, I^{N}\right] & \begin{array}{l}
\exists M_{b} \in M_{N \times N}^{\mathrm{dgnl}}\left(\mathbb{R}^{+}\right), C_{b} \in I^{N} \text { t.q. } d_{b}(t)=M_{b} t+C_{b} ; \\
d_{b}\left(I^{N}\right) \cap d_{b^{\prime}}\left(I^{N}\right)=\emptyset \text { se } b \neq b^{\prime} .
\end{array}
\end{array}\right\}
$$

i.e. o subespaço de $\left[\sqcup_{b \in \underline{n}} I^{N}, I^{N}\right]$ de aplicações definidas em cada cubo por translações e dilatações positivas em cada coordenada com interiores disjuntos. Os grupos $\mathbb{S}_{\underline{n}}$ agem em $C^{N}(\underline{n})$ trocando os índices dos $d_{b}$, e os morfismos estruturais $\mu^{C^{N}}$ compões os pequenos cubos segundo a seguinte fórmula:

$$
\mu_{\underline{\underline{n},\left(\underline{n^{j}}\right){ }_{j \in \underline{n}}}}^{C^{N}}\left(d_{\underline{n}},\left(d_{\underline{n^{j}}}^{j}\right)_{j \in \underline{n}}\right)_{b}:=d_{j} d_{b-\sum_{l=1}^{j} n^{l}}^{j-1} \text { se } \sum_{l=1}^{j-1} n^{l}<b \leq \sum_{l=1}^{j} n^{l}
$$

As degenerações deletam os pequenos $N$-cubos indexados pelos elementos que não estão contidos na imagem da inclusão.

Temos inclusões naturais de operads $-\times 1_{I} \in O p(\mathrm{Top})\left(C^{N}, C^{N+1}\right)$ e definimos $C^{\infty}:=\operatorname{colim} C^{N}$. Note que para todo $n \in \mathbb{N}$ o espaço $C^{\infty}(\underline{n})$ é contrátil.

Teorema 4.1.7. As imagens de $\Omega^{N}$ são naturalmente $C^{N}$-álgebras.

Demonstração: Primeiro assuma $N<\infty$. Seja $\theta^{N}: C^{N} \Omega^{N} \Rightarrow \Omega^{N}$ a transformação natural definida por

$$
\begin{aligned}
\theta_{X}^{N} \in \mathrm{Top}_{*}\left(C^{N} \Omega^{N} X, \Omega^{N} X\right) \\
{\left[d_{\underline{n}}, \gamma^{\underline{n}}\right] \mapsto\left(t \mapsto\left\{\begin{array}{ll}
\gamma^{b}\left(d_{b}^{-1}(t)\right), & t \in d_{b}\left(I^{N}\right) \\
X_{*}, & t \notin d_{\underline{n}}\left(\amalg_{\underline{n}} I^{N}\right)
\end{array}\right)\right.}
\end{aligned}
$$

É fácil verificar que $\theta^{N}$ é compatível com os morfismos estruturais de operad de $C^{N}$.

A ação de $C^{\infty}$ nas imagens de $\Omega^{\infty}$ é induzido dos casos finitos. Por definição um elemento $d_{\underline{n}} \in C^{\infty}(\underline{n})$ pertence a algum $C^{N}(\underline{n}) \operatorname{com} N<\infty$. Podemos então usar a construção acima para definir uma transformação natural $\theta^{\infty}$ compatível com os morfismos estruturais de 2-operad de 
$C^{\infty}$

Seja $N \in \mathbb{N}$. O operad de Fulton-McPherson $\mathcal{F} \mathcal{M}^{N}$ é dado por compactificações de espaços de configurações de pontos em $\mathbb{R}^{N}$. Uma descrição detalhada pode ser encontrada em [LV14]. Temos para cada $n \in \mathbb{N}$ o funtor

$$
\begin{aligned}
\operatorname{Conf}_{n}: \text { Top } & \rightarrow \text { Top } \\
X & \mapsto\left\{x \in[\underline{n}, X] \mid x(b) \neq x\left(b^{\prime}\right) \text { se } b \neq b^{\prime}\right\} .
\end{aligned}
$$

Temos que os espaços $\operatorname{Conf}_{n}\left(\mathbb{R}^{N}\right)$ admitem uma ação de $\mathbb{R}^{N} \rtimes \mathbb{R}_{0}^{+}$por translação e dilatação positiva. Definimos então o $\mathbb{S}$-espaço $F M^{N}(\underline{n}):=\operatorname{Conf}_{n}\left(\mathbb{R}^{N}\right) /_{\mathbb{R}^{N} \rtimes \mathbb{R}_{0}^{+}}$. Temos que $F M^{N}(\underline{n})$ é homeomorfo à $\left\{x \in \operatorname{Conf}_{n}\left(\mathbb{R}^{N}\right) \mid \sum_{b \in \underline{n}} x(b)=0\right.$ e $\left.\sum_{b \in \underline{n}}\|x(b)\|=1\right\}$ para $n>1$, e é $*$ se $n=0,1$.

$\operatorname{Dados}\left(b, b^{\prime}\right) \in \operatorname{Conf}_{2}(\underline{n})$ defina $\theta_{b, b^{\prime}} \in \operatorname{Top}\left(F M^{N}(\underline{n}), \mathbb{S}^{N-1}\right)$ por $\theta_{b, b^{\prime}}(x)=\frac{x\left(b^{\prime}\right)-x(b)}{\left\|x\left(b^{\prime}\right)-x(b)\right\|}$ e dados $\left(b, b^{\prime}, b^{\prime \prime}\right) \in \operatorname{Conf}_{3}(\underline{n})$ defina $\delta_{b, b^{\prime}, b^{\prime \prime}} \in \operatorname{Top}\left(F M^{N}(\underline{n}),[0, \infty]\right)$ por $\delta_{b, b^{\prime}, b^{\prime \prime}}(x)=\frac{\left\|x(b)-x\left(b^{\prime}\right)\right\|}{\left\|x(b)-x\left(b^{\prime \prime}\right)\right\|}$. Considere a aplicação $\rho:=\left(\theta_{\operatorname{Conf}_{2}(\underline{n})}, \delta_{\operatorname{Conf} f_{3}(\underline{n})}\right) \in \operatorname{Top}\left(F M^{N}(\underline{n}),\left(\mathbb{S}^{N-1}\right)^{\operatorname{Conf_{2}}(\underline{n})} \times[0, \infty]^{\operatorname{Conf} f_{3}(\underline{n})}\right)$ que é um homeomorfismo em sua imagem. O operad de Fulton-MacPherson de dimensão $N$ é dado pelo $\mathbb{S}$-espaço $\mathcal{F} \mathcal{M}^{N} \operatorname{com} \mathcal{F} \mathcal{M}^{N}(\underline{n}):=\overline{\rho\left(F M^{N}(\underline{n})\right)}$, i.e. $\mathcal{F} \mathcal{M}^{N}(\underline{n})$ é o fecho da imagem de $\rho$ em $\left(\mathbb{S}^{N-1}\right)^{\operatorname{Conf}_{2}(\underline{n})} \times[0, \infty]^{\operatorname{Conf}_{3}(\underline{n})}$.

Intuitivamente um elemento $x \in \mathcal{F} \mathcal{M}^{N}(\underline{n})$ pode ser considerado uma "configuração virtual"em que pontos podem estar infinitesimalmente próximos de forma que a direção relativa entre dois pontos e as distancias relativas entre três pontos sempre está bem definida e são dadas pelas extensões das funções $\theta$ e $\delta$ para todo o $\mathcal{F} \mathcal{M}^{N}(\underline{n})$. Um ponto $x \in \mathcal{F} \mathcal{M}^{N}(\underline{n})$ está completamente definido pela imagem por essas funções. Temos então que o morfismo estrutural $\mu^{\mathcal{F} \mathcal{M}^{N}}$ é definido por:

$$
\theta_{b, b^{\prime}}\left(\mu_{\left.\underline{n}, \underline{\mathcal{F}} \underline{(n)}^{j}\right)_{j \in \underline{n}}}\left(x,\left(x^{j}\right)_{j \in \underline{n}}\right)\right):= \begin{cases}\theta_{b-\sum_{l=1}^{j-1} n^{l}, b^{\prime}-\sum_{l=1}^{j-1} n^{l}}\left(x^{j}\right), & \text { se } \sum_{l=1}^{j-1} n^{l}<b, b^{\prime} \leq \sum_{l=1}^{j} n^{l} ; \\
\theta_{j, j^{\prime}}(x) & \text { se }\left\{\begin{array}{l}
\sum_{l=1}^{j-1} n^{l}<b \leq \sum_{l=1}^{j} n^{l}, \\
\sum_{l^{\prime}=1}^{j^{\prime}-1} n^{l^{\prime}}<b^{\prime} \leq \sum_{l^{\prime}=1}^{j^{\prime}} n^{l^{\prime}} \\
\left(j, j^{\prime}\right) \in \operatorname{Conf}_{2}(\underline{n})
\end{array}\right.\end{cases}
$$




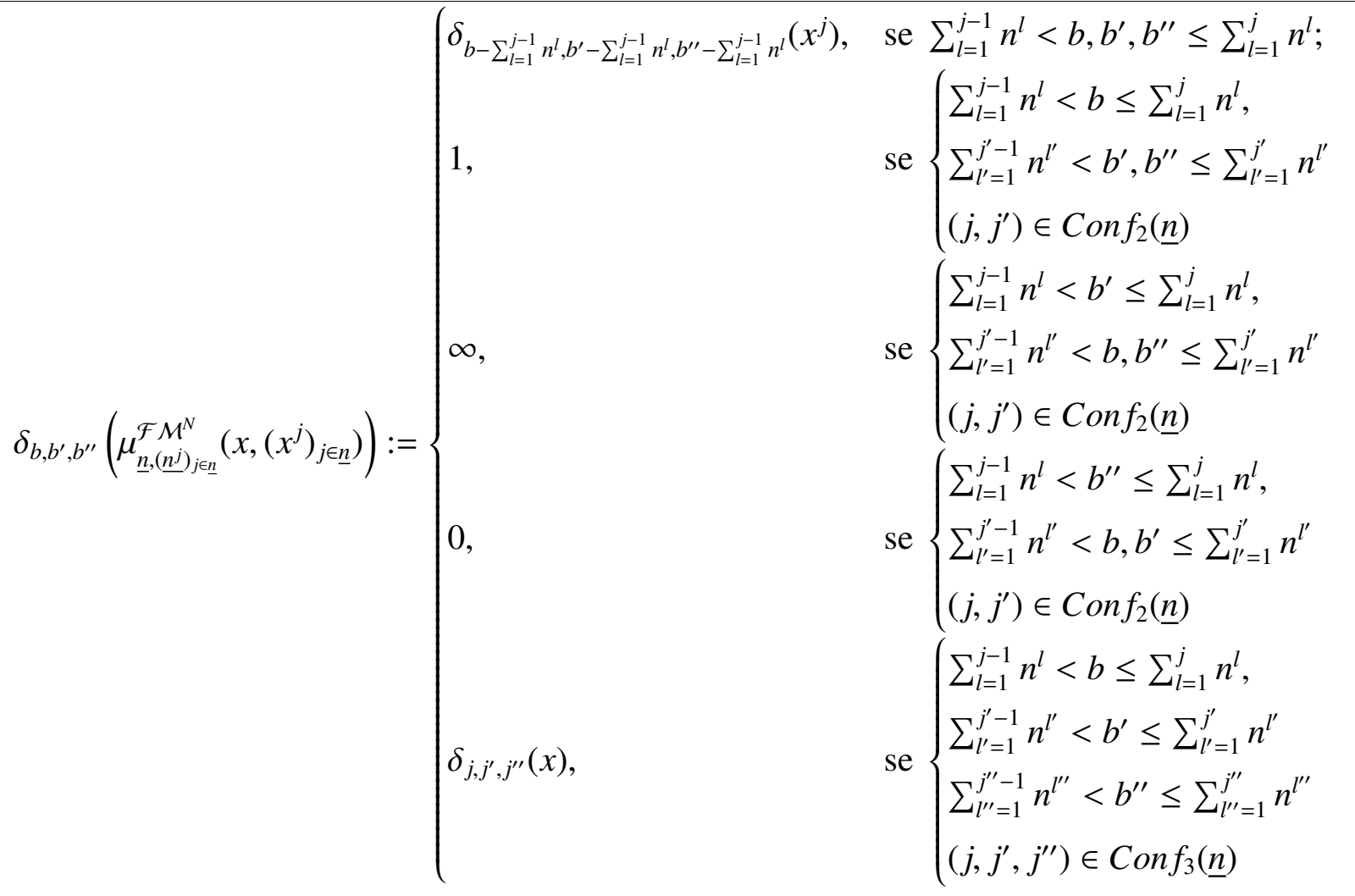

Seguindo a ideia intuitiva acima, essa operação insere $x^{i}$ na configuração de pontos $x$ como uma configuração de pontos infinitesimalmente próximos concentrada no ponto $x(i)$. As degenerações deletam as coordenadas em que o índice aparece.

As inclusões $\iota^{N} \in \operatorname{Top}\left(\mathbb{R}^{N}, \mathbb{R}^{N+1}\right)$ induzem inclusões $\iota^{N} \in 2 O p(\mathrm{Top})\left(\mathcal{F} \mathcal{M}^{N}, \mathcal{F} \mathcal{M}^{N+1}\right)$, e defi$\operatorname{nimos} \mathcal{F} \mathcal{M}^{\infty}:=\operatorname{colim} \mathcal{F} \mathcal{M}^{N}$.

Os espaços $\mathcal{F} \mathcal{M}^{N}(\underline{n})$ são conjuntos semi-algébricos compactos, i.e. um subconjunto compacto de um espaço euclidiano definido por uma sequência finita de equações e inequações polinomiais, o que implica que eles admitem uma triangulação [BCR87], e portanto esses espaços são cofibrantes. Além disso a triangulação pode ser escolhida de forma que as $\mathbb{S}_{\underline{n}}$-ações preservam essa triangulação. Portanto $\mathcal{F} \mathcal{M}^{N}(\underline{n})$ é cofibrante como um $\mathbb{S}$-objeto.

O operad de Barratt-Eccles é composto por espaços totais de fibrados principais universais dos grupos de simetria [BE74]. Seja $G$ um grupo topológico. Defina $G^{\langle\bullet\rangle} \in\left(\mathrm{Top}^{G}\right)^{\Delta^{\mathrm{op}}}$ por:

$$
\begin{aligned}
& G^{\langle\bullet\rangle}: \Delta^{\mathrm{op}} \rightarrow \mathrm{Top}
\end{aligned}
$$

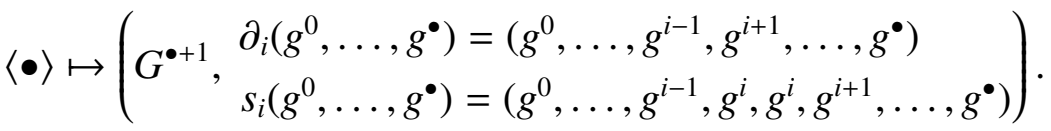

Podemos então definir $E G \in \mathrm{Top}^{G}$ como $E G:=\left|G^{\langle\bullet\rangle}\right|$. Todo ponto de $E G$ é definido por combinações lineares formais $\sum_{i=0}^{\bullet} u^{i} g^{i} \operatorname{com}\left(g^{\langle\bullet\rangle}, u^{\langle\bullet\rangle}\right) \in G^{\bullet+1} \times \Delta^{\langle\bullet\rangle}$. Podemos também definir o $\mathbb{S}$-espaço $E \mathbb{S}$ 
por:

$$
\begin{aligned}
E \mathbb{S}: \mathbb{S}^{\mathrm{op}} & \rightarrow \mathrm{Top} \\
\underline{n} & \mapsto E \mathbb{S}_{\underline{n}}
\end{aligned}
$$

Os morfismos estruturais de operad são dadas por:

$$
\mu_{\left.\underline{n}, \underline{n}^{j}\right)_{j \in \underline{n}}}^{E S}\left(\sum_{l=1}^{q} u^{l} \sigma_{l},\left(\sum_{l^{j}=1}^{q^{j}} u^{l^{j}} \sigma_{l^{j}}^{j}\right)_{j \in \underline{n}}\right):=\sum_{l=1}^{q} \sum_{l^{1}=1}^{q^{1}} \cdots \sum_{l^{n}=1}^{q^{n}} u^{l} u^{l^{1^{1}}} \cdots u^{l^{n}} \mu_{\underline{n}, \underline{(\underline{n} j})_{j \in \underline{\underline{n}}}}^{A s s}\left(\sigma_{l},\left(\sigma_{l^{j}}^{j}\right)_{j \in \underline{n}}\right)
$$

Note que o funtor de componentes conexas $\pi_{0}$ nos dá morfismos $\pi^{C^{1}} \in O p(\mathrm{Top})\left(C^{1}, A s s\right)$, $\pi^{C^{\infty}} \in O p(\mathrm{Top})\left(C^{\infty}, C o m\right), \pi^{\mathcal{F} \mathcal{M}^{\infty}} \in O p(\mathrm{Top})\left(\mathcal{F} \mathcal{M}^{\infty}, C o m\right)$ e $\pi^{E \mathbb{S}} \in O p(\mathrm{Top})(E \mathbb{S}, C o m)$ que são equivalências fracas em $\mathbb{S}-\mathrm{Top}$.

\subsection{2-operads}

A teoria de operads coloridos é uma generalização da teoria de operads onde consideramos operações em múltiplos objetos. Exploramos uma restrição do caso gereal de operads coloridos em duas cores, em que as duas cores são ordenadas. Considere o funtor

$$
\begin{aligned}
-_{*}: \text { Set } & \rightarrow{\mathrm{PO}-\text { Set }_{*}} \\
S & \mapsto S_{*}:=(S \sqcup\{S\}, S)
\end{aligned}
$$

onde a estrutura de ordem parcial é dada por $a>S$ para todo $a \in S$. Considere também o conjunto ordenado $\{c>o\}$.

Definição 4.2.1. Um 2-conjunto é um conjunto $S$ equipado com uma função não-decrescente $\operatorname{cor}_{S} \in \mathrm{PO}-\operatorname{Set}\left(S_{*},\{c>o\}\right)$, a coloração de $S$. A cor de $S$ é $\operatorname{cor}_{S}(S)$. Um 2-conjunto geralmente será denotado simplesmente como $S$ e a coloração $\operatorname{cor}_{S}$ simplesmente como cor quando o 2conjunto $S$ for óbvio do contexto.

Se $\operatorname{cor}(S)=o$ dizemos que $S$ é aberto e se $\operatorname{cor}(S)=c$ dizemos que $S$ é fechado. Denotamos por $S_{c}$ o conjunto de elementos fechados de $S$ e por $S_{o}$ o conjunto de elementos abertos de $S$.

Note que cor ser não-decrescente implica que se $\operatorname{cor}(S)=c$ então $\operatorname{cor}(a)=c, \forall a \in S$.

Definição 4.2.2. Uma 2-função $f$ entre 2-conjuntos $S$ e $S^{\prime}$ é uma função que preserva ponto base $f \in \operatorname{Set}_{*}\left(S_{*}, S_{*}^{\prime}\right)$ tal que $\operatorname{cor}_{S}=\operatorname{cor}_{S^{\prime}} f$.

Note que não há 2-funções entre 2-conjuntos de cores diferentes.

Definição 4.2.3. Seja $2 \mathbb{S}_{\text {inj }}$ a subcategoria da categoria de 2 -conjuntos cujos objetos são os conjuntos fechados $\underline{n}:=\left\{1_{c}, \ldots, n_{c}\right\}$ para todo $n \in \mathbb{N}$ e os conjuntos abertos $\underline{n, m}:=\left\{1_{c}, \ldots, n_{c}, 1_{o}, \ldots, m_{o}\right\}$ 
para todo $(n, m) \in \mathbb{N}^{2}$, e cujos morfismos são as 2-funções injetoras. Seja $2 \mathbb{S}$ a subcategoria de $2 \mathbb{S}_{\text {inj }}$ com os mesmos objetos e cujos morfismos são as 2 -funções bijetoras. Definimos também as subcategorias plenas $2 \mathbb{S}_{c \text {,inj }}$ e $2 \mathbb{S}_{o \text {,inj }}$ de $2 \mathbb{S}_{\text {inj }}$ compostas dos 2 -conjuntos fechados e abertos respectivamente, e analogamente definimos as subcategorias plenas $2 \mathbb{S}_{c}$ e $2 \mathbb{S}_{o}$ de $2 \mathbb{S}$ compostas dos 2-conjuntos fechados e abertos respectivamente.

Como no caso de operads, para todo $\underline{n} \in 2 \mathbb{S}_{c}$ as 2 -bijeções $2 \mathbb{S}_{\underline{n}}:=2 \mathbb{S}(\underline{n}, \underline{n})$ formam um grupo isomorfo a $\mathbb{S}_{\underline{\underline{n}}}$. Para todo $\underline{n, m} \in 2 \mathbb{S}_{0}$ as 2 -bijeções $2 \mathbb{S}_{n, m}:=2 \mathbb{S}(\underline{n, m}, \underline{n, m})$ formam um grupo isomorfo a $\mathbb{S}_{\underline{\underline{n}}} \times \mathbb{S}_{\underline{m}}$.

Definição 4.2.4. Seja $\mathcal{T}$ uma categoria monoidal simétrica bicompleta. Um $2 \mathbb{S}$-objeto em $\mathcal{T}$ é um funtor $Q: 2 \mathbb{S}^{\text {op }} \rightarrow \mathcal{T}$ tal que $Q$ é reduzido, i.e. $Q(\underline{0})=*=Q(\underline{0,0})$. Denotamos a categoria de $2 \mathbb{S}$-objetos em $\mathcal{T}$ por $2 \mathbb{S}-\mathcal{T}$.

Para cada $Q \in 2 \mathbb{S}-\mathcal{T}$ e $S \in 2 \mathbb{S}$ temos uma ação de $2 \mathbb{S}_{S}$ em $Q(S)$. Nos referimos a $2 \mathbb{S}$-objetos em Top como $2 \mathbb{S}$-espaços.

Definição 4.2.5. Um $2 \mathbb{S}$-espaço $Q$ é $2 \mathbb{S}$-livre se para cada $S \in 2 \mathbb{S}$ a ação de $2 \mathbb{S}_{S}$ em $Q(S)$ for livre.

Novamente a linguagem de árvores nos permite definir uma monada em $2 \mathbb{S}-\mathcal{T}$.

Definição 4.2.6. Seja $S \in 2 \mathbb{S}$. Uma 2-árvore orientada sobre $S$ é uma árvore orientada $T$ sobre o conjunto subjacente de $S$ equipada com uma função não-decrescente $\operatorname{cor}_{T} \in \operatorname{PO}-\operatorname{Set}\left(E_{T},\{c>o\}\right)$ tal que $\operatorname{cor}\left(e_{0}\right)=\operatorname{cor}(S)$ e $\forall a \in S$ temos $\operatorname{cor}(\operatorname{out}(a))=\operatorname{cor}(a)$. Uma ordem em uma 2-árvore $T$ é uma ordem na árvore no sentido da sessão anterior tal que se $\operatorname{cor}(\operatorname{out}(v))=c \operatorname{e} \operatorname{cor}\left(\operatorname{out}\left(v^{\prime}\right)\right)=o$ então $v>v^{\prime}$. Em diagramas denotamos arestas fechadas por linhas sinuosas $\sim$.

Denotamos por $\mathbb{T}_{2}(S)$ a categoria cujos objetos são classes de isomorfismos de 2-árvores ordenadas sobre o 2-conjunto $S$ e cujos morfismos são morfismos de árvores que preservam a cor. Temos duas árvores sem vértices internos, que chamamos de 2-árvore unitária fechada e aberta respectivamente.

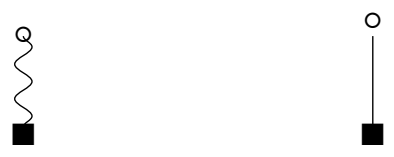

Para uma 2-árvore $T$ e um vértice $v \in V_{T}$ a coloração de $T$ induz uma estrutura de 2-conjunto em in $(v), \operatorname{com} \operatorname{cor}_{i n(v)}(e)=\operatorname{cor}_{T}(e)$ para $e \in \operatorname{in}(v)$ e $\operatorname{cor}_{\mathrm{in}(v)}(\operatorname{in}(v))=\operatorname{cor}_{T}(\operatorname{out}(v))$. Assim como em árvores, temos uma operação de enxerto em 2-árvores.

Como no caso de operads temos que todo $Q \in 2 \mathbb{S}-\mathcal{T}$ e $S \in 2 \mathbb{S}$ induz um funtor

$$
\begin{aligned}
Q: \mathbb{T}_{2}(S)^{\mathrm{op}} & \rightarrow \mathcal{T} \\
T & \mapsto \bigotimes_{v \in V_{T}} Q(\operatorname{in}(v))
\end{aligned}
$$


O somando associado às 2-árvores unitárias é $\mathbb{1}$.

Definição 4.2.7. Para $Q \in 2 \mathbb{S}-\mathcal{T}$ defina o $2 \mathbb{S}$-objeto

$$
\begin{aligned}
\mathbb{T}_{2} Q: 2 \mathbb{S}^{\mathrm{op}} & \rightarrow \mathcal{T} \\
S & \mapsto \int^{\mathbb{T}_{2}(S)} Q(T)
\end{aligned}
$$

Como antes esta construção define um endofuntor $\mathbb{T}_{2}$ em $\mathcal{T}^{2 \mathbb{S}_{\text {op }}}$ que é o funtor subjacente de uma monada.

Definição 4.2.8. Um 2-operad $Q$ em $\mathcal{T}$ é uma $\mathbb{T}_{2}$-álgebra. Denotamos a categoria de 2 -operads em $\mathcal{T}$ por $2 O p(\mathcal{T})$.

A estrutura de 2-operad de $Q$ é completamente definida pelos morfismos

$$
\eta_{c}^{Q} \in \mathcal{T}(\mathbb{1}, Q(\underline{1})), \quad \eta_{o}^{Q} \in \mathcal{T}(\mathbb{1}, Q(\underline{0,1}))
$$

induzidos das 2-árvores unitárias, para cada $n \in \mathbb{N}^{\text {e }}\left(n^{j}\right)_{j \in \underline{n}} \in \mathbb{N}^{\underline{n}}$, um morfismo

$$
\left.\mu_{\underline{n},(\underline{n})_{j \in \underline{n}}}^{Q} \in \mathcal{T}(Q \underline{n}) \otimes\left(\bigotimes_{j \in \underline{n}} Q\left(\underline{n^{j}}\right)\right), Q\left(\sum_{j=1}^{n} n^{j}\right)\right)
$$

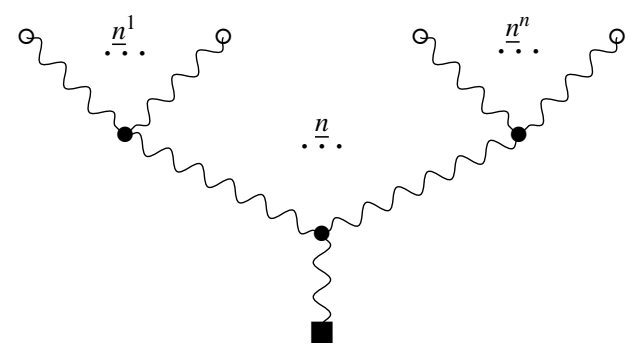

para cada $(n, m) \in \mathbb{N},\left(p^{j}\right)_{j \in \underline{n}} \in \mathbb{N}^{\underline{n}} \mathrm{e}\left(n^{i}, m^{i}\right)_{i \in \underline{m}} \in\left(\mathbb{N}^{2}\right)^{\underline{m}}$, um morfismo

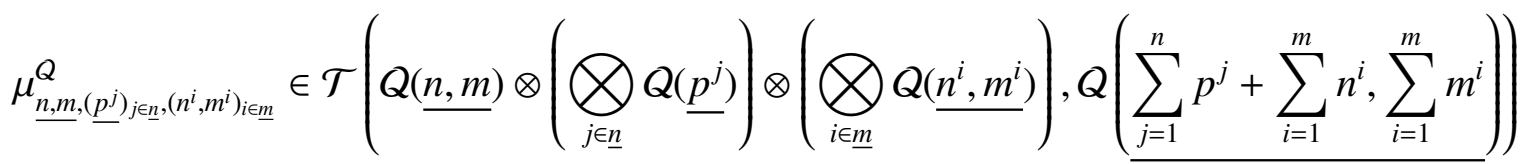

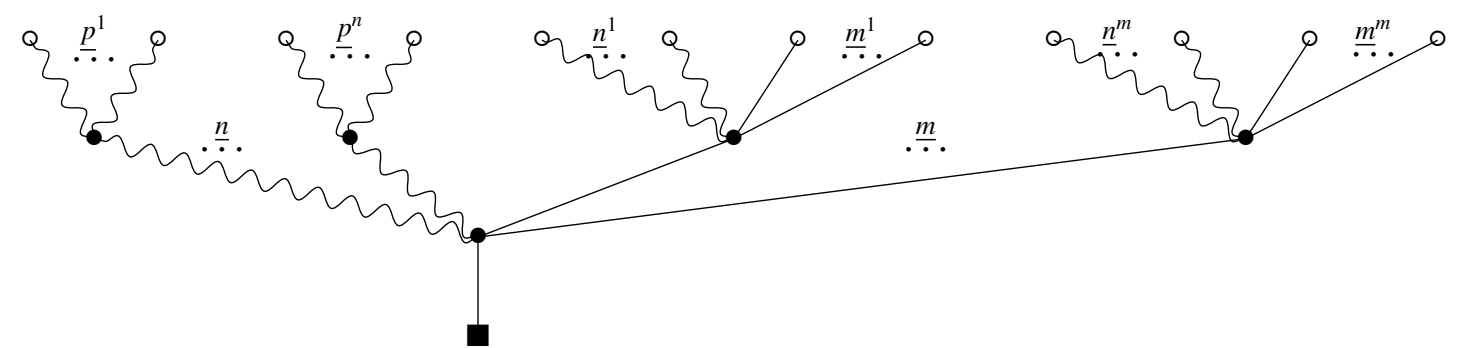

Novamente esses morfismos satisfazem condições de unidade, associatividade e equivariância. 
Todo 2-operad em $\mathcal{T}$ define uma monada em $\mathcal{T}_{*}^{2}$. Se $Q$ é um 2-operad podemos, como na sessão anterior, estender o funtor subjacente de $Q$ em um funtor em $2 \mathbb{S}_{\text {inj }}^{\text {op }}$, e todo $\left(X_{c}, X_{o}\right) \in \mathcal{T}_{*}^{2}$ define um funtor:

$$
\begin{aligned}
\left(X_{c}, X_{o}\right)^{\otimes-}: 2 \mathbb{S}_{\mathrm{inj}} & \rightarrow \mathcal{T} \\
S & \mapsto X_{c}^{\otimes S_{c}} \otimes X_{o}^{\otimes S_{o}}
\end{aligned}
$$

Podemos então definir a monada

$$
\begin{aligned}
Q: \mathcal{T}_{*}^{2} & \rightarrow \mathcal{T}_{*}^{2} \\
\left(X_{c}, X_{o}\right) & \mapsto\left(\int^{2 \mathbb{S}_{c, \mathrm{inj}}} Q(\underline{n}) \otimes X_{c}^{\otimes \underline{n}}, \int^{2 \mathbb{S}_{o, \mathrm{inj}}} Q \underline{(n, m)} \otimes\left(X_{c}, X_{o}\right)^{\otimes n, m}\right)
\end{aligned}
$$

Esse endofuntor admite uma estrutura de monada com a unidade dada por

$$
\left(\eta_{c}^{Q} \otimes 1_{X_{c}}, \eta_{o}^{Q} \otimes 1_{X_{o}}\right) \in \mathcal{T}_{*}\left(\left(X_{c}, X_{o}\right), Q\left(X_{c}, X_{o}\right)\right)
$$

e a multiplicação dada por

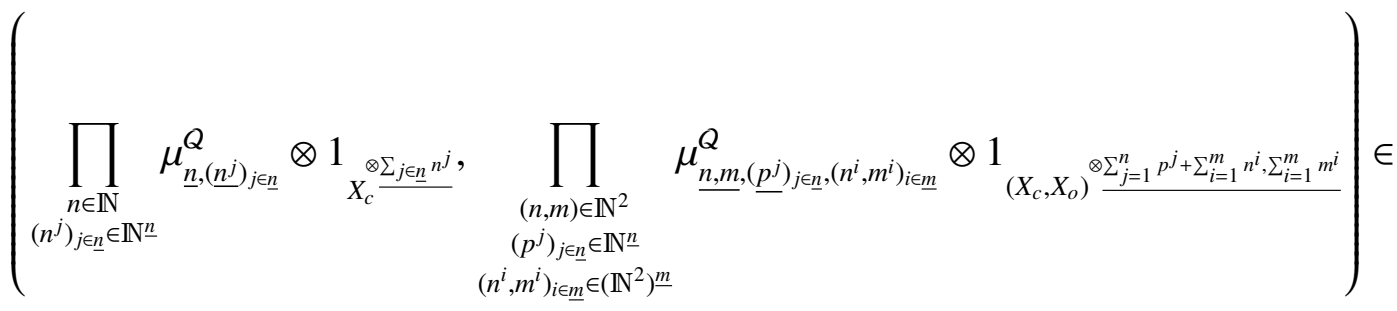

$$
\begin{aligned}
& \mathcal{T}_{*}^{2}\left(Q Q\left(X_{c}, X_{o}\right), Q\left(X_{c}, X_{o}\right)\right) \text {. }
\end{aligned}
$$

Definição 4.2.9. Para um 2-operad $Q$ uma $Q$-álgebra é uma $Q$-álgebra da monada associada $Q$. Denotamos a categoria de $Q$-álgebras por $Q[\mathcal{T}]$.

Para todo 2-operad $Q$ e $\left(X_{c}, X_{o}\right) \in \mathrm{Top}_{*}^{2}$ o espaço $Q\left(X_{c}, X_{o}\right)$ admite uma filtração dupla natural. Seja $F^{l, k} 2 S_{\text {inj }}$ a subcategoria plena de $2 S_{\text {inj }}$ contendo apenas os 2-conjuntos fechados $\underline{n} \operatorname{com} n \leq l$ e os 2-conjuntos abertos $n, m$ com $n \leq l$ e $m \leq k$. Podemos então definir $F^{l, k} Q\left(X_{c}, X_{o}\right)$ como a imagem das inclusões naturais de

$$
\left(\int^{F^{k, l_{2} \mathbb{S}_{c, \mathrm{inj}}}} Q(\underline{n}) \otimes X_{c}^{\otimes n}, \int^{F^{l, k} 2 \mathbb{S}_{o, \mathrm{inj}}} Q(\underline{n, m}) \otimes\left(X_{c}, X_{o}\right)^{\otimes \underline{n, m}}\right)
$$

em $Q\left(X_{c}, X_{o}\right)$. Claramente isso define uma filtração $F^{k} Q\left(X_{c}, X_{o}\right):=\cup_{l \in \mathbb{N}} F^{l, k} Q\left(X_{c}, X_{o}\right)$.

Damos agora alguns exemplos de 2-operads em Top. O operad mais simples é o operad $\mathrm{Com}^{\rightarrow}$ com $\operatorname{Com}^{\rightarrow}(S):=*$ para todo $S \in 2 \mathbb{S}^{\text {op }}$. Os morfismos estruturais são óbvios dado que $*$ é o objeto terminal. As álgebras sobre esse operad são pares de monoides comutativos topológicos $X_{c}, X_{o} \in \operatorname{Com}[\mathrm{Top}]$ equipados com um homomorfismo contínuo $\xi\left(*_{1,0}\right) \in \operatorname{Com}[\mathrm{Top}]\left(X_{c}, X_{o}\right)$ 
induzido pelo elemento de $*_{1,0} \in \operatorname{Com}^{\rightarrow}(\underline{1,0})$.

Também temos o operad $A s s^{\text {act }}$ com $2 \mathbb{S}$-espaço subjacente dado por

$$
\begin{aligned}
\operatorname{Ass}^{\mathrm{act}} \underline{(n, m)}: & = \begin{cases}\mathbb{S}_{\underline{n}} \times \mathbb{S}_{\underline{m}}, & \text { se } m=0,1 \\
\emptyset, & \text { se } m>1 .\end{cases} \\
\operatorname{Ass}^{\mathrm{act}}(\underline{n}) & :=\mathbb{S}_{\underline{\underline{n}}}
\end{aligned}
$$

e com as $\mathbb{S}_{S}$-ações nos espaços não triviais dadas pela ação do grupo de simetrias em si mesmo. Os morfismos estruturais de 2-operads são dados na parte fechada simplesmente por $\mu^{A s s}$ e na parte aberta por

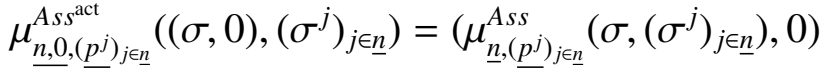

$$
\begin{aligned}
& \mu_{\underline{n, 1},\left(\underline{p^{j}}\right)_{j \in \underline{n}},\left(\underline{n^{\prime}, m^{\prime}}\right)}^{A s s^{\mathrm{act}}}\left((\sigma, 1),\left(\sigma^{j}\right)_{j \in \underline{n}},\left(\sigma^{\prime}, \tau^{\prime}\right)\right)=\left(\mu_{\left.\underline{n}, \underline{\left(p^{j}\right.}\right)_{j \in \underline{\underline{n}}}}^{A s s}\left(\sigma,\left(\sigma^{j}\right)_{j \in \underline{n}}\right) \triangleright \sigma^{\prime}, \tau^{\prime}\right)
\end{aligned}
$$

$\operatorname{com} \tau^{\prime} \in\{0,1\} \mathrm{e}$

$$
\sigma_{\underline{n^{\prime}}}^{\prime} \triangleright \sigma_{\underline{n}}(j)=\left\{\begin{array}{ll}
\sigma_{\underline{n^{\prime}}}^{\prime}(j), & \text { se } 1 \leq j \leq n^{\prime} \\
\sigma_{\underline{n}}\left(j-n^{\prime}\right)+n^{\prime}, & \text { se } n^{\prime}+1 \leq j \leq n^{\prime}+n
\end{array} .\right.
$$

As álgebras sobre esse operad são pares $\left(X_{c}, X_{o}\right) \operatorname{com} X_{c}$ um monoide topológico equipados com uma ação (que não necessariamente preserva ponto base) de $X_{c}$ em $X_{o}$ induzida pelo elemento $\sigma_{1,1} \in A s s^{\text {act }}(\underline{1,1})$,i.e. temos um homomorfismo contínuo $\left[X_{o}, \xi\left(\sigma_{1,1}\right)\right] \eta_{X_{c}}^{X_{o}} \in A s s[\mathrm{Top}]\left(X_{c},\left[X_{o}, X_{o}\right]\right)$, com a estrutura de Ass-álgebra em $\left[X_{o}, X_{o}\right]$ dada por composição.

O operad dos $N$-queijos suíços $S C^{N}$ é definido por $S C^{N}(\underline{n}):=C^{N}(\underline{n})$ e

$\mathcal{S} C^{N}(\underline{n, m}):=\left\{\begin{array}{l|l}d_{n, m}:=\sqcup_{a \in n, m} d_{a} \in\left[\sqcup_{a \in \underline{n}, m} I^{N}, I^{N}\right] & \begin{array}{l}\exists M_{a} \in M_{N \times N}^{\mathrm{dgnl}}\left(\mathbb{R}^{+}\right), C_{a} \in I^{N} \text { t.q. } d_{a}(t)=M_{a} t+C_{a} ; \\ d_{a}\left(I^{N}\right) \cap d_{a^{\prime}}\left(I^{N}\right)=\emptyset \text { se } a \neq a^{\prime} ; \\ C_{a}^{1}=0 \text { se } \operatorname{cor}(a)=o .\end{array}\end{array}\right\}$

i.e. o subespaço de $\left[\sqcup_{a \in S} I^{N}, I^{N}\right]$ de aplicações definidas em cada $N$-cubo por translações e dilatações em cada coordenada com interiores disjuntos tais que as bases na primeira coordenada dos cubos indexados por elementos abertos são mapeados na base na primeira coordenada, com as ações de $\mathbb{S}_{S}$ trocando os índices dos $d_{a}$. A estrutura de operad $\mu^{\mathcal{S C}}$ é dada por composição dos $d_{a}$ de forma análoga à $\mu^{C^{N}}$.

Temos inclusões naturais de 2-operads $-\times 1_{I} \in 2 O p(\mathrm{Top})\left(\mathcal{S C} C^{N}(S), \mathcal{S C} C^{N+1}(S)\right)$ e definimos $\mathcal{S C} C^{\infty}:=\operatorname{colim} S C^{N}$.

Proposição 4.2.10. As imagens de $\Omega_{2}^{N}$ são naturalmente $S C^{N}$-álgebras.

Demonstração: Primeiro assuma $N<\infty$. Seja $\theta_{2}^{N}: S C^{N} \Omega_{2}^{N} \Rightarrow \Omega_{2}^{N}$ a transformação natural 
definida por

$$
\begin{aligned}
& \theta_{c l}^{N}\left(\left[d_{\underline{n}}, \delta^{\underline{n}}\right]\right)(t)= \begin{cases}\delta^{j}\left(d_{j}^{-1}(t)\right), & t \in d_{j}\left(I^{N}\right) \\
*, & t \notin d_{\underline{n}}\left(\coprod_{\underline{n}} I^{N}\right)\end{cases} \\
& \theta_{o \iota}^{N}\left(\left[d_{\underline{m, n}},\left((\alpha, \gamma)^{\underline{m}}, \delta^{\underline{n}}\right)\right]\right)(s)=
\end{aligned}
$$

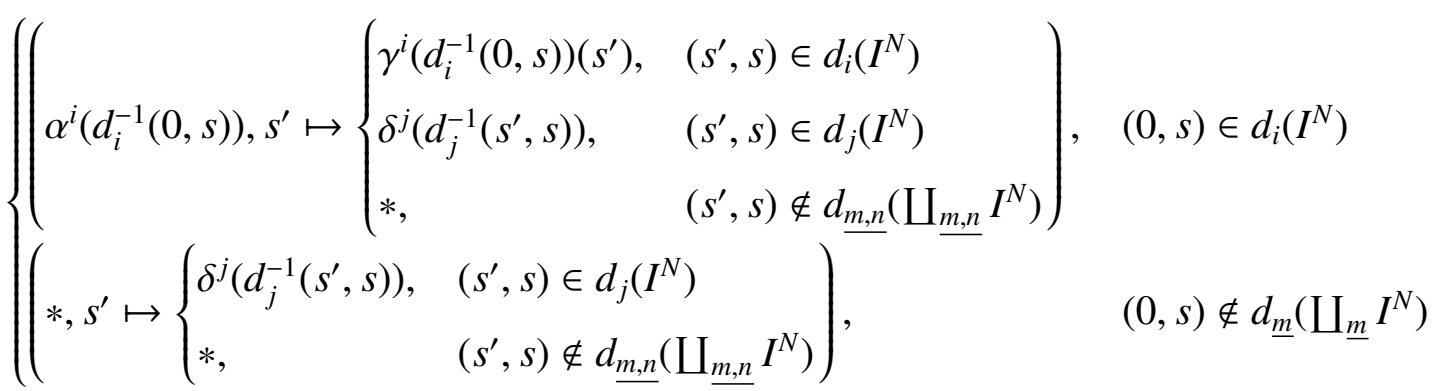

É fácil verificar que $\theta_{2}^{N}$ é compatível com os morfismos estruturais de 2-operad de $\mathcal{S} C^{N}$.

A ação de $S C^{\infty}$ na imagem de $\Omega_{2}^{\infty}$ é induzida dos casos finitos. Por definição um elemento $d_{S} \in \mathcal{S} C^{\infty}(S)$ pertence a algum $\mathcal{S} C^{N}(S) \operatorname{com} N<\infty$. Podemos então usar a construção acima para os casos finitos para definir uma transformação natural $\theta^{\infty}$ compatível com os morfismos estruturais de 2-operad de $S C^{\infty}$

Seja $N \in \mathbb{N}$. O 2-operad de Fulton-McPherson $\mathcal{F} \mathcal{M}_{2}^{N}$ é dado pelo $2 \mathbb{S}$-espaço que nas componentes fechadas é $\mathcal{F} \mathcal{M}_{2}^{N}(\underline{n}):=\mathcal{F} \mathcal{M}^{N}(\underline{n})$ e nas componentes abertas é dado por compactificações de espaços de configurações de pontos coloridos em $\mathbb{H}^{N}=\left\{x^{\underline{N}} \in \mathbb{R}^{N} \mid x^{1} \geq 0\right\}$ com pontos abertos restritos ao bordo. Temos para cada par $n, m \in \mathbb{N}$ o funtor

$$
\begin{aligned}
& \operatorname{Conf}_{n, m}: \operatorname{Top}^{\rightarrow} \rightarrow \operatorname{Top} \\
& \iota \in \operatorname{Top}(A, X) \mapsto \operatorname{Conf}_{n+m}(X) \times_{[\underline{m}, X]} \operatorname{Conf}_{m}(A)
\end{aligned}
$$

Fixe $\iota^{N} \in \operatorname{Top}\left(\partial \mathbb{H}^{N}, \mathbb{H}^{N}\right)$ a inclusão do bordo. Note que $\operatorname{Con} f_{n, m}\left(\iota^{N}\right)$ admitem uma ação de $\mathbb{R}^{N-1} \rtimes \mathbb{R}_{0}^{+}$por translação paralela ao bordo e dilatação positiva. Definimos então $F M_{2}^{N}(\underline{n, m}):=$ $\operatorname{Conf}_{n, m}\left(\iota^{N}\right) /_{\mathbb{R}^{N-1} \rtimes \mathbb{R}_{0}^{+}}$. Temos que $F M_{2}^{N}(\underline{n, m})$ é homeomorfo à $\left\{x \in \operatorname{Conf}_{n, m}\left(\iota^{N}\right) \mid \operatorname{proj}_{\partial \mathbb{H}^{N}} \sum_{a \in n, m} x(a)=\right.$ 0 e $\left.\sum_{a \in n, m}\|x(a)\|=1\right\}$.

$\operatorname{Dados}\left(i, i^{\prime}\right) \in \operatorname{Conf}_{2}(\underline{n, m})$ defina $\theta_{i, i^{\prime}} \in \operatorname{Top}\left(F M_{2}^{N}(\underline{n, m}), \mathbb{S}^{N-1}\right)$ por $\theta_{i, i^{\prime}}(x)=\frac{x\left(i^{\prime}\right)-x(i)}{\left\|x\left(i^{\prime}\right)-x(i)\right\|}$ e dados $\left(i, i^{\prime}, i^{\prime \prime}\right) \in \operatorname{Conf}_{3}(\underline{n})$ defina $\delta_{i, i^{\prime}, i^{\prime \prime \prime}} \in \operatorname{Top}\left(F M_{2}^{N}(\underline{n, m}),[0, \infty]\right)$ por $\delta_{i, i^{\prime}, i^{\prime \prime \prime}}(x)=\frac{\left\|x(i)-x\left(i^{\prime}\right)\right\|}{\left\|x(i)-x\left(i^{\prime \prime}\right)\right\|}$. Considere a aplicação $\rho_{2}:=\left(\theta_{\operatorname{Conf} f_{2}(n, m)}, \delta_{\operatorname{Conf}_{3}(n, m)}\right) \in \operatorname{Top}\left(F M^{N} \underline{(n, m)},\left(\mathbb{S}^{N-1}\right)^{\operatorname{Conf}_{2}(\underline{n, m})} \times[0, \infty]^{\operatorname{Conf} f_{3}(\underline{n, m})}\right)$ que é um homeomorfismo em sua imagem. Definimos então $\mathcal{F} \mathcal{M}^{N}\left(\underline{n, m)}:=\overline{\rho_{2}\left(F M_{2}^{N}(\underline{n, m})\right)}\right.$. Os morfismos estruturais são dados de forma análoga aos de $\mathcal{F} \mathcal{M}^{N}$ apresentada na sessão anterior. Veremos na sessão 4.4 que os espaços $\mathcal{F} \mathcal{M}^{N}(S)$ admitem estruturas de variedades com cantos, e a estrutura de 2-operad é dada pela inclusão de subvariedades no bordo. 
Pelo mesmo argumento da sessão anterior $\mathcal{F} \mathcal{M}_{2}^{N}$ é cofibrante como $2 \mathbb{S}$-espaços.

As inclusões naturais $i_{c}^{N} \in \mathrm{Top}\left(\mathbb{R}^{N}, \mathbb{R}^{N+1}\right)$ e $i_{o}^{N} \in \mathrm{Top}\left(\mathbb{H}^{N}, \mathbb{H}^{N+1}\right)$ induzem inclusões naturais $i_{2}^{N} \in 2 O p(\mathrm{Top})\left(\mathcal{F} \mathcal{M}_{2}^{N}, \mathcal{F} \mathcal{M}_{2}^{N+1}\right)$, e definimos $\mathcal{F} \mathcal{M}_{2}^{\infty}:=\operatorname{colim} \mathcal{F} \mathcal{M}_{2}^{N}$.

O 2-operad de Barratt-Eccles $E \mathbb{S}_{2}$ é definido por $E \mathbb{S}_{2}(\underline{n})=E \mathbb{S}_{\underline{n}}$ e $E \mathbb{S}_{2}(\underline{n, m})=E\left(\mathbb{S}_{\underline{n}} \times \mathbb{S}_{\underline{m}}\right)$ com a estrutura de $2 \mathbb{S}$-espaço dada pelas ações livres. Os morfismos estruturais de 2-operads são os mesmos que $E \mathbb{S}$ na parte fechada e na parte aberta é dado pela seguinte fórmula:

$$
\begin{aligned}
& \left.\mu_{\left.\underline{n, m,}, \underline{p^{j}}\right)_{j \in \underline{n}},\left(\underline{n}^{i}, m^{i}\right)_{i \in \underline{m}}}^{E \mathbb{S}_{2}}\left(\sum_{k=1}^{r} u^{k}\left(\sigma_{k}, \tau_{k}\right),\left(\sum_{l^{j}=1}^{q^{j}} v^{l^{j}} \sigma_{l^{j}}^{j}\right)\right)_{j \in \underline{n}},\left(\sum_{k^{i}}^{r^{i}} u^{k^{i}}\left(\sigma_{k^{i}}^{i}, \tau_{k^{i}}^{i}\right)\right)_{i \in \underline{m}}\right)= \\
& \sum_{k=1}^{r} \sum_{l^{1}=1}^{q^{1}} \cdots \sum_{l^{n}=1}^{q^{n}} \sum_{k^{1}=1}^{r^{1}} \cdots \sum_{k^{m}=1}^{r^{m}} u^{k} v^{l^{1}} \cdots v^{l^{n}} u^{k^{1}} \cdots u^{k^{m}} \\
& \left(\mu_{\underline{n},\left(\underline{p^{j}}\right)_{j \in \underline{\underline{n}}}}^{A s s}\left(\sigma_{k},\left(\sigma_{l^{j}}^{j}\right)_{j \in \underline{n}}\right) \triangleright \mu_{\underline{m},\left(\underline{n^{i}}\right)_{i \in \underline{m}}}^{A s s}\left(\tau_{k},\left(\sigma_{k^{i}}^{i}\right)_{i \in \underline{m}}\right), \mu_{\underline{m},\left(\underline{m^{i}}\right)_{i \in \underline{m}}}^{A s s}\left(\tau_{k},\left(\tau_{k^{i}}^{i}\right)_{i \in \underline{m}}\right)\right)
\end{aligned}
$$

Esse operad também é cofibrante como $2 \mathbb{S}$-espaço.

\subsection{Estrutura modelo em 2-operads e álgebras sobre 2-operads}

Em [BM07] condições para a existência de uma estrutura modelo em operads coloridos e suas álgebras são dadas.

Proposição 4.3.1. Seja $\mathcal{T}$ uma categoria modelo simétrica cofibrantemente gerada equipada com um funtor de substituição fibrante simétrico monoidal Fib e um objeto de intervalo de Hopf I. Então existe uma estrutura modelo cofibrantemente gerada em $2 O p(\mathcal{T})$ transferida da estrutura modelo de $2 \mathbb{S}-\mathcal{T}$ pela adjunção $\left(\mathbb{T}_{2} \dashv U\right)$.

Demonstração: Temos que $2 O p(\mathcal{T})$ é bicompleta, e a demonstração é análoga a demonstração em [GJ95, S.1.5] que a categoria de operads é bicompleta. Para toda monada $C$ o funtor $U$ : $C[\mathcal{T}] \rightarrow \mathcal{T}$ cria limites [BW85, 3.4.1], ou seja os limites de diagramas de 2-operads são dados por limites em $2 \mathbb{S}-\mathcal{T}$, que por sua vez podem ser computados como limites em cada índice. $\mathrm{O}$ funtor $\mathbb{T}_{2}$ reflete colimites filtrados já que o produto tensorial em categorias monoidais fechadas preservam colimites. O 2-operad inicial é o 2-operad de pares pontuados $\overline{\mathbb{1}} \operatorname{com} \overline{\mathbb{1}}(S):=\mathbb{1}$ se $S=\underline{1}, \underline{0,1}, \overline{\mathbb{1}}(S):=*$ se $S=\underline{0}, \underline{0,0}$ e $\overline{\mathbb{1}}(S):=\emptyset$ em todos os outros casos. Uma $\overline{\mathbb{1}}$-álgebra é um par de objetos pontuados sem nenhuma outra estrutura. Coprodutos são construídos da seguinte forma: Uma 2-árvore bicolorida $T$ sobre um 2-conjunto $S$ é uma 2-árvore em $\mathbb{T}_{2}(S)$ com os vértices internos coloridos nas cores preta e branca tais que se para um vértice $v$ existe um vértice $w$ preto (branco) com $\operatorname{pre}(w)=v$ então existe um vértice branco (preto) $w^{\prime}$ tal que pre $\left(w^{\prime}\right)=v$, e denotamos a categoria de 2-árvores bicoloridas sobre $S$ por $\mathbb{T}_{2}^{\mathrm{pb}}(S)$. Para 2-operads $\mathcal{P}$ e $\mathcal{Q}$, uma 
2-árvore bicolorida $T$ e um vértice $v \in V_{T}$ definimos então para todo $S \in 2 \mathbb{S}$

$$
\begin{aligned}
Q \oplus \mathcal{P}: \mathbb{T}_{2}^{\mathrm{pb}}(S)^{\mathrm{op}} & \rightarrow \mathcal{T} \\
T & \mapsto \bigotimes_{v \in V_{T}} \begin{cases}\mathcal{P}(\mathrm{in}(v)) & \text { se } v \text { é preto; } \\
Q(\mathrm{in}(v)) & \text { se } v \text { é branco }\end{cases}
\end{aligned}
$$

e

$$
\begin{aligned}
\mathcal{P} \sqcup Q: 2 \mathbb{S}^{\mathrm{op}} & \rightarrow \mathcal{T} \\
S & \mapsto \int^{\mathrm{T}_{2}^{\mathrm{pb}}(S)} \mathcal{Q} \oplus \mathcal{P}(T) .
\end{aligned}
$$

Podemos verificar que $Q \sqcup \mathcal{P}$ admite uma estrutura de 2-operad e satisfaz a condição universal de coproduto. Para obter o pushout note que para todo 2-operad $O$ temos um endomorfismo $\tau \in$ $2 \mathbb{S}-\mathcal{T}(O \sqcup O, O \sqcup O))$ obtido invertendo as cores dos vértices. Se temos $\phi \in 2 \mathbb{S}-\mathcal{T}(O, \mathcal{P})$ e $\psi \in 2 \mathbb{S}-\mathcal{T}(O, Q)$ então podemos tomar o coequalizador em $\mathbb{S}-\mathcal{T}$ de $\phi \sqcup \psi$ e $(\phi \sqcup \psi) \tau$. Esse $2 \mathbb{S}$-objeto admite uma estrutura de 2-operad e é o pushout $Q \sqcup_{O} \mathcal{P}$.

As condições da proposição 1.4.9 são satisfeitas [BM03, BM07]. O fato que Fib é simétrico monoidal implica que ele induz um funtor de substituição fibrante em $2 O p(\mathcal{T})$ já que temos um isomorfismo natural $\mathbb{T}_{2} \mathrm{Fib} \cong \mathrm{Fib} \mathbb{T}_{2}$. Suponha agora que $Q$ é um operad fibrante. Defina o $2 \mathbb{S}$-objeto $Q^{I}$ como $Q^{I}(S)=\left[\bigotimes_{a \in S} I, Q(S)\right]$, que admite uma estrutura de 2-operad induzida pela estrutura de 2-operad de $Q$ e das estruturas de monoide comutativo e comonoide de $I$, com a comutatividade da estrutura de monoide garantindo a equivariância dos morfismos estruturais. Analogamente temos operads $Q^{\mathbb{1}}$ e $Q^{\mathbb{1} \sqcup \mathbb{1}}$, com $Q^{\mathbb{1}} \cong Q$, e morfismos de operads $\left[\varepsilon, 1_{Q}\right] \in O p(\mathcal{T})\left(Q, Q^{I}\right)$ e $\left[(0,1), 1_{Q}\right] \in$ $O p(\mathcal{T})\left(Q^{I}, Q^{\mathbb{1} \sqcup \mathbb{1}}\right)$. Como $\varepsilon$ admite uma cofibração trivial como sessão pelo axioma da unidade e o lema 1.2.2 então pelo axioma do pushout-produto e da unidade temos que $\bigotimes_{a \in S} 1, \bigotimes_{a \in S} I \in \mathcal{T}_{\text {Cof }}$ e $\bigotimes_{a \in S} \varepsilon \in W_{\mathcal{T}}$, e portanto pela proposição 2.2.4 temos $\left[\varepsilon, 1_{Q}\right] \in W_{2 \mathbb{S}-\mathcal{T}}$. Pelo axioma pushoutproduto e da unidade também temos que $\bigotimes_{a \in S}(0,1) \in C_{\mathcal{T}}$, logo pela proposição 2.2.3 temos $\left[(0,1), 1_{Q}\right] \in F_{2 \mathbb{S}-\mathcal{T}}$. Logo $Q^{I}$ é um objeto de caminhos de $Q$.

A seguinte classe de 2-operads será central no teorema de reconhecimento relativo infinito.

Definição 4.3.2. Um $2 E_{\infty}$-operad é uma resolução cofibrante do 2 -operad terminal $\mathrm{Com}^{\rightarrow}$. Equivalentemente é um 2-operad cofibrante cujos espaços são todos contráteis.

Os operads $\mathcal{F} \mathcal{M}_{2}^{\infty}$ e $E \mathbb{S}_{2}$ são cofibrantes em $2 \mathbb{S}$ - Top e todos os seus espaços são contráteis, porém eles não são cofibrantes, pois as aplicações de degeneração não são compatíveis com as estruturas celulares. Na próxima sessão veremos que existe uma resolução funtorial de 2-operads tal que as imagens desses 2-operads por essa resolução são $2 E_{\infty}$-operads.

Proposição 4.3.3. Seja $\mathcal{T}$ uma categoria que satisfaz as condições da proposição 4.3.1 cujo objeto intervalo de Hopf I é cocomutativo. Então para todo 2-operad $Q$ existe uma estrutura modelo 
cofibrantemente gerada em $Q[\mathcal{T}]$ transferida da estrutura modelo em $\mathcal{T}_{*}^{2}$ pela adjunção $(U \dashv Q)$.

Demonstração: Pelo mesmo argumento que 4.3.1 temos que $Q[\mathcal{T}]$ admite todos os limites e todos os colimites filtrados. O objeto inicial é $(*, *)$, que admite uma estrutura de $Q$-álgebra trivial. Seguindo [GJ95, S. 1.6] a existência de pushouts em $Q[\mathcal{T}]$ segue das seguintes duas construções: Para todo morfismo de 2-operads $\phi \in 2 O p(\mathcal{T})(O, \mathcal{P})$ temos um funtor de imagem direta $\phi_{*}: O[\mathcal{T}] \rightarrow \mathcal{P}[\mathcal{T}]$ adjunto à esquerda do funtor $\phi^{*}: \mathcal{P}[\mathcal{T}] \rightarrow O[\mathcal{T}]$ que para uma $\mathcal{P}_{-}$ álgebra $\left(\left(Y_{c}, Y_{o}\right), \zeta\right)$ nos dá a $O$-álgebra $\left(\left(Y_{c}, Y_{o}\right), \zeta\left(\phi \otimes 1_{\left(Y_{c}, Y_{o}\right)}\right)\right)$. Seja $\left(\left(X_{c}, X_{o}\right), \xi\right) \in O[\mathcal{T}]$. Defina $\phi_{*}\left(X_{c}, X_{o}\right)$ como o equalizador dos morfismos inferiores no diagrama abaixo:

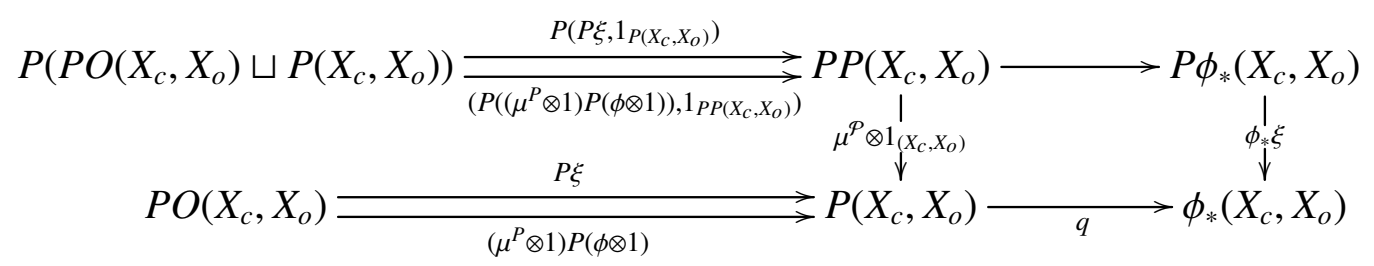

Temos que $P \phi_{*}\left(X_{c}, X_{o}\right)$ é o coequalizador dos morfismos superiores no diagrama e o morfismo estrutural de $\mathcal{P}$-álgebra $\phi_{*} \xi$ de $\phi_{*}\left(X_{c}, X_{o}\right)$ é induzido de $q\left(\mu^{\mathcal{P}} \otimes 1_{\left(X_{c}, X_{o}\right)}\right)$, que coequaliza os morfismos superiores no diagrama. Isso define o funtor $\phi_{*}$.

Além disso para toda $Q$-álgebra $\left(X_{c}, X_{o}\right)$, com morfismo estrutural $\xi \in \mathcal{T}\left(Q\left(X_{c}, X_{o}\right),\left(X_{c}, X_{o}\right)\right)$ existe um 2-operad não reduzido $Q\left[X_{c}, X_{o}\right]$ cuja categoria de álgebras é isomorfa a $Q[\mathcal{T}]^{\left(X_{c}, X_{o}\right) /}$. O $2 \mathbb{S}$-objeto subjacente desse 2-operad é definido por:

$$
\begin{gathered}
Q\left[X_{c}, X_{o}\right](\underline{n}):= \begin{cases}X_{c}, & \text { se } n=0 \\
\int^{2 \mathbb{S}_{c}} Q\left(\underline{n+l)} \otimes X_{c}^{\otimes l},\right. & \text { se } n>0\end{cases} \\
Q\left[X_{c}, X_{o}\right] \underline{(\underline{n, m})}:= \begin{cases}X_{o}, & \text { se }(n, m)=(0,0) \\
\int^{2 \mathbb{S}_{o}} Q \underline{(n+l, m+k)} \otimes\left(X_{c}, X_{o}\right) \underline{\otimes l, k}, & \text { se } n+m>0\end{cases}
\end{gathered}
$$

O morfismo estrutural de 2-operad é induzido de $\mu^{Q}$ e $\xi$. Uma $Q\left[X_{c}, X_{o}\right]$-álgebra $\left(Z_{c}, Z_{o}\right)$ é definida por um morfismo estrutural $\bar{\zeta} \in \mathcal{T}\left(Q\left[X_{c}, X_{o}\right]\left(Z_{c}, Z_{o}\right),\left(Z_{c}, Z_{o}\right)\right)$. Composição com a inclusão natural $i \in \mathcal{T}\left(Q\left(Z_{c}, Z_{o}\right) \sqcup\left(X_{c}, X_{o}\right), Q\left[X_{c}, X_{o}\right]\left(Z_{c}, Z_{o}\right)\right.$ determina dois morfismos $\zeta \in \mathcal{T}\left(Q\left(Z_{c}, Z_{o}\right),\left(Z_{c}, Z_{o}\right)\right)$ e $z \in \mathcal{T}\left(\left(X_{c}, X_{o}\right),\left(Z_{c}, Z_{o}\right)\right)$. Duas aplicações dessa forma nos dão uma estrutura de $Q\left[X_{c}, X_{o}\right]$-álgebra em $\left(Z_{c}, Z_{o}\right)$ se e somente se $\left(Z_{c}, Z_{o}\right)$ é uma $Q$-álgebra sob $\left(X_{c}, X_{o}\right)$.

Suponha agora que temos $z \in Q[\mathcal{T}]\left(\left(X_{c}, X_{o}\right),\left(Z_{c}, Z_{o}\right)\right)$ e $f \in Q[\mathcal{T}]\left(\left(X_{c}, X_{o}\right),\left(Y_{c}, Y_{o}\right)\right)$. O pushout $\left(Z_{c}, Z_{o}\right) \sqcup_{\left(X_{c}, X_{o}\right)}\left(Y_{c}, Y_{o}\right)$ é dado então por $Q[f]_{*}\left(Z_{c}, Z_{o}\right)$.

As condições da proposição 1.4 .9 são satisfeitas. Temos que $U$ preserva colimites filtrados. $\mathrm{O}$ funtor de substituição fibrante Fib em $\mathcal{T}$ induz um em $Q[\mathcal{T}]$, e o fato dele ser simétrico monoidal garante que ele é compatível com as estruturas de $Q$-álgebras. Para todo $\left(\left(X_{c}, X_{o}\right), \xi\right) \in Q[\mathcal{T}]$ temos que $\left(\left[I \sqcup *, X_{c}\right]_{*},\left[I \sqcup *, X_{o}\right]_{*}\right) \in Q[\mathcal{T}]$. O morfismo estrutural de $Q[\mathcal{T}]$-álgebra é induzido de $\xi$ e da estrutura comonoidal de $I$,com a cocomutatividade garantindo a equivariância. Pelo mesmo 
argumento que na proposição 4.3.1 isso nos da um objeto de caminhos em $Q[\mathcal{T}]$.

A seguinte proposição nos permite derivar certos funtores construídos com a construção bar.

Proposição 4.3.4. Sejam $\mathcal{T}$ uma categoria que satisfaz as condições do teorema 4.3.3, $Q$ um 2-operad em $\mathcal{T}$ cujo $2 \mathbb{S}$-objeto subjacente é cofibrante e cujas unidades são cofibrações em $\mathcal{T}$, $\mathcal{A}$ uma categoria modelo e $S$ um $Q$-funtor em $\mathcal{A}$ que preserva colimites, objetos cofibrantes, cofibrações entre objetos cofibrantes e equivalências fracas entre objetos cofibrantes. Então

$$
B^{\langle\bullet\rangle}(S, Q,-): Q[\mathcal{T}] \rightarrow \mathcal{A}^{\Delta^{o p}}
$$

preserva objetos cofibrantes e equivalências fracas entre objetos cofibrantes.

Demonstração: $\operatorname{Se}\left(\iota_{c}, \iota_{o}\right) \in C_{\mathcal{T}_{*}^{2}}\left(\left(A_{c}, A_{o}\right),\left(X_{c}, X_{o}\right)\right) \operatorname{com}\left(A_{c}, A_{o}\right) \in \mathcal{T}_{* \text { Cof }}^{2}$ então $\left(\iota_{c}, \iota_{o}\right)^{\otimes-} \in$ $C_{\mathcal{T}^{2 S_{\text {inj }}}}\left(\left(A_{c}, A_{o}\right)^{\otimes-},\left(X_{c}, X_{o}\right)^{\otimes-}\right)$, e pelas hipóteses em $Q$ temos que $\eta^{Q} \in C_{\mathcal{T}^{2 S_{\mathrm{inj}}^{\mathrm{po}}}}(\overline{\mathbb{1}}, Q)$. Portanto a proposição 2.2.8 aplicada em cada coordenada nos dá que

$$
\left(Q\left(\iota_{c}, \iota_{o}\right), \eta_{\left(X_{c}, X_{o}\right)}^{Q}\right) \in C_{\mathcal{T}_{*}}\left(Q\left(A_{c}, A_{o}\right) \sqcup_{\left(A_{c}, A_{o}\right)}\left(X_{c}, X_{o}\right), Q\left(X_{c}, X_{o}\right)\right)
$$

Além disso por 1.1.11.iv) e 1.1.11.ii) temos que $Q$ preserva cofibrações. Analogamente $Q$ preserva cofibrações triviais. Pelo lema de Ken Brown 1.3.6 temos então que $Q$ preserva equivalências fracas entre objetos cofibrantes.

Provamos indutivamente agora que $B^{\langle\bullet\rangle}\left(\mathbb{1}_{\mathcal{T}_{*}}, Q,-\right)$ preserva objetos cofibrantes e equivalências fracas entre objetos cofibrantes. As hipóteses sobre $S$ implicam que o mesmo vale para $B^{\langle\bullet\rangle}(S, Q,-)$. Seja $\left(X_{c}, X_{o}\right) \in Q[\mathcal{T}]_{\text {Cof. }}$ Como $Q$ preserva cofibrações toda álgebra cofibrante em $Q[\mathcal{T}]$ é cofibrante em $\mathcal{T}_{*}$, e portanto

$$
l^{\langle 0\rangle} \in C_{\mathcal{T}_{*}}\left(L B^{\langle 0\rangle}\left(\mathbb{1}_{\mathcal{T}_{*}}, Q,\left(X_{c}, X_{o}\right)\right)=\emptyset,\left(X_{c}, X_{o}\right)\right)
$$

Agora note que para todo $q \in \mathbb{N}$ temos que o quadrado no diagrama abaixo é um pushout

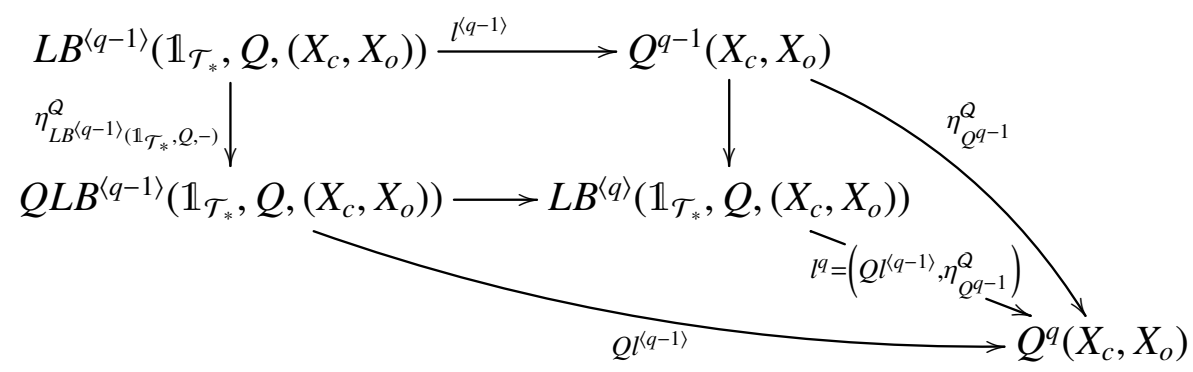

e portanto pelo começo dessa demonstração e por indução $B^{\langle\bullet\rangle}\left(\mathbb{1}_{\mathcal{T}_{*}}, Q,\left(X_{c}, X_{o}\right)\right) \in \mathcal{T}_{* \text { Cof }}^{\Delta^{\text {op }}}$. Como $Q$ preserva equivalências fracas entre objetos cofibrantes também temos que $B^{\langle\bullet\rangle}\left(\mathbb{1}_{\mathcal{T}_{*}}, Q,-\right)$ preserva equivalências fracas entre objetos cofibrantes.

Como a realização geométrica é um adjunto de Quillen à esquerda se a categoria $\mathcal{A}$ na proposição 
acima for uma das categorias topológicas do capítulo 3 então $B(S, Q,-): Q[\mathcal{T}] \rightarrow \mathcal{A}$ admite um funtor derivado à esquerda.

A proposição 2.2.8 e o lema de Ken Brown 1.3.6 implicam que se $\psi \in W_{O p(\mathcal{T})}\left(Q, Q^{\prime}\right)$ é uma equivalência fraca entre 2-operads cofibrantes e $\left(X_{c}, X_{o}\right) \in \mathcal{T}_{* \operatorname{Cof}}$ então $\int^{2 S_{\text {inj }}} \psi(S) \otimes 1_{\left(X_{o}, X_{c}\right)}^{\otimes S} \in$ $W_{\mathcal{T}_{*}^{2}}\left(Q\left(X_{o}, X_{c}\right), Q^{\prime}\left(X_{o}, X_{c}\right)\right)$. Em Top podemos enfraquecer essas hipóteses.

Proposição 4.3.5. Sejam $\psi \in W_{O p(T o p)}\left(Q, Q^{\prime}\right)$ com $Q$ e $Q^{\prime}$ 2-operads $2 \mathbb{S}$-livres e $\left(X_{c}, X_{o}\right) \in T_{o} p_{*}^{2}$ um par de espaços bem pontuados. Então $\int^{2 \mathbb{S}_{i n j}} \psi(S) \times 1_{\left(X_{c}, X_{o}\right)}^{\times S} \in W_{T_{o p}^{2}}\left(Q\left(X_{c}, X_{o}\right), Q^{\prime}\left(X_{c}, X_{o}\right)\right)$.

Demonstração: Segue de um argumento análogo ao da proposição [Ma72, Prop. 3.4]. Como $Q\left(X_{c}, X_{o}\right)$ e $Q^{\prime}\left(X_{c}, X_{o}\right)$ são $\mathcal{H}$-espaços (veja sessão 5.1 para a definição) pelo teorema dual de Whitehead basta provar que $\psi$ induz isomorfismo na homologia com coeficientes em $\mathbb{Z}$. A hipótese do par ser bem pontuado implica que, usando a filtração da sessão $4.2,\left(F^{l, k} Q\left(X_{c}, X_{o}\right), F^{l-1, k} Q\left(X_{c}, X_{o}\right)\right.$ e $\left(F^{l, k} Q\left(X_{c}, X_{o}\right), F^{l, k-1} Q\left(X_{c}, X_{o}\right)\right.$ são pares-RDV com

$$
\begin{gathered}
\left.F^{l, k} Q\left(X_{c}, X_{o}\right)\right|_{F^{l-1, k}} Q\left(X_{c}, X_{o}\right) \cong\left(\int^{\mathbb{S}_{\underline{l}}}(Q(\underline{l}) \sqcup *) \wedge X_{c}^{\wedge \underline{l}}, \int^{\mathbb{S}_{\underline{l}}}\left(Q(\underline{l, k)} \sqcup *) \wedge\left(X_{c}, X_{o}\right)^{\wedge l, k}\right)\right. \\
F^{l, k} Q\left(X_{c}, X_{o}\right) /_{F^{l, k-1}} Q\left(X_{c}, X_{o}\right) \\
\cong\left(*, \int^{\mathbb{S}_{\underline{k}}}(\underline{Q} \underline{(l, k)} \sqcup *) \wedge\left(X_{c}, X_{o}\right)^{\wedge l, k}\right)
\end{gathered}
$$

e temos afirmações equivalentes para $Q^{\prime}$. Basta então que $\psi$ induza isomorfismos na homologia de cada um desses espaços. Temos que $q \in \operatorname{Top}\left((Q(\underline{l}) \sqcup *) \wedge X_{c}^{\underline{l}}, \int^{\mathbb{S}^{\underline{l}}}(Q(\underline{l}) \sqcup *) \wedge X_{c}^{\underline{l}}\right)$ é uma fibração com fibra $\mathbb{S}_{\underline{l}}$, logo ela define uma sequencia espectral convergindo de $E^{2}=H_{\bullet}\left(\mathbb{S}_{\underline{l}}, H_{\bullet}\left((Q(\underline{l}) \sqcup *) \wedge X_{c}^{\underline{l}}\right)\right)$

em $H_{\bullet}\left(\int^{\mathbb{S}_{l}}(Q(\underline{l}) \sqcup *) \wedge X_{c}^{\underline{l}}\right)$. Sendo $Q$ e $Q^{\prime}$ 2S S-livres e $\psi$ uma equivalência fraca então $\psi$ induz um isomorfismo nas paginas $E^{2}$, logo induz um isomorfismo na homologia de $\int^{\mathbb{S}_{l}}(Q(\underline{l}) \sqcup *) \wedge X_{c}^{\underline{l}}$. O mesmo argumento funciona para os outros espaços.

\subsection{Resoluções de Boardman-Vogt}

A pesar dos 2-operads $\mathcal{F} \mathcal{M}_{2}^{N}$ e $E \mathbb{S}_{2}$ da sessão 4.2 serem cofibrantes em $2 \mathbb{S}$ - Top eles não são cofibrantes em $2 O p$ (Top). O problema vem do fato que os morfismos de degeneração nesses operads não preservam a estrutura celular. Seguindo [BV73, BM06] sob as condições do teorema 4.3.1 existe um endofuntor $\mathfrak{B}_{I}: 2 O p(\mathcal{T}) \rightarrow 2 O p(\mathcal{T})$, a resolução de Boardman-Vogt, que transforma 2 -operads que são $2 \mathbb{S}-\mathcal{T}$-cofibrantes e com unidade cofibrante em 2-operads cofibrantes em $2 O p(\mathcal{T})$. A idéia intuitiva da construção $\mathfrak{B}_{I} Q$ para um 2-operad $Q$ é a seguinte (assumindo que os objetos de $\mathcal{T}$ possuem "elementos", a definição formal não faz tal pressuposto): Um elemento de $\mathbb{T}_{2} Q$ é representado por uma 2-árvore $T$ à qual associamos a cada $v \in V_{T}$ um elemento de $Q($ in $(v))$. Um elemento de $\mathfrak{M}_{I} Q$ é um elemento de $\mathbb{T}_{2} Q$ à qual associamos para cada aresta interna um elemento do intervalo de Hopf $I$, que pensamos como o comprimento dessa aresta. Então fazemos dois tipos de identificações. Se a aresta de uma árvore tem comprimento 0 ela é contraída e usamos a estrutura de operad de $Q$ para definir o elemento associado à identificação dos vértices 
nas extremidades. Se um vértice é unitário, no sentido que apenas uma aresta termina nele e ela tem a mesma cor que a saida, e o elemento de $Q(\underline{1})$ associado à esse vértice é uma unidade de $Q$, então removemos esse vértice e identificamos as arestas entrando e saindo dele e associamos à essa nova aresta o produto em $I$ dos comprimentos delas se ambas forem internas, e se uma não for interna simplesmente deletamos o comprimento da outra.

Seja $Q$ um 2-operad. Construímos $\mathfrak{W}_{I} Q$ como um colimite sequencial de morfismos $\mathfrak{w}^{q} \in$ $2 \mathbb{S}-\mathcal{T}\left(\mathfrak{W}_{I}^{q} Q, \mathfrak{W}_{I}^{q+1} Q\right)$, aonde $\mathfrak{W}_{I}^{q} Q$ é a parte de $\mathfrak{W}_{I} Q$ construída com 2-árvores em $T \operatorname{com}\left|E_{T}^{\text {int }}\right| \leq q$. Defina para todo $S \in 2 \mathbb{S}$ e $q \in \mathbb{N}$ a subcategoria plena $\mathbb{T}_{2}(S, q)$ de $\mathbb{T}_{2}(S)$ composta das 2-árvores $T$ tais que $\left|E_{T}^{\text {int }}\right| \leq q$ aonde $E_{T}^{\text {int }}:=s^{-1}\left(V_{T}\right) \backslash e_{0}$. A ordem de $T$ induz uma ordem em $E_{T}^{\text {int }} \operatorname{logo}$ podemos definir

$$
\begin{aligned}
I: \mathbb{T}_{2}(S)^{\mathrm{op}} & \rightarrow \mathcal{T} \\
T & \mapsto \bigotimes_{e \in E_{T}^{\mathrm{int}}} I
\end{aligned}
$$

Defina $\mathfrak{W}_{I}^{0} Q:=Q$. Suponha então que $q>0$, que $\mathfrak{B}_{I}^{q-1} Q$ está definido e que também está definida uma transformação natural

$$
\mathfrak{a}^{q-1}: \int^{\mathbb{T}_{2}(-, q-1)} I(T) \otimes Q(T) \Rightarrow \mathfrak{W}_{I}^{q-1} Q
$$

Para $q=1$ definimos $\mathfrak{a}^{0}$ como as unidades de $Q$ nas 2-árvores unitárias e como a identidade nas 2-corolas.

Podemos definir o funtor

$$
\begin{aligned}
I^{\dagger}: \mathbb{T}_{2}(S)^{\text {op }} & \rightarrow \mathcal{T} \\
T & \mapsto \operatorname{colim}_{D \subseteq E_{T}^{\text {int }}, D \neq \emptyset} I_{D}(T):=\bigotimes_{e \in E_{T}^{\text {int }}} \begin{cases}\mathbb{1}, & \text { se } e \in D ; \\
I, & \text { se } e \notin D .\end{cases}
\end{aligned}
$$

A cofibração trivial $0 \in \mathcal{T}(\mathbb{1}, I)$ e a identidade $1_{I} \in \mathcal{T}(I, I)$ induzem uma transformação natural $0^{\dagger}: I^{\dagger} \Rightarrow I$ tal que pelo axioma pushout-produto $0_{T}^{\dagger} \in C \cap W$ para todo $T \in \mathbb{T}(S)$.

Seja $D \subseteq E_{T}^{\text {int }}$ e $T \in \mathbb{T}_{2}(S)$. Sendo $T /{ }_{D}$ a árvore obtida contraindo as arestas em $D$, i.e. removendo cada $e \in D$ de $E_{T}$ e identificando se e te, então existe um isomorfismo $\kappa^{D} \in \mathcal{T}\left(I_{D}(T), I\left(T /{ }_{D}\right)\right)$.

Seja $V_{T}^{1}:=\left\{v \in V_{T}|| \operatorname{in}(v) \mid=1\right.$ e $\left.\operatorname{cor}(\operatorname{in}(v))=\operatorname{cor}(\operatorname{out}(v))\right\}$ o conjunto de vértices internos com apenas uma aresta entrando neles. Para cada $C \subset V_{T}^{1}$ temos um morfismo $\left\{\mu_{I}, \varepsilon_{I}\right\}_{C} \in \mathcal{T}\left(I_{T}, I_{T / \text { in(C) }}\right)$ induzido por $\mu_{I}$ quando $\operatorname{in}(v)$, out $(v) \in E_{T}^{\text {int }}$ e por $\varepsilon_{I}$ quando $\operatorname{in}(v) \notin E_{T}^{\text {int }} \operatorname{ou} \operatorname{out}(v) \notin E_{T}^{\text {int }}$, que está bem definido pela associatividade de $\mu_{I}$ e por $\varepsilon_{I}$ ser uma counidade de $\mu_{I}$. 
Defina

$$
\begin{aligned}
Q^{\dagger}: \mathbb{T}_{2}(S)^{\mathrm{op}} & \rightarrow \mathcal{T} \\
T & \mapsto \operatorname{colim}_{C \subseteq V_{T}^{1}, C \neq \emptyset} Q_{C}(T):=\bigotimes_{v \in V_{T}} \begin{cases}\mathbb{1}, & \text { se } c \in C ; \\
Q(T), & \text { se } c \notin C .\end{cases}
\end{aligned}
$$

e note que temos uma transformação natural $\eta^{\dagger}: Q^{\dagger} \Rightarrow Q$ induzida pelas unidade de $Q$. Se $Q$ é cofibrante como um $2 \mathbb{S}$-objeto e as unidades de $Q$ são cofibrações então o axioma pushout-produto nos dá que $\eta_{T}^{\dagger} \in C$ para todo $T \in \mathbb{T}(S)$.

Como o produto tensorial preserva colimites temos uma transformação natural

$$
\mathfrak{b}^{q}:=\operatorname{colim}_{D \subseteq E_{T}^{\text {int }}, D \neq \emptyset} \kappa_{D} \otimes \mu_{D}^{Q}: \int^{\mathbb{T}_{2}(-, q)} I^{\dagger}(T) \otimes Q(T) \Rightarrow \int^{\mathbb{T}_{2}(-, q-1)} I(T) \otimes Q(T)
$$

aonde $\mu_{D}^{Q} \in \mathcal{T}\left(Q(T), Q\left(T /{ }_{D}\right)\right)$ é dada pelo morfismo estrutural de 2-operad $\mu^{Q}$. Também temos uma transformação natural

$$
\mathfrak{c}^{q}:=\operatorname{colim}_{C \subseteq V_{T}^{1}, C \neq \emptyset}\left\{\mu_{I}, \varepsilon_{I}\right\}_{C} \otimes \mu_{C}^{Q}: \int^{\mathbb{T}_{2}(-, q)} I(T) \otimes Q^{\dagger}(T) \Rightarrow \int^{\mathbb{T}_{2}(-, q-1)} I(T) \otimes Q(T) .
$$

Definimos então $\mathfrak{B}_{I}^{q} Q$ como o seguinte pushout em $2 \mathbb{S}-\mathcal{T}$ :

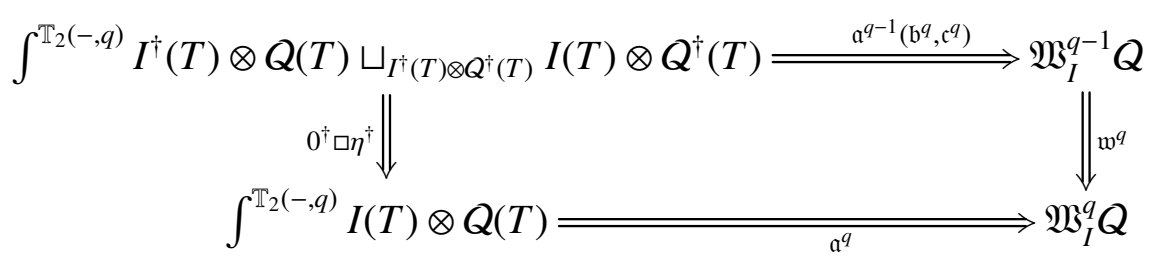

com $\mathfrak{a}^{q}$ definido como o pushout horizontal e $\mathfrak{w}^{q}$ o pushout vertical. Definimos então $\mathfrak{B}_{I} Q:=$ $\operatorname{colim} \mathfrak{B}_{I}^{q} Q$. A estrutura de operad é dada via a operação de enxerto nas 2-árvores. O enxerto de uma 2-árvore em outra cria uma nova aresta interna, e associamo o elemento 1 de $I$ a essa nova aresta. Explicitamente, na componente fechada, dadas $T_{n} \in \mathbb{T}_{2}(\underline{n}), T_{n^{\prime}} \in \mathbb{T}_{2}\left(\underline{n^{\prime}}\right)$ e $a_{c} \in \underline{n^{\prime}}$ temos um morfismo $\mu_{a_{c}}^{I}\left(I\left(T_{n}\right) \otimes \mathbb{1} \otimes I\left(T_{n^{\prime}}\right), I\left(T_{n^{\prime}}\left[T_{n}\right]_{a_{c}}\right)\right)$ induzido de 1. Temos morfismos análogos para os enxertos onde uma ou ambas as árvores são abertas. Esses morfismos juntos com a estrutura de operad livre de $\mathbb{T}_{2} Q$ e os $\mathfrak{a}^{q}$ definem a estrutura de 2-operad de $\mathfrak{B}_{I} Q$.

Em [Sa01] Salvatore provou usando resoluções de Boardman-Vogt que as versões não-reduzida dos operads de Fulton-MacPherson são cofibrantes na categoria de operads não-reduzidos, e a demonstração pode facilmente ser estendida para provar que as versões não-reduzidas dos 2operads de Fulton-MacPherson também são cofibrantes na categoria de 2-operads não reduzidos. A seguinte proposição, provada em [BM06, BM07], nos dá que as resoluções de Boardman-Vogt das versões reduzidas desses 2-operads, assim como o 2-operad de Barratt-Eccles, são cofibrantes 
em $2 O p(\mathcal{T})$.

Proposição 4.4.1. Seja $\mathcal{T}$ uma categoria modelo simétrica cofibrantemente gerada equipada com um funtor de substituição fibrante simétrico monoidal Fib e um objeto de intervalo de Hopf I e $Q$ um 2-operad em $\mathcal{T}$ cujo $2 \mathbb{S}$-objeto subjacente é cofibrante em $2 \mathbb{S}-\mathcal{T}$ e tal que $\eta^{Q}$ é uma cofibração. Então $\mathfrak{B}_{I} Q$ é um 2-operad cofibrante.

Demonstração: Segue do fato que como $\mathcal{T}_{2}$ preserva cofibrações e colimites então $\mathbb{T}_{2} Q$ é cofibrante, e que o morfismo $\iota_{1} \in 2 O p(\mathcal{T})\left(\mathbb{T}_{2} Q, \mathfrak{B}_{I} Q\right)$ obtido atribuindo o elemento 1 à cada aresta interna é uma cofibração. Para detalhes veja [BM06, S. 5].

Em [HoE11] Hoefel provou que os operads e os 2-operads de Fulton-MacPherson não-reduzidos são resoluções cofibrantes dos operads dos pequenos discos e a versão usando discos dos 2-operads dos queijos-suíssos. Provamos agora que o argumento pode ser adaptado para a versão usando cubos usados na presente tese, e que podemos usar as resoluções de Boardman-Vogt para estender esse resultado para as versões reduzidas usadas aqui. Primeiro adaptamos os lemas [HoE11, L. 3.1.1, 3.1.2] para o contexto cúbico. Considere o suboperad $S C_{\square}^{N} \subset S C^{N}$ composto na parte fechada por todos os $d_{\underline{n}} \in C^{N}(\underline{n})$ cujas matrizes $M_{i}$ associados a cada $d_{i}$ para $i \in \underline{n}$ tem todas as entradas da diagonal iguais ao mesmo numero real positivo $m_{i}$, ou seja as imagens $\operatorname{dos} d_{i}$ tem todos os lados do mesmo comprimento $2 m_{i}$, e na parte aberta pelos $d_{\underline{n, m}} \in \mathcal{S} C^{N} \underline{(n, m)}$ cujos $d_{i_{c}}$ para $i_{c} \in \underline{n}$ são dados como na parte fechada e os $d_{i_{o}}$ para $i_{o} \in \underline{m}$ as entradas da matriz $M_{i_{o}}$ são iguais a um $m_{i_{o}}$ a partir da segunda coordenada e a primeira coordenada é $\frac{m_{i_{0}}}{2}$, i.e. as projeções das imagens $\operatorname{dos} d_{i_{o}}$ na primeira coordenada tem os lados do mesmo comprimento $2 m_{i_{o}}$ e o lado da primeira coordenada tem comprimento $m_{i_{o}}$. A inclusão desse suboperad é claramente uma equivalência. Seja $c_{\frac{1}{2}} \in I^{N}$ o centro do $N$-cubo, com todas as coordenadas $\frac{1}{2}$ e $c_{\frac{1}{2}}^{o}$ a projeção de $c_{\frac{1}{2}}$ em $\{0\} \times I^{N-1}$. Defina $\pi \in 2 \mathbb{S}-\operatorname{Top}\left(S C_{\square}^{N}, F M_{2}^{N}\right)$ como

$$
\begin{aligned}
\pi_{\underline{n}}\left(d_{\underline{n}}\right)(i): & =\left[d_{i}\left(c_{\frac{1}{2}}\right)\right] \\
\pi_{\underline{n, m}}\left(d_{\underline{n, m}}\right)(a) & :=\left\{\begin{array}{ll}
{\left[d_{a}\left(c_{\frac{1}{2}}\right)\right],} & \text { se } \operatorname{cor}(a)=c \\
{\left[d_{a}\left(c_{\frac{1}{2}}^{o}\right)\right],} & \text { se } \operatorname{cor}(a)=o
\end{array} .\right.
\end{aligned}
$$

Lema 4.4.2. Para todo $S \in 2 \mathbb{S}$ e $x \in F M_{2}^{N}(S)$ a imagem inversa $\pi^{-1}(x)$ é convexa em $\mathcal{S C}_{\square}^{N}(S)$.

Demonstração: Suponha que temos $d_{S}, d_{S}^{\prime} \in \pi^{-1}(x)$. Precisamos provar que para todo $u \in I$ temos que $u d_{S}+(1-u) d_{S}^{\prime} \in \pi^{-1}(x)$. A única parte não óbvia dessa afirmação é que as imagens dos $u d_{i}+(1-u) d_{i}^{\prime}$ são disjuntas. Tome $\left(i, i^{\prime}\right) \in \operatorname{Conf}_{2}(S)$ e $j_{i, i^{\prime}} \in \underline{N}$ tal que $\theta_{i, i^{\prime}}(x)^{j_{i, i^{\prime}}}=\max _{j \in \underline{N}}\left\{\theta_{i, i^{\prime}}(x)^{j}\right\}$, i.e. $j_{i, i^{\prime}}$ é a coordenada de $\theta_{i, i^{\prime}}(x)$ com maior valor. Então a disjunção dos interiores de $d_{i}$ e $d_{i^{\prime}}$ e dos interiores de $d_{i}^{\prime}$ e $d_{i^{\prime}}^{\prime}$ são dados por $\left|C_{i}^{j_{i, i^{\prime}}}-C_{i^{\prime}}^{j_{i, i^{\prime}}}\right| \geq m_{i}+m_{i^{\prime}}$ e $\left|C_{i}^{j_{i, i^{\prime}}}-C_{i^{\prime}}^{j_{i, i^{\prime}}}\right| \geq m_{i}^{\prime}+m_{i^{\prime}}^{\prime}$. Por uma computação simples podemos ver que

$$
\left|\left(u C_{i}^{j_{i, i^{\prime}}}+(1-u) C_{i}^{\prime j_{i, i^{\prime}}}\right)-\left(u C_{i^{\prime}}^{j_{i, i^{\prime}}}+(1-u) C_{i^{\prime}}^{j_{i, i^{\prime}}}\right)\right| \geq\left(u m_{i}+(1-u) m_{i}^{\prime}\right)+\left(u m_{i^{\prime}}+(1-u) m_{i^{\prime}}^{\prime}\right)
$$


o que nos dá que as imagens dos $u d_{i}+(1-u) d_{i}^{\prime}$ são disjuntas, e assim que $\pi^{-1}(x)$ é convexa.

Para todo $S \in 2 \mathbb{S}$ defina $\widetilde{\mathbb{T}}_{2}(S)$ o subconjunto dos objetos de $\mathbb{T}_{2}(S)$ composto pelas árvores $T$ tais que $E_{T}^{\text {int }} \neq \emptyset$ e para todo $v \in V_{T}$ temos que ou in $(v) \geq 2$ ou que in $(v)=1, \operatorname{cor}(\operatorname{out}(v))=o$ e $\operatorname{cor}(\operatorname{in}(v))=c$. Temos que o bordo de $\mathcal{F} \mathcal{M}_{2}^{N}(S)$ admite uma estratificação indexada por $\widetilde{\mathbb{T}}_{2}(S)$, i.e. existem subespaços disjuntos $\mathcal{F} \mathcal{M}_{2}^{N}(S)_{T} \subset \partial \mathcal{F} \mathcal{M}_{2}^{N}(S)$ para cada $T \in \widetilde{\mathbb{T}}_{2}(S)$ tal que

$$
\mathcal{F} \mathcal{M}_{2}^{N}(S)=\bigcup_{T \in \mathbb{T}_{2}(S)} \mathcal{F} \mathcal{M}_{2}^{N}(S)_{T}
$$

Além disso temos homeomorfismos $\mathcal{F} \mathcal{M}_{2}^{N}(S)_{T} \cong F M_{2}^{N}(T)$. Um sistema de coordenadas para $\mathcal{F} \mathcal{M}_{2}^{N}(S)$ foi construído em [AS94]. Podemos definir para cada $T \in \widetilde{\mathbb{T}}_{2}(S)$ uma aplicação contínua $\phi_{T} \in \operatorname{Top}\left(F M_{2}^{N}(T) \times \mathbb{R}_{\geq 0}^{E_{T}^{\text {int }}},\left[S, \mathbb{R}^{N}\right]\right)$ da seguinte forma: Defina $e_{v}^{a} \in \operatorname{in}(v)$ para todo $a \in S$ e $v<a$ na ordem parcial induzida pelos caminhos como a aresta contida no caminho entre $a$ e $v$. Definimos então $\phi$ como

$$
\phi_{T}\left(\left(x_{v}\right)_{v \in V_{T}}, t^{E_{T}^{\mathrm{int}}}\right):=\left(\sum_{v<a}\left(x_{v}\left(e_{v}^{a}\right) \cdot \prod_{\substack{e \in E_{T}^{\mathrm{int}} \\ e<v}} t^{e}\right)\right)_{a \in S} .
$$

Para todo $x \in \mathcal{F} \mathcal{M}_{2}^{N}(S)_{T}$ existe um aberto $V$ de $F M_{2}^{N}(T)$ e um aberto $W$ de $\mathbb{R}_{\geq 0}^{E_{T}^{\text {int }}}$ tal que $\phi_{T}$, composto com o quociente pela ação por translação e dilatação, define um homeomorfismo entre $V \times W$ e uma vizinhança de $x$. Isso define uma estrutura de variedade com cantos em $\mathcal{F} \mathcal{M}_{2}^{N}(S)$.

Seja $U \subset \mathcal{F} \mathcal{M}_{2}^{N}(S)$ uma vizinhança de colarinho do bordo $\partial \mathcal{F} \mathcal{M}_{2}^{N}(S)$ equipada com um homeomorfismo $h \in \operatorname{Top}\left(\partial \mathcal{F} \mathcal{M}_{2}^{N}(S) \times[0,1), U\right)$ tal que para todo $x \in \partial \mathcal{F} \mathcal{M}_{2}^{N}(S)$ existe uma vizinhança $W \subset \partial \mathcal{F} \mathcal{M}_{2}^{N}(S)$ tal que $h(W \times[0,1))$ está contido em uma vizinhança de $x$ no sistema de coordenadas descrito acima.

Lema 4.4.3. Para todo $S \in 2 \mathbb{S}, x \in \partial \mathcal{F} \mathcal{M}_{2}^{N}(S)$ e $d_{S}, d_{S}^{\prime} \in \pi^{-1}(h(\{x\} \times(0,1)))$ qualquer combinação convexa $u d_{s}+(1-u) d_{S}$ é uma elemento bem definido de $\mathcal{S C}_{\square}^{N}(S)$.

Demonstração: Pela descrição das aplicações $\phi_{T}$ temos que como $d_{S}, d_{S}^{\prime} \in \pi^{-1}(h(\{x\} \times(0,1)))$ então $d_{S}$ pode ser obtido de $d_{S}^{\prime}$ por uma sequência de translações e dilatações. A conclusão então segue do lema 4.4.2.

Teorema 4.4.4. Seja $N \in \mathbb{N} \cup\{\infty\}$. O 2-operad $\mathfrak{W}_{I} \mathcal{F} \mathcal{M}_{2}^{N}$ é uma resolução cofibrante de $\mathcal{S} C^{N}$ em $2 O p(T O P)$.

Demonstração: Suponha primeiro $N<\infty$. Seguindo [HoE11, T. 3.1.3] definimos uma aplicação natural $\bar{v} \in 2 \mathbb{S}-\operatorname{Top}\left(\mathcal{F} \mathcal{M}_{2}^{N}, \mathcal{S} C^{N}\right)$ com a propriedade que ela é compatível com os morfismos de operad exceto as degenerações, definida como uma extensão compatível com a estrutura de 2operad ao bordo de um morfismo $v \in 2 \mathbb{S}-\mathcal{T}\left(F M_{2}^{N}, \mathcal{S} C_{\square}^{N}\right)$ dada da seguinte forma: Para $\underline{0}$ e $\underline{0,0} 0$ só há uma aplicação possível, já que os 2-operads são reduzidos, e definimos $v_{\underline{1}}(*)=1_{I}, v_{1,0}(*)=1_{I}$ 
e $v_{0,1}(*)=1_{I}$. Para todo $n>1$ temos que $F M^{N}(\underline{n})$ é homeomorfo a

$$
\left\{x \in \operatorname{Conf}_{n}\left(\mathbb{R}^{N}\right) \mid \frac{\sum_{i \in \underline{n}} x(i)}{n}=c_{\frac{1}{2}} \text { e } \sum_{i \in \underline{n}}\left\|x(i)-c_{\frac{1}{2}}\right\|=\frac{1}{2}\right\} .
$$

Para todo par $n, m \in \mathbb{N}$ com $n+m>1$ temos que $F M_{2}^{N} \underline{(n, m)}$ é homeomorfo a

$$
\left\{x \in \operatorname{Conf}_{n, m}\left(\mathbb{H}^{N}\right) \mid \operatorname{proj}_{\partial \mathbb{H}^{N}} \frac{\sum_{a \in n, m} x(a)}{n+m}=c_{\frac{1}{2}}^{o} \text { e } \sum_{a \in \underline{n}, m}\left\|x(a)-c_{\frac{1}{2}}^{o}\right\|=\frac{1}{2}\right\} .
$$

Para $|S|>1$ definimos então $v_{S}(x)$ como o elemento de $S C^{N}(S)$ tal que se $a \in S_{c}$ então $v_{S}(x)_{a}\left(c_{\frac{1}{2}}\right)=$ $x(a)$ e se $a \in S_{o}$ então $v_{S}(x)_{a}\left(c_{\frac{1}{2}}^{o}\right)=x(a)$, e tais que os números reais positivos $m_{a}$ que definem as suas matrizes são todos iguais e é o número maximal tais que os interiores dos cubos são disjuntos e contidos em $I^{N}$.

Como $F M^{N}(\underline{2}) \cong \mathbb{S}^{N-1}$ é compacto temos que $F M^{N}(\underline{2})=\mathcal{F} \mathcal{M}^{N}(\underline{2})$ e como $F M_{2}^{N}(\underline{0,2}) \cong \mathbb{S}^{N-2}$ é compacto temos que $F M^{N}(\underline{0,2})=\mathcal{F} \mathcal{M}_{2}^{N}(\underline{0,2})$. Temos portanto que $\bar{v}$ está bem definido nesses casos. suponha que $\bar{v}$ foi bem definido para todo $\underline{l} \operatorname{com} l<n$ de forma que ele é compatível com a estrutura de operad. Como o bordo de $\mathcal{F} \mathcal{M}^{N}(\underline{n})$ é composto de produtos de $\mathcal{F} \mathcal{M}^{N}(\underline{k}) \operatorname{com} l<n$ podemos definir $v^{\prime} \in \mathcal{T}\left(\partial \mathcal{F} \mathcal{M}^{N}(\underline{n}), C^{N}(\underline{n})\right)$ como um morfismo de operads. Dada vizinhança de colarinho $U$ do bordo $\partial \mathcal{F} \mathcal{M}^{N}(\underline{n})$ dada acima e estenda $v^{\prime}$ em $U$ de forma constante ao longo das fibras. Como $\mathcal{F} \mathcal{M}^{N}(\underline{n})$ é compacto existe uma aplicação contínua $u \in \operatorname{Top}\left(\mathcal{F} \mathcal{M}^{N}(\underline{n}), I\right)$ que é constantemente 1 em $\partial \mathcal{F} \mathcal{M}^{N}(\underline{n})$ e é 0 em $\mathcal{F} \mathcal{M}^{N}(\underline{n}) \backslash U$. Definimos $\bar{v}_{\underline{n}}:=(1-u) v_{\underline{n}}+u v_{\underline{n}}^{\prime}$. Como para cada $x \in U$ temos que $v_{\underline{n}}(x), v_{\underline{n}}^{\prime}(x) \in \pi^{-1}(h(\{x\} \times(0,1)))$ o lema 4.4 .3 nos dá que $\bar{v}_{\underline{n}}$ está bem definido. A construção dos $\bar{v}_{\underline{n}, m}$ é análoga.

Temos portanto que como $\bar{v} \in 2 \mathbb{S}-\operatorname{Top}\left(\mathcal{F} \mathcal{M}_{2}^{N}, \mathcal{S} C^{N}\right)$ está definido como um morfismo de operads nos bordos e como uma equivalência homotópica no interior então $\bar{v}_{S}$ são equivalências homotópicas compatíveis com as operações de 2-operads exceto as degenerações. Definimos agora $\mathfrak{v} \in 2 O p(\mathrm{Top})\left(\mathfrak{B}_{I} \mathcal{F} \mathcal{M}_{2}^{N}, \mathcal{S} C^{N}\right)$ como um colimite de morfismos $\mathfrak{v}^{q} \in 2 \mathbb{S}-\operatorname{Top}\left(\mathfrak{M}_{I}^{q} \mathcal{F} \mathcal{M}_{2}^{N}, \mathcal{S} C^{N}\right)$ definidos recursivamente. Definimos $\mathfrak{v}^{0}:=\bar{v}$. Suponha que $\mathfrak{v}^{q-1}$ está bem definido. Seja $S \in 2 \mathbb{S}$ e $T \in \mathbb{T}_{2}(S, q)$. Definimos $\mathfrak{v}_{T} \in \operatorname{Top}\left(I(T) \times \mathcal{F} \mathcal{M}_{2}^{N}(T), \mathcal{S} C^{N}(T)\right)$ como $\mathfrak{v}^{q-1}$ no subespaço $I^{\dagger}(T) \times$ $\mathcal{F} \mathcal{M}_{2}^{N}(T)$ e como $\mu^{S C^{N}} \mathbb{T}_{2} \bar{v}_{T}$ no subespaço $(1, \ldots, 1) \times \mathcal{F} \mathcal{M}_{2}^{N}(T)$. Como as degenerações em $\mathcal{F} \mathcal{M}_{2}^{N}$ preservam as posições relativas dos pontos que não são deletados e $\bar{v}$ é compatível com o resto da estrutura de operad temos pelo lema 4.4.2 que podemos estender $\mathfrak{v}_{T}^{q}$ para todo $I(T) \times \mathcal{F} \mathcal{M}_{2}^{N}(T)$. Essa construção é compatível com as identificações em $\mathfrak{B}_{I}^{q} \mathcal{F} \mathcal{M}_{2}^{N}$ e portanto temos $\mathfrak{v}^{q}$ bem definido. Definimos então $\mathfrak{v}:=$ colim $\mathfrak{v}^{q}$, e por construção ela é uma aplicação de 2-operads. Como por construção $\mathfrak{v}$ é homotópico à composição do morfismo $\mu \in 2 O p(\mathrm{Top})\left(\mathfrak{W}_{I} \mathcal{F} \mathcal{M}_{2}^{N}(S), \mathcal{F} \mathcal{M}_{2}^{N}(S)\right)$ induzido da estrutura de 2-operad com $\bar{v}$ então temos que $\mathfrak{v} \in W_{2 O p(\text { Top })}$. O teorema então segue da proposição 4.4 .1 


\section{Capítulo 5}

\section{Princípio de reconhecimento de espaços de laços rela- tivos}

Nesse capítulo provamos o princípio de reconhecimento de espaços de laços relativos para $2<N \leq \infty$ que afirma o seguinte: Para $2<N<\infty$ o funtor $\Omega_{2}^{N}$ induz uma equivalência entre a categoria homotópica de espaços relativos $N-1$-conexos $\mathcal{H} o \mathrm{Top}_{N-1}$ e a categoria homotópica de $\overline{\mathcal{S} C^{N}}$-álgebras grouplike $\mathcal{H} o \overline{S C}^{N}$ [Top] $]_{\text {Grp }}$ para qualquer resolução cofibrante do 2operad dos $N$-queijos suíços. Em particular podemos usar a resolução de Boardman-Vogt dos 2-operads de Fulton-MacPherson. $\mathrm{O}$ funtor $\Omega_{2}^{\infty}$ induz uma equivalência entre a categoria homotópica de espectros relativos conectivos $\mathcal{H} o \mathrm{Sp}_{0}^{\nearrow}$ e a categoria homotópica de $\mathcal{E}_{2}^{\infty}$-álgebras grouplike $\mathcal{H} o \mathcal{E}_{2}^{\infty}[\mathrm{Top}]_{\text {Grp }}$ para qualquer $2 E_{\infty}$-operad $\mathcal{E}_{2}^{\infty}$. Em particular podemos usar a resolução de Boardman-Vogt do 2-operad de Barratt-Eccles.

Na primeira sessão apresentamos o teorema de aproximação do May [Ma74] e do Cohen [CLM76], que nos diz que temos uma transformação natural de monadas $\alpha^{N}: C^{N} \Rightarrow \Omega^{N} \Sigma^{N}$ que é um completamento de grupo em cada componente se $N>1$. Essa propriedade implica que em $\overline{C^{N}}$-álgebras grouplike essa transformação natural é uma equivalência fraca. Provamos então via a construção de uma quasi-fibração que temos uma transformação natural de monadas $\alpha_{2}^{N}: \mathcal{S} C^{N} \Rightarrow \Omega_{2}^{N} \Sigma_{2}^{N}$ que é um completamento de grupo em cada componente se $N>2$.

Na segunda sessão apresentamos alguns resultados de compatibilidade da realização geométrica com os funtores $\Sigma_{2}^{N}, \Omega_{2}^{N}$ e a monada $Q$ associada a um 2-operad $Q$.

Na terceira sessão mostramos que para $2<N<\infty$ os funtores $\Omega_{2}^{N}$ admitem quasi-adjuntos de Quillen idempotentes à esquerda $B_{2}^{N}$, que podemos usar esses funtores para definir uma quasimonada idempotente de Quillen em $\overline{S C}^{N}$ [Top] cujos objetos fibrantes são as álgebras grouplike e que as equivalências de subcategorias homotópicas associadas são as equivalências do princípio de reconhecimento de espaços de laços relativos.

Na quarta sessão estendemos os resultados da sessão 3 e provamos o principio de reconhecimento para espaços de laços infinitos. Provamos que nesse caso a subcategoria homotópica de espectros relativos conectivos pode ser obtida via uma quasi-comonada idempotente de Quillen. 


\subsection{Teorema de aproximação}

Seja $\alpha^{N}$ a composição das transformações naturais

$$
C^{N} \stackrel{C^{N} \eta^{N}}{\Longrightarrow} C^{N} \Omega^{N} \Sigma^{N} \stackrel{\theta_{\Sigma^{N}}^{N}}{\Longrightarrow} \Omega^{N} \Sigma^{N}
$$

que é explicitamente dada por

$$
\alpha_{X}^{N}\left(\left[d_{\underline{n}}, x^{\underline{n}}\right]\right)(t)=\left\{\begin{array}{ll}
{\left[x^{j}, d_{j}^{-1}(t)\right],} & t \in d_{j}\left(I^{N}\right) \\
*, & t \notin d_{\underline{n}}\left(\amalg_{\underline{n}} I^{N}\right)
\end{array} .\right.
$$

Precisaremos de algumas definições antes de apresentar o teorema de aproximação. Para detalhes veja [Ma72, Ma74, CLM76].

Definição 5.1.1. Um $\mathcal{H}$-espaço é um espaço pontuado $X \in \mathrm{T} o \mathrm{p}_{*}$ equipado com uma aplicação $\mu \in \mathrm{Top}_{*}(X \times X, X)$ tal que $\mu\left(X_{*} \times 1_{X}\right) \simeq 1_{X} \simeq \mu\left(1_{X} \times X_{*}\right)$. Uma $\mathcal{H}$-aplicação entre $\mathcal{H}$-espaços $X$ e $Y$ é uma aplicação $f \in \operatorname{Top}_{*}(X, Y)$ tal que $f \mu^{X} \simeq \mu^{Y}(f \times f)$. Denotamos a categoria de $\mathcal{H}$-espaços por $\mathcal{H}-$ Top.

Para um $\mathcal{H}$-espaço $(X, \mu)$ os grupos de homologia $H_{\bullet}(X ; k)$ para qualquer anel de coeficientes $k$ equipado com o produto de Pontryagin $\mu_{*}$ formam uma $k$-álgebra graduada com unidade $\left[X_{*}\right]$.

Definição 5.1.2. Um $\mathcal{H}$-espaço $X$ é homotopicamente associativo se $\mu\left(1_{X} \times \mu\right) \simeq \mu\left(\mu \times 1_{X}\right)$.

A estrutura de $k$-álgebra graduada de $H_{\bullet}(X ; k)$ para um $\mathcal{H}$-espaço homotopicamente associativo $X$ é associativa.

Para todo $d_{\underline{2}} \in C^{N}(\underline{2})$ e $(X, \xi) \in C^{N}$ [Top] a aplicação $\xi\left(d_{2}\right)$ define uma estrutura de $\mathcal{H}$-espaço homotopicamente associativo em $X$.

Definição 5.1.3. Um $\mathcal{H}$-espaço $X$ é admissível se for homotopicamente associativo e se $\mu\left(x \times 1_{X}\right) \simeq$ $\mu\left(1_{X} \times x\right)$ para todo $x \in \mathrm{Top}_{*}(* \sqcup *, X) \cong X$.

A estrutura de $k$-álgebra graduada de $H_{\bullet}(X ; k)$ para um $\mathcal{H}$-espaço admissível $X$ é comutativa.

Para $1<N \leq \infty$ as estruturas de $\mathcal{H}$-espaço nos $C^{N}$-espaços são admissíveis.

Definição 5.1.4. Um $\mathcal{H}$-espaço homotopicamente associativo $X$ é grouplike se o monoide $\left(\pi_{0} X, \mu_{*}\right.$, $\left.\left[X_{*}\right]\right)$ for um grupo.

As estruturas de $\mathcal{H}$-espaços nos espaços de $N$-laços induzidos das estruturas de $C^{N}$-álgebras são grouplike.

Para $\left(X_{c}, X_{o}\right) \in \mathcal{S} C^{N}[\mathrm{Top}]$ temos que $X_{c}$ é uma $C^{N}$-álgebra e $X_{o}$ é uma $C^{N-1}$-álgebra. Para $N>1$ dizemos que $\left(X_{c}, X_{o}\right)$ é grouplike se a estrutura de $\mathcal{H}$-espaço em $X_{c}$ e $X_{o}$ são grouplike. 
Definição 5.1.5. Um completamento de grupo homológico de um $\mathcal{H}$-espaço admissível $X$ é um $H$-espaço admissível grouplike $G$ equipado com uma $\mathcal{H}$-aplicação $g \in \mathcal{H}-\mathrm{Top}(X, G)$ tal que para todo anel comutativo $k$ o homomorfismo induzido $\bar{g}_{*} \in k-\operatorname{AlgGr}\left(H_{\bullet}(X, k)\left[\pi_{0} X^{-1}\right], H_{\bullet}(G, k)\right)$ é um isomorfismo.

Para todo $\mathcal{H}$-espaço grouplike $X$ temos uma equivalência homotópica entre $X$ e $X^{e} \times \pi_{0} X$, aonde $X^{e}$ é a componente conexa de $X$ que contém $e$ [CLM76, Lemma I.4.6]. Como todo $\mathcal{H}$ espaço $X$ é simples, i.e. $\pi_{1} X$ é abeliano e age trivialmente em $\pi_{q} X$ para todo $q \in \mathbb{N}$, o teorema dual de Whitehead [Ma83] para espaços simples implica que um completamento de grupo homológico de um $\mathcal{H}$-espaço admissível grouplike é uma equivalência fraca.

Teorema 5.1.6. [Teorema de aproximação] Se $X \in T o p_{*}$ é conexo então $\alpha_{X}^{N}$ é uma equivalência fraca. Se $1<N \leq \infty$ então $\alpha_{X}^{N}$ é um completamento de grupo homologico para todo $X \in T_{0} p_{*}$.

Corolário 5.1.7. Se $X \in C^{N}$ [Top] é conexo, então $\alpha_{X}^{N}$ é uma equivalência fraca. Se $1<N \leq \infty$ e $X \in C^{N}\left[\right.$ Top] é grouplike então $\alpha_{X}^{N}$ é uma equivalência fraca.

Provaremos agora uma versão relativa desse corolário. Defina $\alpha_{2}^{N}$ como a composição das seguintes transformações naturais:

$$
S C^{N} \stackrel{S C^{N} \eta_{2}^{N}}{\Longrightarrow} S C^{N} \Omega_{2}^{N} \Sigma_{2}^{N} \stackrel{\theta_{\Sigma}^{N}}{\Longrightarrow} \Omega_{2}^{N} \Sigma_{2}^{N}
$$

que é explicitamente dada por

$$
\begin{gathered}
\alpha_{c\left(X_{c}, X_{o}\right)}^{N}\left(\left[d_{\underline{n}}, x^{\underline{n}}\right]\right)(t)= \begin{cases}{\left[x^{j}, d_{j}^{-1}(t)\right],} & t \in d_{j}\left(I^{N}\right) \\
*, & t \notin d_{\underline{n}}\left(\coprod_{\underline{n}} I^{N}\right)\end{cases} \\
\begin{cases}\alpha_{o\left(X_{c}, X_{o}\right)}^{N}\left(\left[d_{\underline{n, m},}, x^{n, m}\right]\right)(s)= \\
{\left[x^{i}, d_{i}^{-1}(0, s)\right], s^{\prime} \mapsto\left\{\begin{array}{ll}
{\left[x^{a}, d_{a}^{-1}\left(s^{\prime}, s\right)\right],} & \left(s^{\prime}, s\right) \in d_{a}\left(I^{N}\right), a \in \underline{n, m} \\
*, & \left(s^{\prime}, s\right) \notin d_{\underline{n, m}}\left(\coprod_{\underline{n, m}} I^{N}\right)
\end{array}\right),} & (0, s) \in d_{i}\left(I^{N}\right), i \in \underline{m} \\
*, s^{\prime} \mapsto\left\{\begin{array}{ll}
{\left[x^{j}, d_{j}^{-1}\left(s^{\prime}, s\right)\right],} & \left(s^{\prime}, s\right) \in d_{j}\left(I^{N}\right), j \in \underline{n} \\
*, & \left(s^{\prime}, s\right) \notin d_{\underline{n, m}}\left(\coprod_{\underline{n, m}} I^{N}\right)
\end{array}\right) \notin d_{\underline{m}}\left(\coprod_{\underline{m}} I^{N}\right)\end{cases}
\end{gathered}
$$

Note que $\alpha_{c\left(X_{c}, X_{o}\right)}^{N}=\Omega^{N} i_{\Sigma^{N} X_{c}} \alpha_{X_{c}}^{N} \operatorname{com} i_{\Sigma^{N} X_{c}} \in \operatorname{Top}_{*}\left(\Sigma^{N} X_{c}, \Sigma^{N-1}\left(X_{o} \wedge I\right) \vee \Sigma^{N} X_{c}\right)$ a inclusão de $\Sigma^{N} X_{c}$ na soma wedge, que é um retrato por deformação e portanto uma equivalência fraca.

Sejam $\pi_{c}, \pi_{o}: \mathrm{Top}_{*}^{2} \rightarrow \mathrm{Top}_{*}$ as projeções nas componente fechada e aberta respectivamente.

Os funtores $S C_{c}^{N}:=\pi_{c} S C^{N}$ e $S C_{o}^{N}:=\pi_{o} S C^{N}$ são $S C^{N}$-funtores, pois podemos definir $\lambda^{S C_{o}^{N}}:=$ $\pi_{o} \mu^{S C^{N}}$ e $\lambda^{S C_{c}^{N}}:=\pi_{c} \mu^{S C^{N}}$. O funtor $C^{N-1} \pi_{o}$ também é um $S C^{N}$-funtor. A aplicação natural estrutural 
$\lambda^{C^{N-1} \pi_{o}}: C^{N-1} \pi_{o} S C^{N}=C^{N-1} S C_{o}^{N} \Rightarrow C^{N-1} \pi_{o}$ é dada por:

$$
\lambda_{\left(X_{c}, X_{o}\right)}^{C^{N-1} \pi_{o}}\left(\left[d_{\underline{m}},\left(\left[d_{n^{i}, m^{i}}, x^{n^{i}, m^{i}}\right]\right)_{i \in \underline{m}}\right]\right):=\left[\mu_{\underline{m},\left(\underline{m}^{i}\right)_{i \in \underline{m}}}^{C^{N-1}}\left(d_{\underline{m}},\left(d_{\underline{m}^{i}} \uparrow_{\{0\} \times I^{N-1}}\right)_{i \in \underline{m}}\right), x^{\sum_{i=1}^{m} m^{i}}\right]
$$

Também podemos definir o morfismo de $S C^{N}$-funtores $p_{o}^{N}: \pi_{o} S C^{N} \Rightarrow C^{N-1} \pi_{o}$ por

$$
p_{o\left(X_{c}, X_{o}\right)}^{N}\left(\left[d_{\underline{n, m}}, x^{n, m}\right]\right):=\left[d_{\underline{m}} \Upsilon_{\{0\} \times I^{N-1}}, x^{\underline{m}}\right]
$$

Note que $\pi_{o} S C^{N}\left(X_{o}, X_{c}\right)$ é uma $C^{N-1}$-álgebra e que $p_{o}^{N}$ é uma $C^{N-1}$-aplicação natural, e portanto para $N>1$ é uma $\mathcal{H}$-aplicação natural e se $N>2$ é uma $\mathcal{H}$-aplicação natural entre $\mathcal{H}$-espaços admissíveis. Provamos agora que $p_{o}^{N}$ é uma quasi-fibração em pares $\left(X_{c}, X_{o}\right) \operatorname{com} X_{o}$ bem pontuado.

Definição 5.1.8. Uma aplicação $p \in \operatorname{Top}(E, B)$ é uma quasi-fibração se as inclusões naturais $i_{p^{-1}(b)} \in \operatorname{Top}\left(p^{-1}(b),\left\{(e, \gamma) \in E \times_{B} B^{I} \mid \gamma(0)=b, \gamma(1)=e\right\}\right)$ dadas por $i_{p^{-1}(b)}(f)=(f, t \mapsto p(f))$ são equivalências fracas para todo $b \in B$.

Um subespaço $U \subset B$ é distinguido se $p \uparrow_{p^{-1}(U)} \in \operatorname{Top}\left(p^{-1}(U), U\right)$ é uma quasi-fibração.

De [Ma90, 2.7] temos o seguinte critério para uma aplicação ser uma quasi-fibração.

Proposição 5.1.9. Seja $p \in T o p(E, B)$ uma aplicação de espaços filtrados tal que $F^{k} E=p^{-1} F^{k} B$ para cada $k \in \mathbb{N}$. Se para cada $k>0$ a aplicação $p \uparrow_{F^{k} E}$ é obtido como um pushout de quadrados comutativos $\left(g_{k}, f_{k}\right) \in \operatorname{Top}^{\rightarrow}\left(q_{k}, p \uparrow_{F^{k-1} E}\right) e\left(j_{k}, i_{k}\right) \in \operatorname{Top} \rightarrow\left(q_{k}, p_{k}\right) \operatorname{com} q_{k} \in \operatorname{Top}\left(D_{k}, A_{k}\right)$ e $p_{k} \in$ $\operatorname{Top}\left(E_{k}, B_{k}\right)$

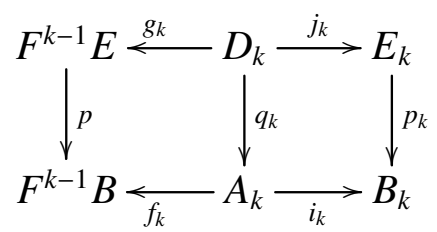

que satisfazem as seguintes condições:

i) $F^{0} B$ é distinguido;

ii) $p_{k}$ é uma fibração;

iii) $i_{k}$ e $j_{k}$ são inclusões de pares-RDV;

iv) $\left(j_{k}, i_{k}\right)$ é um pullback;

v) $g_{k} \Gamma_{q_{k}^{-1}(a)} \in \operatorname{Top}\left(q_{k}^{-1}(a), p^{-1}\left(f_{k}(a)\right)\right)$ é uma equivalência fraca para todo $a \in A_{k}$;

então cada $F^{k} B$ é distinguido e p é uma quasi-fibração.

Teorema 5.1.10. Seja $\left(X_{c}, X_{o}\right) \in T o p_{*}^{2}$. Se $X_{o}$ é bem pontuado $p_{o\left(X_{c}, X_{o}\right)}^{N}$ é uma quasi-fibração com fibra $C^{N}\left(X_{c}\right)$. 
Demonstração: Mostraremos que $p_{o\left(X_{c}, X_{o}\right)}^{N}$ e as filtrações naturais de $C^{N-1} X_{o}$ e $\mathcal{S C}^{N}\left(X_{c}, X_{o}\right)$ do capítulo 4 satisfazem as condições da proposição 5.1.9.

Fixe $k \in \mathbb{N}$. Seja $\mathbb{S}_{\underline{k}}$ a subcategoria plena de $\mathbb{S}$ contendo apenas $\underline{k}$ e $2 \mathbb{S}_{\underline{k}}$ a subcategoria plena de $2 \mathbb{S}_{\text {inj }}$ contendo apenas os 2 -conjuntos da forma $\underline{n, k}$. Podemos definir

$$
C_{k}^{N-1} X_{o}:=\int^{\mathbb{S}_{\underline{k}}} C^{N-1}(\underline{k}) \times X_{o}^{\underline{k}}
$$

$\mathrm{e}$

$$
\operatorname{SC}_{k}^{N}\left(X_{c}, X_{o}\right):=\int^{2 \mathbb{S}_{\underline{k}}} \mathcal{S} C^{N}(\underline{n, k}) \times\left(X_{c}, X_{o}\right)^{n, k}
$$

Definimos também

$$
A_{k}^{N}:=\left\{\left[d_{\underline{k}}, x^{\underline{k}}\right] \in C_{k}^{N-1} X_{o} \mid \exists i \in \underline{k} \text { t.q. } x^{i}=X_{o *}\right\}
$$

$\mathrm{e}$

$$
D_{k}^{N}:=\left\{\left[d_{\underline{n, k}}, x^{n, k}\right] \in S C_{k}^{N}\left(X_{c}, X_{o}\right) \mid \exists i \in \underline{k} \text { t.q. } x^{i}=X_{o *}\right\}
$$

As aplicações $p_{o\left(X_{c}, X_{o}\right)}^{N} \mathrm{Top}_{*}\left(F^{k} \pi_{o} S C\left(X_{c}, X_{o}\right), F^{k} C^{N-1} X_{o}\right)$ são pushouts em Top $\mathrm{p}_{*}^{\rightarrow}$ das aplicações no seguinte diagrama:

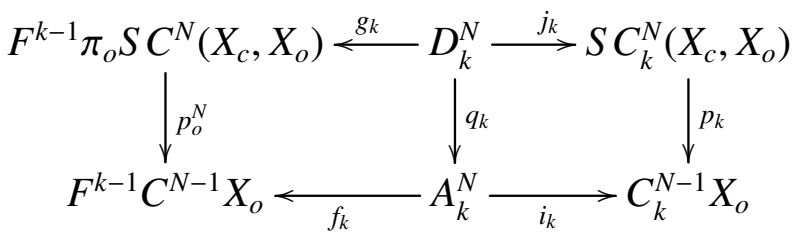

no qual $i_{k}$ e $j_{k}$ são inclusões de subespaços, $g_{k}$ e $f_{k}$ são induzidos das degenerações dos pequenos cubos $d_{i}$ cujos índices são os mesmos que os dos $x^{i}$ iguais ao ponto base e $q_{k}$ e $p_{k}$ são definidos da mesma forma que $p_{o}^{N}$. Essas aplicações satisfazem as condições da proposição 5.1.9:

i) $F^{0} C^{N-1} X_{o}=\{*\}$. Como todo espaço é fibrante e toda fibração é uma quasi-fibração $F^{0} C^{N-1} X_{o}$ é distinguida;

ii) Por definição $p_{k}$ é uma fibração se para todo quadrado comutativo $\left(\left[d_{l, k}, x^{l, k}\right],\left[\delta_{\underline{k}}, \xi^{k}\right]\right) \in$ $\operatorname{Top} \rightarrow\left(j^{q}, p_{k}\right)$ admite um levantamento $\tilde{H} \in \operatorname{Top}\left(I^{q+1}, S C_{k}^{N}\left(X_{c}, X_{o}\right)\right)$.

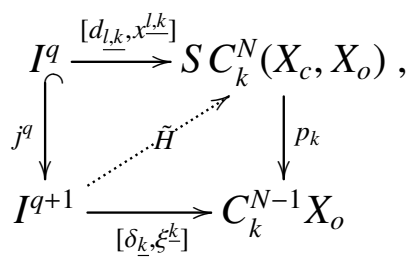

Lembre do capítulo 4 que um pequeno cubo $d_{a}$ em $S C^{\mathcal{N}}(n, m)$ ou $C^{\mathcal{N}}(\underline{m})$ é da forma $d_{a}(s)=$ $M_{a} s+C_{a}$ para alguma matriz diagonal $M_{a}$ com entradas positivas e algum $C_{a} \in I^{N}$. Logo para qualquer $\left(d_{\underline{n, m},}, \delta_{\underline{m}}\right) \in \mathcal{S} C^{N}\left(\underline{n, m)} \times_{C^{N-1}(\underline{m})} C^{N-1}(\underline{m})^{I}\right.$ e $v \in(0,1]$ podemos definir $\gamma_{d_{n, m}, \delta_{\underline{m}}}^{v} \in$ 
$\operatorname{Top}\left(I,\left[\sqcup_{a \in n, m} I_{a}^{N}, I^{N}\right]\right) \mathrm{com}$

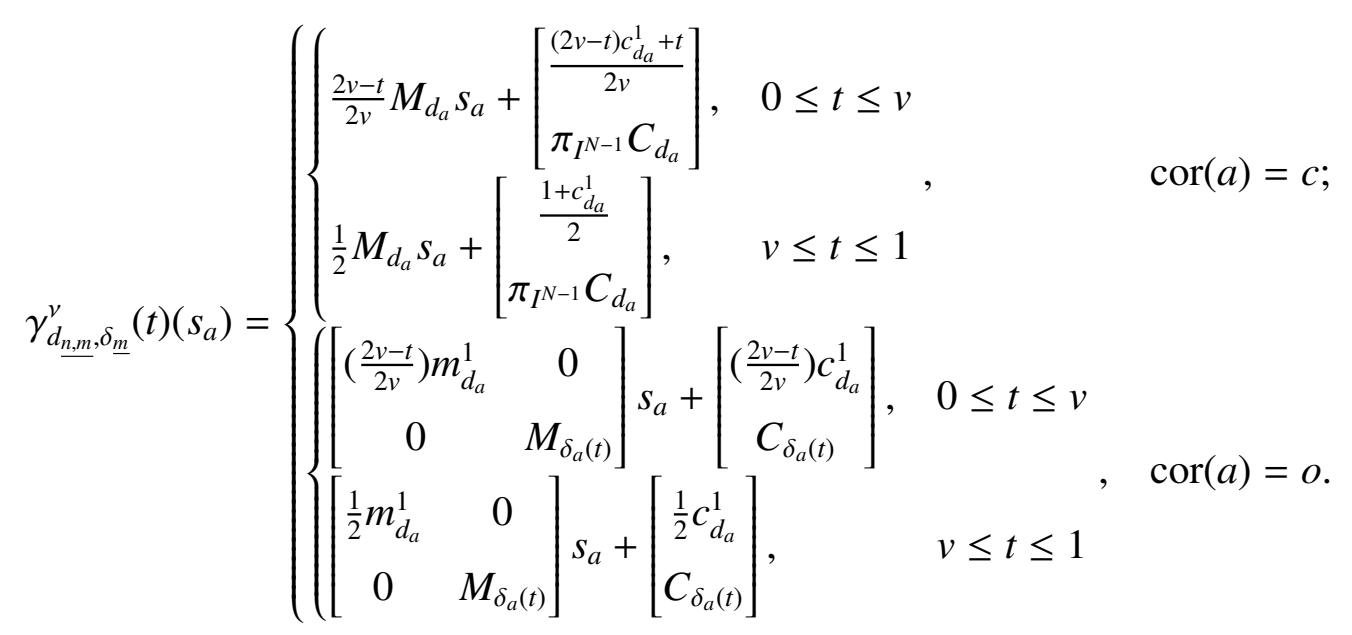

i.e. $\gamma_{d_{n, m}, \delta_{\underline{m}}}^{v}$ é o caminho em $\left[\sqcup_{a \in n, m} I_{a}^{N}, I^{N}\right]$ tal que $\gamma_{d_{n, m}, \delta_{\underline{m}}}^{v}(0)=d_{\underline{n, m}}, p_{o} \gamma_{d_{n, m}, \delta_{\underline{m}}}^{v}=\delta_{\underline{n}}$, a altura na primeira coordenada dos pequenos cubos abertos é linearmente diminuída até ter a metade da altura original no intervalo $[0, v]$ e se mantém constante no intervalo $[v, 1]$, e tal que os comprimentos dos lados em todas as coordenadas dos pequenos cubos fechados e a distância na primeira coordenada do centro dos pequenos cubos fechados e 1 também são linearmente divididos pela metade no intervalo $[0, v]$ e se mantém constantes no intervalo $[v, 1]$.

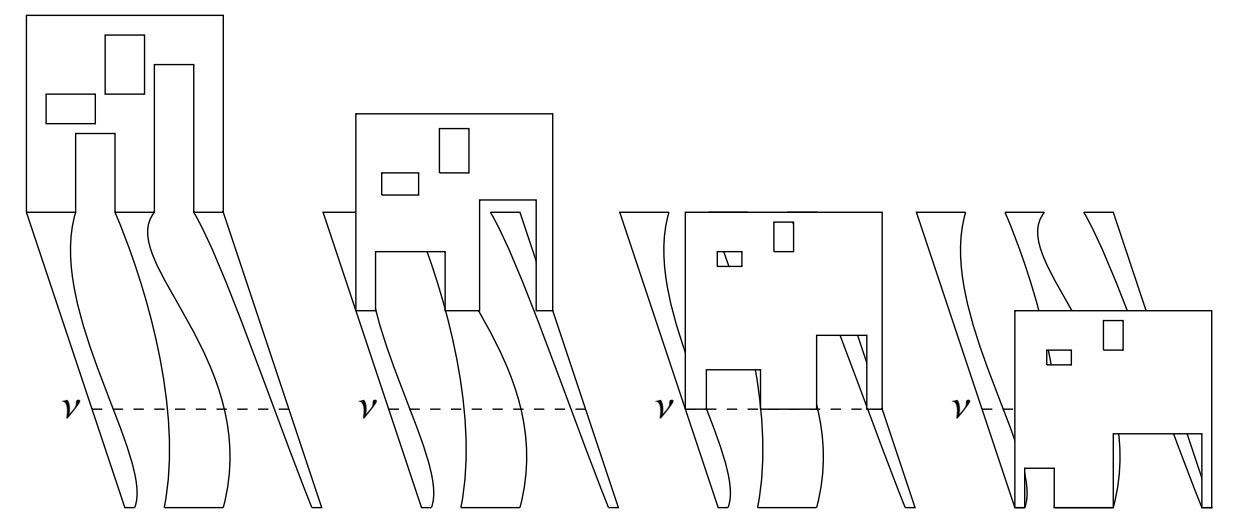

Para alguns $\left(d_{\underline{n, m}, m}, \delta_{\underline{m}}\right)$ e $v$ pode ser que $\gamma_{d_{n, m}, \delta_{m}}^{v} \notin \mathcal{S C}(\underline{n, m})^{I}$, pois pode haver $a, a^{\prime} \in \underline{n, m}$ e $t \in I$ tais que $a \neq a^{\prime}$ e $\gamma_{d_{n, m}, \delta_{\underline{m}}, a}^{v}(t)\left(I^{N}\right) \cap \gamma_{d_{n, m}, \delta_{\underline{m}}, a^{\prime}}^{v}(t)\left(I^{N}\right) \neq \emptyset$, mas sempre existe um $v^{\prime} \in(0,1]$ tal que para $v \in\left(0, v^{\prime}\right]$ essas intersecções são vazias. Podemos então definir para cada $\underline{n, m} \in 2 \mathbb{S}$ a aplicação $v_{n, m} \in \operatorname{Top}\left(S C^{N}(\underline{n, m}) \times_{C^{N-1}(\underline{m})} C^{N-1}(\underline{m})^{I},(0,1]\right)$ definida por

$$
v_{n, m}\left(d_{n, m}, \delta_{\underline{m}}\right)=\max \left\{v \in(0,1] \mid \gamma_{d_{n, m}, \delta_{\underline{m}}}^{v} \in \mathcal{S C}^{N}(\underline{n, m})^{I}\right\}
$$

Provamos agora que para qualquer quadrado comutativo $\left(\left[d_{\underline{l, k},}, x^{l, k}\right],\left[\delta_{\underline{k}}, \xi^{\underline{k}}\right]\right)$ como acima existe um $\bar{v} \in(0,1]$ tal que $\gamma_{d_{\underline{\underline{\nu}},}^{\bar{v}}(s), \delta_{\underline{\underline{k}}}(s)} \in \mathcal{S} C^{N}(\underline{n, m})^{I}$ para todo $s \in I^{q}$.

Seja $F^{l} 2 \mathbb{S}_{k}$ a subcategoria plena de $2 \mathbb{S}_{k}$ contendo apenas os 2-conjuntos da forma $\underline{n, k}$ com $n \leq l$. As inclusões naturais de $\int^{F^{l} \mathbb{S}_{k}} \mathcal{S} C^{N}(\underline{n, k}) \times\left(X_{c}, X_{o}\right)^{n, k}$ em $S C_{k}^{N}\left(X_{c}, X_{o}\right)$ nos dão uma 
filtração $F^{l} S C_{k}^{N}\left(X_{c}, X_{o}\right)$.

Qualquer subespaço compacto $K$ de $S C_{k}^{N}\left(X_{c}, X_{o}\right)$ está contido em $F^{l} S C_{k}^{N}\left(X_{c}, X_{o}\right)$ para algum $l \in \mathbb{N}$. Para ver isso assuma que existe uma sequência infinita de pontos $\left(z_{i}\right)_{i \in \mathbb{N}} \in \prod_{i \in \mathbb{N}} K$ todos contidos em $F^{l_{i}} S C_{k}^{N}\left(X_{c}, X_{o}\right) \backslash F^{l_{i}-1} S C_{k}^{N}\left(X_{c}, X_{o}\right)$ distintos. Considere o subconjunto $S$ desses pontos de $K$. Para mostrar que $S$ é fechado assuma que $S \cap F^{l-1} S C_{k}^{N}\left(X_{c}, X_{o}\right)$ é fechado. Então $S \cap F^{l} S C_{k}^{N}\left(X_{c}, X_{o}\right)$ contém no máximo mais um ponto. O espaço $S C_{k}^{N}\left(X_{c}, X_{o}\right)$ é fracamente Hausdorff, logo pontos são fechados e portanto $S \cap F^{l} S C_{k}^{N}\left(X_{o}, X_{c}\right)$ é fechado. O mesmo argumento mostra que qualquer subconjunto de $S$ é fechado, logo $S$ tem a topologia discreta. Como $S$ é um subespaço fechado de um compacto ele deve ser compacto e portanto finito, uma contradição. ${ }^{1}$

A filtração $F^{l} S C_{k}^{N}\left(X_{c}, X_{o}\right)$ e a aplicação $\left[d_{l, k}, x^{l, k}\right]$ do quadrado comutativo induzem uma filtração $F^{l} I^{q}:=\left[d_{l, k}, x^{l, k}\right]^{-1}\left(F^{l} S C_{k}^{N}\left(X_{c}, X_{o}\right)\right)$. Definimos então $v^{l} \in \operatorname{Top}\left(\overline{F^{l} I^{q}-F^{l-1} I^{q}},(0,1]\right)$ por

$$
v^{l}(s)=v_{l, k}\left(d_{\underline{k l}}(s), \delta_{\underline{k}}(s)\right)
$$

Como $I^{q}$ é compacto $\overline{F^{l} I^{q}-F^{l-1} I^{q}}$ é compacto, e portanto as imagens dos $v^{l}$ tem um mínimo positivo.

Pelas duas últimas observações podemos definir

$$
\bar{v}:=\min \left\{v^{l}(s) \mid l \in \mathbb{N} ; s \in \overline{F^{l} I^{q}-F^{l-1} I^{q}}\right\}
$$

Isso nos dá uma aplicação $\tilde{H} \in \mathrm{T} \circ \mathrm{p}\left(I^{q+1}, S C_{k}^{N}\left(X_{c}, X_{o}\right)\right)$ definida por

$$
\tilde{H}(s, t):=\left[\gamma_{d_{\underline{l, k}}^{\bar{v}}(s), \delta_{\underline{k}}(s)}^{\bar{x}}(t),\left(x^{l}(s), \xi^{k}(s, t)\right)\right]
$$

que é um levantamento de $\left(\left[d_{\underline{l, k}}, x^{l, k}\right],\left[\delta_{\underline{k}}, \xi^{\underline{k}}\right]\right)$.

iii) Como $X_{o}$ é bem pontuado temos aplicações $u \in \operatorname{Top}\left(X_{o}, I\right)$ e $H \in \operatorname{Top}\left(X_{o} \times I, X_{o}\right)$ que nos dão que $\left(X_{o *}, X_{o}\right)$ um par-RDV, e portanto podemos definir aplicações $u^{\prime} \in \operatorname{Top}\left(C_{k}^{N-1} X_{o}, I\right)$ como $u^{\prime}\left(\left[d_{\underline{k}}, x^{\underline{k}}\right]\right):=\min \left\{u\left(x^{i}\right) \mid i \in \underline{k}\right\}$ e $H^{\prime} \in \operatorname{Top}\left(C_{k}^{N-1} X_{o} \times I, C_{k}^{N-1} X_{o}\right) \operatorname{como} H^{\prime}\left(\left[d_{\underline{k}}, x^{\underline{k}}\right], t\right):=$ $\left[d_{\underline{k}},\left(H\left(x^{i}, t\right)\right)_{i \in \underline{k}}\right]$ que nos dão que $\left(A_{k}^{N}, C_{k}^{N-1} X_{o}\right)$ é um par-RDV. Que $\left(D_{k}^{N}, S C_{k}^{N}\left(X_{o}, X_{c}\right)\right)$ é um par-RDV segue de um argumento análogo.

iv) É trivial checar que $\left(j_{k}, i_{k}\right)$ é um pullback.

v) Fixe $\left[d_{\underline{k}}, x^{\underline{k}}\right] \in A_{k}^{N}$ e defina os subespaços

$$
P_{k}^{N}:=\left\{\begin{array}{l|l}
{\left[d_{\underline{n, k}}, x^{n, k}\right] \in q_{k}^{-1}\left(\left[d_{\underline{k}}, x^{\underline{k}}\right]\right)} & \begin{array}{l}
\forall j \in \underline{n}, d_{j}(I) \subset\left[\frac{1}{2}, 1\right] \times I^{N-1} ; \\
\forall i \in \underline{k}, d_{i}(I) \subset\left[0, \frac{1}{2}\right] \times I^{N-1} .
\end{array}
\end{array}\right\}
$$

\footnotetext{
${ }^{1} \mathrm{O}$ argumento nesse parágrafo foi providenciado por Eduardo Hoefel via comunicação privada.
} 
$\mathrm{e}$

$$
Q_{k}^{N}:=\left\{\begin{array}{l|l}
{\left[d_{\underline{n, k-1}}, x^{n, k-1}\right] \in p_{o}^{-1}\left(f_{k}\left(\left[d_{\underline{k}}, x^{\underline{k}}\right]\right)\right)} & \begin{array}{l}
\forall j \in \underline{n}, d_{j}(I) \subset\left[\frac{1}{2}, 1\right] \times I^{N-1} ; \\
\forall i \in \underline{k-1}, d_{i}(I) \subset\left[0, \frac{1}{2}\right] \times I^{N-1} .
\end{array}
\end{array} .\right.
$$

Então $P_{k}^{N}$ e $Q_{k}^{N}$ são retratos por deformação de $q_{k}^{-1}\left(\left[d_{\underline{k}}, x^{\underline{k}}\right]\right)$ e $p_{o}^{N-1}\left(f_{k}\left(\left[d_{\underline{k}}, x^{k}\right]\right)\right)$ respectivamente, e a restrição $g_{k} \Gamma_{P_{k}^{N}} \operatorname{Top}\left(P_{k}^{N}, Q_{k}^{N}\right)$ éuma fibração com fibra contrátil, e portanto uma equivalência fraca. Isso implica que $\left.\left.g_{k} \uparrow_{q_{k}^{-1}\left(\left[d_{\underline{k}}, x^{k}\right]\right.}\right]\right) \operatorname{Top}\left(q_{k}^{-1}\left(\left[d_{\underline{k}}, x^{\underline{k}}\right]\right), p_{o}^{-1}\left(f_{k}\left(\left[d_{\underline{k}}, x^{\underline{k}}\right]\right)\right)\right)$ também é uma equivalência fraca.

Logo pela proposição 5.1.9 $p_{o\left(X_{c}, X_{o}\right)}^{N}$ é uma quasi-fibração. Que $C^{N} X_{c}$ é a fibra de $p_{o\left(X_{c}, X_{o}\right)}^{N}$ segue facilmente das definições.

Corolário 5.1.11. Seja $\left(X_{c}, X_{o}\right) \in \mathcal{S} C^{N}[$ Top $] \operatorname{com} X_{o} \in \operatorname{Top}_{\text {Cof. }}$ Então $\alpha_{2\left(X_{c}, X_{o}\right)}^{N}$ é uma equivalência fraca se $\left(X_{c}, X_{o}\right)$ forem conexos e um completamento de grupo se $2<N \leq \infty$.

Demonstração: Temos o seguinte diagrama comutativo:

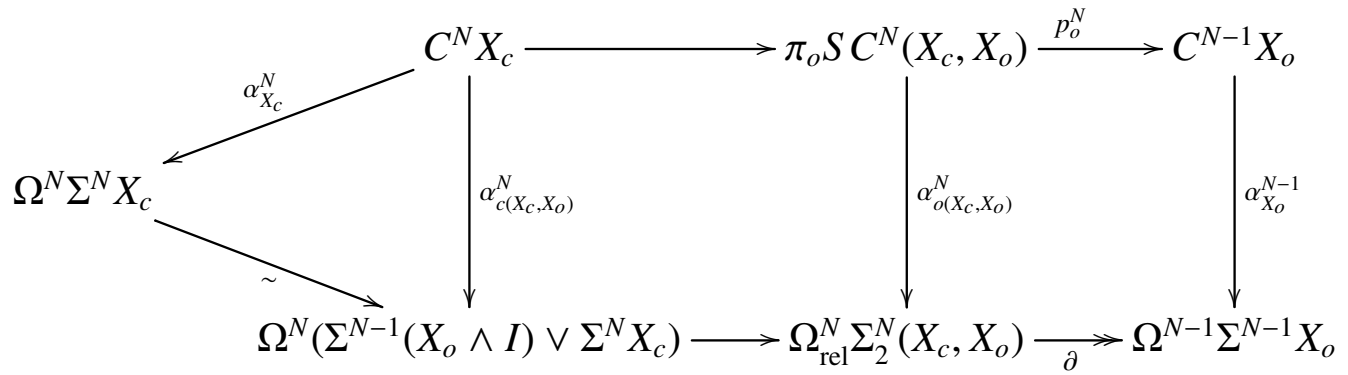

Se $2<N \leq \infty$ este é um diagrama comutativo de $\mathcal{H}$-aplicações entre $\mathcal{H}$-espaços admissíveis. O teorema 5.1.6 nos dá que $\alpha_{X_{c}}^{N}$ e $\alpha_{X_{o}}^{N-1}$ são completamentos de grupo, e portanto $\alpha_{c\left(X_{c}, X_{o}\right)}^{N}$ também é. Temos que $H_{\bullet}\left(\pi_{o} S C^{N}\left(X_{c}, X_{o}\right)\right) \cong H_{\bullet}\left(C^{N} X_{c}\right) \otimes H_{\bullet}\left(C^{N-1} X_{o}\right)$. Além disso $\pi_{1}\left(C^{N-1} X_{o}\right)$ age trivialmente em $H_{\bullet}\left(C^{N} X_{c}\right)$ e $\pi_{1}\left(\Omega^{N-1} \Sigma^{N-1} X_{o}\right)$ age trivialmente em $H_{\bullet}\left(\Omega^{N} \Sigma^{N} X_{c}\right)$, logo pela sequência espectral de Serre das fibrações (por $X_{o} \in$ Top ${ }_{\text {Cof }}$ e [FP90, Cor.5.3.7] temos que $\Omega^{N-1} \Sigma^{N-1} X_{o}$ tem o tipo homotópico de um CW-complexo, e portanto a sequência espectral está bem definida) temos que $\alpha_{o\left(X_{c}, X_{o}\right)}^{N}$ é um completamento de grupo.

A demonstração para a condição de $\left(X_{c}, X_{o}\right)$ segue da seuqencia exata longa de grupos de homotopia.

\subsection{Compatibilidade da realização geométrica}

As propriedade de compatibilidade do funtor de realização geométrica dessa sessão são análogas aos resultados em [Ma72, S. 12]. Damos apenas um esboço das demonstrações pois elas são simples adaptações dos argumentos dados lá.

Proposição 5.2.1. Existem homeomorfismos naturais $\tau^{N} \in \operatorname{Top}_{*}^{\rightarrow}\left(\left|\Sigma_{2}^{N\langle\bullet\rangle}\left(X_{c}, X_{o}\right)^{\langle\bullet\rangle}\right|, \Sigma_{2}^{N}\left|\left(X_{c}, X_{o}\right)^{\langle\bullet\rangle}\right|\right)$ para $N<\infty e \tau^{\infty} \in S p^{\nearrow}\left(\left|\Sigma_{2}^{\infty\langle\bullet\rangle}\left(X_{c}, X_{o}\right)^{\langle\bullet\rangle}\right|, \Sigma_{2}^{\infty}\left|\left(X_{c}, X_{o}\right)^{\langle\bullet\rangle}\right|\right)$. 


\section{Demonstração: Definimos}

$$
\tau^{N}([[x, s], u]):=[[x, u], s]
$$

e checamos diretamente que isso define um homeomorfismo.

Proposição 5.2.2. Seja $Q \in 2 O p(T O p)$ e $Q$ sua monada em $\mathrm{Top}_{*}^{\{o, c\}}$ associada. Então existe um homeomorfismo natural $v^{Q} \in \operatorname{Top}_{*}^{\{o, c\}}\left(\left|Q^{\langle\bullet\rangle}\left(X_{c}, X_{o}\right)^{\langle\bullet\rangle}\right|, Q\left|\left(X_{c}, X_{o}\right)^{\langle\bullet\rangle}\right|\right)$ tais que os seguintes diagramas comutam:
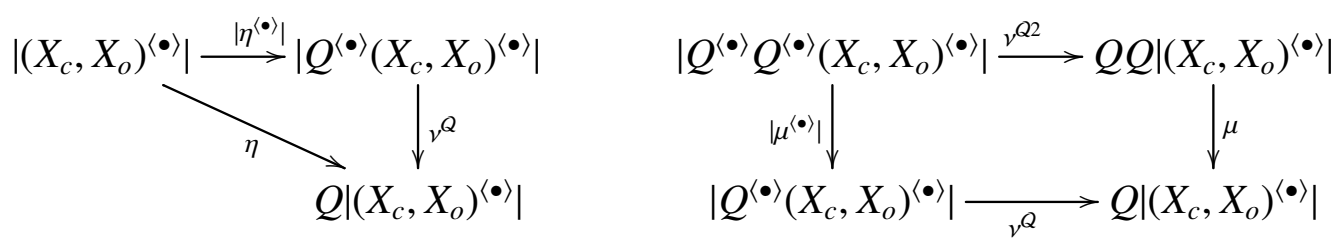

$\operatorname{Se}\left(X_{c}, X_{o}\right)^{\langle\bullet\rangle} \in Q[\text { Top }]^{\Lambda^{o p}}$ então $\left|\left(X_{c}, X_{o}\right)^{\langle\bullet\rangle}\right| \in Q[$ Top $]$, logo realização geométrica define um funtor $Q[T o p]^{\Delta^{o p}} \rightarrow Q[T o p]$.

Demonstração: Definimos

$$
v^{Q}\left(\left[\left[q_{S}, x^{S}\right], u\right]\right)=\left[q_{S},\left(\left[x^{a}, u\right]\right)_{a \in S}\right]
$$

e verificamos diretamente pelas definições que é um homeomorfismo e os diagramas comutam. A segunda afirmação então segue da primeira.

Definição 5.2.3. Um espaço relativo simplicial $\iota^{\langle\bullet\rangle}: B^{\langle\bullet\rangle} \rightarrow Y^{\langle\bullet\rangle} \in\left(\mathrm{Top}_{*}\right)^{\Delta^{o p}}$ é próprio se for cofibrante na estrutura modelo de Strøm. Um espectro relativo simplicial $\iota_{\bullet}^{\langle\bullet\rangle}: B_{\bullet}^{\langle\bullet\rangle} \rightarrow Y_{\bullet}^{\langle\bullet\rangle} \in$ $\left(\mathrm{Sp}^{\nearrow}\right)^{\Delta^{\mathrm{op}}}$ é próprio se for cofibrante na estrutura modelo de Strøm.

Proposição 5.2.4. Para $1 \leq N<\infty e \iota^{\langle\bullet\rangle}: B^{\langle\bullet\rangle} \rightarrow Y^{\langle\bullet\rangle} \in\left(T \circ p_{*}^{\rightarrow}\right)^{\Delta^{o p}}$ um espaço relativo simplicial próprio com cada $\iota^{\langle q\rangle} N-1$-conexo temos uma equivalência fraca $\gamma_{2}^{N} \in \operatorname{Top}_{*}^{2}\left(\left|\Omega_{2}^{N\langle\bullet\rangle} \iota^{\langle\bullet\rangle}\right|, \Omega_{2}^{N}\left|\iota^{\langle\bullet\rangle}\right|\right)$.

Para $\iota_{\bullet}^{\langle\bullet\rangle}: B_{\bullet}^{\langle\bullet\rangle} \rightarrow Y_{\bullet}^{\langle\bullet\rangle} \in\left(S P^{\nearrow}\right)^{\Delta^{o p}}$ um espectro relativo simplicial próprio com cada $\iota_{p}^{\langle q\rangle} p-1$ conexo temos uma equivalência fraca $\left.\gamma_{2}^{\infty} \in \operatorname{Top}_{*}^{2}\left(\left|\Omega_{2}^{\infty}\langle\bullet\rangle\left\langle\iota_{\bullet}\right\rangle, \Omega_{2}^{\infty}\right| \iota_{\bullet}\right\rangle \mid\right)$.

Demonstração: Para $N<\infty$ definimos

$$
\begin{gathered}
\gamma_{c}^{N}([\alpha, u])(t):=[\alpha(t), u] ; \\
\gamma_{o}^{N}([(\beta, \gamma), u])(s):=\left[\left(\beta(s), s^{\prime} \mapsto \gamma(s)\left(s^{\prime}\right)\right), u\right] .
\end{gathered}
$$

Essa é uma aplicação de fibras de uma quasi-fibração e uma fibração de espaços de caminhos simpliciais sobre o mesmo espaço cujos espaços totais são contrateis, e a contração é compatível com a estrutura simplicial. Isso nos dá que $\gamma_{2}^{N}$ são equivalências fracas. A aplicação $\gamma_{2}^{\infty}$ é dada como um colimite dos $\gamma_{2}^{N}$ para $N<\infty$. 
Proposição 5.2.5. Para $1 \leq N \leq \infty$ as aplicações $\gamma_{2}^{N}$ são $S C^{N}$-aplicações e o diagrama a seguir é comutativo:

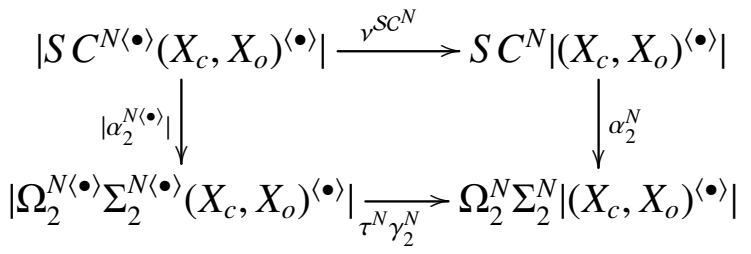

Demonstração: Com as definições dos morfismo dadas checamos diretamente a comutatividade do diagrama dado e os que nos dão as estruturas de $\mathcal{S} C^{N}$-aplicações

\subsection{Principio de reconhecimento de espaços de laços finitos}

Nessa sessão assumimos que $N<\infty$.

Definição 5.3.1. Seja $\pi_{2}^{N} \in 2 O p(\mathrm{Top})\left(\mathcal{D}^{N}, \mathcal{S} C^{N}\right)$ uma equivalência fraca entre 2-operads $2 \mathbb{S}$ livres. O funtor de desenlaçamento para $\mathcal{D}^{N}$-álgebras é definido por:

$$
\begin{aligned}
B_{2}^{N}: \mathcal{D}^{N}[\mathrm{Top}] & \rightarrow \mathrm{Top}_{*}^{\rightarrow} \\
\left(X_{c}, X_{o}\right) & \mapsto B\left(\Sigma_{2}^{N}, D^{N},\left(X_{c}, X_{o}\right)\right)
\end{aligned}
$$

O seguinte teorema é o resultado central do princípio de reconhecimento de espaços de laços.

Teorema 5.3.2. Seja $\pi_{2}^{N} \in 2 O p(T O p)\left(\mathcal{D}^{N}, \mathcal{S} C^{N}\right)$ uma equivalência fraca entre 2-operads $2 \mathbb{S}$ livres e $\left(\left(X_{c}, X_{o}\right), \xi\right) \in \mathcal{D}^{N}[\mathrm{Top}] \operatorname{com}\left(X_{c}, X_{o}\right) \in$ Top $_{* \text { Cof }}^{2}$ Considere o seguinte diagrama de $\mathcal{D}^{N}$ aplicações:

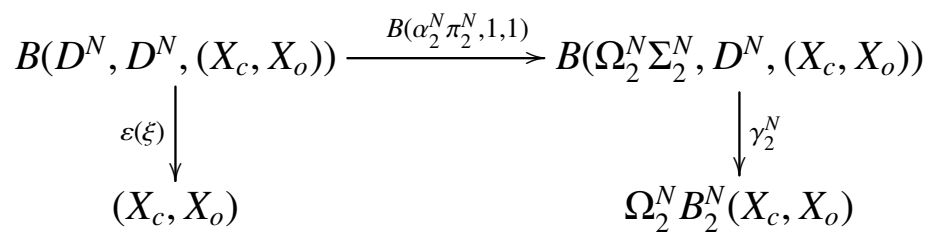

i) $\varepsilon(\xi)$ é um retrato por deformação forte com inverso à direita $\tau\left(\eta^{D^{N}}\right)$;

ii) $B\left(\alpha_{2}^{N} \pi_{2}^{N}, 1,1\right)$ é uma equivalência fraca se $X_{c}$ e $X_{o}$ são conexos e um completamento de grupo se $2<N<\infty$;

iii) $\gamma_{2}^{N}$ é uma equivalência fraca;

iv) A composição $\gamma_{2}^{N} B\left(\alpha_{2}^{N} \pi, 1,1\right) \tau\left(\eta^{\mathcal{D}^{N}}\right)$ coincide com $\Omega_{2}^{N}\left(\tau\left(1_{\Sigma_{2}^{N}}\right)\right) \eta_{2}^{N}$, e é uma equivalência fraca se $X_{c}$ e $X_{o}$ são conexos ou se $2<N<\infty$ e $X_{c}$ e $X_{o}$ são grouplike;

v) $B\left(\Sigma_{2}^{N}, D^{N},\left(X_{c}, X_{o}\right)\right)$ é $m+N$-conexo se $X_{c}$ e $X_{o}$ são m-conexos.

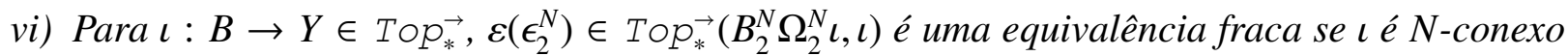

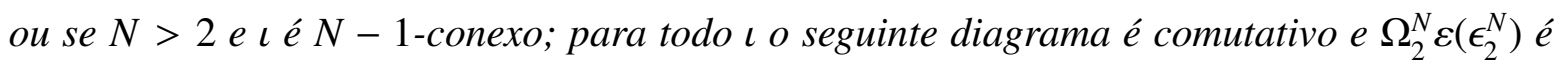


uma retração com inversa à direita $\Omega_{2}^{N}\left(\tau\left(1_{\Sigma_{2}^{N} \Omega_{2}^{N}}\right)\right) \eta_{2 \Omega_{2}^{N}}^{N}$,

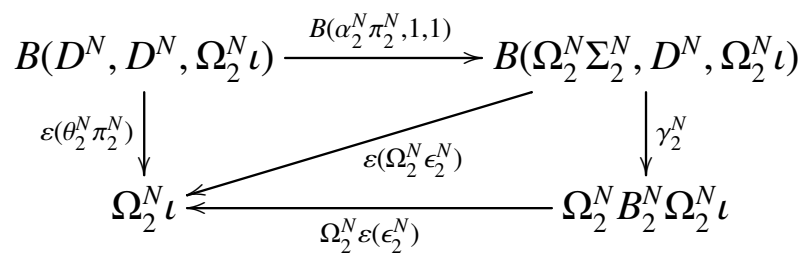

vii) Para $\left(Y_{c}, Y_{o}\right) \in T o p_{*}^{2}, \varepsilon\left(\epsilon_{2 \Sigma_{2}^{N}}^{N} \Sigma_{2}^{N}\left(\alpha_{2}^{N} \pi_{2}^{N}\right)\right) \in \operatorname{Top}_{*}^{2}\left(B_{2}^{N} D^{N}\left(Y_{c}, Y_{o}\right), \Sigma_{2}^{N}\left(Y_{c}, Y_{o}\right)\right)$ é um retrato por deformação forte com inversa à direita $\tau\left(\Sigma_{2}^{N} \eta^{D^{N}}\right)$.

Demonstração: $\varepsilon(\xi)$ e $B\left(\alpha_{2}^{N} \pi_{2}^{N}, 1,1\right)$ são realizações de $\mathcal{D}^{N}$-aplicações simpliciais, logo pela proposição 5.2.2 são $\mathcal{D}^{N}$-aplicações. $\gamma_{2}^{N}$ é uma $\mathcal{D}^{N}$-aplicação por 5.2.5. $i$ ) e vii) valem antes da realização por [Ma72, 9.10, 9.11], logo valem depois da realização por [Ma72, 11.10]. ii) vale antes da realização por 4.3.5 e 5.1.11, e portanto vale depois da realização pelo argumento em [Ma74, T.2.3.ii)]. iii) segue de 5.2.4. $i$ v) segue de i), ii) e iii). v) segue de [Ma72, 11.12] e [Ma74, A.5]. O triangulo de cima de vi) comuta pela naturalidade de $\varepsilon$, e o de baixo por [Ma72, 9.11]. O fato que $\varepsilon\left(\epsilon_{2}^{N}\right)$ é uma equivalência fraca se as condições de conectividade são satisfeitas segue da comutatividade do diagrama e dos ítens anteriores.

O seguinte corolário nos diz que o desenlaçamento de $\mathcal{D}^{N}$-álgebras que são ou conexas ou grouplike se $N>2$ é único a menos de equivalência fraca entre espaços relativos $N$-conexos ou $N-1$-conexos respectivamente.

Corolário 5.3.3. Sob as hipóteses do teorema, considere um span de $\mathcal{D}^{N}$-equivalências fracas:

$$
\left(X_{c}, X_{o}\right) \stackrel{f}{\stackrel{\sim}{\sim}}\left(X_{c}^{\prime}, X_{o}^{\prime}\right) \stackrel{g}{\sim} \Omega_{2}^{N} \iota
$$

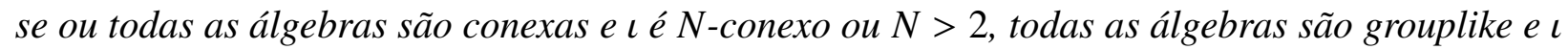
é N-1-conexo, então o diagrama

$$
B_{2}^{N}\left(X_{c}, X_{o}\right) \stackrel{B(1,1, f)}{\sim} B_{2}^{N}\left(X_{c}^{\prime}, X_{o}^{\prime}\right) \stackrel{\varepsilon\left(\epsilon_{2}^{N}\right) B(1,1, g)}{\sim} \iota
$$

nos dá uma equivalência fraca entre ı e $B_{2}^{N}\left(X_{c}, X_{o}\right)$.

Demonstração: $\varepsilon\left(\epsilon_{2}^{N}\right)$ é uma equivalência fraca pelo teorema 5.3.2.vi), e $B(1,1, f)$ e $B(1,1, g)$ são equivalências fracas antes da realização pelo teorema de aproximação relativo, e também depois da realização por [Ma72, 11.13].

Usando a linguagem de quasi-adjunções de Quillen fracas estabelecida na sessão 1.3 podemos mostrar que os funtores do teorema do reconhecimento relativo é compatível com as estruturas modelo de espaços relativos $N-1$-conexos da sessão 3.3 e a estrutura modelo de $\overline{S C^{N}}$-álgebras da sessão 4.3, de forma que eles induzem uma adjunção das categorias homotópicas. 
Teorema 5.3.4. Seja $\pi_{2}^{N} \in 2 O p(T O p)\left(\overline{\mathcal{S} C^{N}}, \mathcal{S} C^{N}\right)$ uma resolução cofibrante. Então

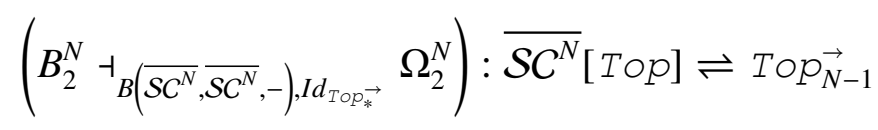

é uma quasi-adjunção de Quillen fraca, e portanto induz uma adjunção das categorias homotópicas:

$$
\left(\mathbb{L} B_{2}^{N} \dashv \mathbb{R} \Omega_{2}^{N}\right): \mathcal{H} o \overline{\mathcal{S C}}^{N}[\mathrm{Top}] \rightleftharpoons \mathcal{H} \text { To } \mathrm{Top}_{N-1}
$$

Demonstração: Provamos que os funtores do teorema, equipados com as transformações na-

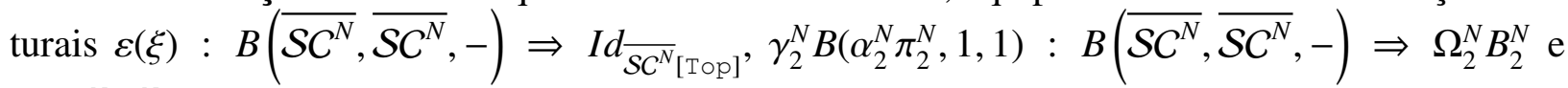
$\varepsilon: B_{2}^{N} \Omega_{2}^{N} \Rightarrow I d_{\mathrm{Top}_{N-1}}$ satisfazem as condições da definição 1.3.8.

Temos que $i$ ) e $i$ ) são satisfeitas pois $B_{2}^{N}$ preserva objetos cofibrantes e equivalências fracas entre eles pelo lema 4.3.4. Temos que $\Omega_{2}^{N}$ trivialmente preserva objetos fibrantes e que preserva equivalências fracas pelas construções das estruturas modelo, logo iii)e $i v$ ) são satisfeitos. Também pelo lema 4.3.4 temos que $B\left(\overline{S C^{N}}, \overline{S C^{N}},-\right)$ preserva objetos cofibrantes e ele trivialmente preserva objetos fibrantes, $\log 0 v$ ) é satisfeito. vi) é trivial pois estamos considerando o funtor identidade. vii) é o teorema 5.3.2.i e viii) é trivial pois a transformação natural considerada é a identidade. $i x$ ) segue do teorema 5.3.2.iv e $x$ ) segue do teorema 5.3.2.vi.

A imagem do funtor $\Omega_{2}^{N}$ está contida na subcategoria de $\overline{\mathcal{S C}}$-álgebras grouplike. Mostramos agora que podemos definir uma localização de Bousfield que captura a subcategoria homotópica reflexiva dessas álgebras.

Teorema 5.3.5. Seja $N>$ 2. O endofuntor $\Omega_{2}^{N} B_{2}^{N}$ Cof induz uma localização de Bousfield à esquerda em $\overline{\mathcal{S} C^{N}}$ [Top] na qual as $\Omega_{2}^{N} B_{2}^{N}$ Cof-equivalências fracas são as $\overline{\mathcal{S C}}$-aplicações $\left(f_{c}, f_{o}\right) \in$ $\overline{S C^{N}}\left(\left(X_{c}, X_{o}\right),\left(X_{c}^{\prime}, X_{o}^{\prime}\right)\right)$ tais que

$$
\begin{gathered}
\bar{f}_{c *} \in k-A \operatorname{lgGr}\left(H_{\bullet}\left(X_{c}, k\right)\left[\pi_{0} X_{c}^{-1}\right], H_{\bullet}\left(X_{c}^{\prime}, k\right)\left[\pi_{0} X_{c}^{\prime-1}\right]\right) \\
\bar{f}_{o *} \in k-A \operatorname{lgGr}\left(H_{\bullet}\left(X_{o}, k\right)\left[\pi_{0} X_{o}^{-1}\right], H_{\bullet}\left(X_{o}^{\prime}, k\right)\left[\pi_{0} X_{o}^{\prime-1}\right]\right)
\end{gathered}
$$

são isomorfismos para todo anel comutativo $k$ e os objetos $\Omega_{2}^{N} B_{2}^{N}$ Cof-fibrantes são as $\overline{\mathcal{S C}}{ }^{N}$. álgebras grouplike.

Demonstração: Primeiro note que para $\left(f_{c}, f_{o}\right) \in \overline{\mathcal{S C}}\left(\left(X_{c}, X_{o}\right),\left(X_{c}^{\prime}, X_{o}^{\prime}\right)\right)$ temos o seguinte 
$\overline{\text { diagrama em } \overline{S C^{N}}}$ [Top]:

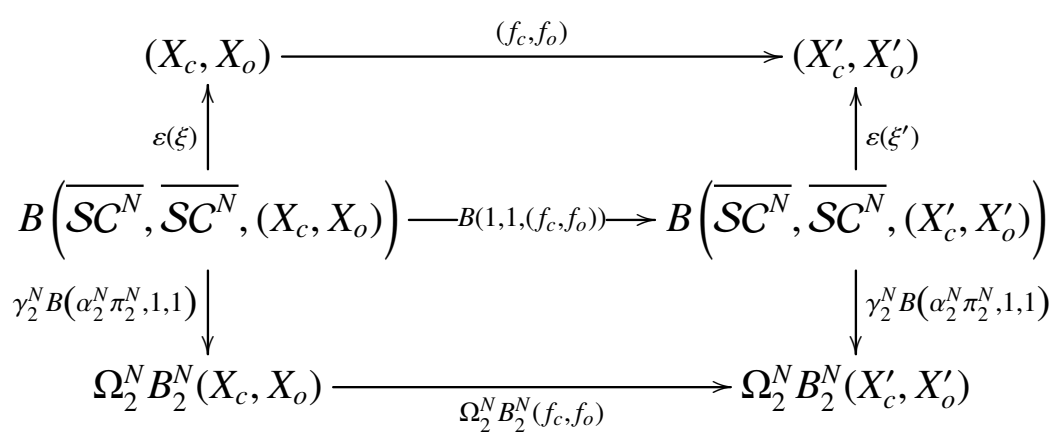

Se $\left(X_{c}, X_{o}\right),\left(X_{c}^{\prime}, X_{o}^{\prime}\right) \in \mathrm{Top}_{* \text { Cof }}^{2}$ o teorema 5.3.2 e o teorema dual de Whitehead [Ma83] nos dão que $\Omega_{2}^{N} B_{2}^{N}\left(f_{c}, f_{o}\right)$ é uma equivalência fraca se e somente se $\left(f_{c}, f_{o}\right)$ satisfaz as condições no enunciado. $\mathrm{O}$ caso geral segue do fato que toda $\overline{S C^{N}}$-álgebra é fracamente equivalente à uma cofibrante.

Provamos que $\Omega_{2}^{N} B_{2}^{N}$ Cof equipado com o endofuntor $B\left(\overline{\mathcal{S C}} C^{N}, \overline{\mathcal{S C}}{ }^{N}\right.$, Cof- $)$ e as transformações

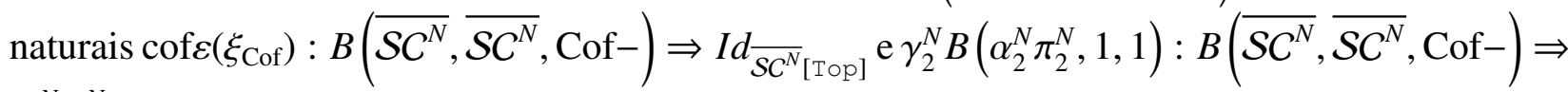
$\Omega_{2}^{N} B_{2}^{N}$ Cof satisfazem as condições da definição 1.8.3.

Que vale $i$ ) segue do teorema 5.3.2.i, que cof é uma fibração trivial e da propriedade doisde-três. Que vale ii) segue da primeira parte da demonstração do teorema anterior. A condição iii) segue da primeira parte dessa demonstração e do teorema 5.3.2.ii. A condição $i v$ ) vale pelo fato que pullbacks de fibrações são fibrações, pela sequência exata longa de fibrações de Serre e pelo lema dos 5. A condição $v)$ segue do fato que as imagens de $B\left(\overline{\mathcal{S C} C^{N}}, \overline{\mathcal{S C} C^{N}}\right.$, Cof- $)$ são $\overline{\mathcal{S} C^{N}}$ álgebras cofibrantes e pelo fato que pushouts de equivalências fracas por cofibrações com domínio cofibrante em uma categoria de álgebras sobre um operad cofibrante em uma categoria própria à esquerda é uma equivalência fraca [Sp01, S.4 T.4].

Que os objetos $\Omega_{2}^{N} B_{2}^{N}$ Cof-fibrantes são as $\overline{S C^{N}}$-álgebras grouplike segue da proposição 1.8.8.

Denotamos a localização de Bousfield à esquerda induzida pela quasi-monada idempotente de Quillen do teorema por $\overline{\mathcal{S C}}^{N}$ [Top $]_{\text {Grp. }}$. Com essa nova estrutura modelo obtemos uma equivalência de categorias homotopicas.

Teorema 5.3.6. Seja $N>$ 2. Os funtores $\Omega_{2}^{N}$ e $B_{2}^{N}$ induzem uma equivalência de categorias homotópicas:

$$
\left(\mathbb{L} B_{2}^{N} \dashv \mathbb{R} \Omega_{2}^{N}\right): \mathcal{H} o \overline{S C}^{N}[T o p]_{G r p} \rightleftharpoons \mathcal{H o T o p} \overrightarrow{N-1}
$$

Demonstração: Pelo teorema 5.3.2 as condições do teorema 1.3.10 são satisfeitas

Pelo teorema 4.4.4 podemos usar a resolução cofibrante $\mathfrak{W}_{I} \mathcal{F} \mathcal{M}_{2}^{N}$ de $\mathcal{S} C^{N}$ para obter uma categoria de álgebras explícita.

Para $N \leq 2$ podemos adaptar esses argumentos para provar uma equivalência entre a categoria homotópica de $\overline{S C^{N}}$-álgebras conexas e a categoria de espaços relativos $N$-conexos. O caso geral 
para $N=1$ é uma consequência dos resultados em [HLS16]. A dificuldade de fazer a generalização para $N=2$ vem do fato que as $C^{1}$-álgebras não são no geral admissíveis, então $\alpha_{2}^{2}$ pode não ser uma equivalência fraca em álgebras grouplike, e os resultados em [HLS16] usam o fato que $S C^{1}$ é fracamente equivalente ao 2 -operad $2 \mathbb{S}$-livre discreto de ações de monóides topológicos em espaços $A s s^{\text {act }}$, o que não é verdade para $S C^{2}$. O caso geral para $N=2$ continua aberto.

\subsection{Princípio de reconhecimento de laços relativos infinitos}

Considere o $2 E_{\infty}$-operad $\mathfrak{W}_{I} \mathcal{F} \mathcal{M}_{2}^{\infty}$ e um outro $2 E_{\infty}$-operad $\mathcal{E}_{2}^{\infty}$ qualquer. Temos um 2-operad produto $\mathcal{E}_{2}^{\infty} \times \mathfrak{W}_{I} \mathcal{F} \mathcal{M}_{2}^{\infty}$ que é definido como o produto em cada índice que também é um $2 E_{\infty}$ operad. Temos então que pelo teorema de Whitehead 1.2.12 existe uma inversa homotópica $\tilde{p}_{1}$ da projeção $p_{1} \in 2 O p(\mathrm{Top})\left(\mathcal{E}_{2}^{\infty} \times \mathfrak{W}_{I} \mathcal{F} \mathcal{M}_{2}^{\infty}, \mathcal{E}_{2}^{\infty}\right)$. Definimos então $\pi^{\prime}:=p_{2} \tilde{p}_{1}$, com $p_{2} \in 2 O p(\mathrm{Top})$ $\left(\mathcal{E}_{2}^{\infty} \times \mathfrak{W}_{I} \mathcal{F} \mathcal{M}_{2}^{\infty}, \mathfrak{W}_{I} \mathcal{F} \mathcal{M}_{2}^{\infty}\right)$ a outra projeção. Pelo teorema de Whitehead $\pi^{\prime}$ tem uma inversa homotópica que nos dá que toda $\mathcal{E}_{2}^{\infty}$-álgebra é uma $\mathfrak{B}_{I} \mathcal{F} \mathcal{M}_{2}^{\infty}$-álgebra, logo como $\mathfrak{B}_{I} \mathcal{F} \mathcal{M}_{2}^{\infty}$ é definido como um colimite das inclusões $\sigma^{N} \in 2 O p(\mathrm{Top})\left(\mathfrak{W}_{I} \mathcal{F} \mathcal{M}_{2}^{N}, \mathfrak{M}_{I} \mathcal{F} \mathcal{M}_{2}^{N+1}\right)$ elas também são $\mathfrak{W}_{I} \mathcal{F} \mathcal{M}_{2}^{N}$-álgebras para todo $N$. Essas inclusões e os homeomorfismos $\mathbb{S}^{N} \wedge \mathbb{S}^{1} \rightarrow \mathbb{S}^{N+1}$ induzem uma aplicação

$$
\Sigma^{1 \rightarrow} B\left(\Sigma_{2}^{N}, W_{I} F M_{2}^{N},\left(X_{c}, X_{o}\right)\right) \rightarrow B\left(\Sigma_{2}^{N+1}, W_{I} F M_{2}^{N+1},\left(X_{c}, X_{o}\right)\right)
$$

que dá a essa coleção de espaços uma estrutura de espectro relativo, com $W_{I} F M_{2}^{N}$ a monada associada ao 2-operad $\mathfrak{W}_{I} \mathcal{F} \mathcal{M}_{2}^{N}$. A definição a seguir nos dá um funtor de desenlaçamento infinito.

Definição 5.4.1. O funtor de desenlaçamento infinito de $\mathcal{E}_{2}^{\infty}$-álgebras para um $2 E_{\infty}$-operad $\mathcal{E}_{2}^{\infty}$ é

$$
\begin{aligned}
B_{2}^{\infty}: \mathcal{E}_{2}^{\infty}[\mathrm{Top}] & \rightarrow \mathrm{Sp}^{\rightarrow} \\
\left(X_{c}, X_{o}\right) & \mapsto B\left(\Sigma_{2}^{\bullet}, W_{I} F M_{2}^{\bullet},\left(X_{c}, X_{o}\right)\right)
\end{aligned}
$$

Lembre que pelo teorema 4.4.4 temos uma resoluções cofibrantes $\mathfrak{v} \in 2 O p(\mathrm{Top})\left(\mathfrak{B}_{1} \mathcal{F} \mathcal{M}_{2}^{\infty}, \mathcal{S} C^{\infty}\right)$. Os resultados da sessão anterior então induzem os seguintes resultados.

Teorema 5.4.2. Seja $\left(X_{c}, X_{o}\right) \in \mathcal{E}_{2}^{\infty}[\mathrm{Top}] \operatorname{com}\left(X_{c}, X_{o}\right) \in T o p_{* \text { Cof }}^{2}$ Considere o seguinte diagrama de $\mathcal{E}_{2}^{\infty}$-aplicações:

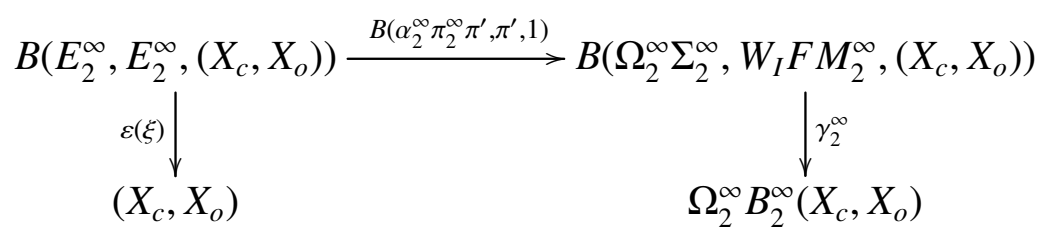

i) $\varepsilon(\xi)$ é um retrato por deformação forte com inverso à direita $\tau\left(\eta^{\mathcal{E}_{2}^{\infty}}\right)$;

ii) $B\left(\alpha_{2}^{\infty} \pi_{2}^{\infty} \pi^{\prime}, \pi_{2}^{\infty} \pi^{\prime}, 1\right)$ é um completamento de grupo. 
iii) $\gamma_{2}^{\infty}$ é uma equivalência fraca;

iv) A composição $\gamma_{2}^{\infty} B\left(\alpha_{2}^{\infty} \pi_{2}^{\infty} \pi^{\prime}, \pi_{2}^{\infty} \pi^{\prime}, 1\right) \tau\left(\eta^{\mathcal{E}_{2}^{N}}\right)$ coincide com $\Omega_{2}^{\infty}\left(\tau\left(1_{\Sigma_{2}^{\infty}}\right)\right) \eta_{2}^{\infty}$, e é uma equivalência fraca se $X_{c}$ e $X_{o}$ são grouplike;

v) $B_{2}^{\infty}\left(X_{c}, X_{o}\right)$ é conectivo;

vi) Para $\left(\iota_{\bullet}: B_{\bullet} \rightarrow Y_{\bullet}\right) \in S p^{\nearrow}, \varepsilon\left(\epsilon_{2}^{\infty}\right): B_{2}^{\infty} \Omega_{2}^{\infty} \iota_{\bullet} \rightarrow \iota_{\bullet}$ é uma equivalência fraca se $\iota_{\bullet}$ é connectivo; para todo $\iota_{\text {. }}$ o seguinte diagrama é comutativo e $\Omega_{2}^{\infty} \varepsilon\left(\epsilon_{2}^{\infty}\right)$ é uma retração com inversa à direita $\Omega_{2}^{\infty}\left(\tau\left(1_{\Sigma_{2}^{\infty} \Omega_{2}^{\infty}}\right)\right) \eta_{2 \Omega_{2}^{\infty}}^{\infty}$

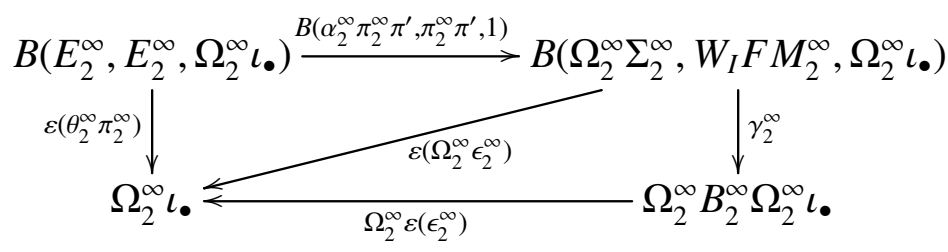

vii) Para $\left(Y_{c}, Y_{o}\right) \in T o p_{*}^{2}, \varepsilon\left(\epsilon_{2 \Sigma_{2}^{\infty}}^{\infty} \Sigma_{2}^{\infty}\left(\alpha_{2}^{\infty} \pi_{2}^{\infty}\right)\right) \in \operatorname{Top}_{*}^{2}\left(B_{2}^{\infty} E_{2}^{\infty}\left(Y_{c}, Y_{o}\right), \Sigma_{2}^{\infty}\left(Y_{c}, Y_{o}\right)\right)$ é um retrato por deformação forte com inversa à direita $\tau\left(\Sigma_{2}^{\infty} \eta^{\mathcal{E}_{2}^{\infty}}\right)$.

Demonstração: A demonstração é análoga à demonstração de 5.3.2 com a ressalva que precisamos usar que há uma equivalência fraca entre $\Omega_{2}^{\infty} B\left(\Sigma_{2}^{\infty}, W_{I} F M_{2}^{\infty},\left(X_{c}, X_{o}\right)\right)$ e $\Omega_{2}^{\infty} B_{2}^{\infty}\left(X_{c}, X_{o}\right)$.

Temos que o desenlaçamento infinito de uma $\mathcal{E}_{2}^{\infty}$-álgebra grouplike é único a menos de equivalência fraca.

Corolário 5.4.3. Sob as hipóteses do teorema, considere o seguinte diagrama de $\mathcal{E}_{2}^{\infty}$-álgebras:

$$
\left(X_{c}, X_{o}\right) \stackrel{f}{\longleftarrow}\left(X_{c}^{\prime}, X_{o}^{\prime}\right) \stackrel{g}{\longrightarrow} \Omega_{2}^{\infty} \iota \text {. }
$$

se $f$ e g são equivalências fracas de $\mathcal{E}_{2}^{\infty}$-álgebras grouplike e $\iota_{\bullet}$ é conectivo, então o diagrama de espectros relativos

$$
B_{2}^{\infty}\left(X_{c}, X_{o}\right) \stackrel{B(1,1, f)}{\stackrel{\infty}{\infty}}\left(X_{c}^{\prime}, X_{o}^{\prime}\right) \stackrel{\varepsilon\left(\epsilon_{2}^{N}\right) B(1,1, g)}{\longrightarrow} \iota \bullet
$$

nos dá uma equivalência fraca entre ı e $B_{2}^{N}\left(X_{c}, X_{o}\right)$.

Novamente temos que $\Omega_{2}^{\infty}$ e $B_{2}^{\infty}$ são compatíveis com as estruturas modelo e induzem uma adjunção das categorias homotópicas.

Teorema 5.4.4. Seja $\mathcal{E}_{2}^{\infty}$ um $2 E_{\infty}$-operad. Então

$$
\left(B_{2}^{\infty} \dashv_{B\left(E_{2}^{\infty}, E_{2}^{\infty},-\right), I d_{S p} \backslash} \Omega_{2}^{\infty}\right): \mathcal{E}_{2}^{\infty}[\mathrm{TOP}] \rightleftharpoons S p^{\nearrow}
$$

é uma quasi-adjunção de Quillen fraca, e portanto induz uma adjunção das categorias homotópicas:

$$
\left(\mathbb{L} B_{2}^{\infty} \dashv \mathbb{R} \Omega_{2}^{\infty}\right): \mathcal{H} o \mathcal{E}_{2}^{\infty}[T o p] \rightleftharpoons \mathcal{H} o S p^{\nearrow}
$$


Demonstração: O argumento é o mesmo que o do teorema 5.3.4.

Assim como no caso finito também obtemos uma localização de Bousfield à esquerda que nos dá a subcategoria homotópica de $\mathcal{E}_{2}^{\infty}$-álgebras grouplike.

Teorema 5.4.5. O endofuntor $\Omega_{2}^{\infty}$ Fib $B_{2}^{\infty}$ Cof induz uma localização de Bousfield à esquerda em $\mathcal{E}_{2}^{\infty}$ [Top] na qual as $\Omega_{2}^{\infty}$ FibB $B_{2}^{\infty}$ Cof-equivalências fracas são as $\mathcal{E}_{2}^{\infty}$-aplicações $\left(f_{c}, f_{o}\right) \in \mathcal{E}_{2}^{\infty}\left(\left(X_{c}, X_{o}\right)\right.$, $\left.\left(X_{c}^{\prime}, X_{o}^{\prime}\right)\right)$ tais que

$$
\begin{gathered}
\left.\bar{f}_{c *} \in k-A\right] g G r\left(H_{\bullet}\left(X_{c}, k\right)\left[\pi_{0} X_{c}^{-1}\right], H_{\bullet}\left(X_{c}^{\prime}, k\right)\left[\pi_{0} X_{c}^{\prime-1}\right]\right) \\
\bar{f}_{o *} \in k-A \operatorname{gGr}\left(H_{\bullet}\left(X_{o}, k\right)\left[\pi_{0} X_{o}^{-1}\right], H_{\bullet}\left(X_{o}^{\prime}, k\right)\left[\pi_{0} X_{o}^{\prime-1}\right]\right)
\end{gathered}
$$

são isomorfismos para todo anel comutativo $k$ e os objetos $\Omega_{2}^{\infty}$ FibB $B_{2}^{\infty}$ Cof-fibrantes são as $\mathcal{E}_{2}^{\infty}$ álgebras grouplike.

Demonstração: O argumento é o mesmo que o do teorema 5.3.5.

Denotamos a localização de Bousfield à esquerda induzida pela quasi-monada idempotente de Quillen do teorema por $\mathcal{E}_{2}^{\infty}[\mathrm{T} \circ \mathrm{p}]_{\text {Grp }}$. No caso infinito, a existência de sequências exatas longas de grupos de homotopia estáveis de cofibrações nos permitem definir uma localização de Bousfield à direita da categoria modelo estável de espectros relativos que nos dão a subcategoria homotópica correflexiva de espectros relativos conectivos.

Teorema 5.4.6. O endofuntor $B_{2}^{\infty} \operatorname{Cof} \Omega_{2}^{\infty} \mathrm{Fib}$ induz uma localização de Bousfield à direita em $\mathrm{Sp}^{\nearrow}$ na qual as $B_{2}^{\infty} \mathrm{Cof} \Omega_{2}^{\infty}$ Fib-equivalências fracas são as aplicações de espectros relativos $\left(k_{\bullet}, l_{\bullet}\right) \in$ $\operatorname{Sp}^{\nearrow}\left(\iota_{\bullet}: B_{\bullet} \rightarrow Y_{\bullet+1}, \iota_{\bullet}^{\prime}: B_{\bullet}^{\prime} \rightarrow Y_{\bullet+1}^{\prime}\right)$ tais que

$$
\begin{gathered}
k_{\bullet *} \in \operatorname{Grp}\left(\pi_{q}^{S} B_{\bullet}, \pi_{q}^{S} B_{\bullet}^{\prime}\right) \\
\left(k_{\bullet},\left[1_{I}, l_{\bullet+1}\right]_{*}\right)_{*} \in \operatorname{Grp}\left(\pi_{q}^{S}\left(B_{\bullet} \times_{Y_{\bullet+1}}\left[I, Y_{\bullet+1}\right]_{*}\right), \pi_{q}^{S}\left(B_{\bullet} \times_{Y_{\bullet+1}}\left[I, Y_{\bullet+1}\right]_{*}\right)\right)
\end{gathered}
$$

são isomorfismos para todo $q \geq 0$ e os objetos $B_{2}^{\infty} \mathrm{Cof} \Omega_{2}^{\infty}$ Fib-cofibrantes são os espectros relativos conectivos.

Demonstração: Primeiro note que para $\left(k_{\bullet}, l_{\bullet}\right) \in \mathrm{Sp}^{\nearrow}\left(\iota, \iota^{\prime}\right)$ temos o seguinte diagrama em $\mathrm{Sp}^{\nearrow}$ :

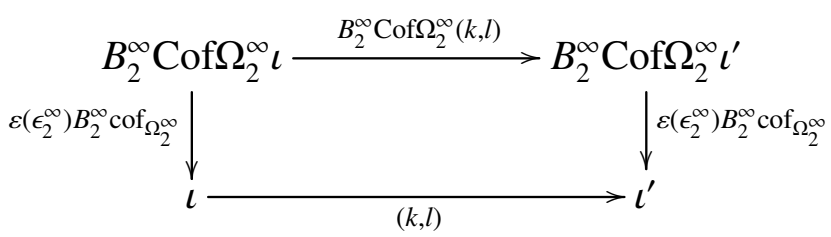

Pela mesma computação do final da proposição 3.2.6 e o lema dos 5 temos que o teorema 5.4.2.vi) nos dá que $(k, l)$ é uma $B_{2}^{\infty} \operatorname{Cof} \Omega_{2}^{\infty}$ Fib-equivalência fraca se e somente se satisfaz as condições do enunciado. 
Provamos que $B_{2}^{\infty} \mathrm{Cof} \Omega_{2}^{\infty} \mathrm{Fib}$ equipado com o endofuntor Fib e as transformações naturais $\varepsilon\left(\epsilon_{2 \mathrm{Fib}}^{\infty}\right) B_{2}^{\infty} \operatorname{cof}_{\Omega_{2}^{\infty} \mathrm{Fib}}: B_{2}^{\infty} \mathrm{Cof} \Omega_{2}^{\infty} \mathrm{Fib} \Rightarrow$ Fib e fib :Id $d_{\mathrm{Sp}} \Rightarrow$ Fib satisfazem as condições de uma quasi-comonada idempotente duais às condições na definição 1.8.3.

Que vale $i$ ) segue de fib ser uma cofibração trivial. Que vale $i i)$ segue por $B_{2}^{\infty}$ preservar equivalências fracas enter objetos cofibrantes pelo lema 4.3.4 e $\Omega_{2}^{\infty}$ preserva equivalências fracas entre objetos fibrantes pela proposição 3.3.14. A condição iii) segue da primeira parte dessa demonstração e do teorema 5.4.2.vi). A condição $i v$ ) vale pelo fato que pushouts de cofibrações são cofibrações, pela sequência exata longa de grupos de homotopia estáveis da proposição 3.2.7 e pelo lema dos 5 . A condição $v$ ) segue do fato que $\mathrm{Sp}^{\nearrow}$ é próprio à direita.

Que os objetos $B_{2}^{\infty} \operatorname{Cof} \Omega_{2}^{\infty}$ Fib-cofibrantes são os espectros relativos conectivos segue do dual da proposição 1.8 .8 .

Denotamos a localização de Bousfield à esquerda induzida pela quasi-comonada idempotente de Quillen do teorema por $\mathrm{Sp}_{\mathrm{Con}}^{\nearrow}$. Obtemos assim uma equivalência das subcategorias homotópicas.

Teorema 5.4.7. Os funtores $\Omega_{2}^{\infty}$ e $B_{2}^{\infty}$ induzem uma equivalência de categorias homotópicas:

$$
\left(\mathbb{L} B_{2}^{\infty} \dashv \mathbb{R} \Omega_{2}^{\infty}\right): \mathcal{H} o \mathcal{E}_{2}^{\infty}[T o p]_{G r p} \rightleftharpoons \mathcal{H} o S p_{C o n}^{\nearrow}
$$

Demonstração: Pelos últimos teoremas as condições do teorema 1.9.2 são satisfeitas. 



\section{Apêndice A}

\section{(Não-)formalidade do 2-operad de queijos suíços de Voronov em dimensão 2}

Seja $k$ um corpo de característica 0 . Os funtores $C \cdot(-, k), H_{\bullet}(-, k):$ Top $\rightarrow \mathrm{Ch}_{\geq 0}(k-m o d)$ de complexos de cadeia com coeficientes em $k$ e de homologia com coeficientes em $k$ respectivamente são monoidais simétricos, e portanto preservam a estrutura de operads. Dizemos que um operad topológico $\mathcal{P}$ é formal se existe um span de equivalências fracas de operads de complexos de cadeia em $k$ entre $C^{\bullet}(\mathcal{P}, k)$ e $H^{\bullet}(\mathcal{P}, k)$. O teorema de quantização por deformação de Kontsevich [Ko99] pode ser derivado do fato que o operad dos pequenos $N$-discos (ou equivalentemente dos pequenos $N$-cubos) é formal [Ta03]. Uma demonstração detalhada da formalidade do operad dos pequenos discos se encontra em [LV14].

Em [Li15] Livernet provou que os 2-operads reduzidos dos queijos-suíços $\mathcal{S} C^{N}$ como definida nessa tese e usado na teoria de deformação do Kontsevich não é formal usando uma versão para operads de produtos de Massey. A definição original do Voronov do operad dos queijos suíços assumia que $\mathcal{S} C_{\text {Vor }}^{2}(\underline{n, 0})=\emptyset$ para todo $n \in \mathbb{N}$. Note que em particular esse operad não é reduzido na parte aberta. A demonstração da Livernet usa um elemento de $\mathcal{S} C^{N}(\underline{1,0)}$ nos produtos de Massey, e portanto não é aplicável para a versão do Voronov desse 2-operad. Nesse apêndice mostramos que o argumento da Livernet pode ser adaptado para mostrar que a versão Voronov do 2-operad dos queijos suíços em dimensão 2 também não é formal.

Considere os seguintes elementos dos complexos de cadeia singulares dos queijos suíços:
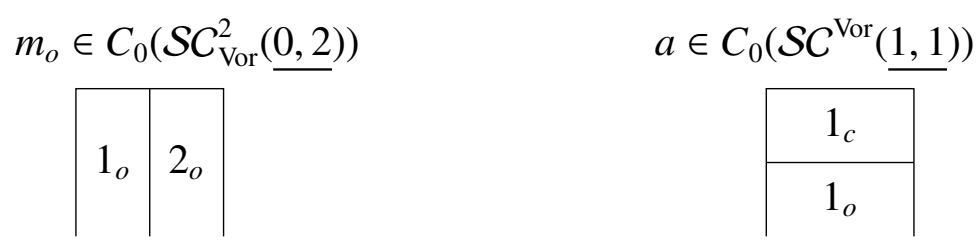

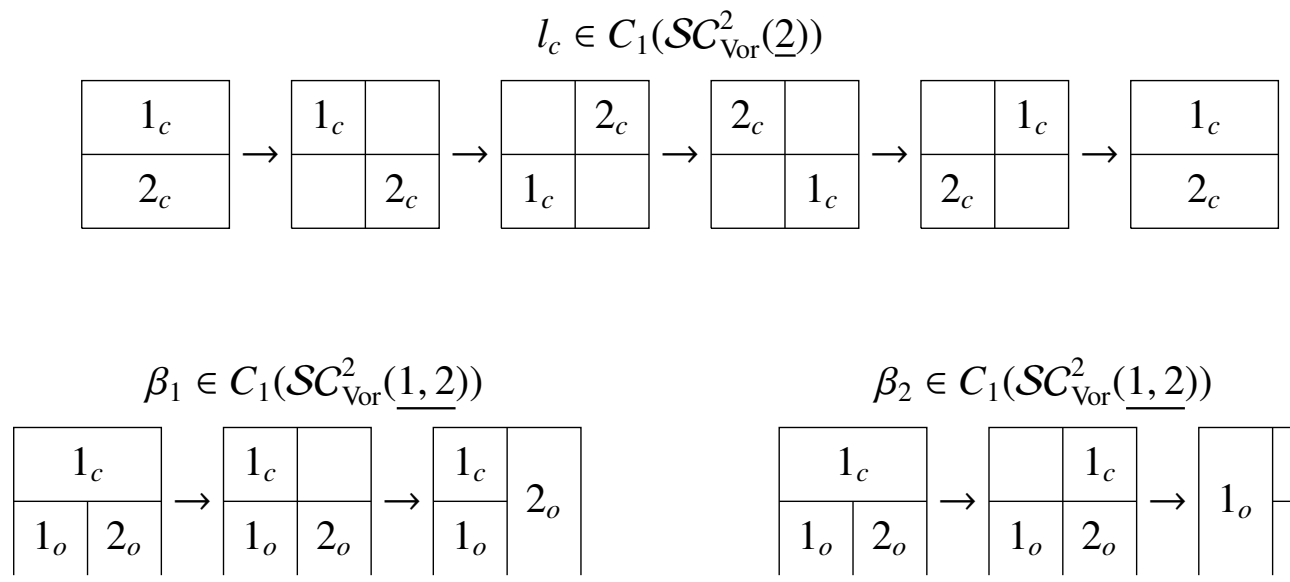

$$
\begin{aligned}
& \beta_{2} \in C_{1}\left(\mathcal{S} C_{\mathrm{Vor}}^{2}(1,2)\right) \\
& \begin{array}{|c|c|c|}
\hline \multicolumn{1}{|c|}{1_{c}} \\
\hline 1_{o} & 2_{o} \\
\hline & \frac{1}{1_{c}} \\
\hline 1_{o} & 2_{o}
\end{array} \rightarrow \begin{array}{|l|l|}
\hline 1_{o} & 1_{c} \\
\cline { 2 - 3 } & 2_{o}
\end{array}
\end{aligned}
$$

Note que $m_{o}$ é um ciclo que gera o $\mathbb{S}_{\underline{2}}$-módulo $H_{0}\left(\mathcal{S C}_{\mathrm{Vor}}^{2}(\underline{0,2)})\right.$ e que $a$ e $l_{c}$ são ciclos que geram respectivamente os espaços vetoriais unidimensionais $H_{0}\left(\mathcal{S} C_{\text {Vor }}^{2}(\underline{1,1})\right)$ e $H_{1}\left(\mathcal{S} C_{\text {Vor }}^{2}(2)\right)$. Além disso temos $\partial \beta_{1}=-a\left(1 ; m_{o}\right)+m_{o}(a, 1)$ e $\partial \beta_{2}=-a\left(1 ; m_{o}\right)+m_{o}(1, a)$.

Considere $\eta \in C_{1}\left(S C_{\text {Vor }}^{2} \underline{(2,2)}\right)$ definido por

$$
\eta:=a\left(1 ; \beta_{2}\right)+\beta_{1}(1 ; 1, a)-\left(\beta_{2}(1 ; a, 1)+a\left(1 ; \beta_{1}\right)\right)((21) ;(12))
$$

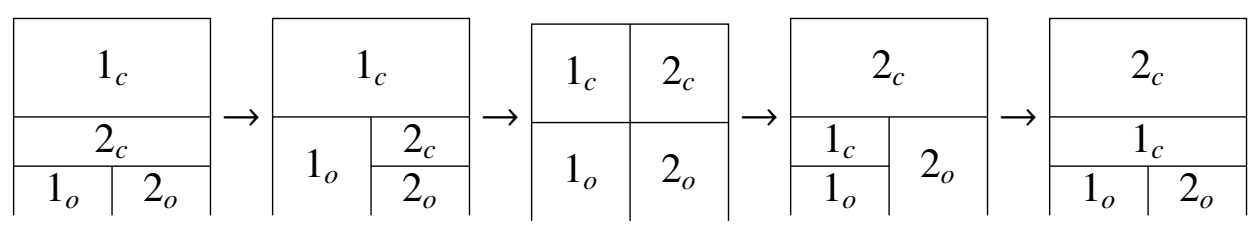

$\operatorname{com} \partial \eta=-a\left(1 ; a\left(1 ; m_{o}\right)\right)+a\left(1 ; a\left(1 ; m_{o}\right)\right)((21) ;(12))$. Temos $[\eta(I d+((21) ;(12)))]=\left[a\left(l_{c} ; m_{o}\right)\right] \mathrm{em}$ $H_{2}\left(S C_{\text {Vor }}^{2} \underline{(2,2)}\right)$ e portanto existe $\gamma \in C_{2}\left(\mathcal{S C} C_{\text {Vor }}^{2} \underline{(2,2)}\right)$ tal que $\partial \gamma=\eta(I d+((21) ;(12)))-a\left(l_{c} ; m_{o}\right)$.

Proposição A.1. Se $m_{o}^{\prime} \in C_{0}\left(\mathcal{S C}_{V o r}^{2}(0,2)\right), a^{\prime} \in C_{0}\left(\mathcal{S} C_{V o r}^{2}(\underline{1,1})\right)$ e $l_{c}^{\prime} \in C_{1}\left(\mathcal{S} C_{V o r}^{2}(2)\right)$ satisfazem

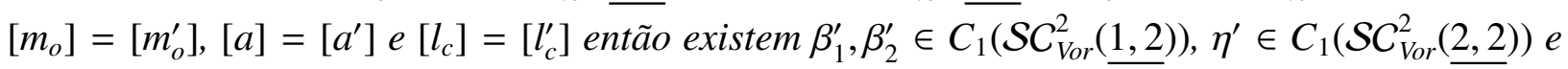
$\gamma^{\prime} \in C_{2}\left(S C_{\text {Vor }}^{2} \underline{(2,2)}\right)$ tais que

$$
\begin{aligned}
& \partial \beta_{1}^{\prime}=-a^{\prime}\left(1 ; m_{o}^{\prime}\right)+m_{o}^{\prime}\left(a^{\prime}, 1\right) \\
& \partial \beta_{2}^{\prime}=-a^{\prime}\left(1 ; m_{o}^{\prime}\right)+m_{o}^{\prime}\left(1, a^{\prime}\right) ; \\
& \partial \eta^{\prime}=-a^{\prime}\left(1 ; a^{\prime}\left(1 ; m_{o}^{\prime}\right)\right)+a^{\prime}\left(1 ; a^{\prime}\left(1 ; m_{o}^{\prime}\right)\right)((21) ;(12)) \\
& \partial \gamma^{\prime}=\eta^{\prime}(I d+((21) ;(12)))-a^{\prime}\left(l_{c}^{\prime} ; m_{o}^{\prime}\right) .
\end{aligned}
$$

Demonstração: Pela hipótese existem $\bar{m}_{o}, \bar{a}$ e $\bar{l}$ tais que $m_{o}^{\prime}=m_{o}+\partial \bar{m}_{o}, a^{\prime}=a+\partial \bar{a}$ e 
$l_{c}^{\prime}=l_{c}+\partial \bar{l}_{c}$. Definimos então

$$
\begin{aligned}
\beta_{1}^{\prime} & :=-a^{\prime}\left(1 ; \bar{m}_{o}\right)-\bar{a}\left(1 ; m_{o}\right)+\beta_{1}+m_{o}(\bar{a}, 1)+\bar{m}_{o}\left(a^{\prime}, 1\right) \\
\beta_{2}^{\prime} & :=-a^{\prime}\left(1 ; \bar{m}_{o}\right)-\bar{a}\left(1 ; m_{o}\right)+\beta_{2}+m_{o}(1, \bar{a})+\bar{m}_{o}\left(1, a^{\prime}\right) \\
\eta^{\prime} & :=a^{\prime}\left(1 ; \beta_{2}^{\prime}\right)+\beta_{1}^{\prime}\left(1 ; 1, a^{\prime}\right)-\left(\beta_{2}^{\prime}\left(1 ; a^{\prime}, 1\right)+a^{\prime}\left(1 ; \beta_{1}^{\prime}\right)\right)((21)
\end{aligned}
$$

que satisfazem as respectivas relações. Como $H_{1}\left(\mathcal{S} C_{\text {Vor }}^{2} \underline{(1,2)}\right)=0$ existem $\bar{\beta}_{1}, \bar{\beta}_{2} \in C_{2}\left(\mathcal{S} C_{\text {Vor }}^{2}(\underline{1,2})\right)$ tais que $\beta_{1}^{\prime}=\beta_{1}+\partial \bar{\beta}_{1}$ e $\beta_{2}^{\prime}=\beta_{2}+\partial \bar{\beta}_{2}$ e definimos

$$
\begin{aligned}
\gamma^{\prime}:= & \gamma+\left(\bar{a}\left(1 ; \beta_{2}\right)+\beta_{1}(1 ; 1, \bar{a})-\left(\beta_{2}(1 ; \bar{a}, 1)+\bar{a}\left(1 ; \beta_{1}\right)\right)((21) ;(12))\right)(I d+((21) ;(12))) \\
& +\left(a^{\prime}\left(1 ; \bar{\beta}_{2}\right)+\bar{\beta}_{1}\left(1 ; 1, a^{\prime}\right)-\left(\bar{\beta}_{2}\left(1 ; a^{\prime}, 1\right)+a^{\prime}\left(1 ; \bar{\beta}_{1}\right)\right)((21) ;(12))\right)(I d+((21) ;(12))) \\
& -\bar{a}\left(l_{c} ; m_{o}\right)-a^{\prime}\left(\bar{l}_{c} ; m_{o}\right)-a^{\prime}\left(l_{c}^{\prime} ; \bar{m}_{o}\right)
\end{aligned}
$$

que satisfaz a respectiva relação.

Teorema A.2. O 2-operad $S C_{V o r}^{2}$ não é formal.

Demonstração: Assuma por contradição que exista um dg-2-operad $Q$ e um span de quasiisomorfismos de operads

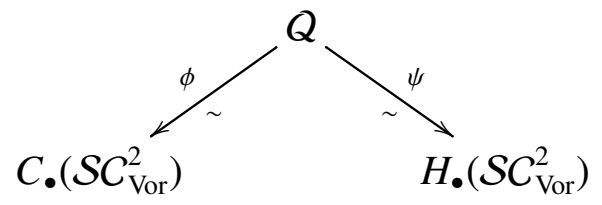

Por $\phi$ ser um quasi-isomorfismo existem ciclos $m_{o}^{Q}, a^{Q}$ e $l_{c}^{Q}$ tais que $\left[\phi\left(x^{Q}\right)\right]=[x]$, para tod $x \in\left\{m_{o}, a, l_{c}\right\}$. Existem $m_{o}^{\prime}$, $a^{\prime}$ e $l_{c}^{\prime}$ como na proposição acima tais que $\phi\left(x^{Q}\right)=x^{\prime}$, para todo $x \in\left\{m_{o}, a, l_{c}\right\}$.

Temos pela existência de $\beta_{1}$ e $\beta_{2}$ que

$$
\begin{aligned}
& H(\phi)\left(-\left[a^{Q}\left(1 ;\left[m_{o}^{Q}\right]\right)\right]+\left[m_{o}^{Q}\left(\left[a^{Q}\right], 1\right)\right]\right)=0 \\
& H(\phi)\left(-\left[a^{Q}\left(1 ;\left[m_{o}^{Q}\right]\right)\right]+\left[m_{o}^{Q}\left(1,\left[a^{Q}\right]\right)\right]\right)=0
\end{aligned}
$$

e por $\phi$ ser um quasi-isomorfismo existem $\beta_{1}^{Q}$ e $\beta_{2}^{Q}$ tais que

$$
\begin{aligned}
& \partial \beta_{1}^{Q}=-a^{Q}\left(1 ; m_{o}^{Q}\right)+m_{o}^{Q}\left(a^{Q}, 1\right) \\
& \partial \beta_{2}^{Q}=-a^{Q}\left(1 ; m_{o}^{Q}\right)+m_{o}^{Q}\left(1, a^{Q}\right)
\end{aligned}
$$

Aplicando $\phi$ e a proposição A.1 obtemos

$$
\begin{aligned}
& \partial \phi\left(\beta_{1}^{Q}\right)=\phi\left(\partial \beta_{1}^{Q}\right)=-a^{\prime}\left(1 ; m_{o}^{\prime}\right)+m_{o}^{\prime}\left(a^{\prime}, 1\right)=\partial \beta_{1}^{\prime} \\
& \partial \phi\left(\beta_{2}^{Q}\right)=\phi\left(\partial \beta_{2}^{Q}\right)=-a^{\prime}\left(1 ; m_{o}^{\prime}\right)+m_{o}^{\prime}\left(1, a^{\prime}\right)=\partial \beta_{2}^{\prime}
\end{aligned}
$$


Definindo

$$
\eta^{Q}:=a^{Q}\left(1 ; \beta_{2}^{Q}\right)+\beta_{1}^{Q}\left(1 ; 1, a^{Q}\right)-\left(\beta_{2}^{Q}\left(1 ; a^{Q}, 1\right)+a^{Q}\left(1 ; \beta_{1}^{Q}\right)\right)((21) ;(12))
$$

obtemos que

$$
\partial\left(\eta^{Q}(I d+((21) ;(12)))\right)=0 \in Q(\underline{2,2})_{0}
$$

O grupo de homologia $H_{1}\left(\mathcal{S C} C^{\text {Vor }}(2,2)\right)$ é gerado por $\left[a\left(l_{c} ; m_{o}\right)\right]=\left[a^{\prime}\left(l_{c}^{\prime} ; m_{o}^{\prime}\right)\right]$, o que implica que $H_{1}(Q(\underline{2,2}))$ é gerado por $\left[a^{Q}\left(l_{c}^{Q} ; m^{Q}\right)\right]$ e que existem $\gamma^{Q} \in Q(\underline{2,2})_{2}$ e $\lambda \in k$ tais que

$$
\eta^{Q}(I d+((21) ;(12)))=\lambda\left(a^{Q}\left(l_{c}^{Q} ; m_{o}^{Q}\right)\right)+\partial \gamma^{Q}
$$

Como $H_{1}\left(\mathcal{S} C_{\text {Vor }}^{2} \underline{(1,2)}\right)=0$ existem $x_{1}, x_{2} \in C_{2}\left(\mathcal{S} C_{\text {Vor }}^{2}(\underline{1,2})\right)$ tais que para $i=1,2$

$$
\partial x_{i}=\phi\left(\beta_{i}^{Q}\right)-\beta_{i}^{\prime}
$$

Temos então que definindo

$$
z:=a^{\prime}\left(1 ; x_{2}\right)+x_{1}\left(1 ; 1, a^{\prime}\right)-\left(x_{2}\left(1 ; a^{\prime}, 1\right)+a^{\prime}\left(1 ; x_{1}\right)\right)((21) ;(12))
$$

obtemos que

$$
\partial z=\phi\left(\eta^{Q}\right)-\eta^{\prime}
$$

Aplicando então $\phi$ na equação $(*)$ obtemos

$$
\left(\eta^{\prime}+\partial z\right)(I d+((21) ;(12)))=\lambda a^{\prime}\left(l_{c}^{\prime} ; m_{o}^{\prime}\right)+\partial \phi\left(\gamma^{Q}\right)
$$

que pela proposição A.1 nos dá

$$
\eta^{\prime}(I d+((21) ;(12)))=\lambda\left(a^{\prime}\left(l_{c}^{\prime} ; m_{o}^{\prime}\right)\right)+\partial z^{\prime}=a^{\prime}\left(l_{c}^{\prime} ; m_{o}^{\prime}\right)+\partial \gamma^{\prime}
$$

A classe de homologia $\left[a^{\prime}\left(l_{c}^{\prime} ; m_{o}^{\prime}\right)\right]$ gera $H_{1}\left(\mathcal{S} C_{\text {Vor }}^{2} \underline{(2,2)}\right), \operatorname{logo} \lambda=1$.

Por razões de grau temos que $\psi\left(\beta_{1}^{Q}\right)=\psi\left(\beta_{2}^{Q}\right)=0$, e portanto $\psi\left(\eta^{Q}\right)=0$. Analogamente $\psi\left(\gamma^{Q}\right)=0$. Por $\psi$ ser um quasi-isomorfiso existem $\lambda_{m_{o}}, \lambda_{a}, \lambda_{l_{c}} \in \mathbf{k}^{*}$ tais que $\psi\left(x^{Q}\right)=\lambda_{x}[x]$, $\forall x \in\left\{m_{o}, a, l_{c}\right\}$. Aplicando $\psi$ em (1) temos então que

$$
\lambda \lambda_{a} \lambda_{l_{c}} \lambda_{m_{o}}\left[a\left(\left[\left[l_{c}\right] ;\left[m_{o}\right]\right)\right]=0\right.
$$

o que implica que $\lambda=0$, uma contradição. 


\section{Referências Bibliográficas}

[AS94] Axelrod, Scott; Singer, Isadore M. Chern-Simons perturbation theory. II., J. Differ. Geom. 39, no. 1 (1994): 173-213.

[BCR87] Bochnak, Jacek; Coste, Michel; Roy, Marie-Françoise. Géometrie algébrique réelle, Ergebnisse der Mathematik und ihrer Grenzgebiete, 3. Folge, vol. 12, Springer, Berlin, (1987).

[BE74] Barratt, M. G.; Eccles, Peter J. $\Gamma^{+}$-Structures-I: A free group functor for stable homotopy theory. Topology 13.1 (1974): 25-45.

[BF78] Bousfield, Aldridge; Friedlander, Eric. Homotopy theory of $\Gamma$-spaces, spectra, and bisimplicial sets. Springer Lecture Notes in Math., 658 (1978): 80-130.

[BM03] Berger, Clemens; Moerdijk, Ieke. Axiomatic homotopy theory for operads. Commentarii Mathematici Helvetici 78.4 (2003): 805-831.

[BM06] Berger, Clemens; Moerdijk, Ieke. The Boardman-Vogt resolution of operads in monoidal model categories. Topology 45.5 (2006): 807-849.

[BM07] Berger, Clemens; Moerdijk, Ieke. Resolution of coloured operads and rectification of homotopy algebras. Contemporary Mathematics 431 (2007): 31-58.

[BM11] Berger, Clemens; Moerdijk, Ieke. On an extension of the notion of Reedy category. Mathematische Zeitschrift 269.3-4 (2011): 977-1004.

[BV68] Boardman, John Michael; Vogt, Rainer M. Homotopy-everything H-spaces. Bulletin of the American mathematical society 74.6 (1968): 1117-1122.

[BV73] Boardman, John Michael; Vogt, Rainer M. Homotopy invariant algebraic structures on topological spaces, Lect. Notes Math. 347 (1973).

[BW85] Barr, Michael; Wells, Charles. Toposes, triples and theories. Springer-Verlag, 278 (1985).

[CLM76] Cohen, F. Ronald; Lada, T. Joseph; May, J. Peter. The homology of iterated loop spaces. Lecture Notes in Mathematics, 533 (1976).

[Du16] Ducoulombier, Julien. Swiss-cheese action on the totalization of action-operads. Algebraic \& Geometric Topology 16.3 (2016): 1683-1726. 
120 REFERÊNCIAS BIBLIOGRÁFICAS

[Du17] Ducoulombier, Julien. "From maps between coloured operads to Swiss-Cheese algebras.”arXiv preprint arXiv:1603.07162v3 (2017).

[EM09] Elmendorf, Anthony D.; Mandell, Michael A. Permutative categories, multicategories and algebraic K-theory. Algebraic \& Geometric Topology 9.4 (2009): 2391-2441.

[ESt67] Steenrod, N. E. A convenient category of topological spaces. Michigan Math. J., 14 no. 2 (1967): 133-152.

[FP90] Fritsch, Rudolf; Piccinini, Renzo A. Cellular structures in topology. Cambridge University Press, 15 (1990).

[Fr13] Frankhuizen, Robin. The Recognition Principle for Grouplike $C_{n}$-algebras. MS thesis. 2013.

[GJ95] Getzler, Ezra; Jones, John DS. Operads, homotopy algebra and iterated integrals for double loop spaces. arXiv preprint hep-th/9403055 (1994).

[GJ09] Goerss, Paul G.; Jardine, John F. Simplicial homotopy theory. Springer Science \& Business Media, (2009).

[Hi09] Hirschhorn, Philip S. Model categories and their localizations. American Mathematical Soc., 99 (2009).

[Hi15] Hirschhorn, Philip S. "The Quillen model category of topological spaces."arXiv preprint arXiv:1508.01942 (2015).

[HLS16] Hoefel, Eduardo; Livernet, Muriel; Stasheff, Jim. $A_{\infty}$-actions and recognition of relative loop spaces. Topology and its Applications 206 (2016): 126-147.

[HL12] Hoefel, Eduardo; Livernet, Muriel. Open-closed homotopy algebras and strong homotopy Leibniz pairs through Koszul operad theory. Letters in Mathematical Physics 101.2 (2012): 195-222.

[HoE07] Hoefel, Eduardo. OCHA and the Swiss-cheese operad. arXiv preprint arXiv:0710.3546 (2007).

[HoE11] Hoefel, Eduardo. Explicit Homotopy Equivalences Between Some Operads. arXiv preprint arXiv:1110.3116 (2011).

[HoM98] Hovey, Mark. Monoidal model categories. arXiv preprint math/9803002 (1998).

[HoM07] Hovey, Mark. Model categories. American Mathematical Soc., 63 (2007).

[Id17] Idrissi, Najib. Swiss-cheese operad and Drinfeld center. Israel Journal of Mathematics 221.2 (2017): 941-972. 
[Ko99] Kontsevich, Maxim. Operads and motives in deformation quantization. Letters in Mathematical Physics 48.1 (1999): 35-72.

[KS06] Kajiura, Hiroshige; Stasheff, Jim. Homotopy algebras inspired by classical open-closed string field theory. Communications in mathematical physics 263.3 (2006): 553-581.

[Li58] Lima, Elon Lages. Duality and Postnikov invariants. Tese, University of Chicago, Chicago (1958).

[Li15] Livernet, Muriel. Non-formality of the Swiss-cheese operad. Journal of Topology 8.4 (2015): 1156-1166.

[Lo15] Loregian, Fosco. This is the (co)end, my only (co)friend. arXiv preprint arXiv:1501.02503 (2015).

[Lu09] Lurie, Jacob. Higher Topos Theory. Princeton University Press AM-170 (2009).

[LV14] Lambrechts, Pascal; Volić, Ismar. Formality of the little N-disks operad. American Mathematical Society, Vol. 230. No. 1079 (2014).

[Ma69] May, J. Peter. Categories of spectra and infinite loop spaces. Category Theory, Homology Theory and Their Applications III. Springer, Berlin, Heidelberg, 448-479 (1969).

[Ma72] May, J. Peter. The geometry of iterated loop spaces, Lecture Notes in Mathematics, 271 (1972).

[Ma74] May, J. Peter. $E_{\infty}$-spaces, group completions and permutative categories. New Developments in Topology, London Math. Soc. Lecture Note Series, 11 (1974).

[Ma83] May, J. Peter. The dual Whitehead theorems. London Math. Soc. Lecture Note Series 86 (1983): 46-54.

[Ma90] May, J. Peter. Weak equivalences and quasifibrations. Lecture Notes in Mathematics, 1425 (1990): 91-101.

[Ma99] May, J. Peter. A concise course in algebraic topology. University of Chicago press, (1999).

[Ma09] May, J. Peter. What precisely are $E_{\infty}$ ring spaces and $E_{\infty}$ ring spectra. New topological contexts for Galois theory and algebraic geometry (BIRS 2008) 16 (2009): 215-282.

[McL13] Mac Lane, Saunders. Categories for the working mathematician. Springer Science \& Business Media, 5 (2013).

[MDS76] McDuff, Dusa; Segal, Graeme. Homology fibrations and the "group-completion" theorem. Inventiones mathematicae, 31.3 (1976): 279-284.

[Mi56] Milnor, John. Construction of universal bundles, I,II. Annals of Mathematics (1956). 
122 REFERÊNCIAS BIBLIOGRÁFICAS

[MMSS01] Mandell, M. A.; May, J. P.; Schwede, S.; Shipley, B. Model categories of diagram spectra. Proceedings of the London Mathematical Society, 82(2), (2001) 441-512.

[Qu67] Quillen, Daniel G. Homotopical algebra. Lecture Notes in Mathematics 43 (1967).

[Qu15] Quesney, Alexandre. Swiss Cheese type operads and models for relative loop spaces. arXiv preprint arXiv:1511.05826 (2015).

[Ri09] Riehl, Emily. "A concise definition of a model category."Preprint, available at http://www. math.jhu.edu/ eriehl/modelcat.pdf (2009).

[Sa01] Salvatore, Paolo. Configuration spaces with summable labels. Cohomological methods in homotopy theory. Birkhäuser, Basel, (2001). 375-395.

[Sp01] Spitzweck, Markus. Operads, algebras and modules in general model categories. arXiv preprint math/0101102 (2001).

[StA72] Strøm, Arne. The homotopy category is a homotopy category. Archiv der Mathematik 23.1 (1972): 435-441.

[StJ63] Stasheff, James D. Homotopy associativity of H-spaces I,II. Trans. Amer. Math. Soc. 108 (1963), 275-292.

[StN09] Strickland, Neil P. The category of CGWH spaces. preprint, 12 (2009).

[Sc97] Schwede, Stefan. Spectra in model categories and applications to the algebraic cotangent complex. Journal of Pure and Applied Algebra, 120 (1997): 77-104.

[Sw75] Switzer, Robert. Algebraic Topology - Homotopy and Homology, Die Grundlehren der Mathematischen Wissenschaften in Einzeldarstellungen, Springer-Verlag, New York, N. Y. 212 (1975).

[Ta03] Tamarkin, Dmitry E. Formality of chain operad of little discs. Letters in Mathematical Physics 66.1 (2003): 65-72.

[Vo99] Voronov, Alexander A. The Swiss-cheese operad. Contemporary Mathematics 239 (1999): 365-374.

[Wi17] Willwacher, Thomas. (Non-) formality of the extended Swiss Cheese operads. arXiv preprint arXiv:1706.02945 (2017).

[Zw98] Zwiebach, Barton. Oriented open-closed string theory revisited. Annals of Physics 267.2 (1998): 193-248. 